\title{
Recommandations
}

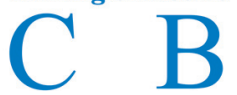

\section{Gestion péri-opératoire des patients traités par antithrombotiques en chirurgie orale. Argumentaire}

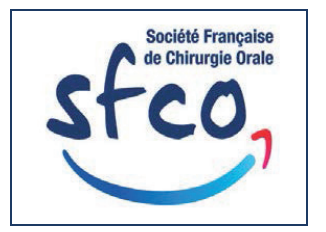

En collaboration avec:
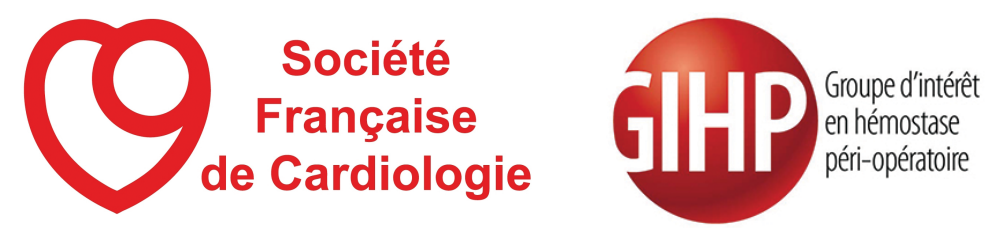

\section{Groupe de travail}

Pr Jean-Christophe FRICAIN (Chirurgie orale, Bordeaux) Président

DrCédric MAUPRIVEZ (Chirurgie orale, Paris) Rapporteur

Dr Pierre GANGLOFF (Chirurgie orale, Metz-Thionville)

Dr Sylvie BOISRAME (Chirurgie orale, Brest)

Dr Patrick LIMBOUR (Chirurgie orale, Rennes)

PrPierre ALBALADEJO (Anesthésie-réanimation, Grenoble)

Dr Annick ANKRI (Hématologie, Paris)

Dr Fabrice CAMPANA (Chirurgie orale, Marseille)

Dr Sarah COUSTY (Chirurgie orale, Toulouse)

Pr Vianney DESCROIX (Pharmacologie, Paris)

Mr Antoine GOBILLARD (Association Malades)

Pr Bernard IUNG (Cardiologie, Paris)

PrIsabelle MAHE(MédecineInterne, Cardiologie, Colombes)

PrJean-Paul MENINGAUD (Chirurgie maxillo-faciale, Créteil)

Dr Jean-Jacques MONSUEZ (Cardiologie, Sevran)

PrEricPAUTAS(MédecineInterne, Gériatrie, Ivry-sur-Seine)

Pr Jacky SAMSON (Stomatologie, Genève)

\section{Groupe de lecture}

Dr Alp ALANTAR (Chirurgie orale, Nanterre)

Dr Ygal ATTAL (Médecine générale, Chambéry)

Dr Anne-Gaelle BODARD (Chirurgie orale, Lyon)

Dr Vanessa BAAROUN (Chirurgie orale, Paris)

Dr Jean-Hugues CATHERINE (Chirurgie orale, Marseille)

Dr Rémi CURIEN (Chirurgie orale, Maxéville)
Dr Christophe DESCHAUMES (Chirurgie orale, Clermont-Ferrand) Dr Émilie DOCHE (Neurologie, Unité Neurovasculaire, Marseille) Dr Célia DUMAS (Médecine générale, Toulouse)

Dr Anne-Laure EJEIL (Odontologie, Paris)

Dr Philippe GUIBELLINO (Médecine d'urgence, Marseille)

Dr Michel GUYOT (Chirurgie orale, Saint-Herblain)

Dr Hilal HAFIAN (Chirurgie orale, Reims)

Dr Emannuelle HERLEM (Médecine générale, Pharmacovigilance, Reims)

Dr Philippe JAUFFRION (Cardiologie, Villejuif)

Dr Nicolas JOVENIN (Cancérologie, Saint-Dizier)

Pr Karine LACUT (Thérapeutique, Brest)

Dr Patrick LARRAS (Chirurgie orale, Dijon)

Dr Florian LAURENT (Odontologie, Igny)

Pr Benoît LEFEVRE (Chirurgie orale, Reims)

Dr Hubert LE HETET (Anesthésie-réanimation, Cesson-Sévigné)

Pr Philippe LESCLOUS (Chirurgie orale, Nantes)

Dr Raphaël LOPEZ (Chirurgie maxillo-faciale, Toulouse)

Pr Louis MAMAN (Chirurgie orale, Ivry-sur-seine)

Dr Jean-Pierre MARIOTTINI (Odontologie, Nice)

Dr Hervé MOIZAN (Chirurgie orale, Rouen)

Pr Jean-Louis MONTASTRUC (Pharmacologie, Toulouse)

Dr Laurent NAWROCHI (Chirurgie orale, Lille)

Dr Laurence NOËL-FURON (Chirurgie orale, Rethel)

Dr William OHANA (Odontologie, Strasbourg)

Pr Guillaume PENEL (Chirurgie orale, Lille)

Dr Omar RAZOUK (Chirurgie orale, Paris)

Dr Marouane TALBI (Chirurgie maxillo-faciale, Metz-Thionville) Dr Nicolas WEISS (Neurologie, Unité de Réanimation neurologique, Paris) 


\section{Abréviations}

\begin{tabular}{|c|c|c|c|}
\hline AAP & Agent(s) anti-plaquettaire(s) & HTA & Hypertension artérielle \\
\hline ACFA & Arythmie cardiaque par fibrillation auriculaire & ICP & Intervention coronaire percutanée \\
\hline ADP & Adénosine diphosphate & IDM & Infarctus du myocarde \\
\hline AINS & Anti-inflammatoires non stéroïdiens & IMC & Indice de masse corporelle \\
\hline AIT & Accident(s) ischémique(s) transitoire(s) & INR & International normalized ratio \\
\hline AMM & Autorisation de mise sur le marché & IV & Intraveineuse \\
\hline AMPc & Adénosine monophosphate cyclique & MDS & Medicament(s) dérivé(s) du sang \\
\hline \multirow[t]{2}{*}{ Anaes } & \multirow{2}{*}{$\begin{array}{l}\text { Agence nationale d'accréditation et d'évaluation } \\
\text { en santé }\end{array}$} & MTEV & Maladie thrombo-embolique veineuse \\
\hline & & NABM & Nomenclature des actes en biologie médicale \\
\hline $\mathrm{AOD}$ & Anticoagulant(s) oral (oraux) direct(s) & NSTEMI & Infarctus du myocarde sans élévation du segment ST \\
\hline AOMI & Artériopathie oblitérante des membres inférieurs & NP & Numération plaquettaire \\
\hline ASA & Aspirine & PMSI & Programme de médicalisation des systèmes \\
\hline AVC & Accident(s) vasculaire(s) cérébral (aux) & & d'information \\
\hline AVK & Antagoniste(s) de la vitamine $\mathrm{K}$ ou antivitamine(s) $\mathrm{K}$ & PTH & Prothèse totale de hanche \\
\hline BNAI & Bloc du nerf alvéolaire inférieur & PTG & Prothèse totale de genou \\
\hline CCP & Concentrés de complexe prothrombinique & SC & Sous-cutanée \\
\hline CIVD & Coagulation intra-vasculaire disséminée & SCA & Syndrome(s) coronarien(s) aigü(s) \\
\hline Cmin & Concentration minimale ou résiduelle & STEMI & Infarctus du myocarde avec élévation du segment ST \\
\hline $\operatorname{cox}-1$ & Cyclo-oxygénase 1 & TCA & Temps de céphaline activée \\
\hline DMS & Dispositif médical stérile & $\mathrm{t}-\mathrm{PA}$ & tissue Plasminogen Activator \\
\hline EP & Embolie(s) pulmonaire(s) & $\mathrm{TIH}$ & Thrombopénie induite par l’héparine \\
\hline ES & Embolie(s) systémique(s) & TQ & Temps de Quick \\
\hline GEHT & Groupe d'étude sur l'hémostase et la thrombose & TS & Temps de saignement \\
\hline GIHP & Groupe d'intérêt sur l'hémostase et la thrombose & TVP & Thrombose(s) veineuse(s) profonde(s) \\
\hline HAS & Haute autorité de santé & TVS & Thrombose(s) veineuse(s) superficielle(s) \\
\hline HBPM & Héparine(s) de bas poids moléculaire & TXA2 & Thromboxane A2 \\
\hline
\end{tabular}




\section{Sommaire}

\section{METHODOLOGIE}

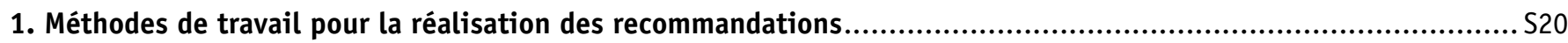

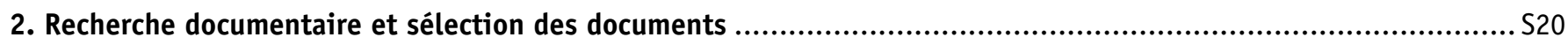

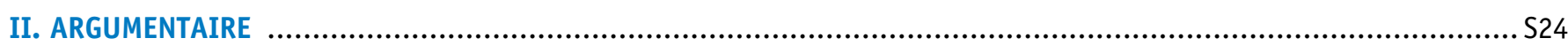

1. Gestion périopératoire des patients traités par antithrombotiques en chirurgie orale (Généralités) ................... S24

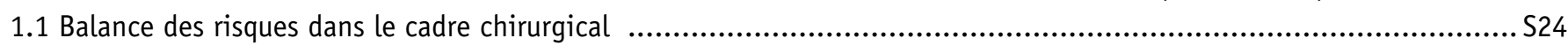

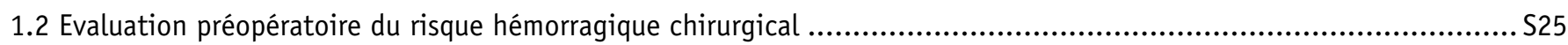

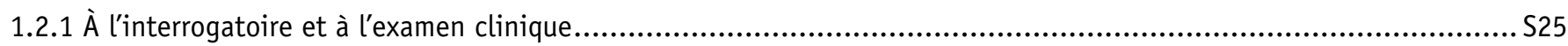

1.2.1.1 Trouble(s) de l'hémostase lié(s) à une pathologie associée ......................................................................S25

1.2.1.2 Compliance du patient vis-à-vis de son traitement antithrombotique ............................................................. S25

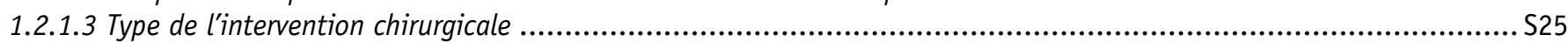

1.2.1.4 Facteurs de risque hémorragique suceptibles de majorer le risque hémorragique chirurgical ...................................S26

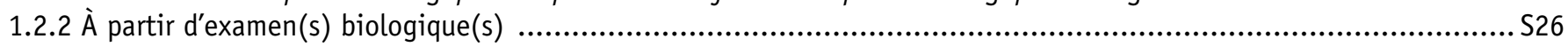

1.3 Choix de la structure de prise en charge : en ville ou en milieu hospitalier ? .................................................... 227

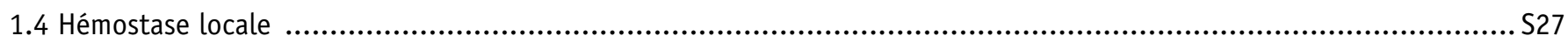

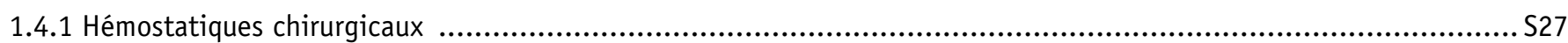

1.4.1.1 Hémostatiques chirurgicaux sans action spécifique sur la cascade d'évènements survenant au cours de l'hémostase .... S27

1.4.1.2 Hémostatiques chirurgicaux avec action spécifique sur la cascade d'évènements survenant au cours de l'hémostase ... S27

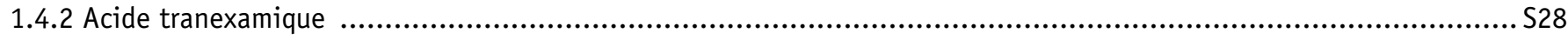

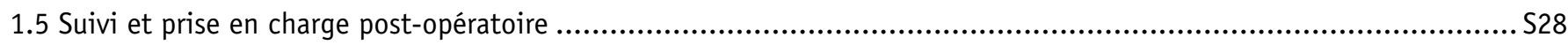

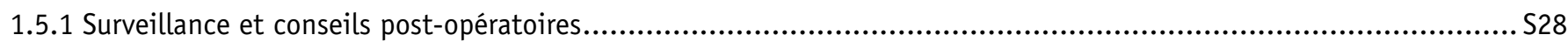

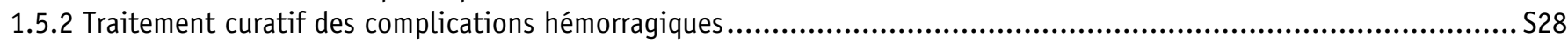

2. Spécificités de la prise en charge des patients traités par agent antiplaquettaires ........................................ S29

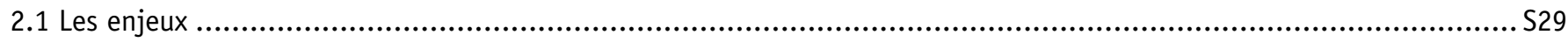

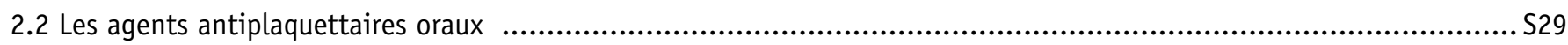

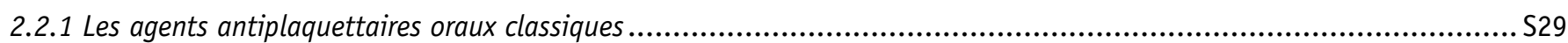

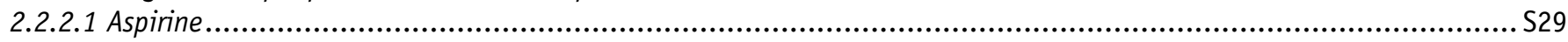

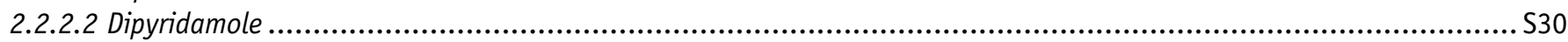

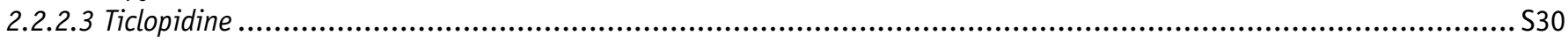

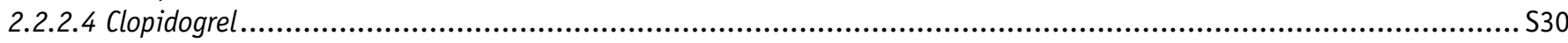

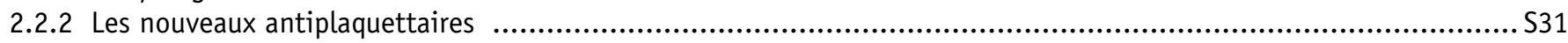

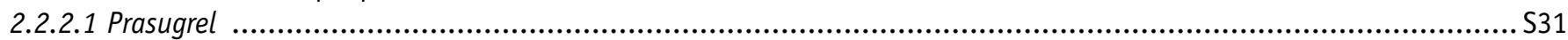

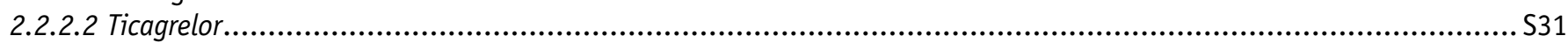

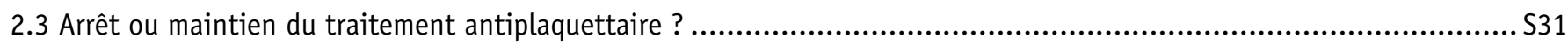

2.4 Quel test biologique doit-on utiliser pour évaluer le risque hémorragique chirurgical ? .......................................S33

2.5 Quelle hémostase locale doit-on réaliser pour contrôler le risque hémorragique chirurgical ? .................................S33

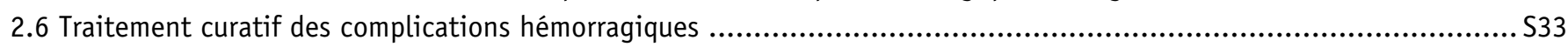

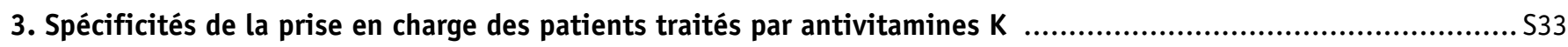

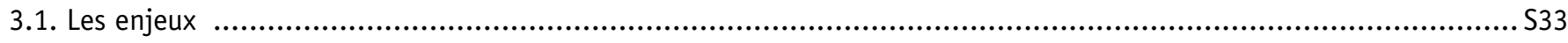

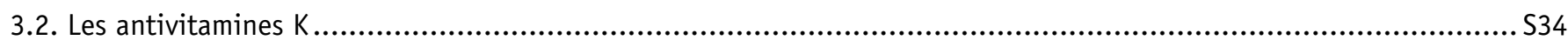

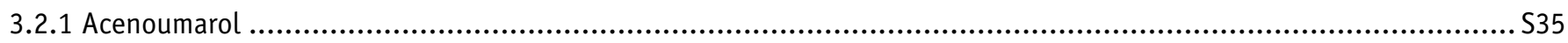

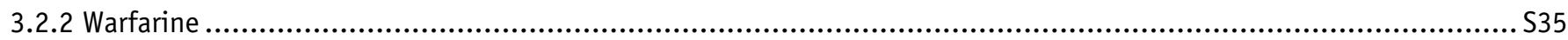

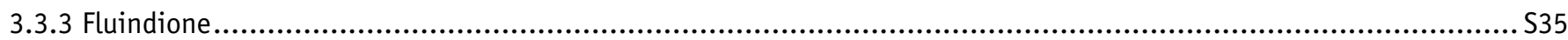

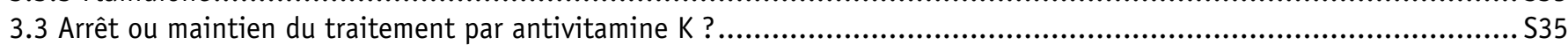

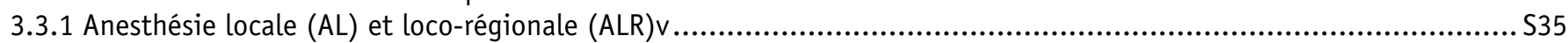




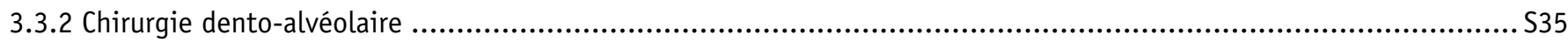

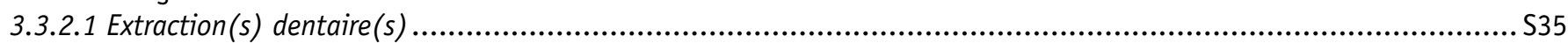

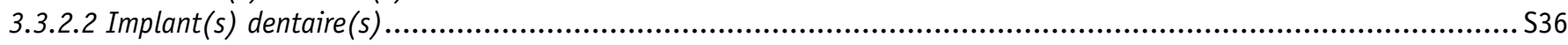

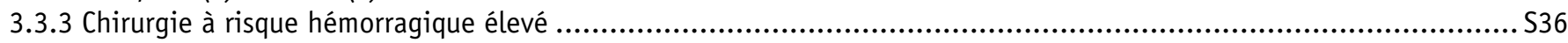

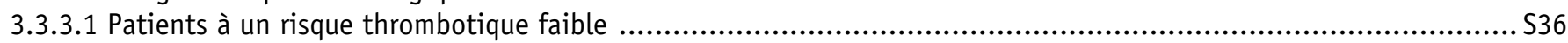

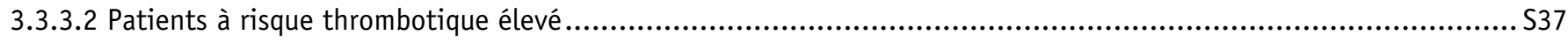

3.4 Quel test biologique doit-on utiliser pour évaluer le risque hémorragique chirurgical ? .......................................S37

3.5 Quelle hémostase locale doit-on réaliser pour contrôler le risque hémorragique chirurgical ? ................................S38

3.6 Quelles sont les prescriptions médicamenteuses potentialisatrices du risque hémorragique ? ................................ S39

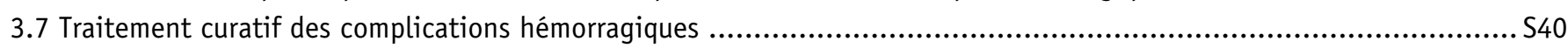

4. Spécificités de la prise en charge des patients traités par anticoagulants oraux directs ............................... S40

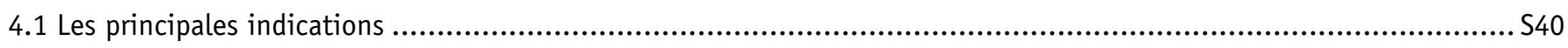

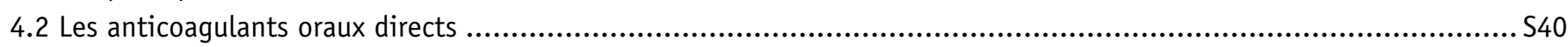

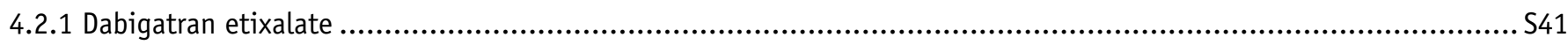

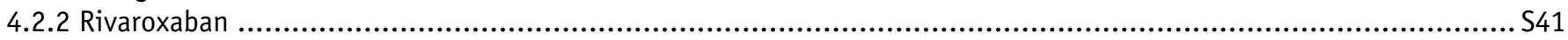

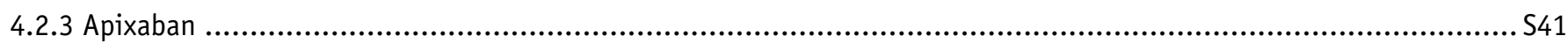

4.3 Arrêt ou maintien du traitement par anticoagulant oral direct ? ............................................................ S42

4.4 Quel test biologique doit-on utiliser pour évaluer le risque hémorragique chirurgical ?....................................... S43

4.5 Quelle hémostase locale peut-on réaliser pour contrôler le risque hémorragique chirurgical ?................................ S43

4.6 Quelles sont les prescriptions médicamenteuses potentialisatrices du risque hémorragique ? ............................... S43

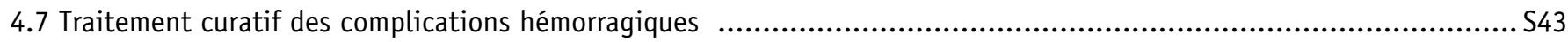

5. Spécificités de la prise en charge des patients traités par anticoagulants injectables .................................... S44

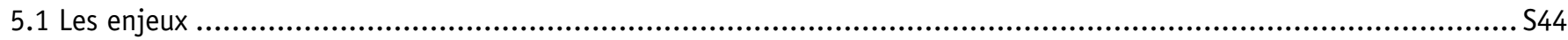

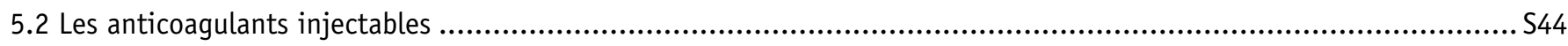

5.2.1 Les héparines standards non fractionnées ................................................................................. S44

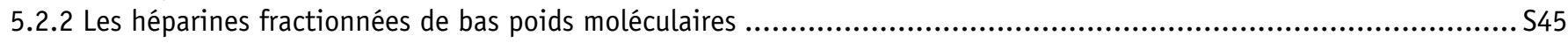

5.3 Arrêt ou maintien du traitement par héparine ? ........................................................................ S45

5.4 Quel test biologique doit-on utiliser pour évaluer le risque hémorragique chirurgical ? ........................................ S46

5.5 Quelle hémostase locale réaliser pour contrôler le risque hémorragique chirurgical ? ........................................S46

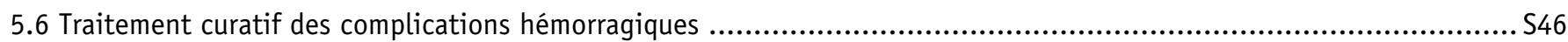

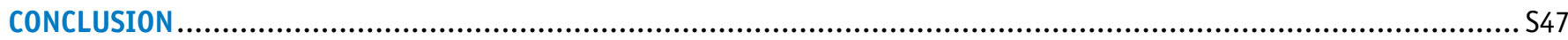

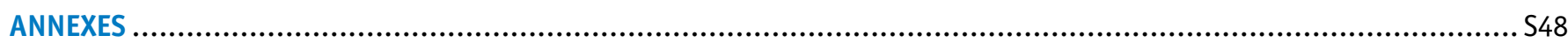

Annexe 1. Antithtrombotiques actuellement commercialisés en France en 2015 ................................................. S48

Annexe 2. Stratification du risque hémorragique en fonction du type de chirurgie et mesures préventives ..................... S49

Annexe 3. Âge et co-morbidités associés à une augmentation du risque d'évènement hémorragique .............................. S49

Annexe 4. Evaluation du risque de saignement et du risque thromboembolique postopératoires en fonction de la stratégie thérapeutique après chirurgie dentoalvéolaire .............................................................. 550

Annexe 5. Patients sous agents antiplaquettaires (aspirine, clopidogrel, ticlopidine) et chirurgie dento-alvéolaire (Études cliniques)

Annexe 6. Patients sous bithérapie antiplaquettaire (aspirine + clopidogrel) et chirurgie dento-alvéolaire (Études cliniques)

Annexe 7. Patients sous AVK (Acénocoumarol, Coumarine, Fluindione, Warfarine) et chirurgie dento-alvéolaire (Études cliniques)

Annexe 8. Patients sous bithérapie antiplaquettaire + AVK et chirurgie dento-alvéolaire (Études cliniques) 
Annexe 9. Protocole d'arrêt et de reprise d'un AVK sans relais héparinique pour une chirurgie

à risque hémorragique élevé chez un patient à risque thrombotique faible

Annexe 10. Protocole d'arrêt et de reprise d'un AVK avec un relais héparinique pour une chirurgie

à risque hémorragique élevé chez un patient à risque thrombotique élevé.

Annexe 11. Principaux paramètres pharmacocinétique des AOD

Annexe 12. Protocole d'arrêt et de reprise d'un AOD sans relais héparinique pour une chirurgie

à risque hémorragique élevé....

Annexe 13. Patients sous héparines (HBPM, HNF) et chirurgie dento-alvéolaire (Études cliniques)

Annexe 14. Algorithme systématique de prise en charge des patients sous antiplaquettaires (AAP)

Annexe 15. Algorithme systématique de prise en charge des patients sous antivitamine K (AVK).....

Annexe 16. Algorithme systémique de prise en charge des patients sous anticoagulants oraux directs (AOD) S70

Annexe 17. Algorithme systématique de prise en charge des patients sous héparines en chirurgie orale S71 


\section{MÉTHODOLOGIE}

\section{Méthode de travail pour la réalisation des recommandations}

Ces recommandations professionnelles ont été élaborées selon la méthode des recommandations pour la pratique clinique, publiée par l'Agence nationale d'accréditation et d'évaluation en santé (Anaes) en janvier 2000 (www.has-sante.fr).

La Société Française de Chirurgie Orale (SfCo), promoteur du projet, a nommé un président qui a constitué un groupe de travail et a coordonné l'ensemble des travaux. Le groupe de travail était composé de professionnels multidisciplinaires, ayant un mode d'exercice public ou privé, d'origine géographique variée, et membres de la Société Française de Cardiologie (Sfc) et de la Société Française d'Anesthésie et Réanimation (Sfar). Un rapporteur a été désigné afin de rédiger les recommandations (texte court et argumentaire), au terme d'une analyse de la littérature scientifique et d'une synthèse de l'avis de l'ensemble des membres participant au groupe de travail.

Un groupe de lecture, composé selon les mêmes critères que le groupe de travail, a été consulté par courrier et a donné un avis sur le fond et la forme des recommandations. Les commentaires du groupe de lecture ont été analysés par le groupe de travail et pris en compte chaque fois que possible dans la rédaction des recommandations.

\section{Recherches documentaires et selection des documents}

Une recherche documentaire approfondie a été effectuée par interrogation des bases de données bibliographiques Medline ${ }^{\circledR}$ et Cochrane ${ }^{\circledR}$.

Les limites communes à l'ensemble des investigations effectuées sur ces bases de données automatisées ont été les suivantes :

- seuls les articles rédigés en anglais et en français ont été sélectionnés ;

- la période de recherche définie allait de 1985 à 2014. (Cette interrogation a été réalisée en janvier 2014, puis une veille documentaire s'est poursuivie jusqu'en janvier 2015) ;

- seuls les essais cliniques, les méta-analyses, les revues systématiques, les recommandations pour la pratique clinique, les conférences de consensus, les publications institutionnelles et les articles de décision médicale ont été analysés.
Les mots clés utilisés étaient soit des termes issus d'un thésaurus (descripteurs du MeSH pour Medline), soit des termes de titre ou de résumé. Les mots clefs sélectionnés étaient:

« Antithrombotic », « Antiplatelet », « Aspirin », « Dipyridamol », « Ticlopidin », « Clopidogrel », « Prasugrel », « Ticagrelor », « Anticoagulant », « Antivitamin K », « Warfarin », «Acenocoumarol », « Fluindione », « New oral anticoagulants », « Novel anticoagulants », « Direct oral anticoagulants », « Direct thrombin inhibitor », « Factor Xa inhibitors », « Dabigatran », « Rivaroxaban », « Apixaban », « Injectables anticoagulants » « Heparins », « Low molecular weight heparins », « Enoxaparin », « Nadroparin », « Daltéparin », « Tinzaparin », « Fondaripanux », « Hemorrhage », « Bleeding », « Hemostasis », « Hemostatic agent », « Topic hemostatic », « Topic hemostatic agent », « Surgery hemostatic », « Tooth extraction », «Dento-alveolar surgery », « Dento-alveolar surgery », « Dental implant ».

Les mots clés ont ensuite été combinés en autant d'étapes que nécessaire à l'aide des opérateurs boléens « ET », « OU ».

Le tableau 1 présente le détail de la stratégie de recherche sur Medline ainsi que les résultats en matière de nombre total de références obtenues, ainsi que celles retenues pour répondre aux questions posées.

Les articles identifiés ont été analysés selon les principes de lecture critique de la littérature à l'aide de grilles de lecture. Seuls les articles pertinents ont été retenus et il a été affecté pour chaque article sélectionné un niveau de preuve scientifique.

Lors de cette recherche bibliographique, il a été aussitôt mis en évidence le faible nombre d'études et/ou de faible niveau de preuve concernant les $A 0 D$, les héparines et la chirurgie pré-implantaire.

C'est pourquoi, un deuxième type de recherche a été effectué. Les sites internets des sociétés savantes nationales (Groupe Étude de l'Hémostase et de la Thrombose (GEHT) de la Société d'Hématologie, Société Française de Chirurgie Orale, Société Française d'Anesthésie et de Réanimation) internationales (American Dental Association, American College of Chest Physicians, British Dental Association, Bristish Committee for Standards in Haematology, International Society of Thrombosis and Haemostasis, World Workshop in Oral Medecine), les sites internets d'agences institutionnelles (HAS, Afssaps, Ansm) ainsi que des ouvrages pouvant avoir un rapport avec le thème ont été consultés.

La liste des sites consultés ainsi que les documents sélectionnés figurent dans le tableau 2.

Selon le niveau de preuve des études, des recommandations sont proposées et gradée (côté de $\mathrm{A}$ à C) selon l'échelle proposée par la HAS (tableau $\underline{3}$ ). En l'absence d'études, les recommandations sont fondées sur un accord professionnel $(\mathrm{AE})$. 
Tableau I. Stratégie d'interrogation documentaire dans la base documentaire Medline.

\begin{tabular}{|c|c|c|c|}
\hline Sujet & \multicolumn{3}{|c|}{ Risque hémorragique en chirurgie orale pour les patients sous antiplaquettaires } \\
\hline & Termes utilisés & Nb de réf. & Nombre de références retenues \\
\hline Etape 1 & $\begin{array}{l}\text { " Antiplatelet » OU « Aspirin » OU « Dipyridamol » OU } \\
\text { « Ticlopidin » OU « Clopidogrel » OU « Prasugrel » OU } \\
\text { « Ticagrelor » }\end{array}$ & 17381 & \\
\hline Etape 2 & 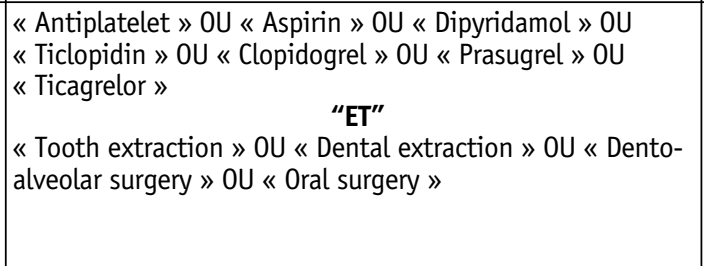 & 28 & $\begin{array}{l}\text { Aframian, } 2007 \text { [1], Ardekian, } 2000 \text { [24], Bajkin, } 2012 \text { [35], Bajkin, } \\
2015[36], \text { Brennan, } 2008 \text { [49], Canigral, 2008 [54] Cardona-Tortajada, } \\
2009 \text { [56], Garnier, 2007 [113], Iwabuchi, 2014 [173], Krishman, 2008 } \\
\text { [185], Lillis, 2011[197], Madan, 2010 [204],] Medeiros, } 2011 \text { [212], } \\
\text { Moritomoto, 2008a [223], Morimoto, 2011 [225], Napenas, 2009 } \\
\text { [227], Olmos-Carrasco, 2015 [234], Park, 2012 [239], Partridge, 2008 } \\
\text { [237], Rai, 2013 [248], van Diermen, 2009 [278], Verma, 2013 [280], } \\
\text { Verma, } 2014 \text { [281] }\end{array}$ \\
\hline Etape 3 & $\begin{array}{l}\text { «Antiplatelet» OU " «Aspirin» OU «Dipyridamol» OU } \\
\text { «Ticlopidin»0U «Clopidogrel»0U «Prasugrel»0U «Ticagrelor» } \\
\text { "ET" } \\
\text { « Dental implant » }\end{array}$ & 3 & Aucune référence retenue \\
\hline Etape 4 & 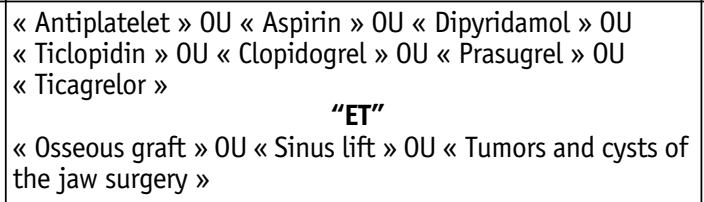 & 3 & Aucune référence retenue \\
\hline Sujet & Risque hémorragique en chirurgie orale pour les patients sous & antivitamines & \\
\hline & Termes utilisés & Nb de réf. & Nombre de références retenues \\
\hline Etape 1 & $\begin{array}{l}\text { «Oral anticoagulant » } 0 \mathrm{U} \text { « Antivitamin } \mathrm{K} » 0 \mathrm{U} \text { « Warfarin » } \\
\mathrm{OU} \text { « Acenocoumarol » } \mathrm{OU} \text { « Fluindione » }\end{array}$ & 16542 & \\
\hline Etape 2 & \begin{tabular}{|l} 
« Oral Anticoagulant » $0 \mathrm{U}$ « Antivitamin $\mathrm{K} » 0 \mathrm{U}$ « Warfarin » \\
OU « Acenocoumarol » $\mathrm{OU}$ « Fluindione » \\
"ET" \\
« Tooth extraction » $0 \mathrm{U}$ « Dental extraction » $\mathrm{OU}$ « Dento- \\
alveolar surgery » $0 \mathrm{U}$ « Oral surgery »
\end{tabular} & 274 & 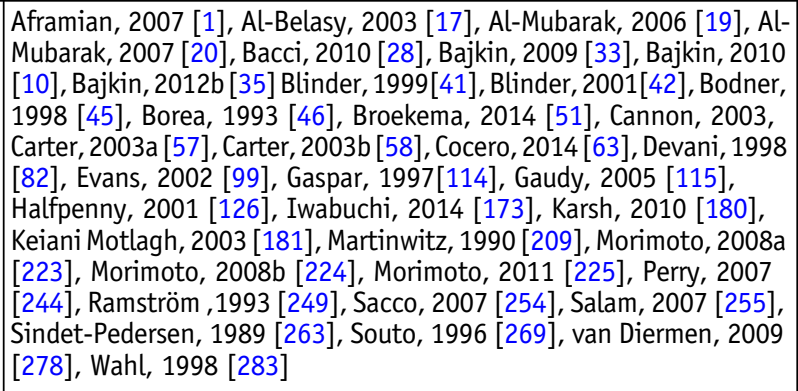 \\
\hline Etape 3 & $\begin{array}{l}\text { «Oral anticoagulant » } 0 \mathrm{U} \text { «Antivitamin } \mathrm{K} » \mathrm{OU} \text { « Warfarin » } \\
\mathrm{OU} \text { « Acenocoumarol » } \mathrm{OU} \text { « Fluindione » } \\
\text { "ET" } \\
\text { « Dental implant » }\end{array}$ & 58 & Bacci, 2011 [29], Madrid et Sanz, 2009 [205] \\
\hline Etape 4 & $\begin{array}{l}\text { «Oral anticoagulant » } \mathrm{OU} \text { « Antivitamin } \mathrm{K} » \mathrm{OU} \text { « Warfarin » } \\
\mathrm{OU} \text { «Acenocoumarol » } \mathrm{UU} \text { « Fluindione » } \\
\text { "ET" } \\
\text { « Osseous graft » } \mathrm{OU} \text { « Sinus lift » } \mathrm{OU} \text { « Tumors and cysts of } \\
\text { the jaw surgery » }\end{array}$ & 12 & Aucune référence retenue \\
\hline
\end{tabular}


Tableau I. Suite.

\begin{tabular}{|c|c|c|c|}
\hline Sujet & \multicolumn{3}{|c|}{ Risque hémorragique en chirurgie orale pour les patients sous bithérapie antiplaquettaire et AVK } \\
\hline Etape 1 & $\begin{array}{l}\text { «Oral anticoagulant » } 0 \mathrm{U} \text { « Antivitamin } \mathrm{K} » 0 \mathrm{U} \text { « Warfarin » } \\
\text { ET } \\
\text { « Antiplatelet » } 0 \mathrm{U} \text { « Aspirin » } 0 \mathrm{U} \text { « Clopidogrel » }\end{array}$ & 877 & \\
\hline Etape 2 & $\begin{array}{l}\text { « Oral anticoagulant » } 0 \mathrm{U} \text { « Antivitamin } \mathrm{K} » 0 \mathrm{U} \text { « Warfarin » } \\
\mathrm{ET} \\
\text { « Antiplatelet » } \mathrm{OU} \text { « Aspirin » } \mathrm{OU} \text { « Clopidogrel » } \\
\text { ET } \\
\text { « Tooth extraction » } 0 \mathrm{U} \text { « Dental extraction » } 0 \mathrm{U} \text { « Dento- } \\
\text { alveolar surgery » } \mathrm{U} \text { 《 Oral surgery » }\end{array}$ & 10 & $\begin{array}{l}\text { Bajkin, 2012b [35], Cardona-Tortadaja, } 2009 \text { [56], Morimoto, 2008a } \\
\text { [223], Morimoto, 2011 [225], Perry, } 2007 \text { [144], Pototski, } 2007 \text { [247], } \\
\text { van Diermen, } 2009 \text { [278] }\end{array}$ \\
\hline Etape 3 & $\begin{array}{l}\text { 《Oral anticoagulant » } 0 \mathrm{U} \text { « Antivitamin } \mathrm{K} » 0 \mathrm{U} \text { « Warfarin » } \\
\text { ET } \\
\text { « Antiplatelet » } \mathrm{OU} \text { « Aspirin » } 0 \mathrm{U} \text { « Clopidogrel » } \\
\text { ET } \\
\text { « Dental implant » }\end{array}$ & 0 & Aucune référence \\
\hline Etape 4 & $\begin{array}{l}\text { «Oral anticoagulant » } 0 \mathrm{U} \text { « Antivitamin } \mathrm{K} » 0 \mathrm{U} \text { « Warfarin » } \\
\mathrm{ET} \\
\text { «Antiplatelet » } \mathrm{OU} \text { « Aspirin » } \mathrm{OU} \text { « Clopidogrel » } \\
\text { ET } \\
\text { « Osseous graft » } \mathrm{OU} \text { « Sinus lift » } \mathrm{OU} \text { « Tumors and cysts of } \\
\text { the jaw surgery » }\end{array}$ & 0 & Aucune référence \\
\hline Sujet & Risque hémorragique en chirurgie orale pour les patients sous & anticoagulan & ts oraux directs \\
\hline & Termes utilisés & Nb de réf. & Nombre de références retenues \\
\hline Etape 1 & $\begin{array}{l}\text { «New oral anticoagulants » } 0 \mathrm{U} \text { « Novel anticoagulants » } 0 \mathrm{U} \\
\text { « Directs oral anticoagulants » } 0 \mathrm{U} \text { « Direct thrombin } \\
\text { inhibitor » } 0 \mathrm{U} \text { « Factor Xa inhibitors » } 0 \mathrm{U} \text { « Dabigatran » } 0 \mathrm{U} \\
\text { « Rivaroxaban » OU « Apixaban » }\end{array}$ & 3142 & \\
\hline Etape 2 & 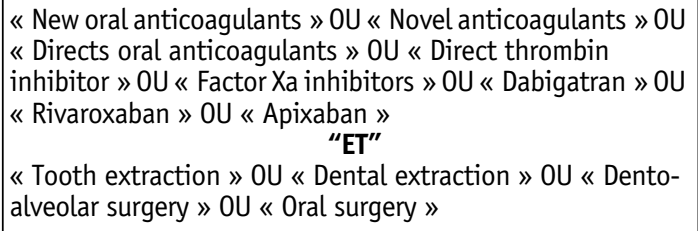 & 12 & $\begin{array}{l}\text { Breik, } 2014 \text { [47], Davis, } 2013 \text { [77], Firriolo, } 2012 \text { [105], Romond, } 2013 \\
\text { [293] }\end{array}$ \\
\hline Etape 3 & $\begin{array}{l}\text { « New oral anticoagulants » } 0 \mathrm{U} \text { « Novel anticoagulants » } \mathrm{OU} \\
\text { «Directs oral anticoagulants } 0 \mathrm{U} \text { « Direct thrombin inhibitor » } \\
\text { OU « Factor Xa inhibitors » } 0 \mathrm{U} \text { « Dabigatran » } 0 \mathrm{U} \\
\text { « Rivaroxaban » OU « Apixaban » } \\
\text { « "Eental implant » }\end{array}$ & 2 & Aucune référence retenue \\
\hline Etape 4 & 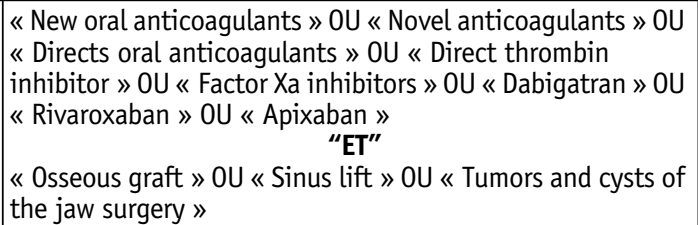 & 0 & Aucune référence retenue \\
\hline
\end{tabular}


Tableau I. Suite.

\begin{tabular}{|c|c|c|c|}
\hline Sujet & \multicolumn{3}{|c|}{ Risque hémorragique en chirurgie orale pour les patients sous anticoagulants injectables (héparines, fondaripanux) } \\
\hline & Termes utilisés & Nb de réf. & Nombre de références retenues \\
\hline Etape 1 & $\begin{array}{l}\text { «Injectables anticoagulants » } \mathrm{OU} \text { « Heparins » } 0 \mathrm{U} \text { « Low } \\
\text { molecular weight heparins » } 0 \mathrm{U} \text { « Enoxaparin » } 0 \mathrm{U} \\
\text { « Nadroparin » } 0 \mathrm{U} \text { « Daltéparin » } 0 \mathrm{U} \text { « Tinzaparin » } 0 \mathrm{U} \\
\text { « Fondaripanux » }\end{array}$ & 13222 & \\
\hline Etape 2 & 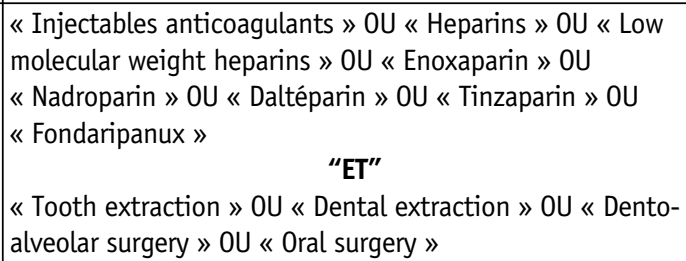 & 8 & $\begin{array}{l}\text { Bajkin, } 2009 \text { [33], Hong, } 2010 \text { [167], Karsh, } 2011 \text { [180], Morimoto, } \\
\text { 2008b [224], Morimoto, } 2012 \text { [226] }\end{array}$ \\
\hline Etape 3 & 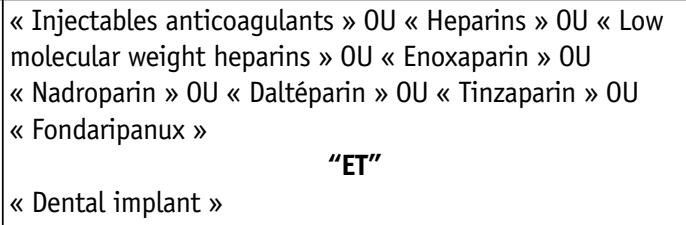 & 0 & Aucune référence \\
\hline Sujet & \multicolumn{3}{|l|}{ Hémorragie et chirurgie orale } \\
\hline & Termes utilisés & Nb de réf. & Nombre de références retenues \\
\hline Etape 1 & « Hemorrhage » 0U « Bleeding » & 56425 & \\
\hline Etape 2 & $\begin{array}{l}\text { « Hemorrhage » OU « Bleeding » } \\
\text { "ET" } \\
\text { « Tooth extraction » OU « Dental extraction » OU « Dento- } \\
\text { alveolar surgery » OU « Oral surgery » }\end{array}$ & 78 & $\begin{array}{l}\text { Czimbireck, } 2014 \text { [72], Lockhart, } 2003 \text { [199], Chee, } 2008 \text { [61], Cosmi, } \\
2009 \text { [70], Iwabucchi, } 2014 \text { [173], Moghadam, } 2002 \text { [218], Pister, } \\
2010 \text { [246] }\end{array}$ \\
\hline Etape 3 & $\begin{array}{l}\text { « Hemorrhage » } 0 \mathrm{U} \text { « Bleeding » } \\
\text { "ET" } \\
\text { « Dental implant » }\end{array}$ & 16 & $\begin{array}{l}\text { Bruggenkate, } 1993 \text { [53], Darriba, } 1997 \text { [75], Givol, 2000 [117], Hong, } \\
2011 \text { [169], Jensen, } 2012 \text { [175], Kalpidis, } 2005 \text { [179], Laboda, } 1990 \\
\text { [186], Masson, } 1190 \text { [211], Mordenfeld, } 1997 \text { [220], Niamtu, } 2001 \\
\text { [230], Panula, } 1999 \text { [235] }\end{array}$ \\
\hline Sujet & Hémostase locale et chirurgie orale en chirurgie orale & & \\
\hline & Termes utilisés & Nb de réf. & Nombre de références retenues \\
\hline Etape 1 & «Antithrombotics» & 34895 & \\
\hline Etape 2 & 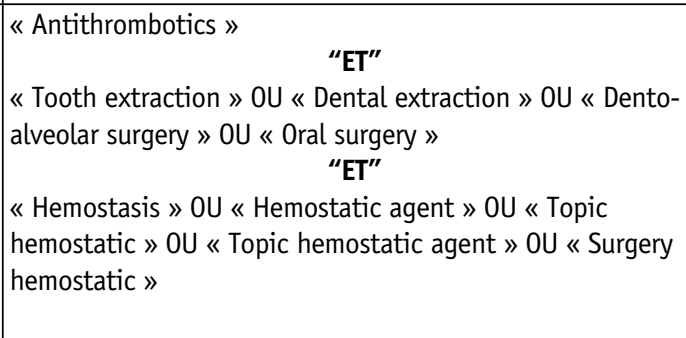 & 116 & $\begin{array}{l}\text { Aframian, } 2007 \text { [1], Al Belasy, } 2003 \text { [17], Al-Mubarak, } 2006 \text { [19], Al- } \\
\text { Mubarak, } 2007 \text { [20], Bacci, } 2010 \text { [28], Bajkin, } 2009 \text { [33] } \\
\text { Bajkin, 2012b [35], Beirne, } 1996 \mid 39], \text { Blinder, } 1999 \text { [41], Borea, } 1993 \\
\text { [46], Brennan, 2008 [49], Broekema, 2013 [51], Carter, 2003a [57], } \\
\text { Carter, 2003b [58], Evans, 2002 [99], Halpenny, } 2001 \text { [126], Kalpidis, } \\
2005[179], \text { Karsh., 2011 [180], Keiani Motlagh, } 2003 \text { [181], Medeiros, } \\
2011 \text { [212], Morimoto, 2008a [223], Patatanian, } 2006 \text { [236], Perry, } \\
2007 \text { [244], Pototski, 2007 [247], Ramström, } 1993 \text { [249], Sindet- } \\
\text { Pedersen., } 1989 \text { [263], Souto., 1996 [269], van Diermen, } 2009 \text { [278] }\end{array}$ \\
\hline
\end{tabular}


Tableau II. Liste des sites des sociétes savantes (internationales et nationales) et les établissements publics placés sous la tutelle du ministère chargé de la santé consultés.

$\left.\begin{array}{|l|c|l|}\hline \multicolumn{1}{|c|}{\text { Sociétés savantes et établissements publics }} & \begin{array}{c}\text { Nombre } \\ \text { de référence }\end{array} & \multicolumn{1}{c|}{\text { Références sélectionnées }} \\ \hline \text { Agence française de sécurité sanitaire des produits de santé (Afssaps) } & 5 & {[2],[3],[4],[6],[7]} \\ \hline \text { Agence nationale de la sécurité du médicament (Ansm) } & 3 & {[9],[10],[11]} \\ \hline \text { American Dental Association (ADA) } & 1 & {[121]} \\ \hline \text { American College of Chest Physicians } & 7 & {[12],[61],[85],[86],[91],[111],[125]} \\ \hline \text { American Heart Association (AHA) } & 1 & {[121]} \\ \hline \text { British Dental Society (BDS) } & 1 & {[244]} \\ \hline \text { Bristish Committee for Standards in Haematology } & 2 & {[32],[207]} \\ \hline \text { International Society of Thrombosis and Haemostasis (ISTH) } & 1 & {[111]} \\ \hline \begin{array}{l}\text { Groupe Etude de l'Hémostase et de la Thrombose (GEHT) de la Société } \\ \text { d'Hématologie }\end{array} & 3 & {[122],[123],[124],[259],[260]} \\ \hline \text { Haute Autorité de Santé (HAS) } & 34 & {[130],[131],[132],[133],[134],[135],[136],[137],[138],[139],[140],} \\ {[141],[142],[143],[144],[145],[146],[147],[148],[149],[150],[151],} \\ {[152],[153],[154],[155],[156],[157],[158],[159],[160],[161],[162],} \\ {[163]}\end{array}\right]$

Tableau III. Grade des recommandations.

\begin{tabular}{|c|l|}
\hline \multicolumn{1}{|c|}{ Grade des recommandations } \\
\hline A & $\begin{array}{l}\text { Preuve scientifique établie } \\
\text { Fondée sur des études de fort niveau de preuve (niveau de preuve 1) : essais comparatifs randomisés de forte puissance et sans biais majeur ou méta- } \\
\text { analyse d'essais comparatifs randomisés, analyse de décision basée sur des études bien menées. }\end{array}$ \\
\hline B & $\begin{array}{l}\text { Présomption scientifique } \\
\text { Fondée sur une présomption scientifique fournie par des études de niveau intermédiaire de preuve (niveau de preuve 2), comme des essais comparatifs } \\
\text { randomisés de faible puissance, des études comparatives non randomisées bien menées, des études de cohorte. }\end{array}$ \\
\hline C & $\begin{array}{l}\text { Faible niveau de preuve } \\
\text { des ée sur des études de moindre niveau de preuve, comme des études cas-témoins (niveau de preuve 3), des études rétrospectives, des séries de cas, }\end{array}$ \\
\hline AE & $\begin{array}{l}\text { En l'absence d'études, les recommandations sont fondées sur un accord entre experts du groupe de travail, après consultation du groupe de lecture. L'absence } \\
\text { de gradation ne signifie pas que les recommandations ne sont pas pertinentes et utiles. Elle doit, en revanche, inciter à engager des études complémentaires }\end{array}$ \\
\hline
\end{tabular}

\section{ARGUMENTAIRE}

\section{Gestion péri-opératoire des patients traités par antithrombotiques en chirurgie orale (Généralités)}

\subsection{Balance des risques dans le cadre chirurgical}

L'interruption d'un traitement antithrombotique efficace et reposant sur une indication reconnue ne peut se concevoir sans une augmentation du risque thrombotique. Pour les agents anti-plaquettaires (AAP), comme pour les antivitamines (AVK), l'analyse de la littérature montre que l'arrêt d'un traitement antithrombotique s'accompagne d'une augmentation significative du nombre d'évènements thrombotiques artériel et/ou veineux durant la période postopératoire $[40,64,65,102$, 283].
Des trois stratégies de gestion des antithrombotiques : le maintien du traitement, l'arrêt complet ou partiel (en cas de bithérapie) du traitement et le recours à un traitement substitutif, l'option thérapeutique à privilégier en première intention est la poursuite (la non-interruption) du traitement antithrombotique [1, 26, 48, 83, 183, 228, 244, 278]. Il est donc impératif pour le praticien d'identifier le risque hémorragique vis-à-vis de l'acte chirurgical envisagé et sa faisabilité sous antithrombotique.

- En chirurgie dento-alvéolaire, le saignement de faible intensité est aisément contrôlable par une hémostase locale. Le surcoût d'événement hémorragique est donc acceptable en cas de maintien du traitement antithrombotique ; la poursuite du traitement antithrombotique permettant par ailleurs de garantir une sécurité antithrombotique optimale durant la période péri-opératoire $[1,26,48,83,183,228$, 244, 278]. 
- En revanche, les procédures chirurgicales à risque hémorragique élevé (ex : greffe osseuse, chirurgie des kystes et tumeurs des maxillaires) ou sans possibilité de contrôler le saignement induit (ex : élévation du sinus), en l'absence de contre-indications médicales (ex : prévention de l'endocardite infectieuse) plaide en faveur de l'arrêt des antithrombotiques. Une consultation avec le médecin prescripteur est dès lors indispensable pour évaluer le risque thrombotique et définir la stratégie thérapeutique à adopter. Dans tous les cas, l'arrêt d'un traitement antithrombotique est de la responsabilité du médecin prescripteur.

La liste des médicaments antithrombotiques actuellement commercialisés en France en 2015, les différentes spécialités et leurs indications sont présentés en annexe 1 .

\subsection{Evaluation préopératoire du risque hémorragique chirurgical}

La seule prise de médicament(s) antithrombotique(s) et de leur éventuel maintien au cours d'une chirurgie ne doit pas occulter tous les autres facteurs susceptibles d'aggraver le risque de saignement péri-opératoire. Ces facteurs de risque sont nombreux et ne doivent en aucun cas être négligés [72, 109, 199].

L'évaluation du risque hémorragique chirurgical repose avant tout sur l'interrogatoire médical et l'examen clinique préopératoire. Celui-ci peut en fonction des données cliniques et/ ou de la molécule antithrombotique être complété par la prescription d'examens biologiques [44].

\subsubsection{Interrogatoire et examen clinique}

Les objectifs de cette évaluation pré-opératoire du risque hémorragique sont multiples. Le praticien doit :

- s'assurer que le patient ne présente pas d'autre anomalie de l'hémostase (qu'elle soit constitutionnelle ou acquise) ou à une co-morbidité (insuffisance hépatique, insuffisance rénale) ;

- s'assurer de la bonne compliance du patient vis-à-vis de son traitement antithrombotique, et que celui-ci ne présente pas de signes cliniques et/ou biologiques d'un éventuel surdosage ;

- classer l'intervention chirurgicale ou l'acte invasif en fonction de la prédictibilité du risque hémorragique qui lui est associé (contrôlable ou non par des mesures simples d'hémostase mécanique, localisation critique ou non) ;

- identifier les facteurs de risque hémorragique (liés au patient, liés à la chirurgie) susceptibles de majorer le risque hémorragique chirurgical.

L'interrogatoire médical et l'examen clinique doivent être structurés. De nombreuses études ont montré qu'un interrogatoire non-structuré est prédictif d'un sur-risque hémorragique chirurgical $[61,70]$.

\subsubsection{Trouble(s) de l'hémostase lié(s) à une pathologie associée}

De nombreux questionnaires avec une sensibilité élevée et une valeur prédictive positive permettent de dépister une éventuelle pathologie de la coagulation associée. Cette situation est rare mais elle ne doit pas être écartée [61].

\subsubsection{Compliance du patient vis-à-vis de son traitement antithrombotique}

Lors de l'interrogatoire, le nom du ou des médicament(s) antithrombotique(s), la dose, le nombre de prise par jour, l'indication du traitement antithrombotique, ainsi que le suivi biologique et la valeur des derniers examens connue (ex : INR, Numération plaquettaire, TCA) doivent être notifiés.

Au moment de l'examen clinique, les signes cliniques évocateurs d'une coagulopathie ou d'un surdosage doivent être recherchés (épistaxis, gingivorragies $>3$ minutes après le brossage, ecchymose cutanée et/ou muqueuse...).

\subsubsection{Type d'intervention chirurgicale}

En chirurgie orale, l'élément majeur d'appréciation du risque hémorragique chirurgical reste la possibilité d'un contrôle du saignement postopératoire par des mesures simples d'hémostase mécanique (pression + sutures). Aussi, il est licite de distinguer en chirurgie orale, les interventions chirurgicales pour lesquelles une hémorragie extériorisée est facilement contrôlable par une hémostase chirurgicale conventionnelle de celles difficilement contrôlables par les moyens usuels. Le deuxième élément important à considérer est la localisation de l'hémorragie potentielle et de ses conséquences sur le pronostic vital ou fonctionnel. En chirurgie orale, les localisations dites " critiques » tels que le plancher buccal, la langue et le sinus maxillaire, sont des régions anatomiques au niveau desquelles une hémorragie peut devenir rapidement incontrôlable en pratique de ville. Les autres éléments discriminants pouvant impacter le risque hémorragique chirurgical sont la nature des tissus opérés (chirurgie osseuse et chirurgie des tissus mous), la technique chirurgicale et le mode opératoire (chirurgie traditionnelle avec des incisions et décollements larges versus chirurgie mini-invasive), l'expérience chirurgicale du praticien, la durée de l'intervention (temps opératoire $>1$ heure ou $\leq 1$ heure), et la modalité de la chirurgie (chirurgie programmée et chirurgie urgente ne pouvant être différée) [44, 109].

Dans une approche pragmatique, deux types d'interventions chirurgicales ou d'actes invasifs doivent être distingués : celles dites « à haut risque » où la fréquence et /ou la gravité des saignements péri-opératoires sont élevées, et celles dites « à risque faible ou modéré » ou le risque hémorragique est moindre. Une liste non exhautive des principaux actes de chirurgie orale, classé en fonction du risque hémorragique est présentée dans l'annexe 2. 


\section{CHIRURGIES OU ACTES INVASIFS A RISQUE HEMORRAGIQUE FAIBLE oU MODEREE}

Il s'agit des soins dentaires ou parodontaux ainsi que les actes chirurgicaux pour lesquels les pertes sanguines sont faibles (volume $<50 \mathrm{~mL}$, aucun cas de transfusion plaquettaire rapporté) [24, 21,239], non critiques par leur localisation et facilement contrôlables par des mesures simples d'hémostase mécanique.

\section{CHIRURGIES ou ACTES INVASIFS A RISQUE HEMORRAGIQUE ELEVE}

Il s'agit de tous les actes pour lesquels la probabilité d'un saignement est cliniquement significative (durée opératoire > 1 heure, abaissement du taux $\mathrm{Hb}$, transfusion plaquettaire rapportée), ou critique par leur localisation (sinus maxillaire, langue et plancher buccal) et/ou difficilement contrôlable par une hémostase chirurgicale conventionnelle en pratique de ville (compression, sutures, hémostatiques locaux à base de collagène, de gélatine ou de cellulose). La nécessité de recourir à une colle biologique, à une électrocoagulation mono-ou bipolaire, pour assurer l'hémostase du patient, est un critère utilisé pour classer les chirurgies comme à risque hémorragique élevé.

\section{LOCALISATION CRITIQUE}

En dehors de toute prise de traitement antithrombotique, des régions anatomiques font état de complications hémorragiques graves suites à des interventions de chirurgie orale (avulsion de la $3^{\text {ème }}$ molaire mandibulaire, pose d'implant en région symphysaire, élévation du sinus maxillaire, prélèvement symphysaire). Ainsi ont été rapportés :

- le sinus maxillaire avec le risque d'hémosinus suite à la lésion de l'artères alvéolaire postéro-supérieure ou antrale $[169,175]$;

- le plancher buccal avec le risque d'hématome compressif et/ou d'hémorragie grave suite à la lésion de l'artère linguale ou de l'une de ses branches (artère submentale ou submentonnière) [53, 75, 117, 179, 186, 220, 235].

Même si ces événements demeurent exceptionnels, la littérature rapporte régulièrement des cas d'hémorragies graves avec engagement du pronostic vital $[75,211,218]$ dont certaines fatales [107, 230].

\subsubsection{Facteurs de risque hémorragique susceptibles de majorer le risque hémorragique chirurgical.}

Plusieurs études cliniques ont mis en évidence des facteurs prédictifs d'une augmentation du risque d'événement de saignements péri-opératoires : ils sont en rapport soit avec l'âge du patient et ses co-morbidités, soit avec les conditions locales des tissus [72].

\section{ÂGE et COMORBIDITES}

Les études de phase II et de III, ainsi que les études observationnelles rapportant des hémorragies graves survenant chez les patients traités par antithrombotiques ont pu identifier des facteurs de risque responsables de surdosage $[4,122]$. Le score HAS-BLED peut être utile pour apprécier l'impact de ces facteurs sur le risque hémorragique en cas de chirurgie ou de geste invasif [246]. Parmi ces facteurs, l'âge et l'insuffisance rénale ainsi que les co-médications sont les plus souvent rapportés $[173,256,261]$. À noter que la fréquence de ces facteurs de risque est élevée dans la population des patients traités par antithrombotiques au long cours. L'association ou le cumul de plusieurs facteurs de risque impose une vigilance particulière et un contact préalable avec le cardiologue ou le médecin traitant. L'annexe 3 répertorie les principaux facteurs de risques hémorragiques.

\section{MÉDICAMENTS et ALIMENTATION}

Les prescriptions médicamenteuses peuvent induire de nombreuses interactions avec tous les antithrombotiques. La liste des médicaments formellement contre-indiqués ainsi que celle des médicaments majorant le risque hémorragique ou thrombotique, suceptibles d'être prescrits par le praticien, seront rappelées pour chaque famille d'antithrombotique.

L'alimentation, les jus de fruit et certaines plantes médicinales peuvent également interagir avec les AVK, influencer leur efficacité mais aussi être à l'origine de surdosage [81, 106, $271,285]$. Ces interactions ne doivent être ni méconnues, ni sous-estimées. La période postopératoire est une période critique car elle s'accompagne bien souvent d'une nouvelle prescription et/ou d'une modification des habitudes alimentaires. Un suivi clinique et/ou biologique est nécessaire en cas de prescription anti-infectieuse et/ou antalgique $[23,38,52,72$, $79,159,171,187,208,241,268,289,291,292]$.

\section{CONDITIONS LOCALES}

Une inflammation locale de la gencive (gingivite, parodontite...), la présence de foyers infectieux (granulome, kyste radiculodentaire, péricoronarite ....), du fait de la vasodilatation et de la perméabilité capillaire accrue, augmente potentiellement le risque d'évènement hémorragique [173, 221, 225, 245]. De même l'absence de gencive attachée rend la suture et la traction des tissus mous plus difficile à réaliser.

Le cumul de plusieurs facteurs de risque (âge et comorbidité, médicaments et alimentation, conditions locales défavorables) peut conduire à une situation clinique à haut risque hémorragique.

\subsection{2. À partir d'examen(s) biologique(s)}

Dans les études cliniques, les tests d'hémostase permettent de demontrer l'efficacité in vitro des antithrombotiques. Lorsque 
l'utilité d'une surveillance biologique est établie (AVK, héparines à doses curatives), les tests biologiques servent à gérer le risque hémorragique (surdosage) par adaptation posologique. Leur prescription en pré-opératoire pour prédire le risque hémorragique associé à l'acte chirurgical est fonction de la molécule antithrombotique et de la procédure invasive $[61,70]$.

A l'issue de la consultation pré-opératoire, le praticien doit :

pouvoir identifier les patients ou les situations cliniques à haut risque de complications hémorragiques ;

établir l'ensemble des mesures préventives nécessaire afin d'optimiser la sécurité hémostatique.

\subsection{Choix de la structure de prise en charge : en ville ou en milieu hospitalier?}

La prise en charge peut se faire en pratique de ville pour les antithrombotiques prescrits au long cours pour toutes les chirurgies ou actes invasifs programmées à risque hémorragique faible ou modéré.

Pour les situations cliniques où il existe un risque thrombotique élevé et/ou une chirurgie à haut risque hémorragique, la prise en charge sera hospitalière. Il en va de même pour les patients ayant une pathologie cardiovasculaire non stabilisée et/ou possédant d'autres anomalies constitutionnelles ou induites de l'hémostase. Ces cas particuliers imposent une hospitalisation, une concertation pluridisciplinaire et une hémostase spécifique à chaque cas [44, 109, 199].

\subsection{Hémostase locale}

Toute chirurgie ou acte invasif hémorragique se définit par une effusion de sang en dehors d'un vaisseau. L'obtention d'une hémostase en fin d'intervention doit être contrôlée. Il est à noter que la qualité de l'hémostase reste influencée en premier lieu par la technique chirurgicale et par la maîtrise du geste chirurgical [160].

- En cas d'extraction dentaire ou de pose d'implant dentaire, le saignement est facilement contrôlable par des mesures simples d'hémostase mécanique (sutures + compression). En l'absence d'anomalie congénitale et/ou acquise de l'hémostase, le délai d'hémostase est généralement obtenu en moins 10 minutes.

- En cas de plaie gingivale ou muqueuse, ces mêmes mesures mécaniques simples (sutures + compression) sont normalement suffisantes. En cas de prélèvement palatin, la pose d'une plaque palatine est une mesure simple permettant d'assurer une compression mécanique efficace.

- En cas de décollement muqueux important, il est nécessaire de disposer d'une électrocoagulation mono-ou bipolaire.

Lorsque ces techniques d'hémostase chirurgicales conventionnelles (mécaniques et/ou thermiques) sont insuffisantes, des hémostatiques locaux peuvent être utilisés en complément de l'hémostase.
Chez les patients sous antithrombotiques, afin de palier au surcoût hémorragique, l'utilisation systématique d'adjuvants hémostatiques comme les hémostatiques locaux et/ou d'acide tranexamique est consensuelle. Le temps d'obtention de l'hémostase, le volume des pertes sanguines, le taux de complications hémorragiques per- et postopératoire sont les critères le plus souvent utilisés pour évaluer l'efficacité de ces produits.

Les hémostatiques locaux utilisés en chirurgie orale regroupent les hémostatiques chirurgicaux et l'acide tranexamique.

\subsubsection{Hémostatiques chirurgicaux}

Par définition, les hémostatiques chirurgicaux sont utilisés en per-opératoire par le praticien pour empêcher la fuite de sang en cas de plaie vasculaire située sur des capillaires.

Il est important de rappeler que les hémorragies artérielles ou veineuses d'intensité élevée ne relèvent pas d'un traitement par ces produits.

Les hémostatiques chirurgicaux sont classés en deux groupes : les hémostatiques sans action spécifique sur la cascade d'évènements survennant au cours de l'hémostase et les hémostatiques ayant une action spécifique [231].

\subsubsection{Hémostatiques chirurgicaux sans action spécifique sur la cascade d'évènements survenant au cours de l'hémostase}

Ils regroupent des produits d'origine végétale à base d'oxycellulose (SURGICEL $\left.{ }^{\circledR}\right)$, des produits d'origine animale à base de collagène $\left(\right.$ PANGEN $\left.^{\circledR}\right)$ ou de gélatine (CURASPON ${ }^{\circledR}$ ). Ces produits ont un statut réglementaire de dispositif médical stérile (DMS). Ils se présentent sous forme d'éponges et de mèches stériles. Ces dispositifs sont prêts à l'emploi et se conservent à température ambiante. Ils agissent essentiellement par absoption du sang et par compression. Ils sont donc particulièrement efficaces en cas de tamponnement intra-alvéolaire. Au contact du sang, ils augmentent de volume, forment un bouchon gélatineux (collagène, gélatine) ou une masse brune (oxycellulose) qui comble la plaie et s'oppose mécaniquement à l'écoulement du sang [160].

L'utilisation de colle cyanoacrylate (HISTOACRYL ${ }^{\circledR}$ ) permet d'assurer une étanchéité vis-à-vis des fuites ou maintenir en place les hémostatiques chirurgicaux en cas de plaie gingivale en l'absence de sutures $[17,193]$.

\subsubsection{Hémostatiques chirurgicaux avec action spécifique sur la cascade d'évènements survenant au cours de l'hémostase.}

Il s'agit des colles biologiques également appelées « colles de fibrine ». Ces produits sont obtenus par fractionnement du plasma humain et ont le statut particulier de médicament dérivé du sang (MDS). En France, ces MDS sont réservés à 
l'usage hospitalier et compte tenu du risque de transmission d'agents infectieux conventionnels ou non, ils obéissent au décret de traçabilité n95-566 du 06 mai 1995.

Dans la littérature, seules deux colles sont utilisées en chirurgie orale [232]. Il s'agit du TISSUCOL KIT ${ }^{\circledR}$ et du BLERIPLAST ${ }^{\circledR}$ [2]. Elles sont utilisées sous forme gel liquide à l'aide d'applicateur. Elles contiennent des protéines coagulables d'origine humaine (fibrinogène, facteur XIII, fibronectine, thrombine) et de l'aprotinine d'origine bovine. Elles agisent sur l'hémostase en reproduisant la dernière étape de la coagulation. Le fibrinogène se transforme en fibrine sous l'action de la thrombine. Le facteur XIII va stabiliser le caillot et la dégradation protéolytique de la fibrine est inhibée par l'aprotinine. Peu d'études ont évalué l'intérêt des colles biologiques en chirurgie dento-alvéolaire chez des patients sous antithrombotiques [41, 45, 57, 126, 209]. Les études randomisées contrôlées disponibles n'ont pas permis de montrer un éventuel gain hémostatique dans la prévention des saignements postopératoires comparativement aux méthodes conventionnelles et/ou aux hémostatiques chirurgicaux usuels [41, 57, 126]. En revanche en cas de saignements per-opératoires non contrôlés par ces mesures, ou en cas de complications hémorragiques, l'utilisation de ces colles, en fin de procédure ou en cas de réintervention chirurgicale, permet d'améliorer la sécurité hémostastique [232].

\subsubsection{Acide tranexamique}

L'acide tranexamique (EXACYL ${ }^{\circledR}$ ) est le seul médicament antifibrinolytique possédant une AMM dans la prévention et le traitement des accidents hémorragiques entretenus par une fibrinolyse locale comme c'est le cas au décours des interventions stomatologiques, ORL et gynécologiques [151].

L'acide tranexamique est un analogue de la lysine qui se lie aux sites de liaison lysine du plasminogène et de la plasmine. Il bloque la fixation du plasminogène et de la plasmine sur la fibrine et empêche la dégradation de cette dernière. L'acide tranexamique est un inhibiteur compétitif de la fibrinolyse [89]. Plusieurs études in vitro ont montré qu'une concentration de $10 \mu \mathrm{g} / \mathrm{mL}$ d'acide tranexamique permet de réduit de $80 \%$ de l'activité enzymatique du t-PA (tissue plasminogen activator). Dans la cavité orale, le t-PA est libéré en grande quantité en cas de trauma opératoire par les cellules épithéliales de la muqueuse orale et de la salive $[118,182]$. L'efficacité de l'acide tranexamique dans la prévention et le traitement des saignements post-extractionnels a été démontrée lors de nombreux essais cliniques $[46,57,58,236,249$, 263, 269].

L'intérêt de ces hémostatiques locaux ainsi que leur place dans la stratégie de la gestion péri-opératoire du risque hémorragique en cas de maintien des antithrombotiques sera abordé dans un paragraphe spécifique pour chaque famille de médicaments (AAP, AVK, AOD, HBPM).

\subsection{Suivi et prise en charge post-opératoire}

\subsubsection{Surveillance et conseils post-opératoires}

Le suivi post-opératoire est important dans la prise en charge des patients. A l'issue d'une intervention de chirurgie orale, des conseils post-opératoires verbaux et écrits seront donnés aux patients. Cette surveillance postopératoire, doit être particulièrement rigoureuse en cas chirurgie à risque hémorragique élevé [74]. Des consignes pour l'hygiène buccodentaire et sur la conduite à tenir en cas de saignement postopératoire seront données.

La survenue d'une hémorragie post-opératoire peut avoir lieu immédiatement après intervention ou dans les jours suivants jusqu'à $J+6$. Une surveillance de la bonne cicatrisation muqueuse via un contrôle post-opératoire à $\mathrm{J}+10$ est indispensable. Le rapport bénéfice/risque de la poursuite des antithrombotiques en cas de chirurgie dento-alvéolaire est conditionné par une coordination optimale du parcours de soin et une bonne adhésion des patients (information, observance, éducation thérapeutique).

\subsubsection{Traitement curatif des complications hémorragiques}

Les complications hémorragiques sont définies par des saignements non contrôlables par les patients.

Elles peuvent être classées en deux catégories : les hémorragies mineures et les hémorragies graves. Les hémorragies mineures regroupent les suitements permanents, les caillots extra-alvéolaires et les ecchymoses. Les hémorragies graves correspondent aux saignements postopératoires importants et aux hématomes expansifs des espaces profonds (loge submandibulaire par exemple). Ces complications hémorragiques peuvent engager le pronostic vital du patient et nécessitent une prise en charge hospitalière. Elles sont exceptionnelles en chirurgie orale et sont toujours en rapport avec un traumatisme invasif des tissus mous et/ou une section artériolaire ou artérielle.

Les complications hémorragiques chez les patients sous antithrombotiques après une chirurgie orale sont pour l'essentiel de bon pronostic. Leurs prises en charges reposent toujours en premier lieu sur une révision de la plaie opératoire avec une reprise de l'hémostase locale. La recherche d'une cause locale du saignement est impérative. La zone opératoire est réouverte après anesthésie, le matériau de compression intraalvéolaire est retiré. Ce geste permet de visualiser l'origine du saignement pour agir directement à sa source. La suite est identique à la prise en charge initiale. Un matériau hémostatique intra-alvéolaire est remis en place, des sutures sont réalisées, une compression locale est instaurée et un agent antifibrinolytique par voie locale peut être associé au cours de la période de cicatrisation. L'absence de contrôle durable de l'hémorragie par les mesures usuelles d'hémostase locale est considérée comme un critère de gravité et une prise en charge 
hospitalière est alors nécessaire. Le recours à des hémostatiques généraux et une surveillance médicale peuvent être indiqué [71, 194, 207].

\section{Spécificités de la prise en charge d'un patient traité par agent(s) antiplaquettaire(s)}

\subsection{Les enjeux}

Les maladies cardiovasculaires liées à l'arthériosclérose constituent la première cause de mortalité dans les pays industrialisés et la deuxième cause dans le monde.

En France, la prévalence de la maladie de coronarienne est de 3,9\%. Les syndromes coronariens aigus (SCA) sont responsables de 150000 hospitalisations par an et 46000 décès par an [154]. Les accidents vasculaires cérébraux (AVC) ischémiques et les accidents ischémiques transitoires (AIT) présentent une incidence estimée à 130000 cas par an et sont à l'origine de 40000 décès par an. Cette pathologie est la première cause de handicap non traumatique de l'adulte. Le risque de récidive d'AVC à 5 ans est estimé entre 30 et $43 \%$ [155]. L'artériopathie oblitérante des membres inférieurs (AOMI) concerne environ 800000 patients, et les deux tiers sont symptomatiques. L'incidence de cette pathologie est de 90000 nouveaux cas par an et est responsable de 60000 hospitalisations et de 10000 amputations [31, 156].

Les agents antiplaquettaires (AAP) sont indiqués en prévention secondaire dans la maladie athéromateuse, afin de prévenir la survenue d'évènements cardiovasculaires [8, 78].

La prescription d'une monothérapie antiplaquettaire à vie (aspirine ou clopidogrel) est recommandée chez les patients ayant présenté un SCA (en l'absence d'indication d'un traitement anticoagulant ou d'une contre-indication formelle à l'aspirine et/ou au clopidogrel), un AVC ischémique ou une AOMI [8]. Une bithérapie antiplaquettaire (aspirine + clopidogrel ou prasugrel ou ticagrelor) est justifiée en cas de risque thrombotique majeur. Elle est recommandée durant l'année qui suit un SCA, en particulier un infarctus du myocarde (IDM) [13]. La durée optimale d'une bithérapie est controversée car des études rapportent la survenue d'accidents thrombotiques suite à l'arrêt prématurée de la thiénopyridine associée [251, 252]. La durée minimale de prescription d'une bithérapie est de 1 à 3 mois après une angioplastie carotidienne ou intracrânienne, de 1 mois après la pose d'un stent nu [270] et de 6 à 12 mois après la pose d'un stent actif $[8,121]$. Au-delà de ces délais, la poursuite ou non de la bithérapie est à adapter au cas par cas mais n'est généralement pas recommandée [13, 252].

L'aspirine seule est recommandée, en prévention primaire lorsque le risque cardiovasculaire est élevé en particulier chez le diabétique [8].

En France, la prescription d'AAP concerne près de $5 \%$ de la population [27]. Le nombre de nouveaux patients par an est estimé entre 200000 et 300 000. En 2010, $81 \%$ des patients traités par AAP étaient sous aspirine et 1 million de patients étaient traités par clopidogrel [27]. Le nombre de patients par AAP ne cesse de croître, en raison de l'effet combiné du vieillissement de la population, de l'extension de leurs indications et de l'essor des thérapeutiques interventionnelles. D'après les données nationales du programme de médicalisation des systèmes d'information (PMSI), 125000 patients ont bénéficié $d^{\prime}$ une intervention coronaire percutanée (ICP) en 2007 [154].

Seuls les AAP prescrits par voie orale dans le cadre d'un traitement ambulatoire font l'objet de recommandations (voir annexe 1). Les antagonistes des récepteurs GPIIb/ IIIa (abciximab, eptifibatide, tirofiban) indiqués dans la prise en charge initiale des SCA, ne seront pas étudiés. La réalisation d'une chirurgie orale n'est en principe pas indiquée durant cette période et sera reportée de fait à distance de l'arrêt des antiGPIIb-IIIa.

\subsection{Les agents antiplaquettaires}

\subsubsection{Les agents antiplaquettaires classiques}

Les AAP classiques regroupent l'aspirine, le dipyridamole, la ticlopidine et le clopidogrel.

\subsubsection{Aspirine}

L'acide acétylsalicylique ou aspirine bloque la cyclo-oxygénase-1 (COX-1) de la plaquette, ce qui inhibe la production de thromboxane A2 (TXA2), un puissant vasoconstricteur et inducteur de l'aggrégation plaquettaire. Cette inhibition étant irréversible, l'action de l'aspirine sur les plaquettes est permanente durant toute leur durée de vie. L'inhibition de la COX-1 est complète sous faibles doses d'aspirine (75 à $160 \mathrm{mg} / \mathrm{jour}$ ) ou après une dose de charge de $325 \mathrm{mg}$. Des doses quotidiennes supérieures à $100 \mathrm{mg}$ en clinique n'augmentent pas l'effet antiaggrégant plaquettaire, mais accroissent le risque d'hémorragie digestive spontanée. Après interruption de l'aspirine, l'agrégabililité plaquettaire se normalise en 5 à 7 jours (en moyenne $+10 \%$ de plaquettes par jour) $[78,91]$.

L'aspirine est l'AAP le plus utilisé. Elle est commercialisée soit seule (KARDEGIC ${ }^{\circledR}$, CARDIOSOLUPSAN ${ }^{\circledR}$ ) $[133,145]$, soit en association avec le dipyridamole (ASASANTINE ${ }^{\circledR}$ ) [142], le clopidogrel (DUOPLAVIN ${ }^{\circledR}$ ) [141] ou la pravastatine (PRAVA$\left.\operatorname{DUAL}^{\circledR}\right)[143]$.

Deux méta-analyses ont évalué l'efficacité antithrombotique de l'aspirine versus placebo [21-22]. En prévention primaire, l'aspirine permet de réduire de $12 \%$ les évènements cardiovasculaires, la plus forte réduction portant sur les IDM non mortels. Son effet est faible et non significatif sur la mortalité coronnarienne et sur les AVC. En prévention secondaire, l'aspirine permet une réduction significative du risque de récidives 
d'IDM de $26 \%$, et d'AIT/AVC ischémique de $25 \%$. Elle est associée également à une baisse de $15 \%$ des décès d'origine cardiovasculaire. Dans le cadre d'une thérapeutique de revascularisation, l'intérêt de l'aspirine après pose de stent est double: réduction signifivicative du nombre d'évènements cardiovasculaires et baisse du taux de thromboses sur stent [8].

Tous les patients ne réagissent pas avec la même intensité aux AAP. Le taux de faibles répondeurs à l'aspirine est particulièrement important chez les diabétiques de type 1 et dans le sexe féminin [8]. Le taux moyen de non répondeurs se situe aux environs de $6 \%$ de la population [69]. Les individus dits résistants à l'aspirine ont souvent un blocage efficace de la production de thromboxane A2 (TXA2), compensé par une augmentation de la stimulation des autres voies d'activation (récepteur ADP, récepteur thrombine). Ils conservent ainsi une agrégabilité plaquettaire importante malgré l'activité inhibitrice de l'aspirine.

Chez les patients sous anti-inflammatoires non stéroïdiens (AINS) au long court et devant bénéficiés d'une cardioprotection par antiplaquettaire, il est à noter que l'association d'ibuprofène diminuerait de $70 \%$ la protection cardiovasculaire de l'aspirine (OR 1,73, IC à $95 \% 1,05$ à 2,84, p < 0 ,05), en bloquant l'accès de l'aspirine à la $\mathrm{COX}-1$ par simple phénomène de compétition au niveau du recepteur [203].

\subsubsection{Dipyridamole}

Le dipyridamole ralentit la recapture de l'adénosine monophosphate (AMP) par les plaquettes. Il est décrit aussi comme inhibiteur de la phosphodiestérase de l'AMP cyclique (AMPC). Ces actions contribuent à une élévation intracellulaire en $A M P c$, second messager anti-activateur plaquettaire.

Le dipyridamole est commercialisé en France seul à la dose de $75 \mathrm{mg}$ (CLERIDIUM ${ }^{\circledR}$, PERSANTINE ${ }^{\circledR}$ ) [132], $150 \mathrm{mg}$ (CLERIDIUM $^{\circledR}$ ) ou à la dose de $200 \mathrm{mg}$ combinée à $25 \mathrm{mg}$ d'aspirine (ASASANTINE LP ${ }^{\circledR}$ ) [142].

L'efficacité clinique du dipyridamole en monothérapie est faible et cet AAP est donc peu prescrit [8]. Une méta-analyse récente a souligné l'intérêt de l'association aspirine + dipyridamole dans la prévention secondaire des AVC ischémiques par rapport au placebo et à l'aspirine (réduction du risque relatif de $39 \%$ et $22 \%$ respectivement) [192].

\subsubsection{Ticlopidine}

La ticlopidine a été la première thiénopyridine synthétisée. Comme toutes les thiénopyridines, elle inhibe de façon irréversible le récepteur purinergique $\mathrm{P} 2 \mathrm{Y} 12$ à l'ADP, un facteur d'activation et d'agrégation plaquettaire, déclenchée par l'ADP [91]. La ticlopidine est commercialisée en France depuis 1978, à la dose de $250 \mathrm{mg}\left(\right.$ TICLID $\left.^{\circledR}\right)$ à raison de deux prises par jour [150]. Un délai de 8 à 11 jours est nécessaire pour obtenir une inhibition maximale de l'agrégabilité plaquettaire. Son efficacité antithrombotique est certaine mais ses effets secondaires expliquent qu'il a été presque totalement abandonné au profit du clopidogrel. Une surveillance de la numération formule sanguine est recommandée en raison du risque élevé, pendant les trois premiers mois de traitement, d'agranulocytose et de thrombopénies observées chez $2,4 \%$ des patients [8].

\subsubsection{Clopidogrel}

Le clopidogrel est actuellement la thiénopyridine la plus prescrite [8]. Le clopidgrel est plus puissant que la ticlopidine avec une meilleure pharmacocinétique (une prise par jour au lieu de deux) et un meilleur profil de tolérance (rares cas de leucopénie et de thrombocytopénie) [8]. Le clopidogrel est une prodrogue qui impose un premier passage hépatique avec biotransformation au niveau des CYP 450. C'est un inhibiteur non compétitif irréversible du récepteur ADP P2Y12. Bien qu'irréversible, l'inhibition n'est pas totale : la réduction de l'agrégation plaquettaire est de 40 à $60 \%$ [91]. Le clopidogrel (PLAVIX ${ }^{\circledR}$ ) est utilisé depuis 1998 , à la dose de $75 \mathrm{mg}$ par jour [135]. En monothérapie, le clopidogrel a démontré une supériorité comparativement à l'aspirine uniquement dans la prévention secondaire des AIT/AVC ischémiques [135]. Les modes d'action de l'aspirine et du clopidogrel étant différents, leur combinaison suggère une grande efficacité antithrombotique. La supériorité de la bithérapie comparativement à l'aspirine seule a été démontrée dans deux situations : les patients avec un antécédent d'IDM de moins de 1 an et après revascularisation coronaire. Dans l'étude CURE (Clopidogrel in Unstable Angina to Prevent Recurrent Ischemic Events) [196, 213], l'association aspirine + clopidogrel a permis de réduire le critère principal combinant (décès cardiovasculaire/IDM/AVC) et le nombre d'IDM non mortel respectivement de $-2,1 \%$ et $1,5 \%$ versus aspirine seule. Une bithérapie pendant 1 mois après la pose de stent nu, hors contexte IDM, d'après les études COMMIT (Clopidogrel and metoprolol in Myocardial Infarction Trial) [62] et CLARITY (Clopidogrel as Adjunctive Reperfusion Therapy) [253], permet d'obtenir une diminution significative de $-0,6$ à $-2,5 \%$ du critère composite (décès cardiovasculaire/ IDM/AVC) comparativement à l'aspirine seule. Plusieurs études randomisées $[8,13,76,222]$ ont montré l'intérêt d'une bithérapie pendant les 6 à 12 premiers mois qui suivent la pose de stents actifs ou d'un pontage veineux, par une diminution significative du taux de thrombose sur stent, un meilleur maintien de la perméabilité du greffon veineux, ainsi qu'une baisse de la mortalité. La durée optimale de la bithérapie reste controversée [13].

L'activité antiplaquettaire du clopidogrel est sujette à une grande variabilité interindividuelle avec un taux de non répondeurs estimé de 12 à $35 \%$ [91]. Plusieurs phénomènes sont incriminés: un polymorphisme génétique, des interactions médicamenteuses, le diabète de type 1 , l'insuffisance rénale et un âge avancé [69]. Parmi les nombreuses interactions 
médicamenteuses, deux seules ont un impact clinique bien décrit: l'atorvastatine et l'omeprazole. L'administration concomitante de l'une de ces molécules avec le clopidogrel diminue l'efficacité de ce dernier de $25 \%$ avec une augmentation significative du risque cardiovasculaire des patients. A noter que cet effet n'a pas été retrouvé avec les autres inhibiteurs de la pompe à protons (lanzoprazole, pantoprazole...) et autres statines $[8,282]$.

\subsubsection{Les nouveaux agents antiplaquettaires}

Le traitement des SCA et les patients coronariens traités par angioplastie bénéficient d'une bithérapie aspiririne + clopidogrel mais il a été démontré une hétérogénéité de la réponse à ces molécules et les patients hyporépondeurs présentent un sur-risque d'évènement ischémique. C'est pourquoi de nouvelles molécules permettant une inhibition plus rapide, plus intense et plus réproductible ont été développées [88, 127]. Parmi celles-ci, deux sont actuellement commercialisées : le prasugrel et le ticagrelor.

\subsubsection{Prasugrel}

Le prasugrel, est une thienopyridine de $3^{\text {ème }}$ génération, qui présente deux avantages essentiels comparativement à la ticlopidine et au clopidogrel : un début d'action plus rapide (30 minutes après la prise) et une activité plus puissante [275]. Le prasugrel bloque le récepteur P2Y12 sensible à l'ADP, de manière irréversible, comme la ticlopidine et le clopidogrel, mais avec une inhibition plaquettaire moins variable et plus prédictive [91]. Le prasugrel (EFIENT ${ }^{\circledR}$ ) a obtenu son AMM en 2010 et est utilisé avec une dose de charge de $60 \mathrm{mg}$ et une dose d'entretien de $10 \mathrm{mg}, 1$ fois/jour [139].

Le prasugrel est plus efficace que le clopidogrel, en termes de protection des IDM et thromboses sur stents, mais au prix d'accidents hémorragiques plus nombreux. C'est la raison pour laquelle il est contre-indiqué en cas d'AVC et qu'une dose plus faible $(5 \mathrm{mg})$ est préconisée en cas de poids $<60 \mathrm{Kg}$, d'âge $>$ 75 ans et d'insuffisance rénale sévère [8]. L'étude TRITONTIMI38 (TRial to Assess Improvement in Therapeutic Outcomes by Optimizing Platelet InhibitioN with Prasugrel) $[219,286]$ a comparé l'efficacité et la tolérance du prasugrel (dose de charge de $60 \mathrm{mg}$ suivie d'une dose d'entretien de $10 \mathrm{mg} / \mathrm{j}$ ) au clopidogrel (dose de charge de $300 \mathrm{mg}$ suivie d'une dose de $75 \mathrm{mg} /$ j) chez 13608 patients sous bithérapie (aspirine + prasugrel ou clopidogrel) admis pour SCA avec un profil de risque élevé d'infarctus du myocarde avec élévation du segment ST (STEMI) ou intermédiaire (angor instable), devant bénéficier d'une ICP. Les résultats montrent que pour 1000 patients traités par le prasugrel, 23 infarctus ont été évités pour un surcroît de 6 complications hémorragiques majeures par rapport au clopidogrel. Le prasugrel diminue davantage le risque de récidive d'IDM et de thrombose de stent, quel que soit le type de stents, par rapport au clopidogrel. Le prasugrel est particulièrement efficace chez les diabétiques et chez les non répondeurs au clopidogrel. Le taux de non répondeurs au prasugrel n'est que de $3 \%$. En revanche, le prasugrel présente un sur-risque d'hémorragie majeure par rapport au clopidogrel $(2,4 \%$ au lieu de $1,8 \%)$. Les personnes âgées ( $>75$ ans), les personnes de faible poids $(<60 \mathrm{Kg}$ ) et les patients souffrant d'AIT/AVC ischémiques (risque d'hémorragie intra-crânienne) sont particulièrement exposés au risque de saignement spontané [8]. Le prasugrel est indiqué en association avec l'acide acétylsalicylique dans la prévention des événements athérothrombotiques chez les patients avec un SCA traité par une ICP primaire ou retardée [139, 282].

\subsubsection{Ticagrelor}

Le ticagrelor est un AAP d'une nouvelle classe chimique, la famille des cyclo-pentyl-triazolo-pyrimidines. C'est un inhibiteur direct et réversible du récepteur plaquettaire $P_{2} Y_{12}$ de l'ADP. Ce n'est pas une pro-drogue et il n'a pas besoin d'être métabolisé pour être actif $[91,215]$. Ces deux propriétés pharmacologiques le distinguent donc des thiénopyridines [215]. Les études de pharmacodynamie ont démontré que le ticagrelor a un pouvoir d'inhibition plaquettaire plus puissant, plus rapide et avec une moindre variabilité interindividuelle comparé au clopidogrel [284].

Le ticagrelor (BRILIQUE ${ }^{\circledR}$ ) est commercialisé en France depuis 2010 et est utilisé à la dose de 90 mg, 2 fois/jour après une dose de charge de $180 \mathrm{mg}$ [146]. Le ticagrelor a obtenu son AMM après les résultats de l'étude PLATO (The Study of Platelet Inhibition and Patients Outcomes) [174] effectuée chez 18624 patients admis pour SCA. Le ticagrelor s'est révélé légèrement plus efficace que le clopidogrel pour diminuer le risque de décès (RR 0,78, IC à $95 \%$ de 0,69 à 0,89, p<0,05), d'IDM après SCA (RR 0,84 , IC à $95 \%$ de 0,75 à $0,95, p<0,05$ ), de thrombose sur stent (RR 0,75, IC à $95 \%$ de 0,59 à $0,95, p<$ $0,05)$, mais non pour modifier le taux d'AIT/AVC ischémiques (RR 1,18, IC à $95 \%$ de 0,91 à 1,52, ns). Son efficacité est diminuée lorsqu'il est associé à de fortes doses d'aspirine (> $300 \mathrm{mg} /$ jour). Il n'a pas d'effet préférentiel chez le patient diabétique. Le risque d'hémorragie majeure (hémorragie intracrânienne ou digestive, perte de 30 à $50 \mathrm{~g} / \mathrm{l}$ d'hémoglobine) n'est pas différent entre ticagrelor (11,6\%/an ) et clopidogrel $(11,2 \% / a n)(p=0,433)$. Le ticagrelor en association avec aspirine est indiqué dans la prévention des événements athérothrombotiques chez les patients adultes ayant un SCA (angor instable, NSTEMI ou STEMI), incluant les patients traités médicalement et ceux traités par une ICP ou un pontage coronaire [146].

\subsection{Arrêt ou maintien du traitement par agent antiplaquettaire?}

- De nombreuses études rétrospectives rapportent la survenue de SCA, d'IDM, d'AVC ischémiques et de décès chez des 
patients ayant interrompu leur traitement par aspirine durant la période postopératoire immédiate et dans le mois suivant $[25,30,60,64,65,102,103,112,116,127,214$, 277].

Dans une méta-analyse [40], l'arrêt de l'aspirine chez un patient sous aspirine au long cours est associé une augmentation du risque d'évènement thrombotique (cardiaque ou neurologique) d'un facteur trois (0R 3,14, IC à $95 \%$ de 1,75 à $5,61, p<0,05)$. Ce risque d'événement indésirable thrombotique est encore plus élevé chez les patients ayant eu une implantation sous stent coronaire (OR 89,78, IC à $95 \%$ de $29,90$ à $269,60, p<0,01)$.

De plus, Minassian et coll., 2010 [216] rapportent dans une enquête observationnelle rétrospective de patients ayant un antécédent de SCA ( $n=650$ patients) ou d'IDM $(n=525)$, une augmentation significative du nombre d'événement thrombotique durant les 4 semaines qui suivent un traitement dentaire (0R 1,5, IC à $95 \%$ de 1,09 à 2,06, p < 0,05). Des observations $[165,166]$ similaires sont rapportées avec un arrêt prématuré ou inaproprié du clopidogrel.

L'ensemble des données de la littérature soulignent le danger potentiel avec une augmentation de la morbimortalité en cas d'arrêt du traitement par AAP chez les coronariens ou chez les patients ayant une pathologie vasculaire cérébrale en vue d'une intervention chirurgicale.

Les complications thrombotiques indésirables ne sont pas immédiates et suivent l'interruption de l'aspirine et/ou du clopidogrel dans un délai moyen de 8 à 25 jours. L'effet délétère de l'arrêt du traitement par AAP s'expliquerait non seulement par la suppression de la protection antiplaquettaire mais aussi par l'existence d'un phénomène de rebond de l'activité plaquettaire à l'arrêt des AAP [201].

La poursuite du traitement par AAP avant une intervention chirurgicale permet d'assurer la prévention du risque thrombotique. En contrepartie, cette stratégie thérapeutique majore le risque de saignement péri-opératoire.

Le risque de saignement postextractionnel en cas de maintien du traitement par aspirine ou clopidogrel moyen est estimé à moins de $2 \%$ avec des valeurs extrêmes allant de 0 à $18 \%$ selon les études (voir annexe 4). Pour l'ensemble de ces études cliniques [24, 36, 48, 50, 54, 56, 113, 185, 197, 204, 228, 234, $248,281]$, aucune complication hémorragique sévère non contrôlable par des mesures d'hémostase locale n'est rapportée. Deux études [212, 237] ont évalué la perte sanguine après extraction dentaire chez des patients sous aspirine ou clopidogrel comparativement à des patients ne prenant pas d'aspirine ou ayant interrompu leur traitement par AAP 7 jours avant la procédure dentaire ou sous AINS. Même si les deux études signalent une plus grande perte sanguine chez les patients sous AAP comparativement aux groupes contrôles, il n'existe pas de différence significative. De plus, aucune différence en terme d'incidence de saignement postopératoire entre les groupes n'est rapportée.
En cas de bithérapie, l'incidence de saignement avec l'association aspirine + dipyridamole est similaire à celle observée avec l'aspirine seule [228, 278]. En revanche, en cas d'association aspirine + clopidogrel, le risque de saignement, durant la période postopératoire immédiate est plus élevé en cas de bithérapie comparativement à l'aspirine seule (RR 28, $p<0,001)$ et au clopidogrel ( $R R 24, p<0,001)[54,197]$ mais facilement contrôlable par des mesures d'hémostase locale [36, 54, 197, 227, 228, 234]. Aucune hémorragie sévère ou tardive n'est rapportée. Une hémostase chirurgicale simple associant hémostatique local et sutures permet de contrôler efficacement le risque de saignement péri-opératoire. Par ailleurs, des études $[165,166]$ ont montré chez des patients porteurs de stents, la survenue d'événement thrombotique dans une période de 1 à 3 mois suite à l'arrêt de la bithérapie (arrêt du clopidogrel et poursuite de l'aspirine). Ceci soulève la question d'un possible « phénomène de rebond prothrombotique » à l'arrêt du clopidogrel [257], effet délétère également évoqué avec l'aspirine $[60,102,103]$ mais dont la physiopathologie n'est toujours pas élucidée [18].

- L'ensemble des études cliniques montrent que les extractions dentaires peuvent être réalisées sous aspirine ou clopidogrel en monothérapie (voir annexe 5) et en association (voir annexe $\underline{6}$ ) sans modification du traitement. Les données scientifiques concernant la ticlopidine et l'association aspirine + dipyridamole sont très pauvres, il n'en ressort pas de différence statistiquement significative en terme de risque hémorragique comparativement à l'aspirine et au clopidogrel. Concernant le prasugrel et le ticagrelor, aucune donnée n'est publiée, ces molécules, étant exceptionnellement prescrites en monothérapie.

Aucune donnée bibliographique n'est actuellement disponible concernant le risque de saignement associé à la pose d'implant chez un patient sous AAP. Dans l'état actuel des connaissances, la pose d'implant est un acte à risque hémorragique faible à modéré en l'absence de décollement mucopériosté dépassant la ligne de jonction muco-gingivale. Malgré l'absence de données de la littérature, la pose d'implant(s) dentaire(s) peut être pratiquée sans interrompre la thérapeutique antiplaquettaire (monothérapie ou bithérapie), compte tenu du fait la facilité d'accès de la zone opératoire et de l'efficacité de l'hémostase locale.

- En cas de chirurgie orale plus invasive (ex : chirurgie préimplantaire, chirurgie des kystes et tumeurs bénignes des maxillaires), aucune donnée bibliographique n'est disponible à ce jour. Pour ce type de chirurgie, comme le risque de saignement est plus élevé ou plus difficilement contrôlable, le maintien de la thérapeutique antiplaquettaire doit être discuté au cas par cas, en tenant compte de la balance bénéfice/risque (thrombotique et hémorragique) entre le praticien et le médecin prescripteur. Deux stratégies de gestion de la bithérapie peuvent être proposées : maintien de la bithérapie ou arrêt partiel de la bithérapie (arrêt du 
clopidogrel ou prasugrel ou ticagrelor et poursuite de l'aspirine) $[8,60,127,162,163]$.

\subsection{Quel test biologique peut-on utilisé pour évaluer le risque hémorragique chirurgical chez les patients sous agent antiplaquettaire ?}

Historiquement, le temps de saignement (TS) était utilisé pour le bilan de l'hémostase pré-opératoire ainsi que pour l'exploration d'un syndrome hémorragique. Même si de nombreuses études [24, 49, 240] ont mis en évidence un allongement du TS chez les patients traités par AAP, des études systématiques datant de 1990 avaient montré que le TS avait une valeur prédictive du risque hémorragique très faible $[198,250]$. Depuis 1998, en France, la prescription d'un TS en pré-opératoire n'est plus recommandée [161]. De nombreuses sociétés savantes $[61,70,265]$ ont depuis avalisé cette recommandation. Le TS (quelle que soit la méthode d'exploration : Duke ou Ivy) n'est plus inscrit à la nomenclature des actes de biologie médicale (NABM) depuis février 2013 [177]. D'autres tests sont proposés à l'heure actuelle pour surveiller les traitements antiplaquettaires. Il en existe deux familles principales : les tests d'aggrégation qui mesurent la réactivité des plaquettes à la stimulation d'un agoniste (acide arachidonique, adénosine diphosphate ADP), et ceux qui mesurent plus spécifiquement l'effet d'un médicament sur sa cible moléculaire (taux de thromboxane B2 urinaire pour l'aspirine, test VASP pour le clopidogrel). Dans le premier cas, les tests permettent de dire si les plaquettes du patient sont plus ou moins réactives lors d'une stimulation par un agoniste. Dans le second cas, les tests permettent de mesurer l'état d'inactivation de la cible moléculaire des médicaments prescrits, COX 1 pour l'aspirine, récepteur P2Y12 pour le clopidogrel et les autres inhibiteurs de ce récepteur. L'intérêt de ces tests d'inhibition plaquettaire est d'identifier les patients «mauvais répondeurs » ou « résistant aux AAP ». Pour l'heure, aucun de ces tests n'a fait la preuve de son utilité dans l'évaluation du risque hémorragique chirurgical chez des patients sous AAP et ils ne sont pas recommandés en routine.

\subsection{Quelle hémostase locale doit-on réaliser pour contrô- ler le risque hémorragique chirurgical ?}

Les mesures d'hémostase conventionnelles (sutures, compression, électrocoagulation) sont indispensables.

- En cas d'extraction simple (unitaire et multiple), les mesures d'hémostase conventionnelles (sutures + compression mécanique de 30 minutes) sont efficaces et suffisantes pour contrôler le saignement postopératoire chez les patients sous aspirine [24, 50, 185, 204, 281] ou sous clopidogrel $[54,197]$ avec un taux de saignement postopératoire résiduel acceptable d'environ 2 à $3 \%$. Sous bithérapie aspirine + clopidogrel, une hémostase locale associant sutures + compression semble insuffisante [197] et le recours à des hémostatiques locaux (éponge de collagène, mèche d'oxycellulose, colle de fibrine) $[36,54,227,234,239,248]$. En cas de bithérapie, un taux plus élevé de saignement est observé durant la première heure postopératoire [197, 234, 239]. Malgré l'absence de données de la littérature, en cas de pose d'implant(s), la réalisation de sutures et d'une compression digitale sont suffisantes pour assurer le contrôle d'hémostase.

- En cas d'extraction chirurgicale, le recours à des hémostatiques chirurgicaux (éponge de gélatine, fibrine, collagène, mèche $d^{\prime}$ oxycellulose) en complément aux mesures d'hémostase conventionnelle est recommandé, que le patient bénéficie d'une monothérapie [113] ou d'une bithérapie antiplaquettaire [54, 197, 227]. Pour des chirurgies à risque hémorragique élevé avec des décollements muqueux importants, des mesures similaires doivent être appliquées.

L'ensemble de ces travaux est présenté sous forme de tableaux synthétiques en annexe 5 et en annexe 6 .

\section{6 . Traitement curatif des complications hémorragiques}

La prise en charge d'une complication hémorragique repose toujours sur une reprise de l'hémostase locale. Elle est le plus souvent identique à la prise en charge initiale. Si le recours à des hémostatiques chirurgicaux à titre préventif n'est pas systématique chez les patients sous AAP, l'emploi de ces produits en présence d'une complication hémorragique est indispensable. Aucune complication hémorragique sévère (c'est-à-dire non contrôlable par des mesures d'hémostase locale) n'est rapporté dans la littérature. Il n'existe aucune donnée sur le prasugrel et le ticagrelor.

Dans le cas d'une complication hémorragique exceptionnellement non contrôlable par une reprise de l'hémostase locale, des auteurs préconisent l'utilisation de desmopressine ou de transfusion plaquettaire. Aucune étude clinique ne valide actuellement de telles mesures.

Si le recours à des hémostatiques chirurgicaux à titre préventif n'est pas systématique, l'emploi de ces produits en présence d'une complication hémorragique est indispensable.

\section{Spécificités de la prise en charge des patients traités par antivitamines $\mathrm{K}$}

\subsection{Les enjeux}

La maladie thrombo-embolique veineuse (MTEV) regroupe la thrombose veineuse superficielle (TVS), la thrombose veineuse profonde (TVP) et l'embolie pulmonaire (EP). La TVS est une pathologie bénigne, alors que la TVP et sa complication l'EP, par migration embolique de thrombus dans la circulation artérielle pulmonaire, sont associées à une 
morbidité et mortalité importantes [4]. La MTEV est une maladie fréquente. En France, l'incidence de la TVP et de l'EP sont estimées respectivement à 1,24/1 000 habitants/an et $0,6 / 1000$ habitants/an [233]. Les TVP et EP sont responsables de 10000 décès/ an et de $10 \%$ des décès hospitaliers $[4,80,264]$. De nombreuses chirurgies sont associées à un risque élevé de TVP en l'absence de traitement prophylactique. Il s'agit principalement de la chirurgie orthopédique et traumatologique, de la chirurgie abdominale, urologique et gynécologique majeures [80, 264].

Les cardiopathies emboligènes sont, dans $20 \%$ des cas, la cause d'AVC ischémiques [155]. Les principales sources cardiaques d'emboles sont : l'arythmie cardiaque par fibrillation auriculaire (ACFA) [157], les cardiopathies valvulaires et l'IDM compliqué [158].

L'ACFA est l'arythmie cardiaque la plus fréquente. Sa prévalence est estimée à 1 à $2 \%$ de la population et concerne entre 750000 et 1 million de patients en France. Elle croît avec l'âge, les deux tiers des patients ont entre 75 et 85 ans [157]. Les données nationales issues du PMSI de 2007 rapportent 84000 hospitalisations par an avec un diagnostic principal de ACFA et un chiffre de 349000 lorsque l'ACFA est codée comme diagnostic secondaire [191]. La fréquence de survenue d'AVC ischémique chez les patients atteints d'ACFA varie de moins de $2 \%$ par an (chez les patients sans risque cardiovasculaire associé) à plus de $10 \%$ par an (en cas antécédent d'AIT/AVC, hypertension artérielle, insuffisance cardiaque, âge $>75$ ans) $[129,210]$. Le risque thrombomembolique est évalué à l'aide des scores CHADS2 et CHA2DS2-VASc [190, 210]. L'aspirine ne fait plus partie des options thérapeutiques [210].

Les cardiopathies valvulaires (valvulopathies et prothèses valvulaires) ont une prévalence estimée à moins de $2 \%$ de la population générale et croît également avec l'âge (prévalence comprise entre 10 et $15 \%$ chez les patients de plus de 75 ans) [158]. Les patients présentant un rétrécissement mitral serré en ACFA ou porteurs d'une prothèse valvulaire sont à haut risque d'accident thrombotique [158]. Chaque année, 100000 patients sont atteints d'infarctus du myocarde (IDM) et près de $13 \%$ des patients pris en charge décèdent au cours de la première année [154]. Le risque d'AVC ischémique est majeur durant les 6 premiers mois qui suivent l'IDM. A distance de l'IDM, le risque d'AVC est d'environ 1,5\% par an. Certaines situations, comme la persistance d'un thrombus mural, l'existence d'anévrysme de la paroi ventriculaire ou de zone akinétique franche, d'une insuffisance cardiaque et d'un trouble du rythme associé, sont particulièrement thrombogènes (risque thrombotique annuel estimé entre 4 et $7 \%$ ) [158].

Les AVK sont indiqués dans la prévention de ces accidents thrombo-emboliques et sont habituellement prescrits en relais d'un traitement initial par l'héparine, anticoagulant injectable et plus adapté lors de la phase aiguë [9]. Les anticoagulants n'exercent aucune action directe sur un thrombus déjà formé ni sur les lésions tissulaires ischémiques. Toutefois, dans les cas où le thrombus est formé, l'administration d'anticoagulants vise à empêcher le caillot de grossir et à prévenir les complications thromboemboliques secondaires qui pourraient entraîner des séquelles graves et même d'issue fatale.

Plus des deux tiers des prescriptions des AVK relèvent de la cardiologie (valvulopathies, troubles du rythme, syndromes coronariens), tandis que moins de $20 \%$ relèvent de la pathologie veineuse thrombo-embolique. Dans les indications relevant de la cardiologie, le traitement est prescrit le plus souvent à vie. Dans les indications pour la MTEV, le traitement est généralement plus court, schématiquement de 3 mois à 1 an [262]

Quatre pour cent de la population française bénéficie d'un traitement anticoagulant. En 2013, plus d'1 million de patients ont été traités par AVK [10].

Dans la très grande majorité des cas, le traitement est prescrit par un spécialiste et sa surveillance est assurée par le médecin généraliste en collaboration avec un laboratoire d'analyse médicale. Les AVK sont caractérisés par une fenêtre thérapeutique étroite et l'équilibre thérapeutique s'avère parfois difficile à obtenir. Pour chaque patient, la dose nécessaire d'AVK est variable et doit être ajustée selon les résultats de l'INR. Les interactions médicamenteuses, les habitudes alimentaires et les maladies intercurrentes doivent être clairement identifiés et suivies dans le temps, afin d'ajuster le traitement AVK en conséquence [262].

Les AVK entrainent un risque accru de saignement majeur. Celui-ci est estimé à $3 \%$ par an. Ces hémorragies viennent au $1^{\text {er }}$ rang des accidents iatrogènes, avec $13 \%$ des hospitalisations pour effets indésirables médicamenteux, soit environ 17000 hospitalisations par an. Elles sont directement responsables de $0,6 \%$ des décès par an recensés chez les patients traités au long cours par AVK [122].

\subsection{Les antivitamines $\mathrm{K}$}

La découverte de la première molécule AVK date de 1920, c'est le dicoumarol, principe actif des raticides ou « mort aux rats ». Il a fallu attendre les années 40 pour définir la dose thérapeutique.

L'action anticoagulante des AVK est une action indirecte, elle est liée à la réduction de la synthèse hépatique des facteurs de coagulation vitamines K-dépendants (facteurs II, VII, IX et $X)$. Le degré d'anticoagulation obtenue est dépendant de la dose prescrite et de la susceptibilité individuelle. Il est apprécié par la valeur du temps de Quick exprimé en International Normalized Ratio (INR). La sensibilité du malade au médicament étant imprévisible, la posologie doit être adaptée et contrôlée régulièrement en fonction de la valeur de l'INR [125].

Les valeurs ciblées de l'INR se situent habituellement entre 2 et 3 lorsque le risque thrombotique est modéré et entre 3 et 4,5 lorsqu'il est majeur [12]. 
Il existe deux familles d'AVK : les indanediones et les coumariniques. Trois molécules sont disponibles en France : l'acénocoumarol, la warfarine et la fluindione $[9,10]$.

\subsubsection{Acénocoumarol}

L'acénocoumarol est un dérivé coumarinique à demi-vie courte (8 à 9 heures), commercialisé depuis 1990 sous le nom de MINISINTROM ${ }^{\circledR}$ à la dose de $1 \mathrm{mg}$ et de SINTROM $^{\circledR}$ à la dose de $4 \mathrm{mg}$ sous forme de comprimé quadrisécable [130]. C'est un AVK d'action rapide et de courte durée d'action (24 heures). Ce médicament est administré en 1 à 2 prises par jour. En cas de prise unique, il est préférable que la prise ait lieu le soir, afin de modifier la posologie dès que possible après les résultats de l'INR. L'utilisation de cet AVK est cependant peu prescrite car plus à risque d'instabilité thérapeutique [262].

\subsubsection{Warfarine}

La warfarine est l'AVK le plus prescrit dans le monde. Elle est commercialisée, en France depuis 1977, sous le nom de COUMADINE ${ }^{\circledR}$ à la dose de $2 \mathrm{mg}$ et $5 \mathrm{mg}$, à raison de 1 prise par jour [147]. Il s'agit d'un dérivé coumarinique à demi-vie longue (35 à 45 heures). L'effet anticoagulant est maximal entre 72 et 96 heures suivant l'administration. La durée d'action d'une seule dose de warfarine varie selon les individus entre 2 à 5 jours (96 à 120 heures) [262].

\subsubsection{Fluindione}

La fluindione est le seul dérivé indanedione. Elle est prescrite depuis 1988 sous le nom de PREVISCAN ${ }^{\circledR}$ à la dose de $20 \mathrm{mg}$ en comprimé quadrisécable [144]. C'est l'AVK le plus utilisé en France. Il s'agit d'une molécule à demi-vie longue (30 heures) avec un délai d'action et une durée d'action prolongés (48 à 72 heures) [262].

\subsection{Arrêt ou maintien du traitement par AVK ?}

Près de $10 \%$ des patients sous AVK ont chaque année d'une intervention chirurgicale [9]. Trois attitudes cliniques sont possibles: maintenir le traitement, l'arrêter ou réaliser un relais héparinique. Le choix de la modalité vis-à-vis de la gestion des AVK en cas de chirurgie orale dépend en premier lieu du type de chirurgie envisagée (chirurgie dento-alvéolaire ou chirurgie à risque haut hémorragique) [37]. Le deuxième critère est la présence ou non de facteurs locaux ou généraux susceptibles de majorer le risque hémorragique. L'existence de plusieurs facteurs de risque peut conduire à une situation à haut risque hémorragique. Dans les rares cas de chirurgie orale à risque hémorragique élevé, deux situations peuvent être distinguées en fonction du risque thrombotique (voir annexe 8). Enfin, le cas particulier de l'association AVK + AAP sera également abordé.

\subsubsection{Anesthésie locale (AL) et loco-régionale (ALR)}

Bajkin et coll., 2012 [34] rapporte l'inocuité de la réalisation d'une anesthésie locale chez des patients sous AVK quelle que soit la technique pratiquée : infiltration sous-muqueuse, bloc du nerf alvéolo-dentaire (BNAI). Sur un total de 560 injections, dont 96 BNAI, seuls deux hématomes mineurs sont décrits. Aucun hématome expansif dans l'espace ptérygomandibulaire n'est signalé.

\subsubsection{Chirurgie dento-alvéolaire}

\subsubsection{Extraction(s) dentaire(s)}

Wahl, 1998 [283] dans une revue systématique rapporte que sur 493 patients ayant arrêté leur AVK avant une procédure dentaire. Cinq patients $(1 \%)$ ont présenté des complications thrombotiques sévères dont 4 fatales. Akopov et coll., 2005 [14] dans une étude rétrospective sur une période de 12 mois rapportent que sur 197 AVC ischémiques d'origine cardiaque, 14 patients (soit un taux de 7,1\%) avaient interrompu leur traitement par warfarine 5,4 jours en moyenne avant l'accident cérébral en vue d'une intervention chirurgicale programmée. L'instauration d'un relais préalable ou non n'est pas précisé dans cette étude.

L'arrêt d'un traitement AVK comporte donc un risque d'accident thrombotique non négligeable et inacceptable dans le cadre d'une chirurgie à faible risque hémorragique. Deux études comparatives, randomisées et ouvertes $[55,99]$ ont également montré que le taux de saignement postopératoire après extraction dentaire n'était pas statistiquement différent entre le groupe de patient pour lequel la warfarine a été maintenue du groupe où elle é été arrêtée deux jours avant la procédure lorsqu'une hémostase chirurgicale était réalisée.

Karsh et coll., 2011 [180] ont comparé la perte sanguine après extraction dentaire en fonction du mode de gestion de l'AVK (poursuite, arrêt, substitution) chez des patients porteurs de prothèses valvulaires (avec un INR cible entre 3 et 4). La perte sanguine a été déterminée en mesurant la quantité de sang imbibée au niveau de compresses pendant les 20 premières minutes. La quantité de saignement a été de $2486 \pm$ $1408 \mathrm{mg}$ (warfarine seule), $999 \pm 425 \mathrm{mg}$ (relais warfarine HPBM), $1288 \pm 982 \mathrm{mg}$ (relais warfarine - HNF) et $1726 \pm$ $876 \mathrm{mg}$ (contrôle). Une hémotase chirurgicale a été ensuite réalisée après les 20 minutes de compression à l'aide d'une mèche d'oxycellulose et des points de sutures. Aucun saignement grave n'est rapporté et le nombre de compresse(s) utilisée(s) ne diffère pas selon les groupes. Les résultats de cette étude montrent que malgré une perte sanguine plus importante en cas de maintien du traitement par warfarine, celle-ci est de faible intensité et aisément contrôlable par des mesures d'hémostase locale. 
Les études, présentées sous forme de tableau synthétique en annexe 7, confirment qu'il n'existe aucun élément en faveur de l'arrêt des AVK avant des extractions dentaires.

L'utilité d'une adaptation posologique ou d'un traitement substitutif (relais héparinique) en cas d'extraction(s) dentaire(s) et ce qu'elle que soit la valeur de l'INR et/ou du risque thrombo-embolique comparativement à la stratégie du maintien de l'AVK n'a pu être démontrée [23, 33, 39, 46, 66, 73, $82,104,114,115,174,183,229,244,247,254,269]$.

Quel que soit le risque thrombo-embolique du patient, en cas de chirurgie dento-alvéolaire, le traitement par AVK ne doit pas être suspendu et la mise en place d'un relais héparinique ne doit plus être pratiqué.

Sur les 28 études contrôlées retenues [17, 20, 21, 28, 33, $41,42,45,46,51,55,57,58,63,82,99,114,126,181,209$, $223,225,249,254,255,263,269,290]$ ayant évalué la poursuite du traitement AVK en cas d'extraction dentaires, les facteurs importants permettant d'assurer une sécurité hémorragique acceptable sont une valeur pré-opératoire de l'INR stable et inférieur à 4 et le recours systématique à une hémostase locale.

En cas de poursuite du traitement par AVK avec un INR stable et inférieur ou égal à 4, le risque de complications hémorragiques sévères après extraction(s) dentaire(s) (recours à l'hopital et reprise chirurgicale) varie entre 0 et 2,77 \% selon ces études. Aucune complication grave (tranfusion, décès) n'est rapportée.

Une monothérapie antiplaquettaire (le plus souvent de l'aspirine ou du clopidogrel) associé au traitement AVK n'expose pas un surcoût d'événement de saignement [35, 223, 225].

Cette attitude clinique d'intervenir sans modification préopératoire du traitement AVK est la plus sûre pour le patient, mais également la moins onéreuse en comparaison au coût d'un relai héparinique $[66,73,242]$.

\subsubsection{Implant(s) dentaire(s)}

La pose d'implant(s) dentaire(s) chez les patients sous AVK est envisageable sauf risque médical associé [205]. Elle est contre-indiquée chez les patients à haut risque d'endocardite infectieuse, en particulier les patients porteurs d'une prothèse valvulaire mécanique ou biologique [5].

Dans une étude cas - témoin, Bacci et coll., 2011 [28] ont comparé l'effet de la prise de warfarine avec un INR $<4$ (1 heure avant la chirurgie) sur la survenue de complication hémorragique post-opératoire après la pose d'implant(s) dentaire(s). La même procédure d'hémostase chirurgicale associant sutures + pression sur une compresse imbibée d'acide tranexamique pendant 10 minutes est appliquée dans les deux groupes. Deux complications de saignements pour 50 patients sont rapportées dans le groupe traité (maintien de l' AVK) et trois complications pour 109 patients dans le groupe témoin (patients en bonne santé ne prenant pas d'AVK). Toutes les complications hémorragiques ont été traitées avec succès par une simple pression mécanique avec de l'acide tranexamique. Aucune hémorragie grave n'est signalée.

\subsubsection{Chirurgie à risque hémorragique élevé}

En cas de chirurgie à haut risque hémorragique, l'objectif est de limiter le risque de saignement et/ou d'hématome compressif lié à l'acte. L'arrêt du traitement par AVK parait alors offrir la meilleure protection contre la survenue d'une hémorragie postopératoire, mais cette gestion des AVK augmente le risque de survenue d'événement thrombotique durant la période postopératoire. Dès lors, le risque thrombotique lié à l'arrêt momentané de l'AVK doit être pris en compte. Son évaluation est de la responsabilité du médecin qui a prescrit l'AVK.

Les patients sous anticoagulants au long cours peuvent être classés en fonction du risque thrombo-embolique en risque élevé, modéré ou faible [39]. Les patients à haut risque ont un risque thrombotique artériel annuel de $10 \%$ ou plus (ex : patient porteur de valve mécanique, syndrome coronarien récent $<6$ mois) et un risque thrombo-embolique veineux à 1 mois à plus de $10 \%$ (ex : thrombose veineuse $<6$ mois). Un risque thrombo-embolique est considéré comme faible pour les patients présentant un risque thrombotique artériel annuel de moins de $5 \%$ (ex : ACFA) et un risque thrombotique veineux de moins de $2 \%$ (thrombose veineuse $>6$ mois) $[23,39]$. Douketis et coll., 2012 [86] rapportent dans une revue systématique, que le taux moyen thrombotique en péri-opératoire chez les patients sous AVK était estimé à $0,60 \%$ chez les patients ayant une MTEV, de $0,57 \%$ chez les patients en ACFA et de $0,80 \%$ chez les patients porteurs de valves mécaniques avec une gravité plus élevée. Il est établi que le taux de récidives thrombotiques suite à l'arrêt inopiné et/ou dans un contexte chirurgical d'un traitement par AVK est plus important lors du premier mois et est plus élevé pour les patients chez lesquels le risque thrombogène est élevé [264]. Aussi, deux situations peuvent être distinguées : les patients à risque thrombotique faible et les patients à risque thrombotique élevé $[9,122]$.

Dans les cas de chirurgie orale à risque hémorragique élevé (ex : chirurgie pré-implantaire), le médecin prescripteur doit être informé afin d'évaluer le risque thrombotique, de définir la stratégie thérapeutique vis-à-vis du traitement par AVK (arrêt pur et simple des AVK, relais héparinique) et des modalités de prise en charge (en pratique de ville, prise en charge hospitalière) [172].

\subsubsection{Patients à risque thrombotique faible}

Chez les patients traités pour une ACFA sans antécédent embolique et les patients traités pour une MTEV à risque 
modéré, le risque thrombotique lié à un cas un arrêt de l'AVK est considéré comme suffisamment faible (que ce soit en terme de fréquence ou de gravité), pour proposer d'interrompre l'anticoagulation par AVK sans relais pré-opératoire par héparine $[85,86,110,122]$. L'arrêt de l'AVK en pré-opératoire en cas de chirurgie à risque hémorragique élevé chez un patient à risque thrombotique faible est la stratégie thérapeutique recommandée par le GEHT et la HAS [122]. Un schéma récapitulatif du protocole d'arrêt des AVK est présentée en annexe 9. L'AVK doit être stoppé 3 à 5 jours avant la date de l'intervention. L'AVK doit être repris dans les 24 à 48 heures postopératoires. La vitesse d'obtention d'un nouvel état d'équilibre est comprise entre 4 et 5 demi-vies après toute modification de posologie [262]. Lorsque la reprise des AVK n'est pas possible dans ce délai de 48 heures, un relais postopératoire par HPBM ou HNF à dose curative doit être réalisé [122].

\subsubsection{Patients à risque thrombotique élevé}

Les patients porteurs de valves cardiaques mécaniques (tout type), les patients traités pour une ACFA avec antécédent embolique et les patients traités pour une MTEV à haut risque (TVP proximale et/ou EP $<3$ mois, MTEV récidivante idiopathique), le risque de complications thrombotiques est considéré comme majeur [122]. Pour réduire la fenêtre thérapeutique, c'est-à-dire la durée de la période durant laquelle le patient est insuffisamment anticoagulé (valeur INR < INR cible), un relais péri-opératoire par héparine à doses curatives est recommandé [122]. Le recours à un traitement substitutif vise également à mieux maîtriser le saignement opératoire, tout en minimisant le risque thrombo-embolique [33, 84, 108]. Trois options sont possibles : héparine non fractionnée (HNF) à la séringue électrique, HNF en sous-cutanée (2 à 3 injections par jour), héparines de bas poids moléculaires (HBPM) en souscutanée (1 à 2 injections par jour).

Les modalités d'un relais héparinique pré-opératoire en vue d'une chirurgie sont détaillées dans les recommdandations émises par le GEHT et l'HAS publiées en 2008 [122]. En premier lieu, il est recommandé de mesurer l'INR 7 à 10 jours avant l'intervention. Si l'INR est en zone thérapeutique, il est recommandé d'arrêter les AVK 4 à 5 jours avant l'intervention. La valeur de 1,5 est retenue comme seuil d'INR en dessous duquel il n'y a pas de majoration des complications hémorragiques péri-opératoires. Chez le patient à haut risque thrombo-embolique, le relais par héparine consiste à couvrir la période préet postopératoire durant laquelle les AVK ne sont pas efficaces. Pour cela, il faut commencer l'héparine à doses curatives 48 heures après la dernière prise de fluindione ou de warfarine ou 24 heures après la dernière prise d'acénocoumarol. La réalisation d'un INR, la veille de l'intervention, est recommandée. Les patients ayant un INR supérieur à 1,5 la veille de l'intervention recevront $5 \mathrm{mg}$ de vitamine $\mathrm{K}$ per os et un nouveau contrôle de l'INR devra être réalisé le matin de l'intervention.
L'arrêt des héparines est recommandé comme suit : HNF IV à la seringue électrique (arrêt 4 à 6 heures avant la chirurgie), HNF SC (arrêt 8 à 12 heures avant la chirurgie), HBPM (dernière dose 24 heures avant l'intervention). Le contrôle du TCA ou de l'activité anti-Xa le matin de l'intervention n'est pas nécessaire. Après l'intervention, les AVK et les héparines sont repris dès que possible en fonction du risque hémorragique et thrombo-embolique. Le traitement par héparine est interrompu après 2 INR successifs en zone thérapeutique à 24 heures d'intervalle. Un schéma récapitutatif du protocole d'arrêt et de reprise de l'AVK avec un relais héparinique en péri-opératoire est présentée en annexe 10 . Si la procédure de relais n'est pas réalisée dans un parcours de soins coordonné en ville, la HAS recommande d'hospitaliser le patient, au plus tard la veille de la chirurgie, pour adapter l'anticoagulation.

La mise en place d'un relais héparinique permet globalement de réduire le taux d'évènement thrombotique mais augmente le taux d'évènement hémorragique (comparativement au protocole d'arrêt des AVK) [272, 273, 274]. Kovacs et coll., 2004 [84] dans une étude prospective, multicentrique chez des patients à haut risque thrombo-embolique (prothèse valvulaire mécanique et ACFA avec facteurs de risque majeur) ont mesuré l'efficacité et le profil de sécurité en cas de relais héparinique à dose curative par une HBPM (dalteparine $200 \mathrm{U} / \mathrm{kg} / \mathrm{jour}$ ) en ambulatoire. Dans cette étude, la fréquence de thrombose et de saignement sévère était de 3,6 et 6,7 \% respectivement.

En résumé, en cas de chirurgie dento-alvéolaire, le risque hémorra-
gique est faible et facilement contrôlable par une hémostase locale.
Les extractions dentaires et la pose d'implants dentaires peuvent
être réalisées sans interrompre les AVK (après contrôle de l'INR et
vérification d'une valeur $\leq 4$ ).
Pour les interventions et actes invasifs à risque hémorragique
élevé, l'arrêt des AVK avec ou sans relais héparinique, en fonction
du risque thrombotique du patient, est recommandé. La décision
d'interrompre temporairement le traitement AVK, doit être prise
après concertation avec le médecin qui a prescrit l'AVK, l'anesthé-
siste (en cas d'anesthésie générale) et le chirurgien. L'ensemble de
la démarche adoptée doit faire l'objet d'un protocole écrit.

\subsection{Quel test biologique doit-on utiliser pour évaluer le risque hémorragique chirurgical ?}

L'examen biologique permettant d'estimer le risque hémorragique chirurgical d'un patient sous AVK est l'international normalized ratio (INR) $[49,125]$. Une valeur de l'INR supérieure à 6 est associée à une augmentation significative du risque d'hémorragie majeure spontanée [59]. Les patients ayant un INR cible élevé (au maximum entre 3,5 et 4,5 ) ont un risque d'hémorragie (mineure, majeure) spontanée plus élevé que ceux ayant un INR cible entre 2 et 3 [9, 10]. En revanche, aucune corrélation n'a été trouvée entre le risque hémorragique et la molécule AVK prescrite ou la durée écoulée depuis le début du traitement [59]. 
- En cas de chirurgie dento-alvéolaire, 28 études observationnelles regroupant 2150 patients traités par un AVK dans les zones thérapeutiques usuelles (INR compris entre 2 et 3 et entre 3 et 4 ), montrent que les extractions dentaires simples ou chirurgicales peuvent être réalisées sans interrompre les AVK.

La valeur de 4 peut être retenue comme seuil d'INR en dessous duquel il n'y a pas de majoration des complications hémorragiques péri-opératoires. Cette évaluation biologique préopératoire permet de vérifier l'absence d'un éventuel surdosage en $A V K$, facteur de risque hémorragique majeur en cas de chirurgie. Le contrôle de l'INR doit être effectué dans les 24 heures qui précèdent l'intervention chirurgicale. En cas de surdosage (INR supérieur ou égal à 4), l'intervention chirurgicale doit être reportée et le médecin prescripteur informé. Des mesures correctives doivent être instaurées sans délai par le médecin prescripteur afin de ramener l'INR en zone thérapeutique (saut d'une prise, apport de vitamine $\mathrm{K}$, hospitalisation en urgence) [122]. En zone thérapeutique, la majorité des études observationnelles montrent l'absence de corrélation positive entre la valeur pré-opératoire de l'INR et le taux d'incidence de saignement postextractionnel [42, 45, 74, 223, 255]. Une seule étude identifie la valeur pré-opératoire de l'INR comme facteur de risque de saignement postopératoire [173]. En revanche, dans toutes ces études $[42,45,74,173,223$, 255], l'inflammation gingivale et/ou périapicale a été identifiée comme un facteur majeur du sur-risque hémorragique chez les patients sous AVK.

L'autre condition est d'avoir un INR stable. Il est donc nécessaire de connaitre les valeurs des derniers bilans d'INR ou le carnet de suivi. Dans le cadre de la surveillance de son traitement anticoagulant, les patients réalisent habituellement un examen INR toutes les 2 à 4 semaines. Un INR instable est considéré comme un facteur de gravité et le médecin qui a prescrit l'AVK doit être contacté. Il est recommandé de différer la chirurgie à 1 mois. Si la chirurgie ne peut être différée, le choix d'un relais héparinique et/ou une prise en charge hospitalière est à discuter avec le médecin prescripteur [172].

Cependant, prudence est de garder cette valeur seuil en présence de comorbidités [168]. Dans une grande série de cas prospectif $(n=500)$, Cocero et coll., 2014 [63] préconisent des valeurs plus basses avec des valeurs limites de 2,8 chez les patients porteurs d'une prothèse valvulaire mécanique et de 2,3 pour les patients diabétiques, les insuffisants hépatiques et rénaux.

- En cas de chirurgie à haut risque hémorragique, l'interruption des AVK est nécessaire. Une concertation avec le médecin qui a prescrit l'AVK est impérative afin d'évaluer le risque thrombotique associé à l'arrêt de l'AVK. Chez les patients à risque thrombotique faible, un arrêt des AVK sans traitement substitutif peut être proposé. Chez les patients à haut risque thrombotique, un relais pré- et post-opératoire avec une héparine est recommandé. Dans les deux cas, la réalisation d'un INR la veille de l'intervention est impérative. Un seuil de sécurité hémostatique est défini avec une valeur de l'INR inférieure ou égale à 1,5 [122].

\subsection{Quelle hémostase locale doit-on réaliser pour contrôler le risque hémorragique chirurgical ?}

Au total, 26 études observationnelles prospectives randomisées $[17,19,20,28,33,41,42,45,46,51,55,57,58,82,99$, $114,126,181,209,223,249,254,255,263,269,290]$, présentées dans l'annexe 7, ont évalué l'intérêt de l'utiliser des hémostatiques locaux spécifiquement en chirugie dento-alvéolaire.

\section{- Sutures}

Une seule étude propective randomisée ouverte a mesuré l'impact de réaliser ou non des sutures sur l'incidence du saignement postopératoire en cas d'extraction(s) dentaire(s) chez des patients sous warfarine avec un INR $\leq 3$ [21]. De façon inattendue, les patients bénéficiant de sutures ont montré un taux plus élevé de saignement postopératoire. Ces résultats, bien que non significatifs, montrent que la réalisation de sutures, dans le but de diminuer le taux de saignement postopératoire n'est pas démontré en cas d'extraction simple et/ou l'absence de décollement gingival.

\section{- Hémostatiques chirurgicaux}

Huit études observationnelles ou comparatives [28, 33, 42, $55,82,254,255,223$ ] ont montré que l'utilisation d'éponge de gélatine, de collagène ou de gaze d'oxycellulose en tamponement intra-alvéolaire en complément des mesures conventionnelles (sutures + compression) permettait de prévenir efficacement le risque de saignement postopératoire postextractionnel chez les patients avec un INR $\leq 4$. En revanche, il n'existe aucune étude comparative entre ces différents hémostatiques chirurgicaux.

\section{- Colle de fibrine}

Trois études propectives randomisées en ouvert [41, 57, 126] ont évalué l'utilisation d'une colle de fibrine (BLERI$\mathrm{PLAST}^{\circledR}, \mathrm{FIBRIJET}^{\circledR}$ ) en adjuvant à une éponge de gélatine, collagène ou à une mèche d'oxycellulose et à des sutures. Les comparateurs étaient ces mêmes hémostatiques chirurgicaux employés seuls sans colle de fibrine. Aucune réduction significative du nombre d'événement de saignement postopératoire avec l'emploi de colle de fibrine n'est rapporté. A ce jour, l'intérêt de l'emploi de colle de fibrine, en prévention du saignement postopératoire, en raison de son coût et de sa dispensation restreinte (milieu hospitalier, traçabilité) n'est pas démontré chez les patients sous AVK en cas d'extraction(s) dentaire(s).

Bien que son utilisation en cas de reprise chirurgicale soit largement décrite dans les études observationnelles, son efficacité n'a jamais été évaluée, ni comparée avec d'autres hémostatiques chirurgicaux.

\section{- Colle cyanoacrylate}

Deux études observationnelles $[115,193]$ ont donné des résultats favorables sur l'efficacité d'une membrane de cellulose 
enduite d'une colle cyanoacrylate dans la prévention du risque de saignement postéopératoire chez des patients sous AVK après extraction(s) dentaire(s). Dans une autre étude [17] prospective, concernant 30 patients pour extractions simples, cette approche a été comparée au tamponnement intra-alvéolaire à l'aide d'éponge de gélatine. Le nombre d'évènement de saignement postopératoire dans le groupe traité (colle cyanoacrylate) est significativement diminué compartivement au groupe contrôle (éponge de gélatine). Du fait du faible niveau preuve de ces trois études, il est difficile de privilégier cette approche par rapport aux autres mesures d'hémostase locale.

\section{- Acide tranexamique}

Trois études comparatives [46, 249, 263] versus placebo, randomisées, en double aveugle ont démontré l'efficacité de l'acide tranexamique en topique (irrigation intra-alvéolaire puis en bain de bouche 4 fois/jour pendant 5 à 7 jours) dans la prévention du risque de saignement en cas d'extraction(s) dentaire(s) et de maintien du traitement par AVK avec un INR $<4$.

Une étude comparative [58] (5 jours versus 2 jours), randomisée, en double aveugle, a montré que l'acide tranexamique en bain de bouche ( 4 fois par jour) pendant 2 jours est aussi efficace que 5 jours dans le contrôle du saignement postopératoire chez les patients sous AVK.

Une étude observationnelle [29] cas-témoin (maintien AVK versus pas d'anticoagulant), a évalué l'efficacité d'une compression avec une compresse imbibée d'acide tranexamique en complément de suture dans le contrôle de l'hémostase après la pose d'implant(s) dentaire(s). Le taux de saignement postopératoire est faible dans les deux groupes $(<5 \%)$. Tous les saignements ont été contrôlés par un simple renouvellement de la procédure (compression mécanique imbibée d'acide tranexamique).

Au total, au vu des données de la littérature, le recours à des hémostatiques locaux (acide tranexamique, éponge de gélatine, collagène, mèche d'oxycellulose) en cas d'extraction(s) dentaire(s) chez des patients sous AVK doit être systématique. En l'état actuel des connaissances, le groupe de travail n'a pas pu priviligier un produit ou une classe d'hémostatique chirurgical. Dans un contexte préventif, la pertinence de l'utilisation des colles biologiques reste à démontrer.

En cas de chirurgie à risque hémorragique élevé, et en l'absence de possibilité d'éffectuer une compression mécanique ou d'une localisation critique, il est impératif de réaliser le geste en situation de sécurité hémostatique avec un contrôle pré-opératoire de l'INR $\leq 1,5$. En cas de risque thrombotique élevé, un relais héparinique est nécessaire.

\subsection{Quelles sont les prescriptions médicamenteuses potentialisatrices du risque hémorragique ?}

Un grand nombre de médicaments interfèrent avec les AVK. Certains concourent à la survenue de surdosage biologique
(INR $\geq 4,0)$. De nombreuses molécules prescrites par les professionnels de la cavité orale sont potentialisatrices du risque hémorragique des AVK et peuvent justifier d'une surveillance transitoirement accrue de l'INR.

\section{- Prescription antibiotique}

Toute prise d'antibiotique est un facteur risque de déséquilibre de la flore digestive et de perturbation de synthèse endogène de vitamine $K$, avec par voie de conséquence, un déséquibre du traitement par AVK et une majoration du risque hémorargique [187, 289]. Les macrolides (à l'exception de la spiramycine), la doxycycline et le métronidazole sont déconseillés [282]. Concernant la prescription d'amoxicilline seule ou en association avec l'acide clavulanique, des données contradictoires sont rapportées dans la littérature. Des cas cliniques [38, 52, 79, 188, 268], une étude cas-témoin [241], une enquête prospective [261] rapportent la survenue de surdosages et d'accidents hémorragiques suite à l'introduction $d^{\prime}$ amoxicilline ou d'amoxicilline + acide clavulanique chez des patients traités par AVK et initialement parfaitement équilibrés. A l'inverse, dans une étude en double aveugle, randomisée, versus placebo, mais en l'absence d'inflammation et/ou d'infection, la prescription d'amoxicilline 1g, 2 fois par jour, pendant 7 jours + acide clavulanique ne modifie pas significativement la valeur de l'INR [292]. Il est à rappeler qu'une infection avec les modications métaboliques qui l'accompagnent est en soit un facteur de risque de déséquilibre du traitement anticoagulant oral. Aussi il est difficile en cas d'infection, de distinguer la part liée à la réaction inflammatoire de celle liée au médicament [261]. Dans tous les cas, une infection bucco-dentaire avérée se doit d'être traitée chez ces patients avec les antibiotiques usuels adéquats avec un contrôle rapproché de l'INR [159]. À noter qu'une antibioprophylaxie ne modifie pas la valeur de l'INR [208].

\section{- Prescription antifongique}

Le miconazole est strictement contre-indiqué quel que soit son mode d'administration, y compris par voie topique [282] Tous les antifongiques azolés sont déconseillés [282]. Chez les patients traités par AVK, l'amphotéricine B est l'antifongique recommandé pour le traitement des candidoses oropharyngées.

\section{- Prescription antalgique}

Pour les douleurs faibles à modérées, la prescription d'aspirine (à doses antalgiques) est formellement contre-indiquée et celles des AINS fortement déconseillées en raison du risque d'hémorragies systémiques sévères imprévisibles (hémorragies digestives et intracrâniennes) [282]. La prise de paracétamol est possible mais fait l'objet de précautions d'emploi en cas de prescription aux doses maximales ( $4 \mathrm{~g}$ par jour) pendant au moins 4 jours [282]. Des études rapportent une augmentation significative de l'INR suite à la prescription de paracétamol à doses thérapeutiques (2 à $4 \mathrm{~g} /$ jour) [171, 200, 206, 291]. Chez le sujet âgé, une adaptation posologique $(<2 \mathrm{~g} /$ jour $)$ et la prescription d'un INR durant la période postopératoire sont des 
mesures permettant de limiter et de détecter la survenue de tout surdosage [170]. Pour les douleurs de modérées à sévères, les dérivés opiacés (codéine, tramadol) peuvent être prescrits, le tramadol faisant l'objet néanmoins d'une précaution d'emploi [282].

\section{- Prescription anti-inflammatoire}

Si une prescription anti-inflammatoire se révèle nécessaire, la prescription de corticoïde est à privilégier.

\subsection{Traitement curatif des complications hémorragiques}

Leurs prises en charge reposent toujours en premier lieu sur une reprise chirurgicale et sur la recherche d'une cause locale du saignement. L'absence de contrôle durable de l'hémorragie par les mesures usuelles d'hémostase locale est considérée comme un critère de gravité et une prise en charge hospitalière est alors nécessaire.

A l'admission du patient, une mesure de l'INR en urgence sera réalisée. Quelle que soit la valeur de l'INR, une reprise chirurgicale doit être pratiquée d'emblée. L'utilisation de colle biologique est cliniquement pertinente. En cas de surdosage, une antagonisation rapide à l'aide de vitamine $\mathrm{K}$ ou de concentrés de complexes prothrombiniques (CCP) peut être indiquée. En complément d'information, se rapporter aux recommandations du GEHT d'avril 2008 [122] concernant « la prise en charge des surdosages en antivitamines $K$, des situations à risque hémorragique et des accidents hémorragiques chez les patients traités par antivitamines $\mathrm{K}$ en ville et en milieu hospitalier » disponible sur www.has-sante.fr.

\section{Spécificités de la prise en charge des patients traités par anticoagulants oraux directs}

\subsection{Les enjeux}

Depuis 2009, une nouvelle classe d'anticoagulants oraux est disponible. Il s'agit d'anticoagulants oraux directs (AOD) sélectifs soit de la thrombine (anti-IIa) soit du facteur $X$ activé (anti-Xa) [12].

Leurs indications, limitées dans un premier temps, à la prévention du risque de la MTEV après une chirurgie orthopédique (prothèses totales de hanche et de genou) $[80,148,149]$ ont été élargies à partir de 2012 à la prévention des accidents thrombo-emboliques, chez les patients présentant une ACFA non valvulaire associée à un ou plusieurs facteurs de risque [7, $9,10,148,149,152]$. Les AOD sont contre-indiqués chez les patients atteints de rétrécissement mitral ou porteurs de prothèse valvulaire.

Les AOD sont principalement utilisés en alternative des AVK et/ou des HBPM et sont destinés à une large population. Ils ont modifié un certain nombre de protocoles. Le traitement préventif de la MTEV comprend une héparine par voie SC pendant 10 à 35 jours (traitement de référence actuel). De la même manière, le traitement curatif fait appel à une héparine pendant au moins 5 jours relayée par un AVK. Ces schémas thérapeutiques classiques, largement validés par de nombreux essais cliniques, présentent néanmoins des inconvénients: la nécessité d'examens biologiques rapprochés, le recours à une administration parentérale et une période de chevauchement de deux anticoagulants en cas de relais qui expose à un risque hémorragique accru. L'utilisation immédiate d'un AOD sans passer par une phase d'anticoagulation parentérale initiale rend cette modalité de traitement très pratique. Dans le traitement de l'ACFA non valvulaire, les AOD auraient des avantages siginificatifs sur les AVK (traitement de référence actuel) : l'absence d'interaction alimentaire, un nombre d'interactions médicamenteuses limité rendent compte d'une activité anticoagulante «plus prédictible » permettant de les administrer avec une dose fixe (pour chaque patient) et de ne pas recourir à une surveillance biologique [7].

Cette avancée importante sur les AVK semble attrayante, mais l'utilisation des AOD fait aujourd'hui débat [15]. L'absence de surveillance biologique est à la fois un avantage et un inconvénient :

- avantage car la pratique régulière de l'INR et l'adaptation des doses en fonction des résultats est une contrainte pour les patients traités par AVK,

- inconvénient, car l'absence d'information de l'effet du médicament sur l'anticoagulation expose au risque éventuel de sous-dosage source d'inefficacité ou de surdosage entrainant un sur-risque hémorragique.

De nombreux accidents hémorragiques ont été rapportés. Sous AOD, le risque d'hémorragies intra-crâniennes serait moins élevé que sous warfarine. A l'inverse, celui des hémorragies gastro-intestinales serait majoré. De plus, il n'existe pas d'antidote en cas de surdosage et leur coût est bien supérieur aux traitements classiques [10]. Leur utilisation est actuellement étroitement surveillée tant au niveau national par l'Agence nationale de sécurité du médicament et des produits de santé (Ansm) qu'au niveau européen par l'Agence européenne des médicaments (EMA) $[10,11]$. Depuis leur commercialisation en 2009, la prescription de ces AOD est associée à un pourcentage élevé de mésusage (non respect des indications et/ou des contre-indications) ayant conduit à des situations à risque hémorragique majoré ou inapproprié [11]. L'absence de test biologique fiable en routine permettant d'estimer le risque hémorragique et l'absence d'antidote spécifique rendent délicates la gestion de ces AOD en cas de chirurgie et d'accidents hémorragiques.

En 2013, en France, 265000 patients ont été traités par AOD [10].

\subsection{Les anticoagulants oraux directs}

Trois molécules AOD sont actuellement commercialisées en France : le dabigatran étexilate, qui inhibe le facteur II activé 
(IIa ou thrombine) ; le rivaroxaban et l'apixaban qui inhibent le facteur $X$ activé $(X a)[9,10]$.

\subsubsection{Dabigatran étexilate}

Le dabigatran étexilate est un inhibiteur direct de la thrombine (anti-IIa direct) dont l'inhibition est concentration dépendante, compétitive, hautement sélective et réversible [97, 279]. Ce médicament est commercialisé sous le nom de PRADAXA ${ }^{\circledR}$ et existe en trois dosages en France : $75 \mathrm{mg}, 110$ $\mathrm{mg}$ et $150 \mathrm{mg}$. PRADAXA ${ }^{\circledR} 75 \mathrm{mg}$ et PRADAXA ${ }^{\circledR} 110 \mathrm{mg}$ sont indiqués dans la prévention primaire des événements thromboemboliques veineux chez les adultes ayant bénéficié $d^{\prime} u n e$ chirurgie progammée pour prothèse totale de la hanche (PTH) ou du genou (PTG) (AMM obtenue en mars 2008) [148]. La durée maximum du traitement est limitée à 35 jours. PRADAXA ${ }^{\circledR}$ $110 \mathrm{mg}$ et PRADAXA ${ }^{\circledR} 150 \mathrm{mg}$ sont utilisés dans la prévention de l'AVC et de l'embolie systémique (ES) chez les patients adultes présentant une ACFA non valvulaire associée à un ou plusieurs des facteurs de risque (antécédent d'AVC/AIT, ES, FEV gauche $<40 \%$, insuffisance cardiaque symptomatique (classe NYHA $\geq$ II), âge $\geq 75$ ans, diabète, HTA) (AMM obtenue en août 2011). Les posologies de $220 \mathrm{mg}$ par jour $(2 \times 110 \mathrm{mg})$ et de $300 \mathrm{mg}$ par jour $(2 \times 150 \mathrm{mg})$ sont recommandées chez les patients où il existe un ou plusieur(s) facteur(s) de risque(s) $[148,282]$. Le dabigatran étexilate a été évalué et son efficacité comparée pour chacune de ses indications, soit à l'enoxaparine (40 mg $\times 1$ fois/jour) (essai RE-MODEL) [94], RE-NOVATE I [95] et II [98]), soit à la warfarine (avec un INR cible de 23) (études RECOVER [258] et RE-LY $[67,100])$. Au cours des études cliniques de phases I, II et III, des facteurs de risques d'hémorragies graves ont été identifiés, à savoir une posologie élevée (> $220 \mathrm{mg} / \mathrm{jour}$ ), une insuffisance rénale (clairance de la créatinine $<50 \mathrm{~mL} / \mathrm{min}$ ) et le sujet âgé (> 80 ans) $[67,128$, 258]. À noter, qu'il n'existe cependant aucune différence entre le dabigatran étexilate et la warfarine en terme de mortalité globale.

\subsubsection{Rivaroxaban}

Le rivaroxaban est un inhibiteur direct, hautement sélectif et compétitif du facteur $X a$ (anti-Xa direct). Sa sélectivité pour le facteur $X a$ est supérieure à 10000 fois celle des autres sérines protéases (facteurs $\mathrm{Va}, \mathrm{IXa}, \mathrm{XIa}$, thrombine et protéine $\mathrm{C}$ activée) [119]. Le rivaroxaban peut inhiber à la fois le facteur Xa libre mais également le facteur $X a$ au sein du complexe prothrombinase ainsi que le facteur $X a$ associé au caillot. L'inhibition du facteur $X a$ interrompt les voies intrinsèque et extrinsèque de la cascade de coagulation sanguine, inhibant ainsi la génération de thrombine et le développement du thrombus. Le rivaroxaban n'inhibe pas la thrombine (facteur IIa) et il n'a aucun effet démontré sur les plaquettes [217]. Le rivaroxaban est commercialisé sous le nom de XARELTO ${ }^{\circledR}$ et existe en France sous trois dosages : $10 \mathrm{mg}, 15 \mathrm{mg}$ et $20 \mathrm{mg}$. Les indications pour le rivaroxaban diffèrent en fonction du dosage utilisé. XARELTO ${ }^{\circledR} 10 \mathrm{mg}$ est indiqué pour la prévention primaire des évènements thrombo-emboliques veineux chez les patients bénéficiant d'une intervention programmée de chirurgie orthopédique majeure (PTH, PTG) (AMM obtenue en décembre 2008) [149]. La durée maximale du traitement est habituellement de 35 jours et démarre 6 à 10 heures après l'intervention. XARELTO ${ }^{\circledR} 15 \mathrm{mg}$ et XARELTO ${ }^{\circledR} 20 \mathrm{mg}$ sont recommandés dans la prévention des AVC et embolies systémiques en cas d'ACFA non valvulaire associée à un ou plusieurs facteurs de risque (idem à ceux cités pour le dabigatran) et le traitement préventif et curatif des TVP et de leurs complications (AMM obtenue en 2012) [282]. Les études RECORD 1 [96], 2 [178] et 3 [189] ont montré la supériorité du rivaroxaban (10 mg 1 fois/j) par rapport à l'enoxaparine (40 mg 1 fois/ j) dans la thrombophylaxie de la chirurgie orthopédique programmée pour une durée totale respectivement, entre 10 et 14 jours pour la PTG et entre 31 et 39 jours la PTH. Dans la prévention et le traitement de la MTEV (études EINSTEIN) [92, 93] et la prévention des AVC ischémiques et des ES chez les sujets atteints d'ACFA non valvulaire présentant un ou plusieurs facteurs de risque (étude ROCKET-AF) [238], le rivaroxaban a démontré sa non-infériorité en termes d'efficacité et de sécurité par rapport aux traitements classiques (énoxaparine, warfarine). Il n'y a pas de différence significative en ce qui concerne les hémorragies majeures et le taux de mortalité global. A noter que dans le groupe rivaroxaban, les hémorragies cérébrales mortelles étaient moins nombreuses.

\subsubsection{Apixaban}

L'apixaban, comme le rivaroxaban, est un inhibiteur direct et sélectif du facteur Xa, sans activité sur la thrombine [287]. C'est donc essentiellement sur leurs caractéristiques pharmacocinétiques, que ces deux molécules se distinguent. Il n'y a pas d'interaction alimentaire rapportée dans la littérature. Son élimination rénale est faible ( $25 \%$ sous forme active). De ce fait, l'altération de la fonction rénale modifie peu la pharmacocinétique de l'apixaban, contrairement aux autres AOD (rivaroxaban et surtout dabigatran étexilate) $[128,288]$. L'apixaban est commercialisé en France sous le nom d'ELIQUIS ${ }^{\circledR}$ et existe sous deux dosages : 2,5 et $5 \mathrm{mg}$. ELIQUIS ${ }^{\circledR} 2,5 \mathrm{mg}$ est indiqué dans thrombophylaxie en cas de PTH ou de PTG et ELIQUIS ${ }^{\circledR} 5 \mathrm{mg}$, dans la prévention des AVC ischémiques et des ES chez les patients atteints d'une ACFA présentant un ou plusieurs facteurs de risque (les mêmes que pour le dabigatran étixelate et le rivaroxaban) $[152,282]$. L'efficacité et la tolérance de l'apixaban ( $5 \mathrm{mg} \times 2$ fois/j) ont été évaluées dans chez les patients présentant une ACFA soit par rapport à l'aspirine (81 à 324 mg) (étude AVERROES) [68], soit à la warfarine (avec un INR cible 2-3) (étude ARISTOTLE) [120]. Ces deux études montrent des résultats globalement en faveur de l'apixaban. 


\subsection{Arrêt ou maintien du traitement par anticoagulant oral direct?}

Aucune étude n'est actuellement disponible pour évaluer le risque hémorragique en cas d'extraction dentaire chez un patient sous AOD. L'analyse exhaustive de la littérature n'a retrouvé que cinq sources d'information de faible niveau de preuve : un cas clinique [293], une série de cinq cas cliniques [47], trois revues systématiques de la littérature [77, 101, 105] et l'analyse rétrospective de l'essai RE-LY pour le dabigatran [164] et l'étude ROCKET-AF pour le rivaroxaban [238].

Romond et coll., 2013 [293] rapportent le cas d'un patient de 67 ans ayant pour antécédent une ACFA, un diabète de type 2, une HTA et une hyperlipidémie, traité par une bithérapie ayant bénéficié d'extractions dentaires multiples (8 dents maxillaires) associé à une alvéoloplastie et sous bithérapie dabigtran (2 $\times 150 \mathrm{mg} /$ jour $)+$ aspirine ( $81 \mathrm{mg} /$ jour $)$. Le dabigatran a été arrêté la veille au soir de l'intervention et repris le lendemain de l'intervention (fenêtre thérapeutique 24 à 48 heures). Une hémostase associant éponge de gélatine, sutures et compression a été réalisée suivie de la pose d'une prothèse amovible complète. Aucun saignement anormal n'est rapporté. Aucune complication thrombotique n'est signalée durant les sept mois postopératoire.

Breik et coll., 2014 [47] à partir d'une petite série de cas prospective $(n=5)$, conseillent de ne pas arrêter le dabigatran en cas d'extraction simple, le risque hémorragique pouvant être contrôlé par une hémostase locale. En revanche, les auteurs préconisent un arrêt du dabigatran en cas d'extractions multiples ou de procédure chirurgie orale complexe.

Davis et coll., 2013 [77] dans une revue systémique de la littérature préconisent de suivre la même attitude thérapeutique que celle adoptée avec la warfarine en cas d'extraction dentaire puisque toutes les études montrent que le risque hémorragique chez les patients traités par dabigatran est statistiquement similaire à celui observé chez les patients sous warfarine avec un INR entre 2 et 3.

Fahkri et coll., 2013 [101] dans une autre revue systématique de la littérature sont favorables à une fenêtre thérapeutique avec un arrêt des AOD 3 jours avant (si risque hémorragique élevé) ou 2 jours avant (si risque hémorragique modéré) avec une reprise des AOD 24 heures après l'intervention. En cas de risque de thrombotique élevé, un relais héparinique est proposé.

Pour Firriolo et Hupp, 2012 [105], il n'apparaît pas nécessaire d'interrompre la prise de dabigatran ou de rivaroxaban chez les patients présentant une fonction rénale normale et en l'absence de facteurs locaux ou systémiques pouvant majorer le risque hémorragique. L'arrêt des AOD est seulement préconisé en cas de saignement postopératoire non contrôlé par une hémostase locale. En cas de chirurgie orale ou maxillofaciale, à risque hémorragique élevé, les auteurs recommandent d'interrompre le dabigatran ou le rivaroxaban au moins
24 heures avant l'intervention chirurgicale ou plus en cas d'insuffisance rénale (clairance de la créatinine $<30-50 \mathrm{~mL} / \mathrm{min}$ ). Les $A 0 D$ devront être repris 24 à 48 heures après l'intervention.

Au cours de l'essai RE-LY [164], étude contrôlée randomisée en ouvert de non-infériorité de deux doses de dabigatran (110 mg $\times 2$ fois/jour; $150 \mathrm{mg} \times 2$ fois/jour) versus warfarine (avec un INR ciblé entre 2 et 3), chez plus de 18000 patients, 4591 sujets ont eu un geste invasif ou une chirurgie. Dix pour cent ont bénéficié d'une procédure dentaire. Il n'a pas été trouvé de différence entre les patients prenant du dabigatran et ceux sous warfarine concernant les saignements majeurs. Dans cette étude, les patients sous dabigatran dont la clairance de la créatinine était normale, étaient opérés entre 24 et 72 heures après la dernière prise (soit entre 2 et 5 demi-vies). Pour le rivaroxaban, les seules données disponibles sont issues de l'étude ROCKET-AF [238], étude contrôlée randomisée de « non-infériorité » versus warfarine à dose ajustée selon l'INR conduite chez 14264 patients traités au moins 18 mois dans l'indication de la prévention de l'AVC et de l'ES chez chez les patients en ACFA non valvulaire. Dans cette étude, le rivaroxaban était arrêté deux jours avant la procédure chirurgicale programmée (soit environ 4 demi-vies).

En dehors du champ strict de la chirurgie orale, des propositions et des suggestions ont été émises par le Groupe d'intérêt en hémostase périopératoire (GIHP) et le GEHT [259, 260]. Dans le cadre de la chirurgie programmée, la poursuite du traitement par $A O D$ est la règle en instaurant une fenêtre thérapeutique. Une interruption de 2 ou 4 demi-vies est recommandée dans la plupart des cas. Elle sera plus longue en cas de chirurgie à haut risque hémorragique et en cas d'insuffisance rénale. Aussi pour les chirurgies mineures, la prise d'AOD est suspendue 24 heures avant l'intervention et reprise 24 heures après (fenêtre thérapeutique de 48 heures). Pour les actes à risque hémorragique élevé, le traitement par AOD est interrompu 5 jours avant l'acte et repris en postopératoire dès que possible une fois le risque hémorragique maîtrisé. Si le risque thrombotique est élevé, un relais par héparine est justifié. Le traitement par une héparine sera alors initié 12 heures après la dernière prise de l'AOD, si celui ci est administré en 2 prises par jour ou 24 heures après si celui ci est administré en une seule prise par jour. En postopératoire, l'AOD sera repris 12 heures après la dernière administration d'HBPM. Dans tous les autres cas, un relais en postopératoire par héparine est en général non nécessaire. Les $A 0 D$ ayant un délai d'action très rapide, il ne doit y avoir aucun chevauchement avec le traitement par l'héparine [259, 260]

Devant l'insuffisance des données de la littérature, le groupe de travail a élaboré les recommandations concernant la gestion des AOD en chirurgie orale à partir d'une position dégradée de celle des AVK.

À la vue des profils d'efficacité et de sécurité des AOD dans les essais de phase III par rapport aux anticoagulants 
de références (warfarine avec un INR cible entre 2 et 3 , enoxaparine $40 \mathrm{mg} 1$ à 2 fois/jour), il était licite de proposer une position similaire à celle adoptée avec les AVK. En cas d'arrêt, la connaissance des paramètres pharmacocinétiques participe à la compréhension de la conduite à tenir (voir annexe 11). Un schéma récapitutatif du protocole d'arrêt et de reprise de L'AOD en cas de chirurgie orale à risque hémorragique élevé est présentée en annexe 12.

- En cas de chirurgie à risque hémorragique faible (en l'absence d'élément jusquà ce jour en faveur d'un arrêt préopératoire même ponctuel), la poursuite des AOD est recommandée. L'intervention est à programmer loin de la dernière prise médicamenteuse, idéalement juste avant la prise suivante correspondant à la concentration résiduelle ou minimale (Cmin).

- En cas de chirurgie à risque hémorragique élevé, l'interruption de l'AOD est indiquée. La durée de cette interruption devrait être évaluée en tenant compte de l'heure et de la posologie de la dernière prise, ainsi que de la fonction rénale. La demi-vie d'élimination des AOD est de 12 heures environ en l'absence d'insuffisance rénale et de 18 heures environ en cas d'insuffisance rénale modérée (clairance de la créatinine comprise entre 30 à $50 \mathrm{~mL} / \mathrm{min}$ ). Une interruption de 2 ou 4 demi-vies est recommandée. En pratique, L'AOD doit être arrêté la veille de l'intervention et repris dans les 24 heures qui suivent l'intervention (fenêtre thérapeutique de 24 à 48 heures). Dans les rares cas de chirurgie à risque élevé chez un patient présentant un risque thrombotique élevé, l'interruption pourra être plus longue (fenêtre thérapeutique de 2 à 5 jours) et un relais par HBPM peut s'avérer nécessaire.

En l'absence d'étude comparative entre AOD et jusqu'à de plus amples informations, il n'y a pas de distinction à faire entre les différentes AOD (dabigatran, rivaroxaban, apixaban) concernant leur gestion périopératoire en chirurgie orale.

Une bonne prise en charge des patients sous AOD en vue d'une intervention chirurgicale ou d'un acte invasif repose principalement sur des critères pharmacologiques tels le nombre de prise par jour, la demi-vie du produit, l'heure de la dernière prise, le délai et la dose de la première dose administrée après la chirurgie [16, 259, 260].

\subsection{Quel test biologique peut-on utiliser pour évaluer le risque hémorragique chirurgical ?}

Si une surveillance de la coagulation n'est pas préconisée en routine, il existe des situations cliniques où l'évaluation peut être utile : chirurgie urgente, accidents thrombotiques, récidives thrombotiques.

Les AOD ont des effets sur les tests de coagulation non spécifiques de routine (temps de Quick ou TQ, temps de céphaline avec activateur ou TCA). La sensibilité de ces tests varie en fonction du médicament et du réactif utilisé. Ces tests usuels sont relativement peu sensibles aux concentrations basses et ont, en fonction du réactif utilisé, une variabilité importante aux concentrations élevées [32, 87, 123, 243, 279]. Aussi la prescription de ces tests globaux de la coagulation n'est pas pertinente pour fournir une estimation du risque hémorragique en vue d'une intervention chirurgicale et/ou d'un geste invasif. Enfin, la mesure de l'INR, mode d'expression du TQ conçu pour les patients traités par AVK, n'a aucun sens chez les patients sous $A O D$ et donc totalement inutile.

En dehors du TQ et du TCA, il existe des tests spécifiques : - le temps de thrombine modifié (Hémoclot ${ }^{\circledR}$, Biophen DTI ${ }^{\circledR}$ ) et le temps d'écarine (ECAT-T ${ }^{\circledR}$ ) pour le dabigatran ;

- la mesure de l'activité anti-Xa (test Rotachrom ${ }^{\circledR}$ anti-FXa, Hyphen Biomed ${ }^{\circledR}$, STA Liquid anti-Xa ${ }^{\circledR}$ ) pour le rivaroxaban et l'apixaban.

L'ensemble de ces tests bien que plus sensibles et/ou précis sont réservés à l'heure actuelle à des centres spécialisés et/ou service d'acceuil des urgences en attendant la connaissance et la validation d'un seuil de sécurité hémostatique autorisant une intervention chirurgicale sans majoration du risque hémorragique pour chacun de ces tests. Ces test ne peuvent être demandés en pratique courante et ne sont pas inscrits à la NABM.

En l'état actuel des connaissances, il n'existe aucun test biologique disponible en routine pour « dépister » les patients sous $A O D$ à risque hémorragique.

\subsection{Quelle hémostase locale doit-on réaliser pour contrô- ler le risque hémorragique chirurgical ?}

En l'absence de données répondant précisément à la question, le groupe de travail recommande l'emploi systématique d'hémostatiques locaux (éponges de gélatine, collagène, fibrine, ou mèche d'oxycellulose) en complément des mesures usuelles (sutures et compression) en cas de chirurgie dentoalvéolaire et du maintien des AOD.

En cas de chirurgie à risque hémorragique élevé et en l'absence de possibilité de d'éffectuer une compression mécanique ou d'une localisation critique, il est impératif de réaliser le geste en situation de sécurité hémostatique en appliquant un protocole d'arrêt de l'AOD.

\subsection{Quelles sont les prescriptions médicamenteuses potentialisatrices du risque hémorragique ?}

Chez les patients traités par A0D, la prescription d'aspirine à fortes doses ( $1 \mathrm{~g}$ par prise ou $3 \mathrm{~g} / \mathrm{jour}$ ), d'AINS (tous), de clarithromycine ou d'antifongiques azolés (tous) par voie systémique n'est pas recommandée car susceptible de majorer le risque hémorragique $[9,10,282]$.

\subsection{Traitement curatif des complications hémorragiques}

Leurs prises en charge reposent toujours en premier lieu sur une reprise chirurgicale et sur la recherche $d^{\prime}$ une cause locale 
de l'hémorragie. L'absence de contrôle durable de l'hémorragie par les mesures usuelles d'hémostase locale est considérée comme un critère de gravité et une prise en charge hospitalière est alors nécessaire. La prise en charge hospitalière des hémorragies graves non contrôlables par une hémostase locale, le GIHP a établi en mars 2013 des propositions dans ce domaine [243]. À l'admission du patient, une mesure de l'exploration de la coagulation est indispensable comprenant un dosage spécifique du dabigatran ou du rivaroxaban et/ou une mesure du TQ et du TCA.

En cas de surdosage, une antagonisation sera discutée. En l'absence d'antidote spécifique, les réversions possibles sont les concentrés de facteurs du complexe prothrombique non activés (ex : CPP) et activés (ex : FEIBA ${ }^{\circledR}$ ) [131], mais les données d'efficacité (arrêt de l'hémorragie) et de sécurité (risque thrombotique) sont très faibles et fonction de la molécule (dabigatran, rivaroxaban) [90]. L'idarucizumab, un anticorps humanisé dirigé contre le dabigatran est actuellement en phase III d'étude clinique [295].

\section{Spécificités de la prise en charge des patients traités par anticoagulants injectables}

\subsection{Les enjeux}

La prévention de la MTEV en chirurgie, en médecine et oncologie ainsi que le traitement curatif de la MTEV à la phase aiguë repose sur la prescription d'anticoagulants administrés par voie parentérale $[4,111]$.

- L'incidence et la durée de la thrombophylaxie varie selon le type de chirurgie. Elle est élevée après PTH, chirurgie de la fracture du col et en chirurgie carcinologique abdominopelvienne. Une thromboprophylaxie prolongée de 4 à 6 semaines est recommandée. Elle est moindre après PTG ainsi que pour les autres types de chirurgie digestive, urologique et gynécologique. Des durées de 7 à 14 jours seraient suffisantes pour ces indications [264]. À noter que la chirurgie orale et maxillo-faciale (non carcinologique) expose à un risque thrombotique faible et ne justifie pas d'une thromboprophylaxie [202].

- La prévention de la MTEV en médecine dépend de l'affection médicale aiguë responsable de l'hospitalisation (insuffisance cardiaque, IDM, cancer évolutif, insuffisance respiratoire décompensée, AVC, maladies inflammatoires....), des facteurs de risque liés au patient (antécédent de MTEV, âge $>60$ ans, IMC > 30, varices, grossesse, contraceptifs oraux) et de la durée d'alitement [4].

- En oncologie, l'incidence de la MTEV varie de 0,5 à $20 \%$ selon le type de cancer. Les thromboses sont fréquentes au cours des cancers du pancréas, des lymphomes, des cancers du tube digestif, de l'ovaire et du poumon. Les AVK sont moins efficaces et moins bien tolérés quand la MTEV survient en présence d'un cancer. Les HBPM sont recommandées pendant au moins 6 mois. Après 6 mois, la poursuite du traitement par une HBPM ou un AVK est décidée en fonction de la tolérance du médicament et de l'évolution du cancer [294].

- L'incidence annuelle de la MTEV en France est de plus de 100000 cas et à l'origine de 5 à 10000 décès. Cette pathologie est une urgence diagnostique et thérapeutique, aussi le traitement curatif doit reposer sur une anticoagulation rapide et efficace. Pour cette raison le traitement à la phase aiguë est une héparine, relayée à distance par un traitement AVK $[4,9,10,80]$.

\subsection{Les anticoagulants injectables}

Les anticoagulants injectables regroupent : les héparines standards ou non fractionnées (HNF), les HBPM, et les autres anticoagulants injectables [111, 124]. (voir annexe 1)

Dans ces recommandations, seules les HNF et les HBPM seront abordées. Sont exclues les autres anticoagulants injectables qui regroupent un ensemble de molécules très hétérogènes : l'inhibiteur sélectif du Xa (fondaripanux ARIXTRA ${ }^{\circledR}$ ), les hirudines recombinantes (Désirudine $\operatorname{REVAC}^{\circledR}$, Bivalirudine ANGIOX ${ }^{\circledR}$, Lépirudine REFLUDAN ${ }^{\circledR}$ ) et le danaparoïde sodique $\left(\mathrm{ORGARAN}^{\circledR}\right)$, avec des indications limitées et réservées à des niches thérapeutiques. Il n'existe aucune donnée dans la littérature sur la gestion péri-opératoire de ces dernières molécules en chirurgie orale.

\subsubsection{Les héparines standards non fractionnées}

L’héparine a été découverte en 1916. Elle potentialise l'antithrombine (AT), inhibiteur physiologique des facteurs Xa et IIa. L'anticoagulation est immédiate. Les HNF combinent une activité anti-IIa et anti-Xa. Il existe deux HNF : l'héparine sodique (HEPARINE CHOAY ${ }^{\circledR}$, HEPARINE PANPHARMA ${ }^{\circledR}$ ) [138] injectable par voie IV en bolus ou en administration continue à la seringue électrique, et l'héparine calcique (CALCIPARINE ${ }^{\circledR}$ ) [136] injectable par voie SC à raison de 2 injections espacées toutes les 12 heures ou en 3 injections espacées de 8 heures $[9,10]$. Après injection IV, la demi-vie d'élimination de l'héparine est de 90 minutes. L'héparinémie disparaît 4 heures après injection IV et 12 heures après injection SC. Son élimination rénale est nulle. Les HNF peuvent ainsi être administrées sans danger chez l'insuffisant rénal et le sujet âgé. Il existe deux grandes indications pour les HNF : le traitement curatif de la MTEV à la phase aiguë, de l'oblitération artérielle aiguë par embolie et de l'IDM à la phase aiguë et le traitement préventif de la MTEV et des thromboses artérielles (anticoagulation des circuits de circulation extra-corporelle, circuits d'hémodialyse) $[9,10]$.

En phase aiguë, l'héparine ne dissout pas le thrombus mais empêche son extension et la migration $d^{\prime}$ embole artériel et veineux. La lyse du thrombus est le fait de la fibrinolyse. 
La prise de HNF requière le plus souvent une hospitalisation et un protocole strict. L'effet indésirable majeur, à côté du risque hémorragique, est le risque de thrombopénie induite par l'héparine (TIH). La TIH est une complication thrombotique, d'origine immuno-allergique, rare mais grave, mettant en jeu le pronostic vital et fonctionnel, nécessitant l'arrêt immédiat de l'héparine. Son risque sous HNF est estimé entre 1 et $5 \%[9,10]$.

\subsubsection{Les héparines fractionnées de bas poids moléclaires}

Les HBPM sont obtenues par dépolymérisation chimique ou digestion enzymatique des chaînes d'HNF La diminution du poids moléculaire des chaines d'héparines ( 3 à 30000 Da pour les HNF et moins de 8000 Da pour les HBPM) confère aux HBPM, comparativement aux HNF, une activité anti-Xa prédominante sur l'activité anti-IIa (rapport variant de 2 à 4 selon les molécules) et un allongement de la demi-vie d'élimination permettant de réduire le nombre d'injection quotidienne à 1 ou 2 injections par jour [111]. Les HBPM sont commercialisées en France depuis 1985 et sont au nombre de quatre $[9,10,282]$ : la daltéparine sodique (FRAGMINE $\left.{ }^{\circledR}\right)[134,153]$, l'énaxoparine sodique (LOVENOX ${ }^{\circledR}$ ) [137], la tinzaparine sodique (INNOHEP ${ }^{\circledR}$ ) [140] et la nadroparine calcique (FRAXIPARINE ${ }^{\circledR}$, FRAXODI $^{\circledR}$ ) [134]. En dehors de l'indication très spécifique du traitement de certaines coagulopathies, les HBPM ont les mêmes indications que les HNF et ont tendance à les remplacer (meilleure tolérance, réduction du nombre d'injections...) [9, 10]. Les HPBM, sont en revanche contre-indiquées en cas d'insuffisance rénale sévère (clairance de la créatinine inférieure à $30 \mathrm{~mL}$ / $\min$ ), alors que les HNF peuvent être utilisées. Le risque de TIH durant un traitement sous HBPM est moindre que sous HNF. Il est estimé à moins de $1 \%$ [6].

Deux contextes d'utilisation des HPBM sont classiquement distinguées : le traitement préventif de la MTEV (chirurgie viscérale, chirurgie orthopédique de la hanche et du genou, alitement pour affection médicale aiguë) et le traitement curatif des TVP constituées, embolie pulmonaire et angor instable/ IDM sans onde $Q$. La durée totale des injections varie selon les indications entre 8 et 35 jours. Dans les situations où l'utilisation des HBPM est déconseillée ou contre-indiquée (ex : insuffisance rénale grave), l'HNF peut être utilisée [282].

\subsection{Arrêt ou maintien du traitement par héparine ?}

La littérature du sujet est pauvre, les études sont peu nombreuses et leurs qualités méthodologiques contestables (voir annexe 13). Ces éléments concourent à ce que le niveau de preuve soit bas, laissant une large part aux avis experts du groupe de travail.

Compte tenu de l'anticoagulation immédiate et de courte durée, (HNF : 4 à 6 heures ; HBPM : 12 heures), deux stratégies peuvent être discutées :

- pas d'arrêt des héparines ;
- arrêt des héparines avant la procédure (6 à 8 heures avant pour les HNF et la veille pour les HBPM et reprise en fonction du contrôle hémostatique).

Une étude rétrospective [168], incluant 41 patients, a évalué le risque de saignement postextractionnel chez des patients sous énoxaparine sodique chez lesquels l'injection précedant la procédure dentaire a été suspendue. Une hémostase locale associant un tamponnement intra-alvéolaire (oxycellulose, gélatine) aux mesures conventionnelles d'hémostase locale (sutures + compression) a été réalisée systématiquement. Aucune complication hémorragique n'est rapportée quelle que soit la dose d'énoxaparine sodique (les doses allaient de $20 \mathrm{mg} /$ jour jusquà $110 \mathrm{mg} /$ jour $\times 2$ fois/jour). En revanche, en cas de bithérapie antithrombotique combinant énoxaparine sodique et warfarine, 3 patients sur 4 ont présenté des complications hémorragiques dont une sévère ayant nécessité une transfusion de plasma frais congelé pour stopper le saignement. Pour ces 3 cas, la valeur de l'INR pratiqué en urgence allait de 1,6 à 2,4.

Une étude comparative randomisée ouverte [33], comparant relais héparinique versus maintien du traitement AVK, rapporte dans le bras relais (arrêt AVK 3 à 4 jours avant la procédure, relais par nadroparine à la dose de 3850 à 5700 UI antiXa en 1 ou 2 injections par jour, suspension de l'injection précédant les extractions dentaires, INR pré-opératoire moyen à $1,26 \pm 0,11$, reprise des AVK et de l'HBPM le soir de l'intervention) un taux de saignement postopératoire de $4,76 \%$. Les saignements étaient de faible intensité et facilement contrôlables par une hémostase locale. Aucune complication hémorragique sévère, ni aucun accident thrombo-embolique dans les 30 jours postopératoires, n'a été signalé. L'analyse de la littérature fait également état de trois cas cliniques de procédure de relais avec une HBPM réalisée en ambulatoire [43, 176, 276]. Parmi ces 3 cas, une complication hémorragique sévère avec saignements répétitifs durant les 24 premières postopératoires et nécessitant une neutralisation par injection de vitamine $\mathrm{K}$ a été décrite [43]. Ce cas clinique souligne l'importance du respect strict du protocole et de l'importance d'une surveillance biologique (INR) durant toute la durée du relais [213].

En cas de relais AVK-héparine-AVK, le choix va préférentiellement aux HBPM compte tenu de leur meilleure tolérance, maniabillité et coût global de la procédure. L'emploi des HNF requiert le plus souvent une hospitalisation, un strict respect du protocole et une surveillance biologique quotidienne. Des doutes subsistent quand à l'efficacité de la protection antithrombotique des HBPM chez les patients à haut risque thrombotique. Des études signalent la survenue de complications thrombotiques durant la période péri-opératoire chez des patients porteurs de valve mécanique [195]. Un ensemble d'études [85, 86, 184, 272-274] montrent néamoins que l'efficacité clinique d'un relais par HBPM à doses curatives en deux injections quotidiennes (hors AMM en France) est comparable à celle obtenue par HNF intraveineuse à la seringue électrique ou par HNF sous cutanée à doses curatives (2 à 3 injections 
par jour). De même, Karsh et coll., 2011 [180], ont montré, chez des patients porteurs de valve mécanique, qu'il n'existait aucune différence significative après extractions dentaires simples, ni en terme de perte sanguine, ni en terme de complications hémorragiques ou thrombotiques entre l'utilisation d'HBPM et de HNF par voie SC.

Globalement en cas de relais héparinique, l'instauration $d^{\prime}$ une fenêtre thérapeutique expose à un thrombotique de $1 \%$ [274] et le chevauchement temporel de la prise de deux anticoagulants (AVK et héparines) présente un risque de saignement sévère d'environ $3 \%$ [274] et de saignement modéré de l'ordre de 7 à $9 \%$ [33, 110, 167]. L'indication d'un relais héparinique doit tenir compte de ces risques encourus et du rapport bénéfice/risque [85, 224].

Une seule étude [226] a évalué le risque de saignement péri- et postopératoire après extraction(s) dentaire(s) en cas de maintien de l'héparinothérapie sans la pratique d'une fénêtre thérapeutique. Il s'agit d'une étude rétrospective ayant inclus 31 patients hospitalisés et traités par HNF en SC ( 2 à 3 injections par jour) pour des affections médicales aiguës sévéres (infarctus cérébral, SCA, thrombus intracardiaque, CIVD, femme enceinte avec EP et TVP). Un taux de saignement postopératoire (non contrôlé par une hémostase locale) de $28,6 \%$ est rapporté. Les complications hémorragiques sont survenues dans les cinq premiers jours postopératoires (médiane à 4 jours). Elles ont été contrôlées par reprise de l'hémostase et ajustement du TCA en dessous de 57 secondes. Dans la moitié des cas, les auteurs ont eu recours à une électrocoagulation ou la pose de gouttière et dans un cas, de la colle de fibrine a été utilisé. Aucune complication sévère avec recours à une transfusion sanguine n'est rapportée.

Au total, après analyse de la littérature, en cas de chirurgie dentoalvéolaire, il semble licite de proposer la poursuite du traitement par héparines (HBPM, HNF) devant l'absence de complication hémorragique sévère.

Dans les cas de chirurgie orale à risque hémorragique élevé, un contact avec le médecin prescripteur est indispensable, afin d'évaluer le rapport bénéfice-risque d'une suspension temporaire de l'héparine. Dans tous les cas, la fenêtre thérapeutique se doit d'être la plus étroite avec une reprise de l'héparine dès que possible.

Dans le cas particulier du relais héparinique chez des patients traités au long cours par AVK, un respect strict du protocole est recommandé. Si la procédure de relais n'est pas réalisée dans un parcours de soins coordonné en ville, il est recommandé d'hospitaliser le patient, au plus tard la veille de la chirurgie pour adapter l'anticoagulation [122].

\subsection{Quel test biologique peut-on utiliser pour évaluer le risque hémorragique chirurgical chez les patients sous héparines?}

- En cas de poursuite du traitement par héparine (HBPM, HNF) et en cas de relais héparinique, une évaluation biologique pré-opératoire est utile afin de verifier l'absence d'une anticoagulabilité excessive et d'avoir une valeur de référence. Le TCA ou l'activité anti-Xa (héparinémie) peuvent être proposés. Dans le cadre d'une surveillance biologique, les valeurs cibles du TCA (pour les HNF) sont entre 2 et 3 fois la valeur du témoin pour un traitement curatif et entre 1,5 et 2 fois la valeur du témoin pour un traitement préventif. Les valeurs cibles de l'activité anti-Xa (pour les HNF et les HBPM) sont entre 0,5 et 1 pour un traitement curatif et entre 0,10 et 0,45 pour un traitement préventif.

- En cas d'arrêt des héparines avant une chirurgie (6 à 8 heures avant pour les HNF et la veille pour les HBPM et reprise en fonction du contrôle hémostatique) les tests de coagulation sont inutiles. C'est le respect du délai minimum entre la dernière injection de l'héparine et le début de l'intervention qui garantit la sécurité hémostatique.

- Compte tenu du risque de TIH, une surveillance plaquettaire systématique à l'instauration du traitement est nécessaire $[4,111,124]$. Il est recommdandé de pratiquer une numération plaquettaire sanguine en début de traitement puis deux fois par semaine pendant 1 mois puis 1 fois par semaine jusqu'à arrêt du traitement [6].

\subsection{Quelle hémostase locale doit-on réaliser pour contrôler le risque hémorragique chirurgical chez les patients sous héparines ?}

D'emblée, il faut noter qu'aucune étude n'a eu pour objectif principal d'évaluer l'efficacité d'une hémostase locale chez des patients sous héparine, ni de comparer les mesures hémostatiques les unes par rapport aux autres.

En cas de traitement aux long cours avec maintien des héparines (HBPM, HNF), l'utilisation d'éponge de gélatine, de collagène ou de gaze d'oxycellulose en tamponement intraalvéolaire en complément des mesures conventionnelles (sutures + compression) est sytématique [167, 226].

En cas de relais héparinique, Bajkin et coll., 2009 [33] ont montré que lorsque l'INR est $<1,5$, la réalisation de sutures n'a pas d'utilité en cas d'extraction(s) dentaire(s) simple(s). Une compression mécanique pendant 30 minutes permet dans $95,24 \%$ des cas d'assurer le contrôle hémostatique. La prise en charge des saignements postopératoires a reposé sur une reprise de l'hémostase avec l'utilisation d'éponge de collagène en complément des mesures conventionnelles (sutures + compression). De même, Karsh et coll., 2011 [180] ne rapportent aucune complication hémorragique postopératoire après tamponnement alvéolaire intra-alvéolaire à l'aide d'oxycellulose complété de sutures et d'une compression mécanique de 1 heure.

\subsection{Traitement curatif des complications hémorragiques chez les patients sous héparines}

Leurs prises en charge reposent toujours en premier lieu sur une reprise chirurgicale et sur la recherche d'une cause locale 
de l'hémorragie. L'absence de contrôle durable de l'hémorragie par les mesures d'hémostase usuelles est considérée comme un critère de gravité et une prise en charge hospitalière est alors nécessaire.

En cas de surdosage (que ce soit pour les HNF et les HBPM), il existe un antidote : le sulfate de protamine. C'est un antagoniste de l'héparine qui s'administre par voie IV. La neutralisation de l'héparine se produit en moins de 5 minutes en formant un complexe inactif. Cent unités anti-héparine (UAH) de sulfate de protamine permettent de neutraliser l'activité anticoagulante de l'héparine $[9,10]$.

\section{Conclusion}

Suivant le potentiel hémorragique, les recommdandations distinguent la chirurgie dento-alvéolaire, des chirurgies orales à risque hémorragique élevé comme la chirurgie pré-implantaire et la chirurgie des kystes et tumeurs bénignes des maxillaires.

Pour la chirurgie dento-alvéolaire, la prise en charge périopératoire des antiplaquettaires et des AVK est validée et parfaitement codifiée. La poursuite du traitement est possible moyennant un certain nombre de précautions (hémostase chirurgicale, conseils et surveillance postopératoires adaptés).

Pour les AOD et les héparines, les données disponibles en 2015 sont très faibles pour définir la meilleure gestion possible vis-à-vis du double risque hémorragique et thrombotique. Dès lors, dans l'attente d'études spécifiques, des propositions dégradées à partir des recommandations établies pour les AVK ont été établies. Elles seront susceptibles d'évoluer en fonction des résultats des études cliniques futures nécessaires.

Pour les chirurgies à haut risque hémorragique, quel que soit le type d'antithrombotique (antiplaquettaire, AVK, AOD, HBPM et HNF), les données disponibles en 2015 sont insuffisantes ou inexistantes pour préciser la prise en charge optimale. Dès lors, le groupe de travail met en avant de la nécessité de contacter le médecin prescripteur afin de définir la meilleure stratégie vis-à-vis du traitement antithrombotique. Une interruption temporaire du médicament antithrombotique avec ou sans mise en place d'un traitement substitutif et une éventuelle prise en charge hospitalière seront à discuter.

Pour chaque grande famille d'antithrombotique (antiplaquettaire, AVK, AOD et héparines), en fonction du risque hémorragique potentiel selon le type de chirurgie et du risque thrombotique lié à l'affection médicale du patient, un algorithme est proposé (voir annexe 14,15,16,17).

Même s'il demeure de nombreuses questions en suspens, essentiellement avec les AOD, la gestion péri-opératoire des patients sous antiplaquettaires et sous AVK s'est considérablement simplifiée. Malgré la simplification des procédures, une prudence s'impose chez les patients très âgés qui cumulent bien souvent de nombreux facteurs associés à une augmentation du risque de saignement. 


\section{ANNEXES}

Annexe 1. Antithrombotiques (antiplaquettaires et anticoagulants) actuellement commercialisés en France en 2015.

\begin{tabular}{|c|c|c|}
\hline \multicolumn{3}{|c|}{ Agents antiplaquettaires } \\
\hline \multicolumn{2}{|c|}{ Voie orale } & Indications (AMM) \\
\hline \multirow[t]{2}{*}{$\begin{array}{l}\text { Inhibiteurs } \\
\text { de la COX-1 }\end{array}$} & $\begin{array}{l}\text { - Inhibiteur réversible } \\
\left.\text { - flurbiprofène (CEBUTID }{ }^{\circledR}\right)\end{array}$ & $\begin{array}{l}\text { - Traitement préventifsecondaire dasn les suites d'un IDM at après déobstruction } \\
\text { (thrombolyseou angioplastie transluminale) chez les patients pour qui un } \\
\text { traitement par aspirine est temporairement contre-indiqué (ex : chirurgie } \\
\text { programmée). Le service medical rendu dans cette indication est insuffisant. }\end{array}$ \\
\hline & 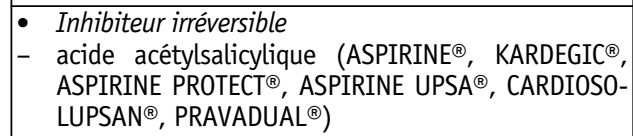 & $\begin{array}{l}\text { - Traitement préventif des accidents thromboemboliques liés à l'athérosclérose } \\
\text { (IDM, AVC) }\end{array}$ \\
\hline \begin{tabular}{l|} 
Inhibiteurs \\
du récepteur $\mathrm{P}_{2} \mathrm{Y}_{12}$ de \\
l'ADP
\end{tabular} & 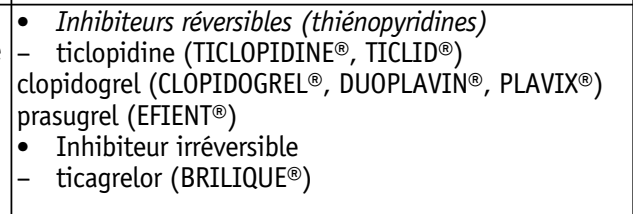 & 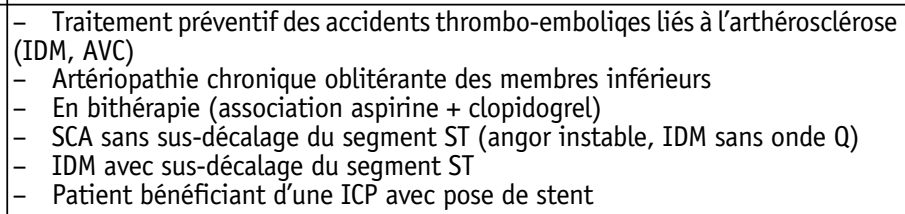 \\
\hline $\begin{array}{l}\text { Inhibiteurs des } \\
\text { phosphodiestérases }\end{array}$ & 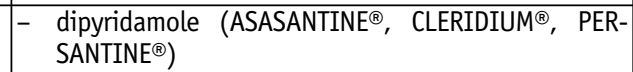 & $\begin{array}{l}\text { - Traitement préventif de l'AVC après un accident ischémique cerebral transitoire } \\
\text { ou constitué, lié à l'athérosclérose, datant de moins de } 3 \text { mois. }\end{array}$ \\
\hline Voie injectable & & Indications (AMM) \\
\hline $\begin{array}{l}\text { Antagonistes du } \\
\text { GPIIb/IIIa }\end{array}$ & 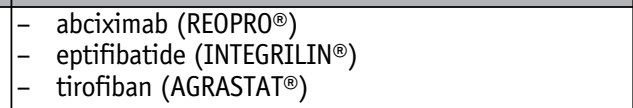 & - ICP à haut risque en périopératoire \\
\hline $\begin{array}{l}\text { Analogue de la } \\
\text { prostacycline (PGI2) }\end{array}$ & - iloprost (ILOMEDINE ${ }^{\circledR}$, VENTAVIS $^{\circledR}$ ) & $\begin{array}{l}\text { Traitement de l'hypertension artérielle pulmonaire idiopathique chez les } \\
\text { patients en classe fonctionnelle III }\end{array}$ \\
\hline \multicolumn{3}{|l|}{ Anticoagulants } \\
\hline \multicolumn{2}{|l|}{ Voie orale } & Indications (AMM) \\
\hline $\begin{array}{l}\text { Antagonistes de la } \\
\text { vitamine K } \\
\text { (inhibition des } \\
\text { facteurs II, VII, IX, X) }\end{array}$ & $\begin{array}{ll}- & \text { acénocoumarol (MINI-SINTROM }{ }^{\circledR}, \text { SINTROM }^{\circledR} \text { ) } \\
\text { - } & \text { fluindione ( PREVISCAN }{ }^{\circledR} \text { ) } \\
\text { - } & \text { warfarine (COUMADINE }{ }^{\circledR} \text { ) }\end{array}$ & $\begin{array}{ll}\text { - } & \text { Traitement préventif et curatif de la MTEV } \\
\text { - } & \text { Cardiopathies emboligènes (prothèses valvulaires, valvulopathies, ACFA) } \\
\text { - } & \text { Infarctus du myocarde compliqué d'insuffisance cardiaque ou trouble du rythme } \\
\text { - } & \text { Embolies systémiques récidivantes }\end{array}$ \\
\hline $\begin{array}{l}\text { Inhibiteurs de la } \\
\text { thrombine (anti-IIa) }\end{array}$ & - dabigatran (PRADAXA $\left.{ }^{\circledR}\right)$ & - Traitement préventif des embolies systémiques dans l'ACFA sans valvulopathie \\
\hline \multirow[t]{2}{*}{$\begin{array}{l}\text { Inhibiteurs du facteur } \\
\text { X activé (anti-Xa) }\end{array}$} & - apixaban (ELIQUIS $\left.{ }^{\circledR}\right)$ & $\begin{array}{l}\text { - Traitement préventif des évènements thromboemboliques veineux après } \\
\text { chirurgie orthopédique } \\
\text { - Traitement préventif des embolies systémiques dans l'ACFA sans valvulopathie }\end{array}$ \\
\hline & - rivaroxaban (XARELT0 ${ }^{\circledR}$ ) & $\begin{array}{ll}- & \text { Traitement préventif de la MTEV en chirurgie orthopédique } \\
\text { - } & \text { Traitement curatif de la TVP et de l'EP } \\
\text { - } & \text { Traitement préventif des embolies systémiques dans l'ACFA sans valvulopathie }\end{array}$ \\
\hline \multicolumn{2}{|l|}{ Voie injectable } & Indications (AMM) \\
\hline \multirow[t]{2}{*}{$\begin{array}{l}\text { Héparines } \\
\text { (Inhibiteurs des } \\
\text { facteurs IIa et Xa) }\end{array}$} & $\begin{array}{l}- \text { Héparines standards ou non fractionnées (HNF) } \\
\text { - héparine sodique (HEPARINE CHOAY, PANPHARMA }{ }^{\circledR} \text { ) } \\
\text { - héparine calcique (CALCIPARINE }{ }^{\circledR} \text { ) }\end{array}$ & $\begin{array}{l}\text { - Traitement préventif des accidents thrombo-emboliques artériels et veineux } \\
\text { - Traitement curatif : MTEV, syndrome coronarien aigu, embolies artérielles } \\
\text { extracérébrales }\end{array}$ \\
\hline & $\begin{array}{l}-\quad \text { Héparines de bas poids moléculaire, voie sous-cutanée } \\
(\text { HBPM) } \\
\left.-\quad \text { daltéparine sodique (FRAGMINE }{ }^{\circledR}\right) \\
-\quad \text { enoxaparine sodique (LOVENOX) } \\
-\quad \text { nadroparine calcique (FRAXIPARINE }{ }^{\circledR}, \text { FRAXODI }^{\circledR} \text { ) } \\
-\quad \text { tinzaparine sodique (INNOHEP }{ }^{\circledR} \text { ) }\end{array}$ & $\begin{array}{l}\text { - Traitement préventif de la MTVE en chirurgie et en médecine } \\
\text { - } \text { Traitement curatif des TVP et/ou EP } \\
\text { - Syndrome coronarien aigu non ST sus } \\
\text { - Hémodialyse rénal en prévention de la coagulation dans le circuit } \\
\text { extracorporel }\end{array}$ \\
\hline \multirow[t]{5}{*}{$\begin{array}{l}\text { Autres } \\
\text { anticoagulants } \\
\text { injectables }\end{array}$} & $\begin{array}{ll} & \text { Pentasaccharide } \\
\text { - } & \text { fondaparinux (ARIXTRA }{ }^{\circledR} \text { ) }\end{array}$ & $\begin{array}{l}\text { - Traitement préventif de la MTVE en chirurgie orthopédique, en chirurgie } \\
\text { abdomidale chez des patients à haut risque (cancer) ou chez les patients jugés } \\
\text { à haut risque alités } \\
\text { - Traitement curatif des TVP et/ou EP } \\
\text { - Syndrome coronarien aigu non ST sus }\end{array}$ \\
\hline & $\begin{array}{ll} & \text { Héparinoïdes } \\
\text { - } & \left.\text { danaparoïde (ORGARAN }{ }^{\circledR}\right)\end{array}$ & $\begin{array}{l}\text { Traitement préventif et curatif des accidents thrombo-emboliques artériels } \\
\text { et veineux chez des patients ayant des ATCD ou atteints de TIH de type II }\end{array}$ \\
\hline & $\begin{array}{l}- \text { Hirudines recombinantes } \\
\left.\text { - } \text { bivalirudine (ANGIOX }{ }^{\circledR}\right)\end{array}$ & - - Anticoagulants chez les patients subissant une ICP en périopératoire \\
\hline & - désirudine (REVASC ${ }^{\circledR}$ ) & - Traitement préventif des TVP après chirurgie orthopédique \\
\hline & - lépirudine (REFLUDAN ${ }^{\circledR}$ ) & $\begin{array}{l}\text { - Traitement curatif des accidents thrombo-emboliques artériels et vei- } \\
\text { neux chez des patients ayant des ATCD ou atteints de TIH de type II }\end{array}$ \\
\hline
\end{tabular}


Annexe 2. Stratification du risque hémorragique en fonction du type de chirurgie et mesures préventives.

\begin{tabular}{|c|c|}
\hline Types de chirurgies et actes invasifs & Mesures préventives des complications hémorragiques \\
\hline \multicolumn{2}{|l|}{ Actes sans risque hémorragique } \\
\hline $\begin{array}{l}\text { - } \\
\text { - } \\
\text { Détartrage }\end{array}$ & - Hémostase mécanique simple par pression \\
\hline \multicolumn{2}{|l|}{ Chirurgies et actes à faible risque hémorragique } \\
\hline \multicolumn{2}{|c|}{ (Chirurgies pour lesquelles une hémorragie extériorisée est facilement contrôlable par une hémostase chirurgicale conventionnelle*) } \\
\hline 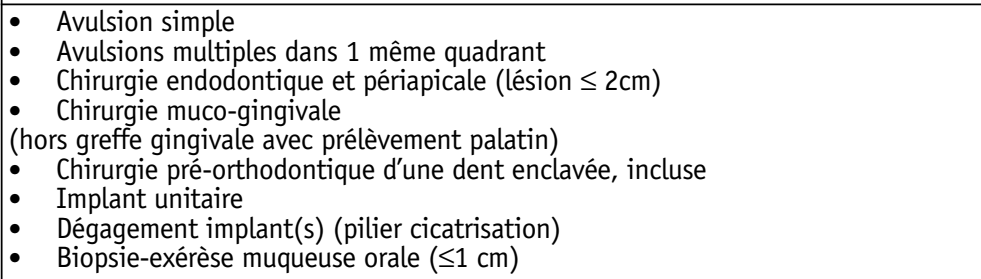 & $\begin{array}{ll}\text { - } & \text { Mesure d'hygiène bucco-dentaire et détartrage } \\
\text { - } & \text { Hémostase chirurgicale conventionnelle } \\
\text { - } & \text { Acide tranexamique }\end{array}$ \\
\hline \multicolumn{2}{|l|}{ Chirurgies et actes invasifs à risque hémorragique élevé } \\
\hline \multicolumn{2}{|c|}{$\begin{array}{l}\text { (Chirurgies pour lesquelles des pertes sanguines significatives et/ou transfusions plaquettaires sont rapportées dans la littérature, interventions d'une durée } \\
\text { opératoire }>1 \text { heure, interventions critiques par leurs localisations (sinus maxillaire, plancher buccal) et/ou difficilement contrôlables par une hémostase } \\
\text { chirurgicale conventionnelle*). }\end{array}$} \\
\hline 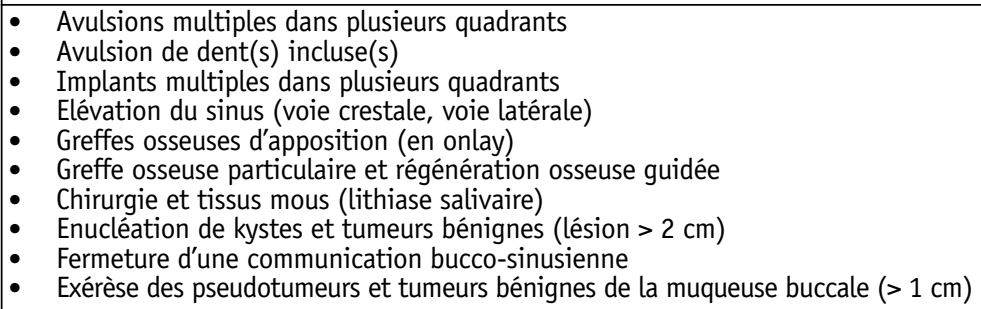 & $\begin{array}{l}\text { - Même mesure préventive que pour une chirurgie à risque hémor- } \\
\text { ragique faible à modéré } \\
\text { - Médicaments dérivés du sang, à base de fibrinogène et thrombine } \\
\text { humains } \\
\text { - Electrocoagulation mono et bipolaire } \\
\text { - Privilégier les chirurgies mini-invasives (flapless et chirurgie } \\
\text { implantaire guidée, abord du sinus par voie crestale...) } \\
\text { - Imagerie 3D préopératoire (sinus, région symphysaire) en cas de } \\
\text { pose d'implant }\end{array}$ \\
\hline \multicolumn{2}{|l|}{ Gestes déconseillés } \\
\hline \multicolumn{2}{|c|}{$\begin{array}{l}\text { - Bloc du nerf alvéolaire inférieur : déconseillé } \\
\text { - Greffe autologue : déconseillée en raison d'un site de prélèvement supplémentaire, privilégier les greffons hétérologues et synthétiques }\end{array}$} \\
\hline \multicolumn{2}{|c|}{ Gestes contre-indiqués } \\
\hline \multicolumn{2}{|c|}{ 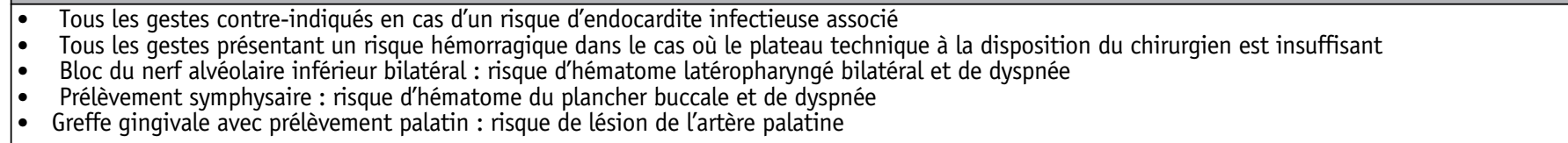 } \\
\hline
\end{tabular}

* hémostase chirurgicale conventionnelle : hémostase mécanique (pression+ sutures) \pm hémostatiques locaux résorbables (éponges collagène ou gélatine, gaze de cellulose) \pm colle synthétique (colle de cyanoacrylate).

Facteurs majorant le risque hémorragique opératoire : décollement muco-périosté au délà de la ligne muco-gingivale, décollement lingual, avulsion(s) en zone inflammatoire, parodonte amoindri, durée opératoire $>1 \mathrm{~h}$ (perte sanguine significative).

Localisations critiques : plancher buccal, symphyse mentonnière, sinus maxillaire.

Annexe 3. Âge et comorbidités associés à une augmentation du risque d'événement hémorragique chez les patients traités par un antithrombotique.

\begin{tabular}{|l|}
\hline Facteurs de risque de saignement spontané ou provoqué \\
\hline Âge : plus fréquent après 65 ans et plus grave après 75 ans \\
\hline Faible masse pondérale (chez l'adulte poids $<50 \mathrm{Kg}$ ) \\
\hline Perte d'autonomie et/ou absence de coopération (patient à mobilité réduite, handicapé, sous tutelle ....) \\
\hline Dysfonction rénale sévère (dialyse, transplantation rénale, insuffisance rénale sévère : clairance de la créatinine $<20 \mathrm{ml} / \mathrm{mn}$ ) \\
\hline Insuffisance cardiaque sévère \\
\hline Hypertension artérielle (HTA) mal contrôlée (pression systolique > $160 \mathrm{mmHg})$ \\
\hline Anémie \\
\hline $\begin{array}{l}\text { Pathologie intercurrente modifiant le métabolisme des antithrombotiques et risquant de provoquer un surdosage : hépatopathie chronique (cirrhose) ou bio- } \\
\text { logique (bilirubine > à } 2 \text { fois la normale associée à ASAT/ALAT > 3 fois la normale) ou destruction de la flore intestinale (antibiotiques, diarrhée) }\end{array}$ \\
\hline Association avec des médicaments à l'origine d'une potentialisation des molécules antithrombotiques (AINS, Millepertuis, antifongiques azolés, vérapamil ....) \\
\hline INR instable \\
\hline Consommation excessive d'alcool \\
\hline
\end{tabular}


Annexe 4. Evaluation du risque de saignement et du risque thrombo-embolique postopératoires en fonction de la stratégie thérapeutique après chirurgie dento-alvéolaire.

\begin{tabular}{|c|c|c|c|c|}
\hline Antithrombotiques(s) & \multicolumn{3}{|c|}{$\begin{array}{l}\text { Risque de saignement } \\
\text { postopératoire }\end{array}$} & $\begin{array}{l}\text { Risque thrombotique postopératoire } \\
\text { (30 jours) }\end{array}$ \\
\hline \multicolumn{5}{|l|}{ Antiplaquettaire } \\
\hline $\begin{array}{l}\text { Arrêt de l'aspirine, } \\
\text { clopidogrel }\end{array}$ & 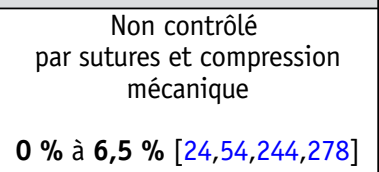 & & & $\begin{array}{c}0,005 \%-5,4 \%[13,64,65,102,278] \\
\text { décès rapportés }[278]\end{array}$ \\
\hline $\begin{array}{l}\text { Poursuite de l'aspirine, } \\
\text { clopidogrel }\end{array}$ & $\begin{array}{c}\text { Non contrôlé } \\
\text { par sutures et compression } \\
\text { mécanique } \\
0 \% \text { à - } 15,7 \%[54,185,187, \\
204,212]\end{array}$ & 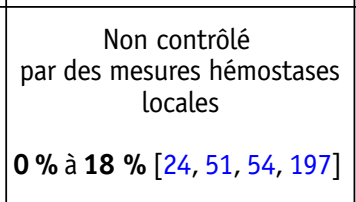 & $\begin{array}{c}\text { Aucune complication } \\
\text { hémorragique sévère } \\
\text { rapportée }\end{array}$ & \\
\hline $\begin{array}{l}\text { Poursuite de l'aspirine + } \\
\text { clopidogrel }\end{array}$ & $\begin{array}{c}\text { Non contrôlé } \\
\text { par sutures et compression } \\
\text { mécanique } \\
1,7 \% \text { à } 66,7 \%[36,54,197, \\
234,239,248]\end{array}$ & $\begin{array}{c}\text { Non contrôlé } \\
\text { par des mesures hémostases } \\
\text { locales } \\
0 \% \\
0 \% \\
{[36,54,197,227,234,239} \\
248]\end{array}$ & $\begin{array}{c}\text { Aucune complication } \\
\text { hémorragique sévère } \\
\text { rapportée }\end{array}$ & \\
\hline \multicolumn{5}{|l|}{ Antivitamines $\mathrm{K}$} \\
\hline $\begin{array}{l}\text { Arrêt de l'AVK ( } 2 \text { à } 5 \text { jours } \\
\text { avant procédure) }\end{array}$ & $\begin{array}{c}\text { Non contrôlé } \\
\text { par sutures et compression } \\
\text { mécanique } \\
14 \%[99]\end{array}$ & & $\begin{array}{c}\text { Hémorragique sévère } \\
0,2 \%[283]\end{array}$ & $\begin{array}{l}0,5 \%[283] \\
0,4 \% \text { si arrêt fenêtre thérapeutique } \\
\leq 5 \text { jours } \\
2,2 \% \text { si arrêt fenêtre thérapeutique } \\
\geq 7 \text { jours } \\
\text { décès rapportés }[31,244,278]\end{array}$ \\
\hline $\begin{array}{l}\text { Arrêt de l'AVK ( } 2 \text { jours } \\
\text { avant procédure) et relais } \\
\text { par HNF }\end{array}$ & $\begin{array}{c}\text { Non contrôlé } \\
\text { par sutures et compression } \\
\text { mécanique } \\
15 \text { à } 36 \%[226,269]\end{array}$ & & & \\
\hline $\begin{array}{l}\text { Arrêt de l'AVK ( } 4 \text { à } 5 \text { jours } \\
\text { avant procédure) et relais } \\
\text { par HBPM }\end{array}$ & $\begin{array}{c}\text { Non contrôlé } \\
\text { par sutures et compression } \\
\text { mécanique } \\
\mathbf{7 , 3} \%[33]\end{array}$ & $\begin{array}{c}\text { Non contrôlé } \\
\text { par des mesures hémostases } \\
\text { locales } \\
\mathbf{4 , 7 6 \% [ 3 3 ]}\end{array}$ & $\begin{array}{c}\text { Hémorragique sévère } \\
3,7 \% \text { [283] }\end{array}$ & \\
\hline $\begin{array}{l}\text { Poursuite de l'AVK et INR } \\
\leq 4\end{array}$ & $\begin{array}{c}\text { Non contrôlé } \\
\text { par sutures et compression } \\
\text { mécanique } \\
13 \% \text { à } 40 \% \\
{[20,46,249,254,263]}\end{array}$ & $\begin{array}{c}\text { Non contrôlé } \\
\text { par des mesures hémostases } \\
\text { locales } \\
0 \% \text { à } 26 \%[28,33,41,46, \\
51,55,82,99,114,115,126, \\
209,249,254,255,263,283]\end{array}$ & $\begin{array}{c}\text { Hémorragie sévère, } \\
\\
0 \% \text { à } 2,77 \% \\
{[28,33,41,46,51,55,} \\
82,99,114,115,126, \\
209,249,254,255, \\
263,283]\end{array}$ & \\
\hline $\begin{array}{l}\text { Poursuite de l'AVK } \\
(\text { INR }<4)+\text { AAP }\end{array}$ & & \begin{tabular}{|c|} 
Non contrôlé \\
par des mesures hémostases \\
locales \\
$\mathbf{3 , 9} \%$ à $\mathbf{8 , 2} \%[35,223,225]$
\end{tabular} & & \\
\hline \multicolumn{5}{|c|}{ Héparines au long cours (patients hospitalisés) } \\
\hline$H N F( \pm A V K \pm A A P)$ & & $\begin{array}{c}\text { Non contrôlé } \\
\text { par des mesures hémostases } \\
\text { locales } \\
\mathbf{2 8 , 6} \%[226]\end{array}$ & & \\
\hline HBPM ( \pm AVK ou AAP) & & $\begin{array}{c}\text { Non contrôlé } \\
\text { par des mesures hémostases } \\
\text { locales } \\
\mathbf{7 , 1 \% [ 1 6 7 ]}\end{array}$ & & \\
\hline
\end{tabular}

Patients ne prenant pas d'antithrombotique : risque de saignement postopératoire : 0 à $2 \%$ [20, 28, 283]. 


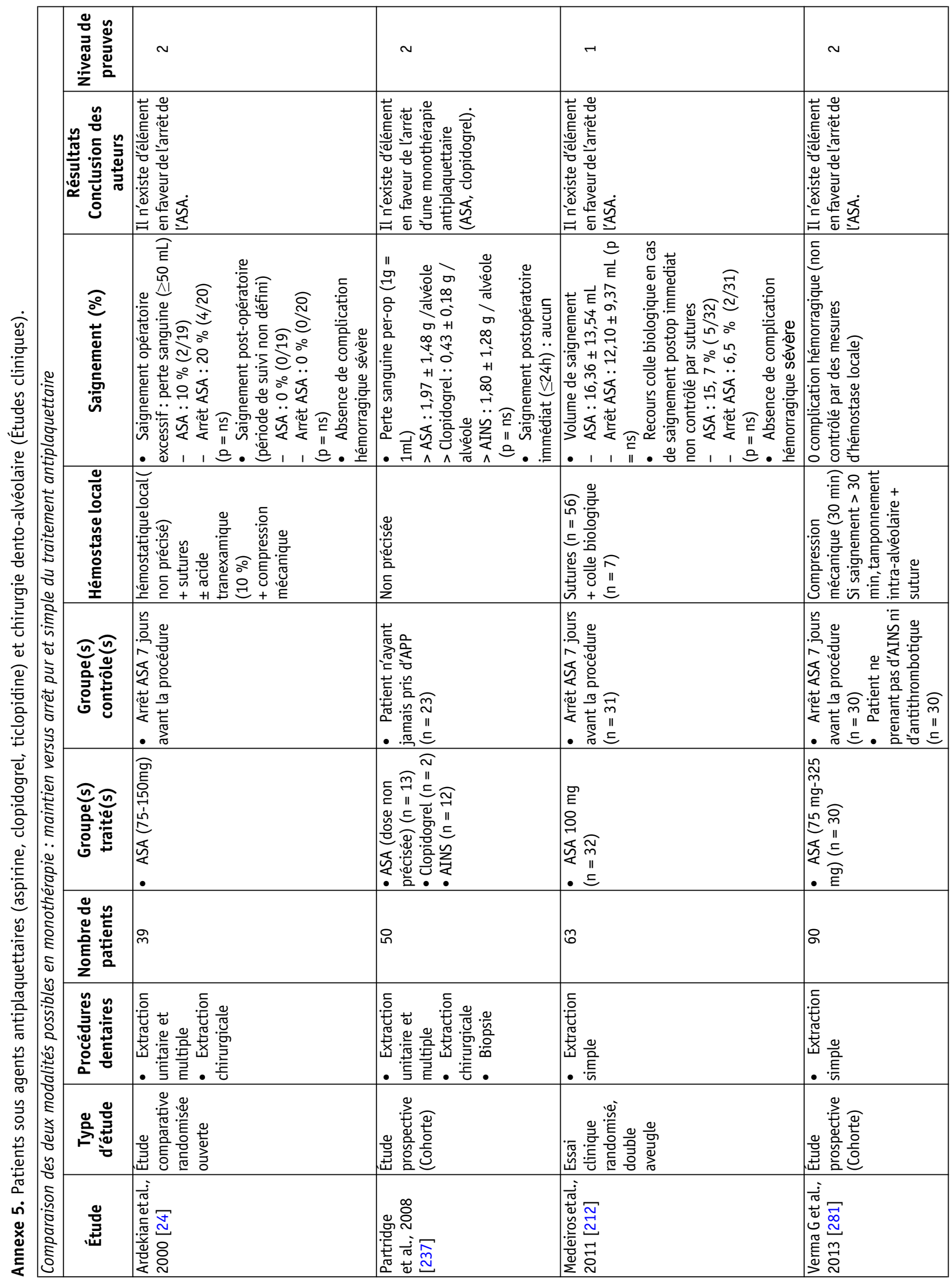




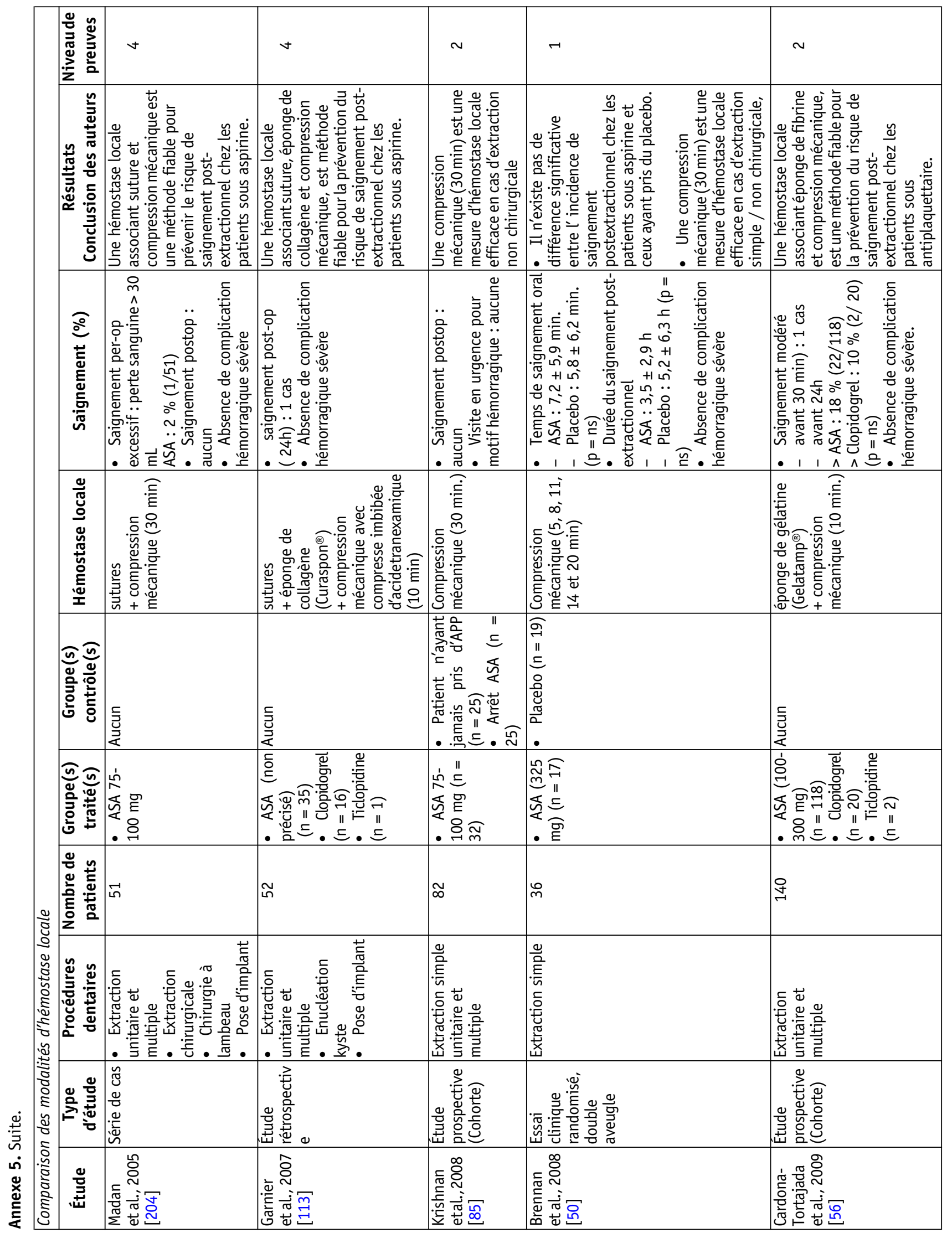




\begin{tabular}{|c|c|c|c|c|}
\hline 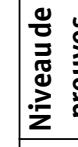 & \multicolumn{2}{|l|}{$\checkmark$} & $\checkmark$ & $\sim$ \\
\hline 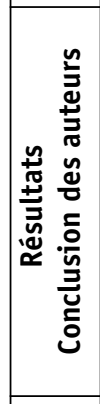 & 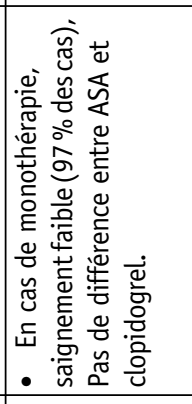 & 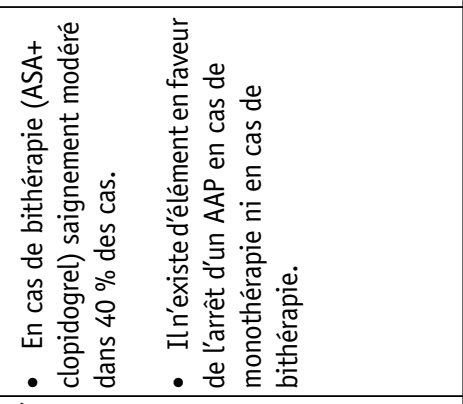 & 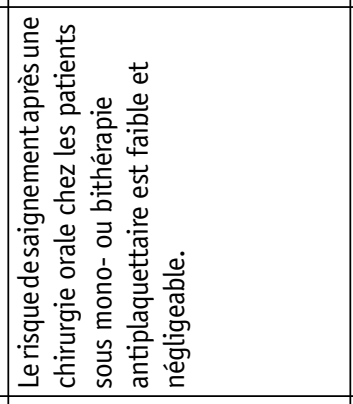 & 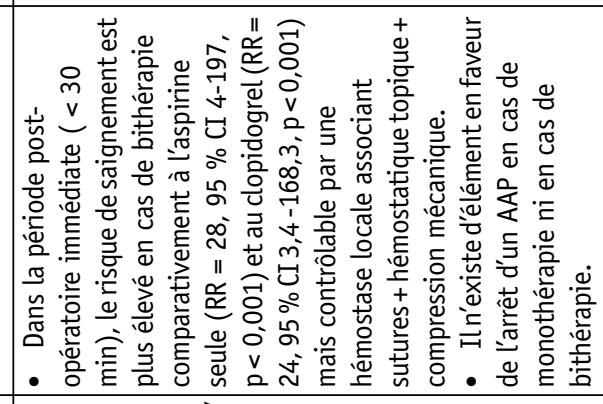 \\
\hline 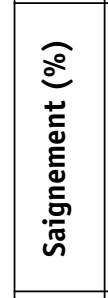 & 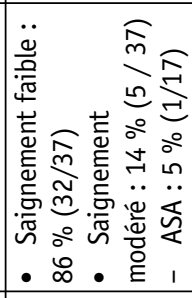 & 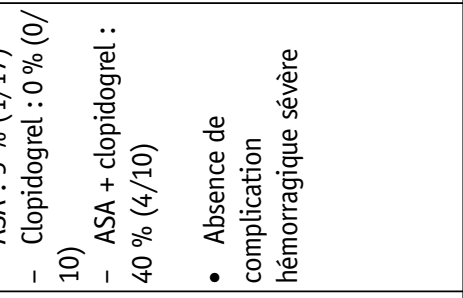 & 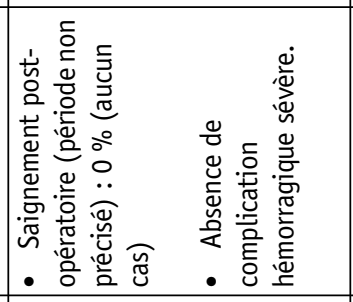 & 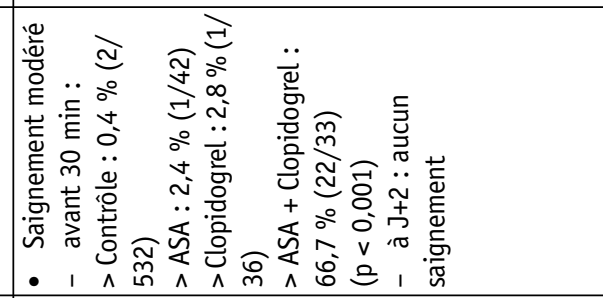 \\
\hline 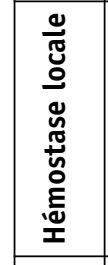 & 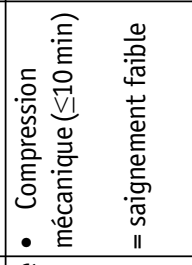 & 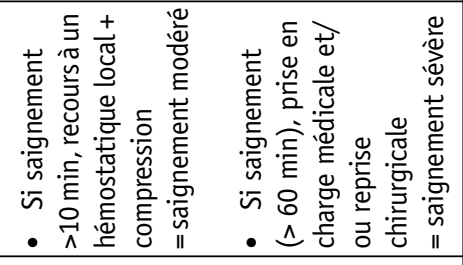 & 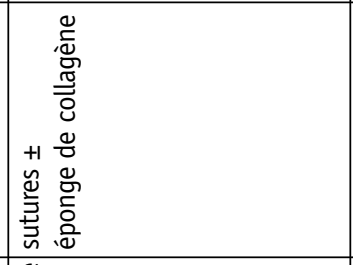 & 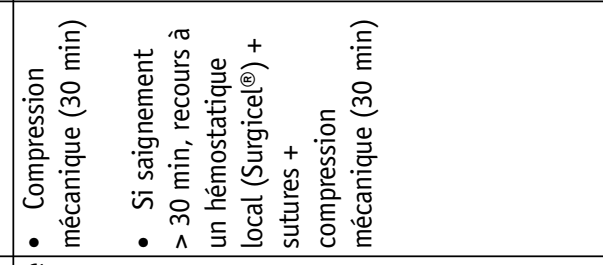 \\
\hline 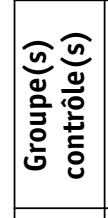 & 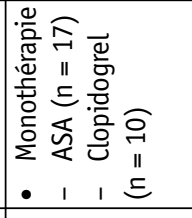 & & 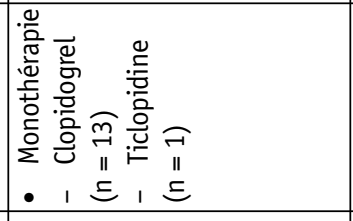 & 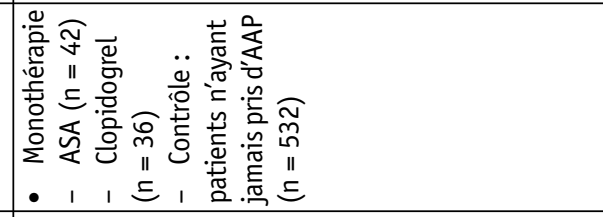 \\
\hline 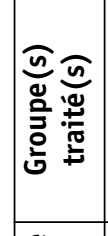 & 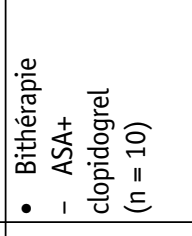 & & 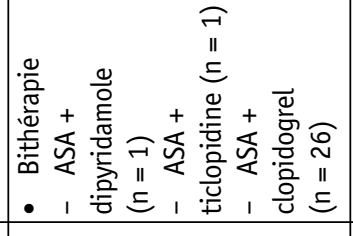 & 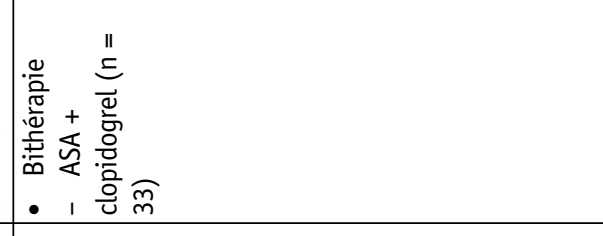 \\
\hline 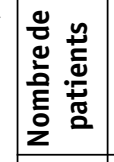 & $\stackrel{\circ}{m}$ & & F & ఫ్ \\
\hline 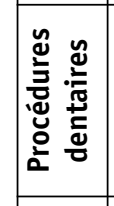 & 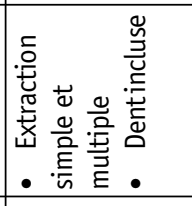 & & 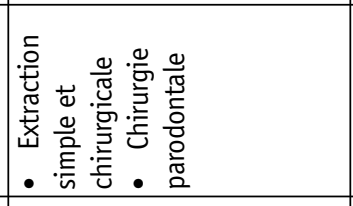 & 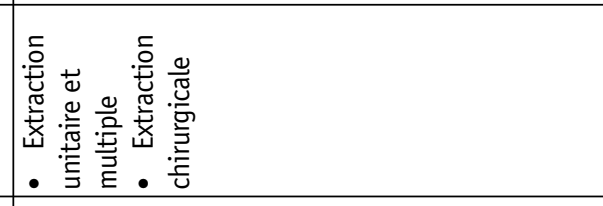 \\
\hline 总 & 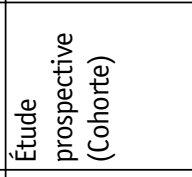 & & 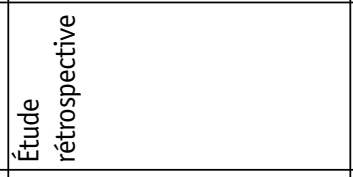 & 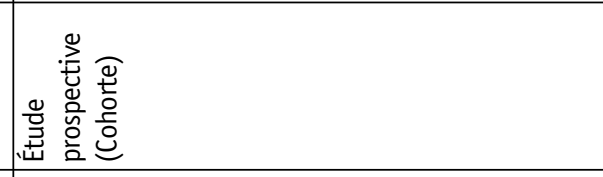 \\
\hline 吾 & 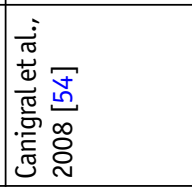 & & 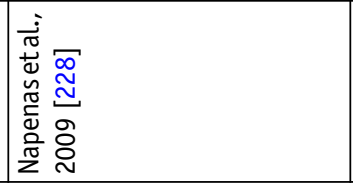 & 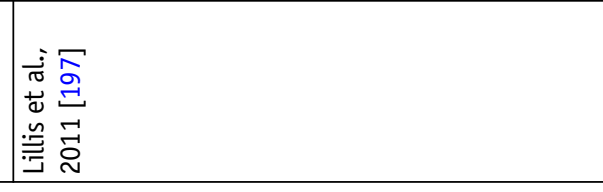 \\
\hline
\end{tabular}




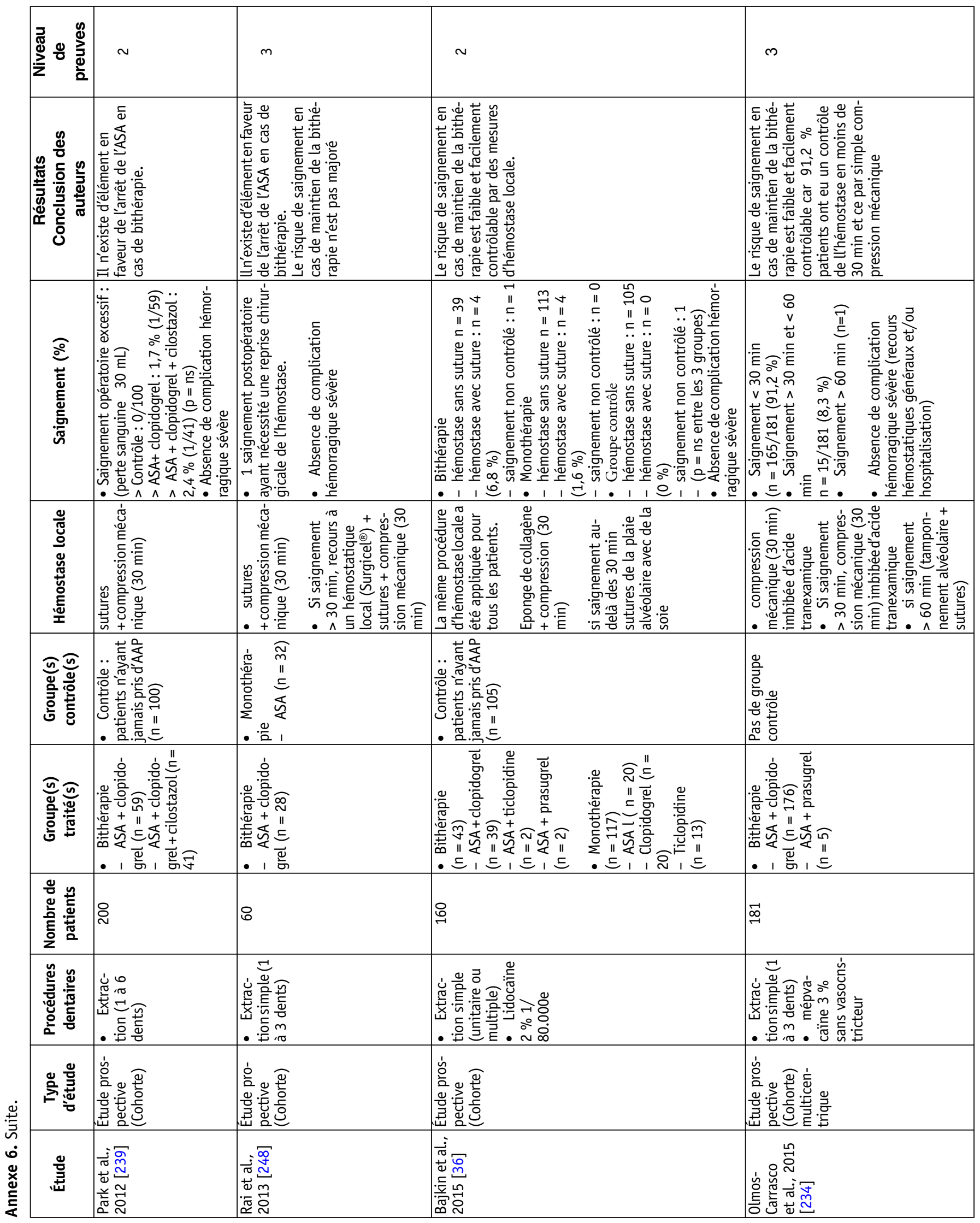




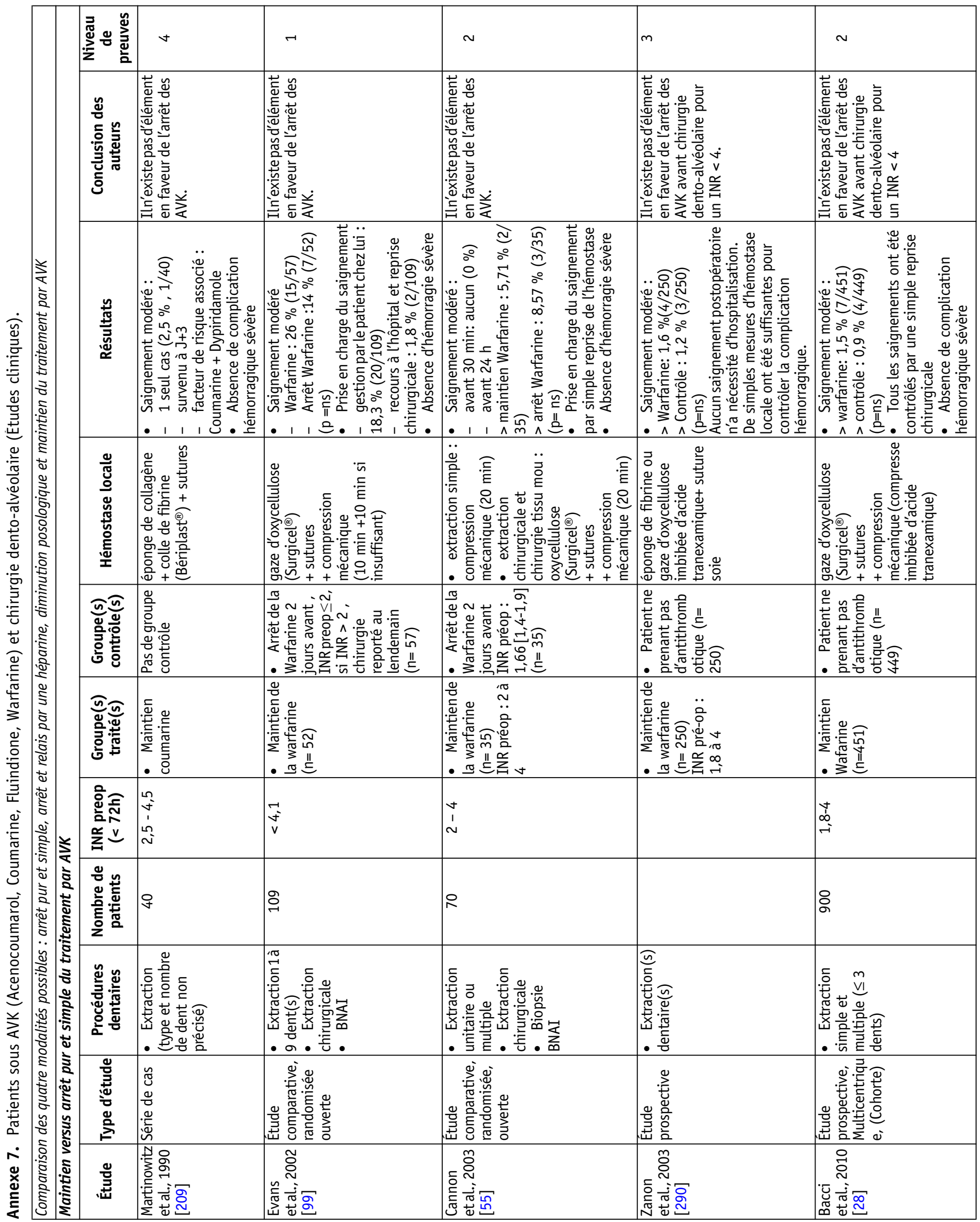




\begin{tabular}{|c|c|c|c|c|c|c|c|}
\hline & 放 & $m$ & & 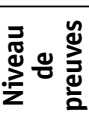 & $\sim$ & $\sim$ & $\sim$ \\
\hline & 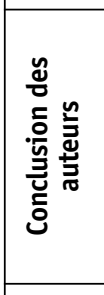 & 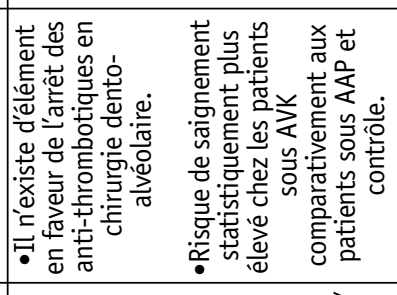 & & 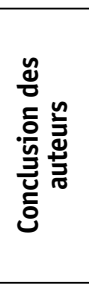 & 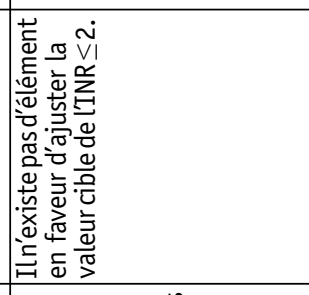 & 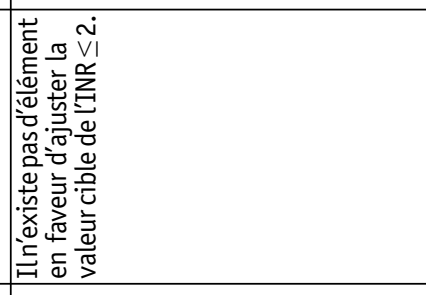 & 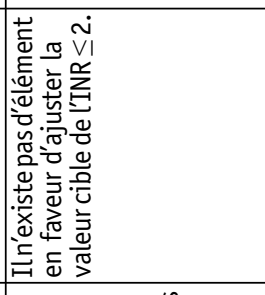 \\
\hline 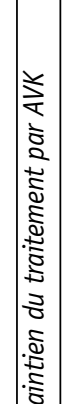 & 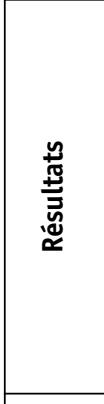 & 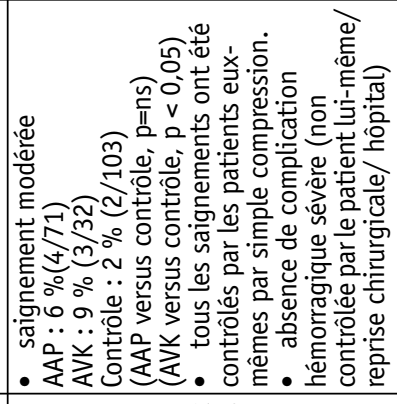 & & 䔍 & 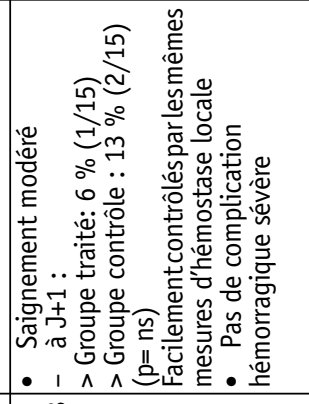 & 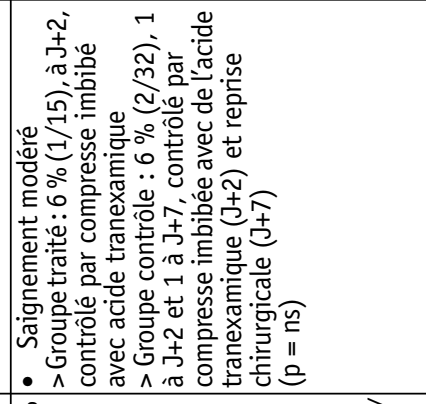 & 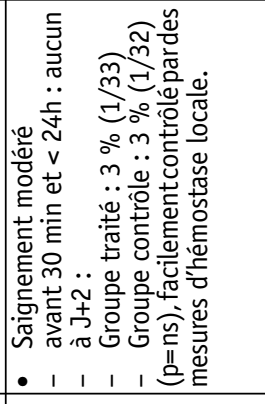 \\
\hline 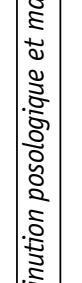 & 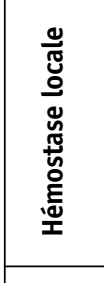 & 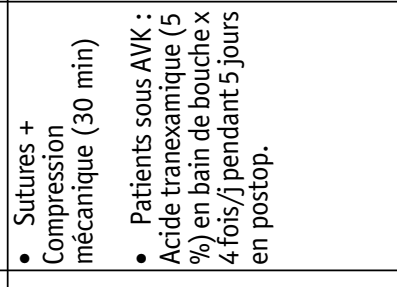 & & 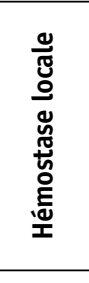 & 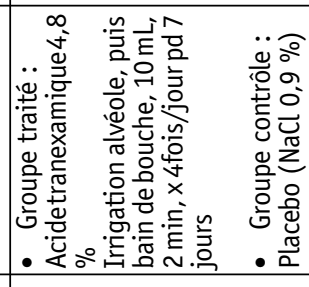 & 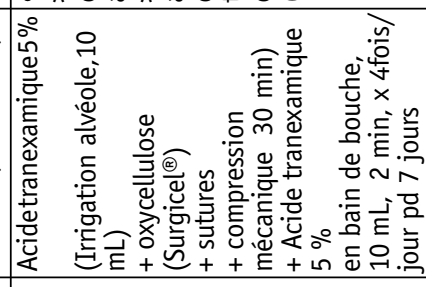 & 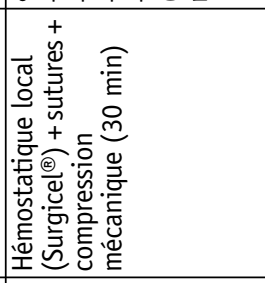 \\
\hline $\mid$ & 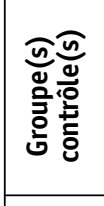 & 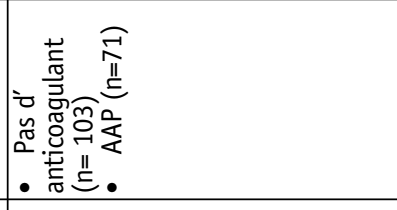 & & 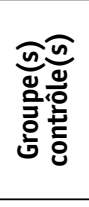 & 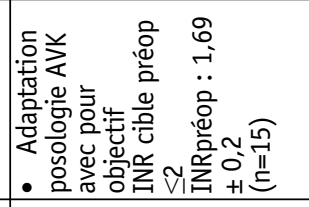 & 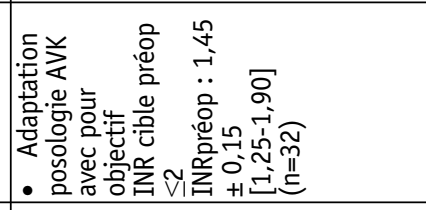 & 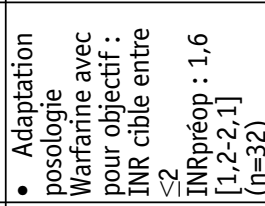 \\
\hline 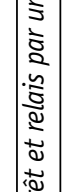 & 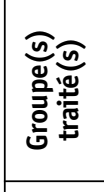 & 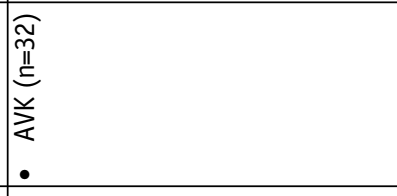 & & 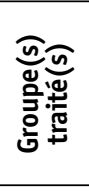 & 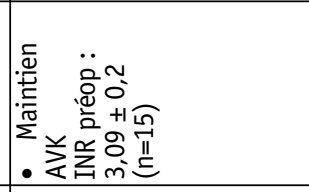 & 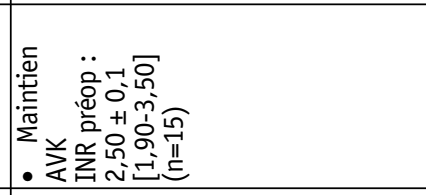 & 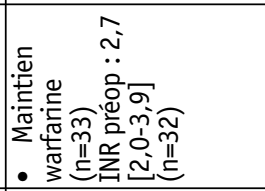 \\
\hline 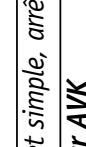 & 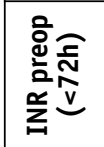 & $\mid \begin{array}{l}n \\
m \\
m \\
\infty \\
-i\end{array}$ & 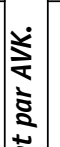 & 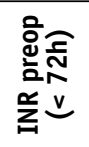 & $\begin{array}{l}\vec{v} \\
\mathrm{v}\end{array}$ & $\stackrel{v}{v}$ & $\underset{\sim}{+}$ \\
\hline 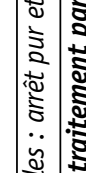 & 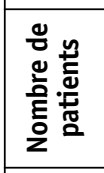 & ¿ః & 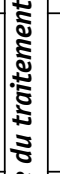 & 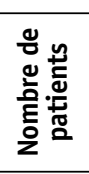 & 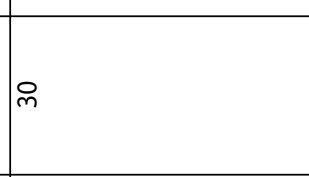 & f & గొ \\
\hline 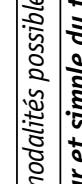 & 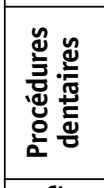 & 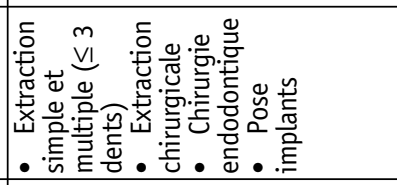 & 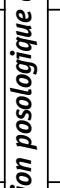 & 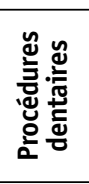 & 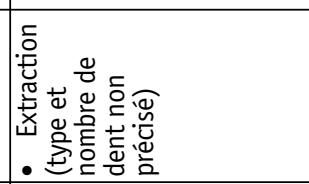 & 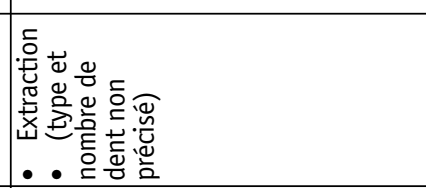 & 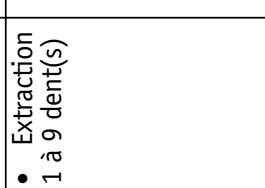 \\
\hline 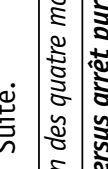 & 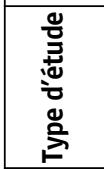 & 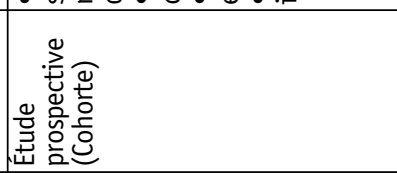 & $\mid$ & 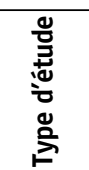 & 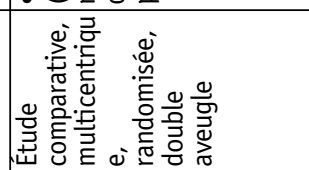 & 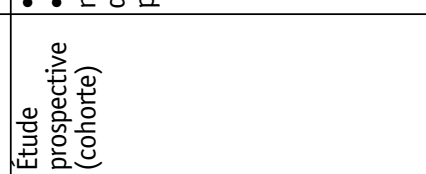 & 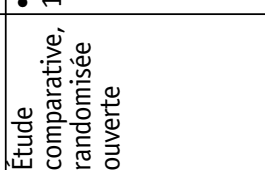 \\
\hline 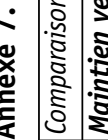 & 莡 & 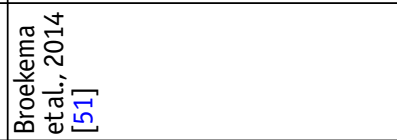 & 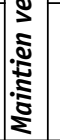 & 莹 & 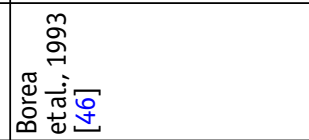 & 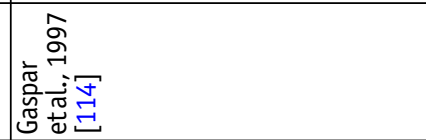 & 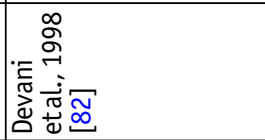 \\
\hline
\end{tabular}




\begin{tabular}{|c|c|c|c|c|c|c|}
\hline & \multirow{2}{*}{ 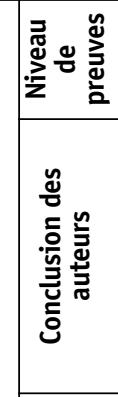 } & \multicolumn{2}{|l|}{4} & \multirow{2}{*}{ 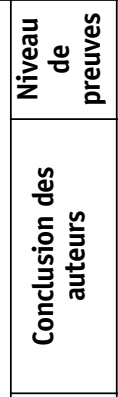 } & \multirow{2}{*}{ 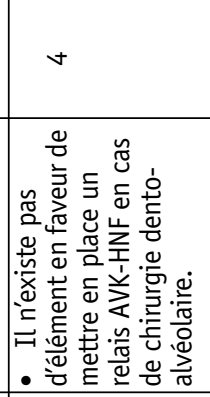 } & \multirow{2}{*}{ 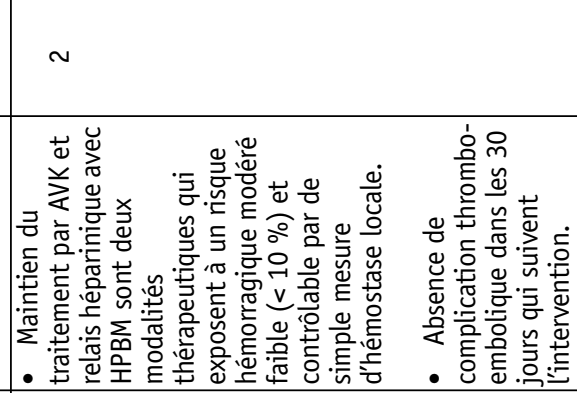 } \\
\hline & & 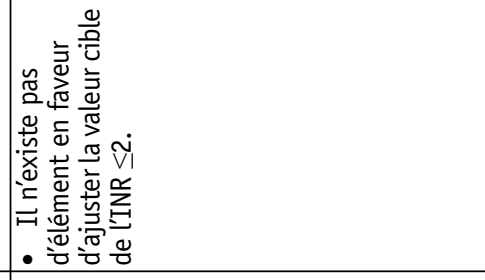 & & & & \\
\hline & 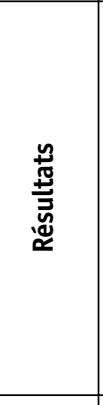 & 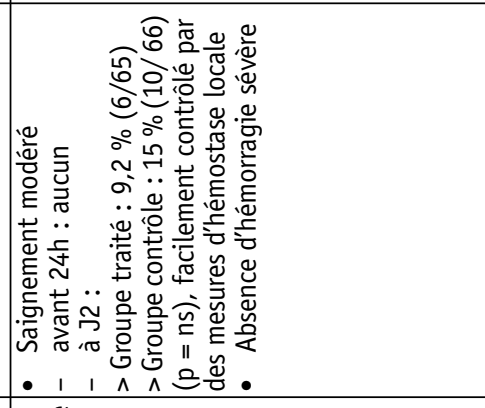 & & 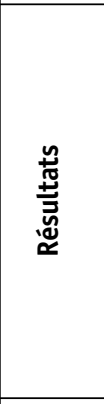 & 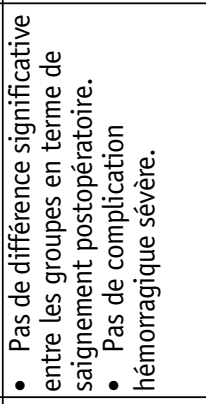 & 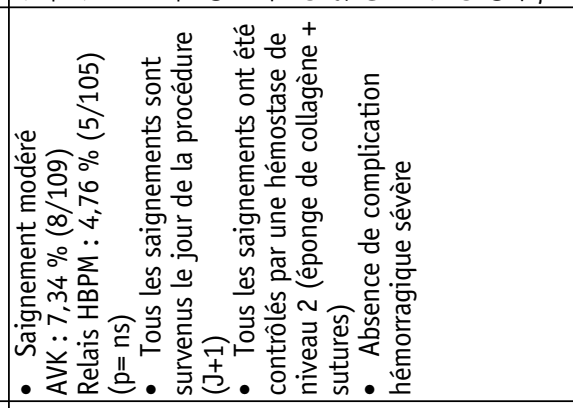 \\
\hline & 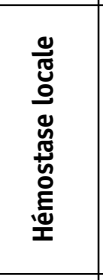 & 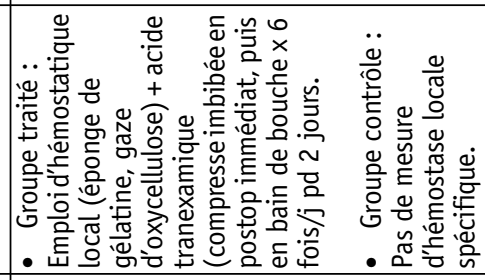 & & 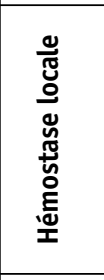 & 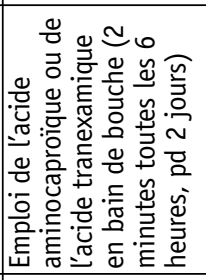 & 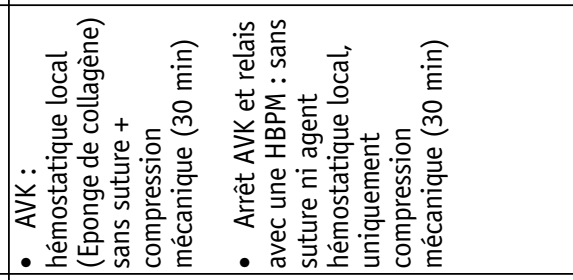 \\
\hline & 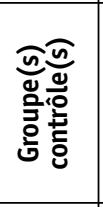 & 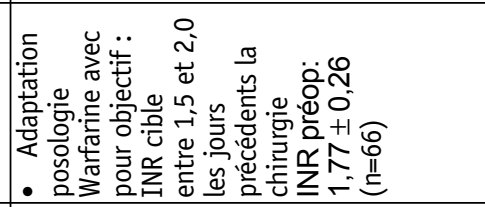 & & 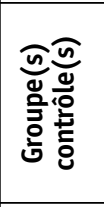 & 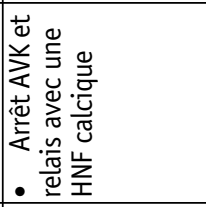 & 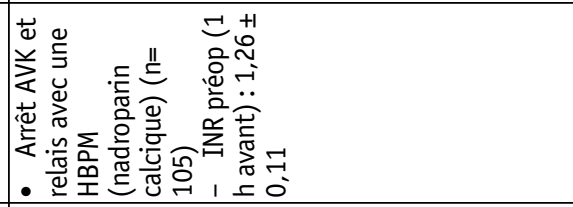 \\
\hline & 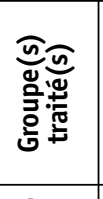 & 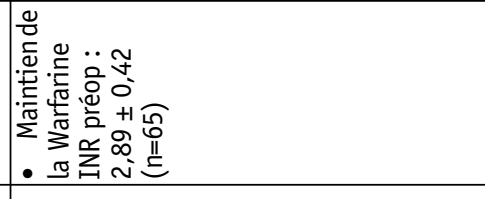 & : & 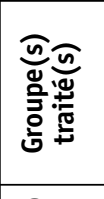 & 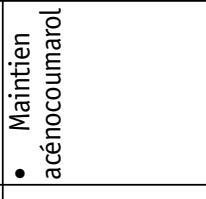 & 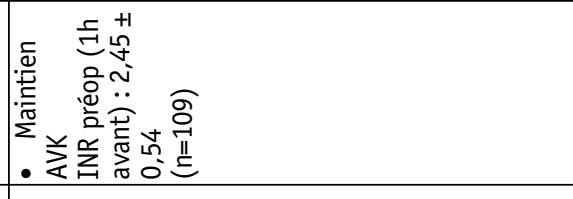 \\
\hline 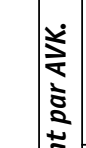 & 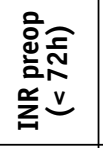 & 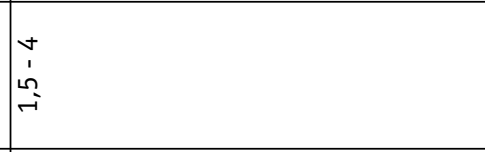 & 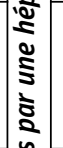 & 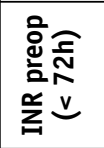 & $\stackrel{n}{\dagger}$ & $\stackrel{v}{v}$ \\
\hline 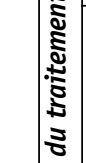 & 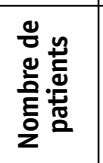 & $\overrightarrow{\vec{m}}$ & 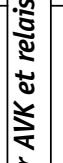 & 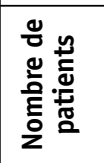 & ను & $\underset{\sim}{\sim}$ \\
\hline 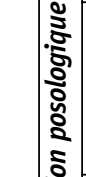 & 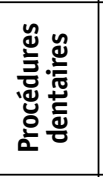 & 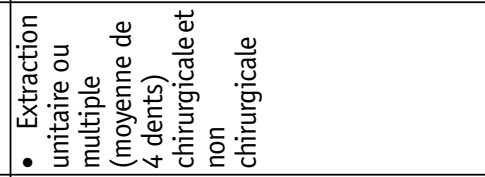 & 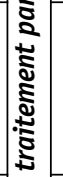 & 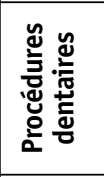 & 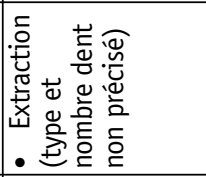 & 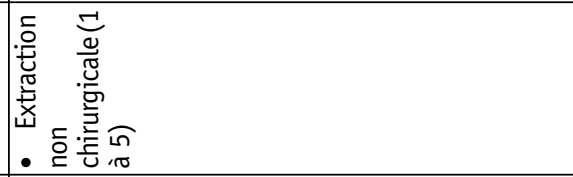 \\
\hline 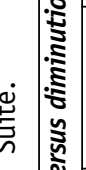 & 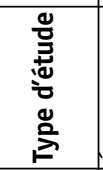 & 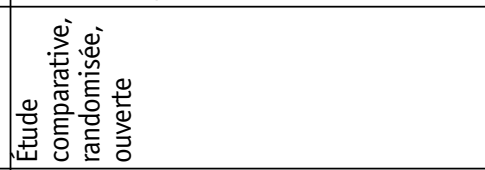 & 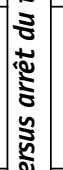 & 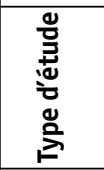 & 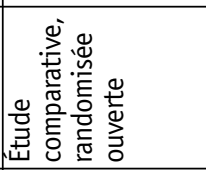 & 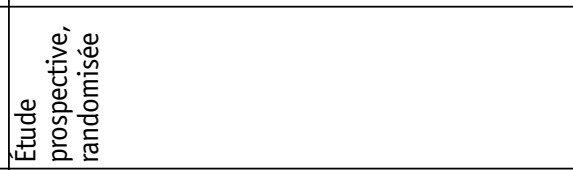 \\
\hline 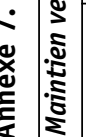 & 离 & 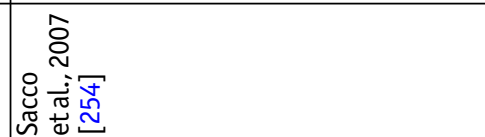 & 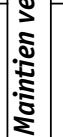 & 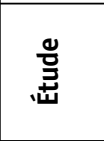 & 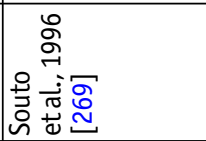 & 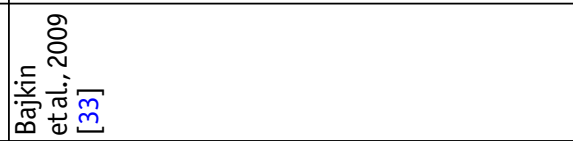 \\
\hline
\end{tabular}




\begin{tabular}{|c|c|c|c|c|}
\hline & 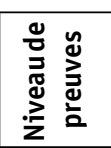 & $r$ & - & $\sim$ \\
\hline & 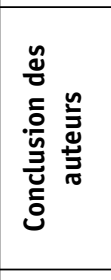 & 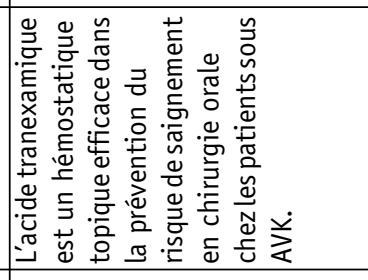 & 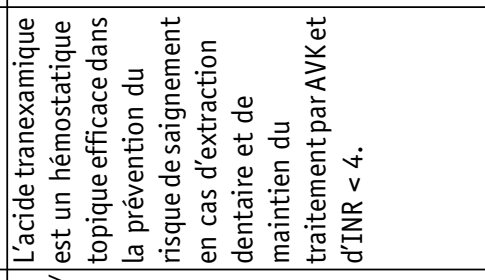 & 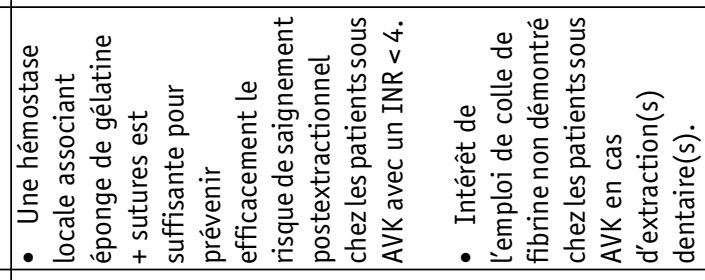 \\
\hline & 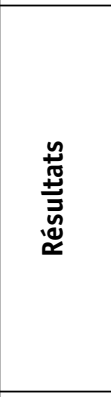 & 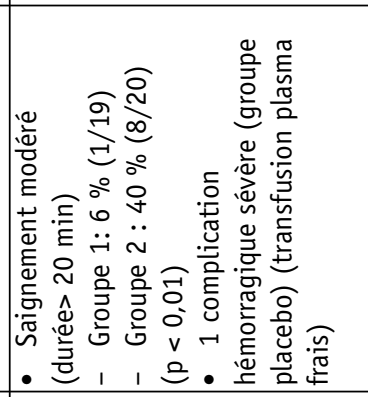 & 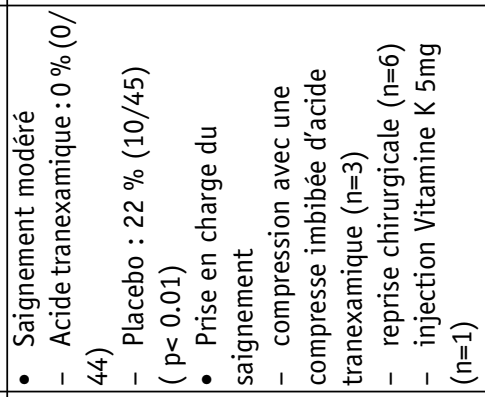 & 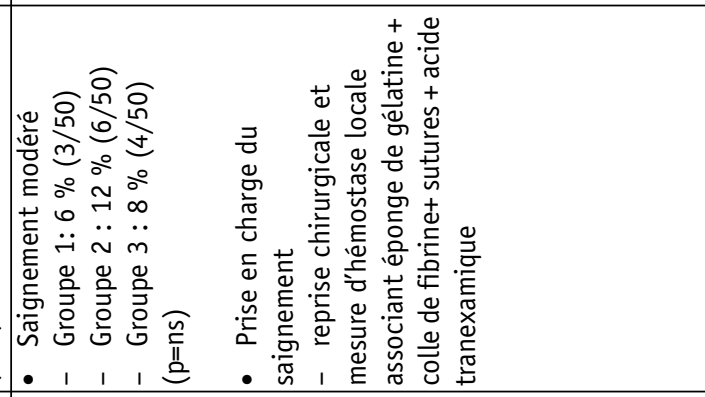 \\
\hline & 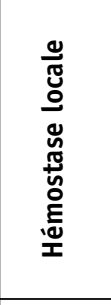 & 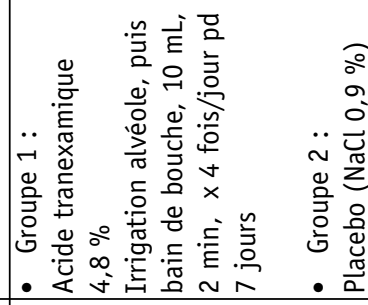 & 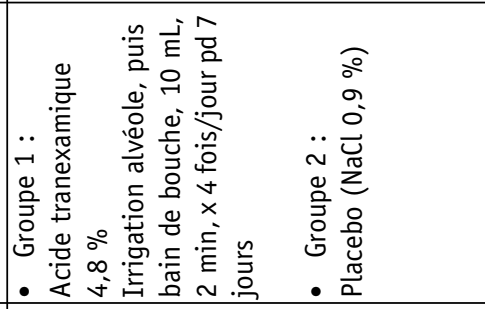 & 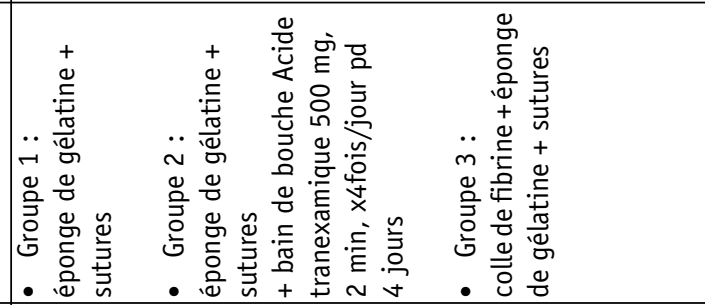 \\
\hline & 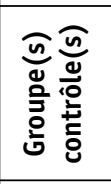 & 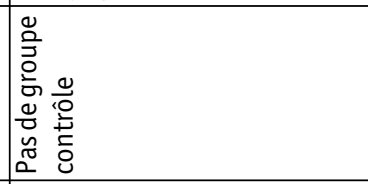 & 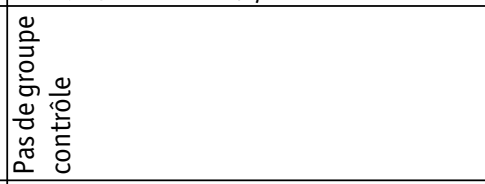 & 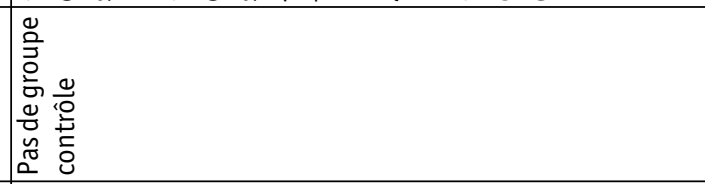 \\
\hline & 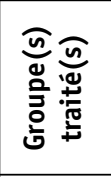 & 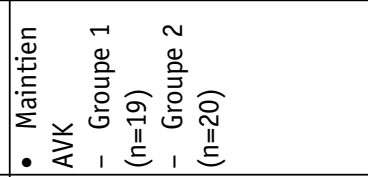 & 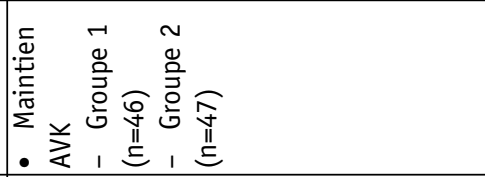 & 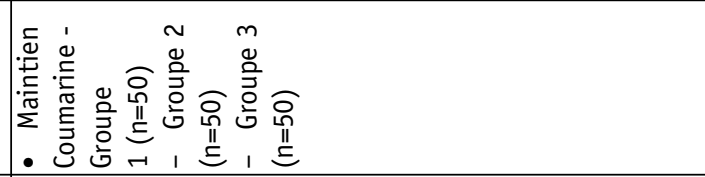 \\
\hline & 总总离 & $=\underset{v}{\stackrel{0}{i}}$ & $\underset{v}{+}$ & 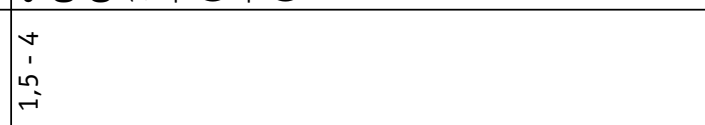 \\
\hline$\frac{\pi}{0}$ & 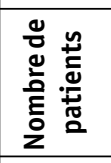 & m) & ৪ & 号 \\
\hline 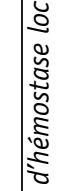 & 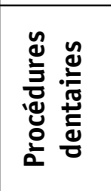 & 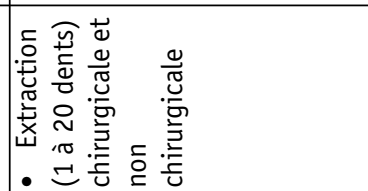 & 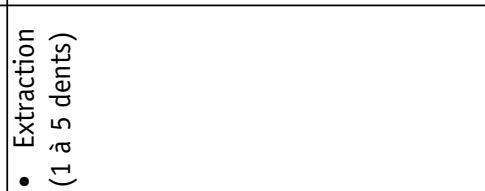 & 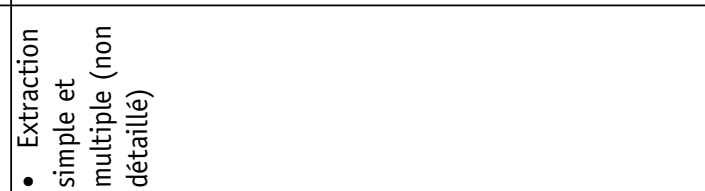 \\
\hline 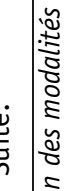 & 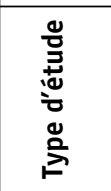 & 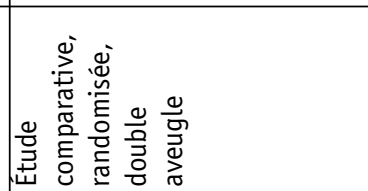 & 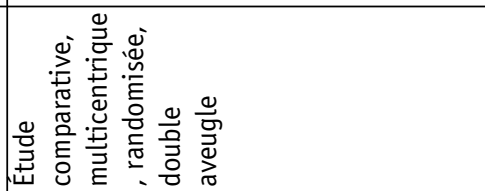 & 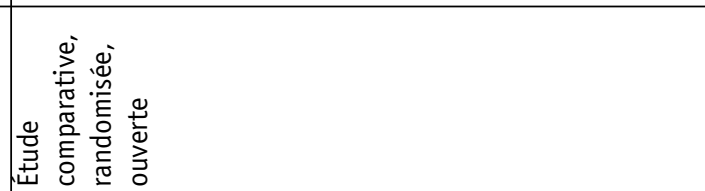 \\
\hline 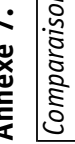 & 壳 & 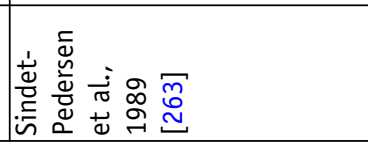 & 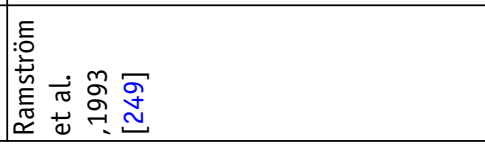 & 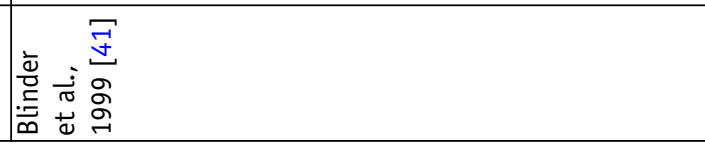 \\
\hline
\end{tabular}




\begin{tabular}{|c|c|c|c|c|c|}
\hline & 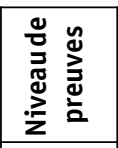 & $\sim$ & $\checkmark$ & $\sim$ & $\sim$ \\
\hline & 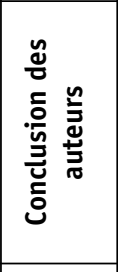 & 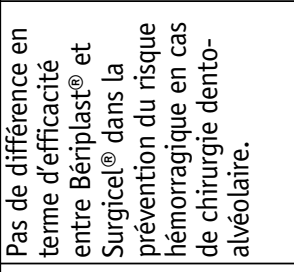 & 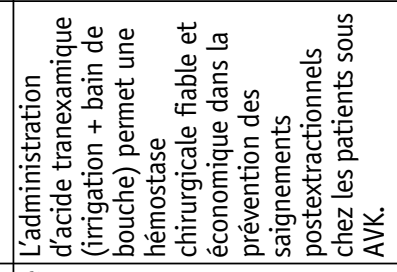 & 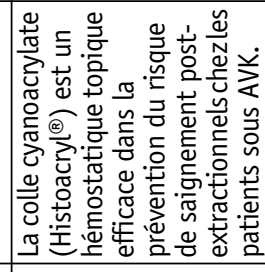 & 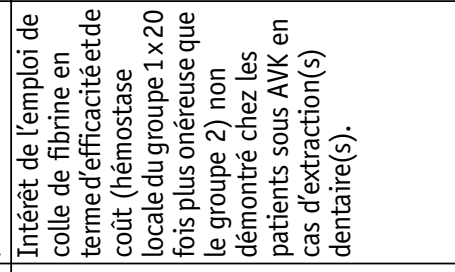 \\
\hline & 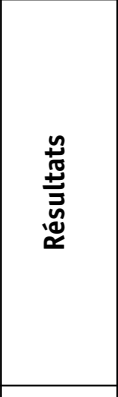 & 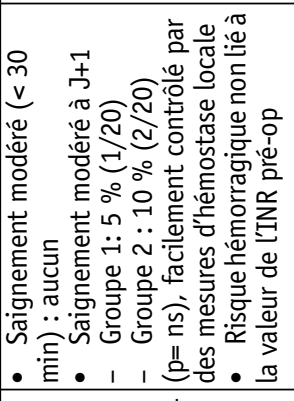 & 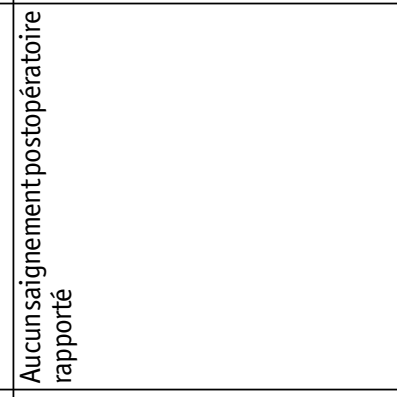 & 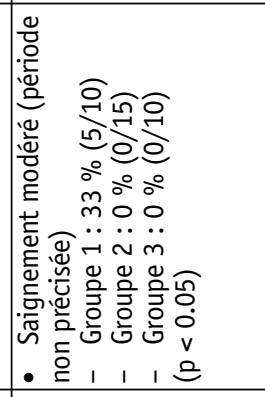 & 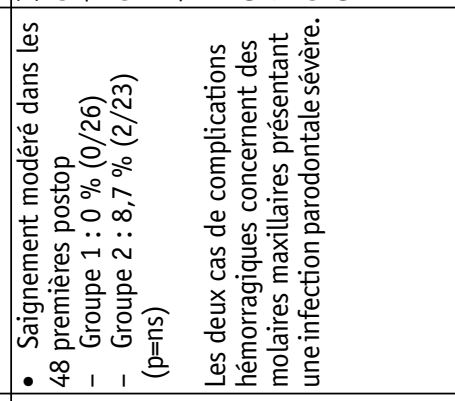 \\
\hline & 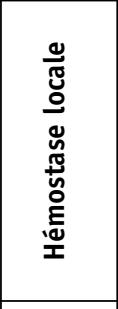 & 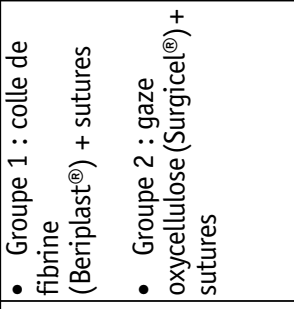 & 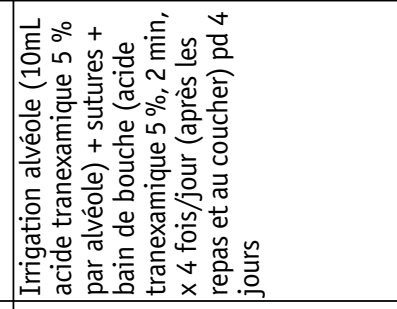 & 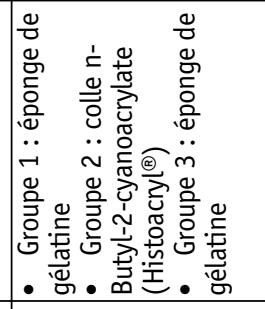 & 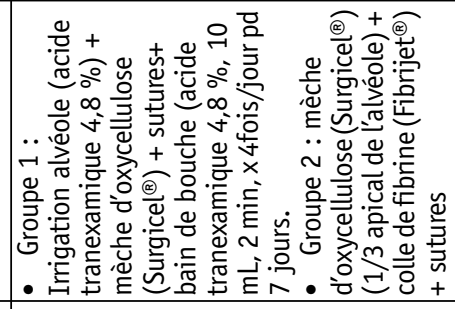 \\
\hline & 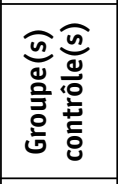 & 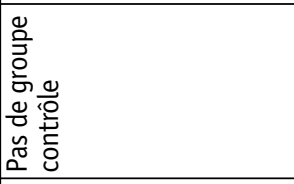 & 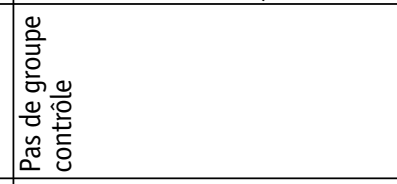 & 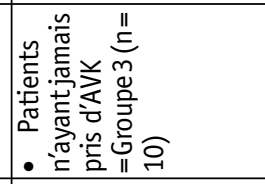 & 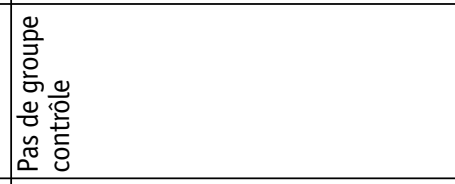 \\
\hline & 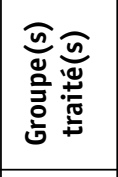 & 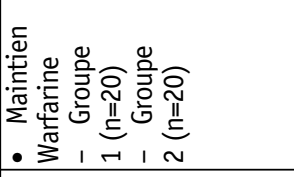 & 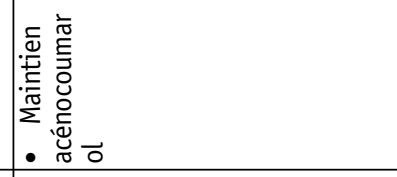 & 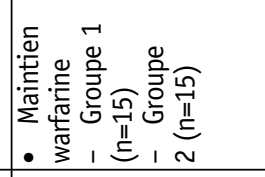 & 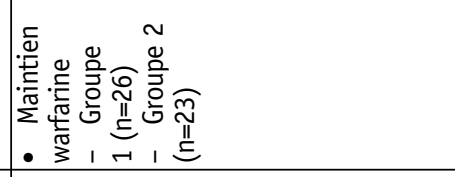 \\
\hline & 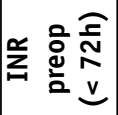 & $\underset{\sim}{\sim}$ & $\stackrel{\dot{y}}{\dot{\nu}}$ & $\begin{array}{l}+ \\
r \\
r\end{array}$ & \begin{tabular}{|l}
$\dot{y}$ \\
$\dot{u}$ \\
$\sim$
\end{tabular} \\
\hline ఫ్ & 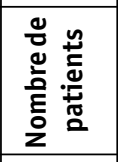 & i̊n & 아 & of & of \\
\hline 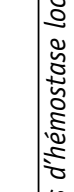 & 过 & 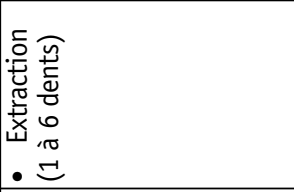 & 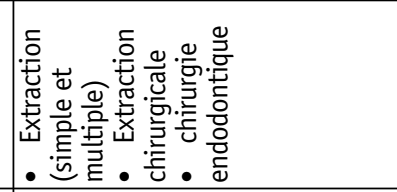 & 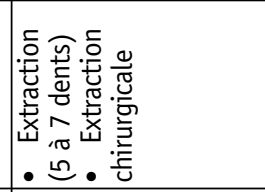 & 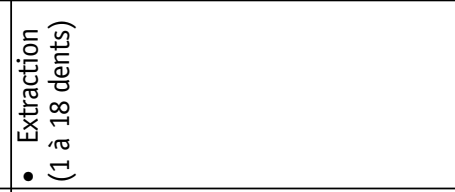 \\
\hline 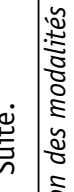 & 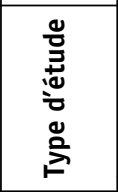 & 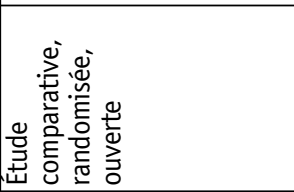 & 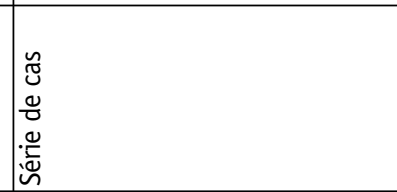 & 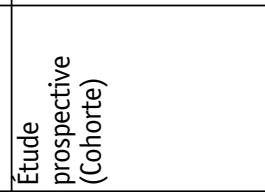 & 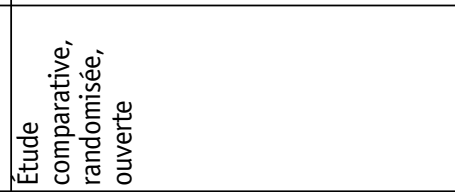 \\
\hline 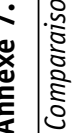 & 莹 & 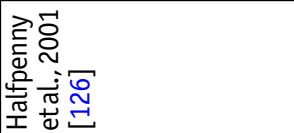 & 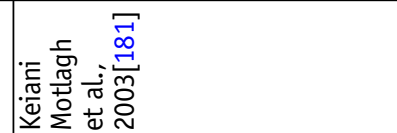 & 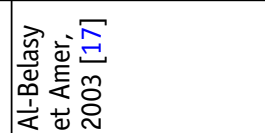 & 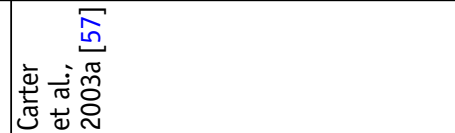 \\
\hline
\end{tabular}




\begin{tabular}{|c|c|c|c|}
\hline & 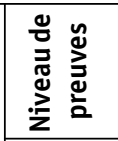 & $\vec{r}$ & $\sim$ \\
\hline & 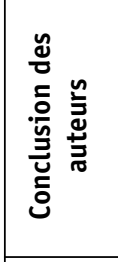 & 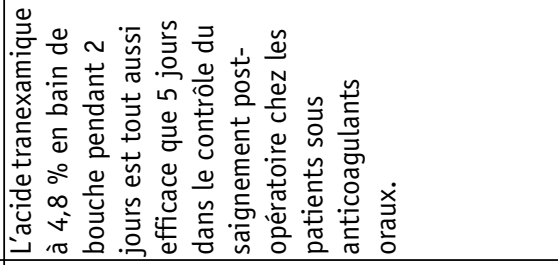 & 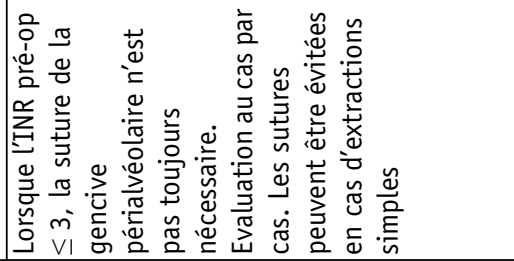 \\
\hline & 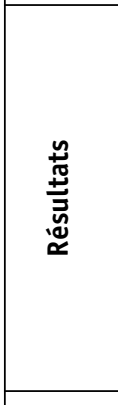 & 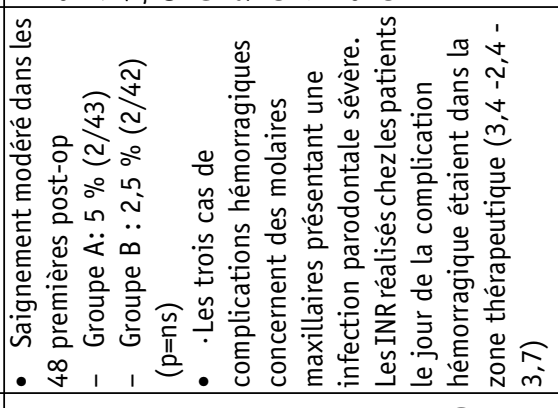 & 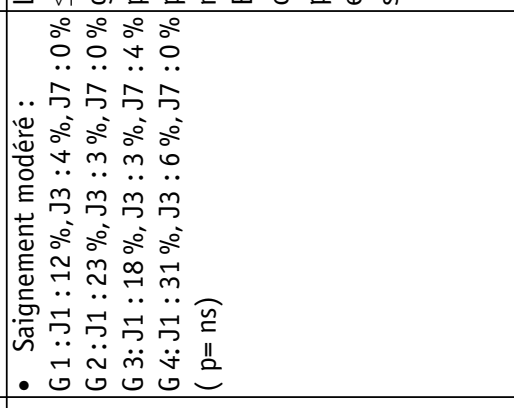 \\
\hline & 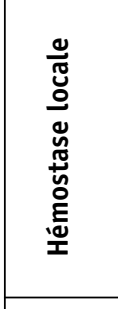 & 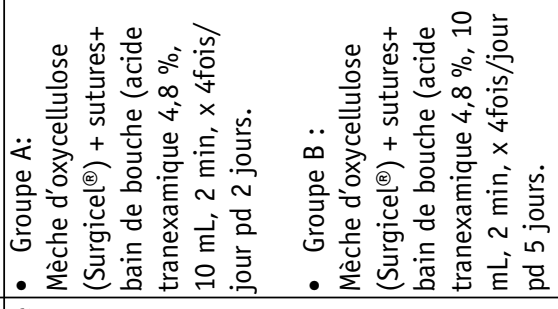 & 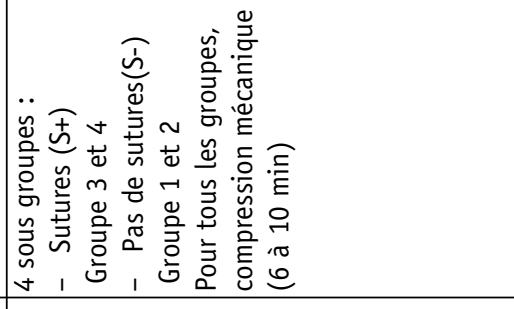 \\
\hline & 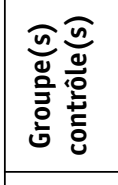 & 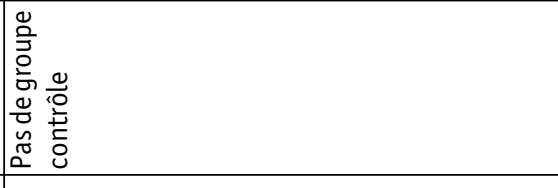 & 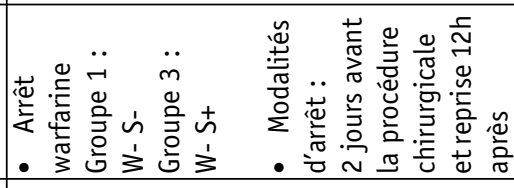 \\
\hline & 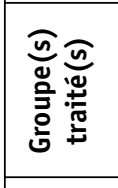 & 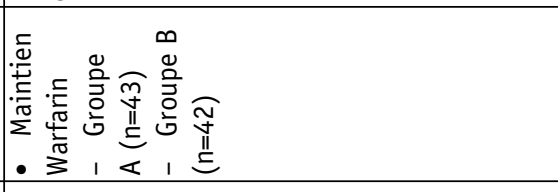 & 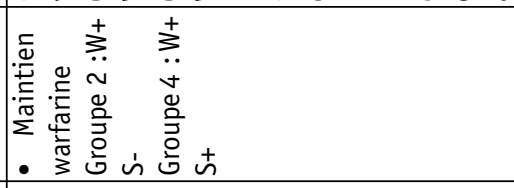 \\
\hline & 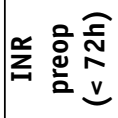 & $\begin{array}{l}\text { a } \\
\dot{1} \\
\sim\end{array}$ & 年 \\
\hline$\frac{\mathbb{Z}}{\tilde{Z}}$ & 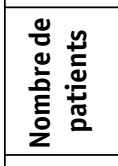 & $\infty$ & $\underset{\sim}{\mathbb{N}}$ \\
\hline 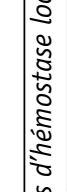 & 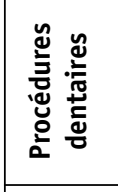 & 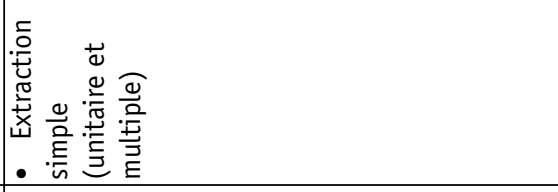 & 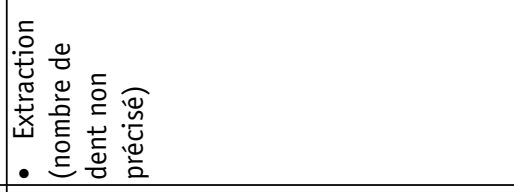 \\
\hline 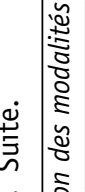 & 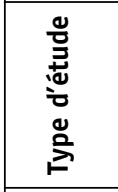 & 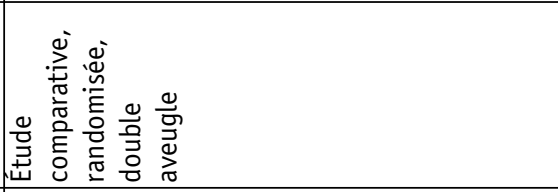 & 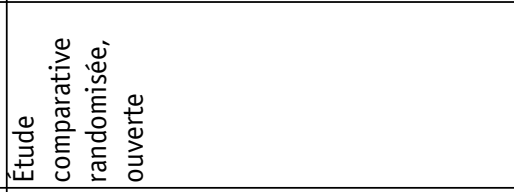 \\
\hline $\mid$ & 莡 & 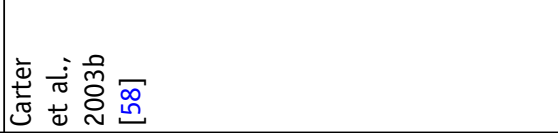 & 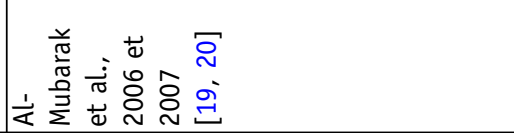 \\
\hline
\end{tabular}




\begin{tabular}{|c|c|c|c|c|c|c|}
\hline & & 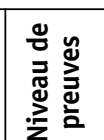 & $\checkmark$ & $\sim$ & $\checkmark$ & $\sim$ \\
\hline & & 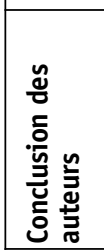 & 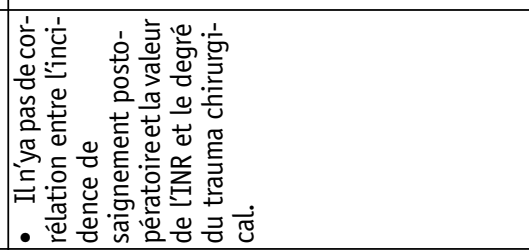 & 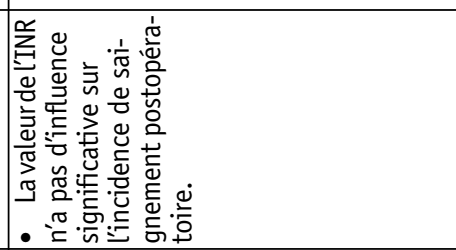 & 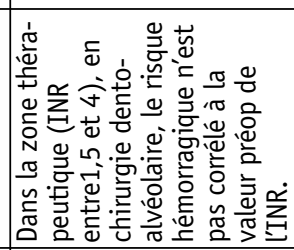 & 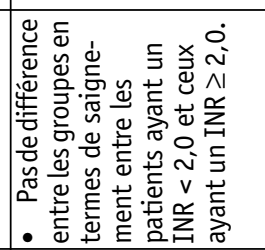 \\
\hline & & 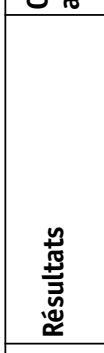 & 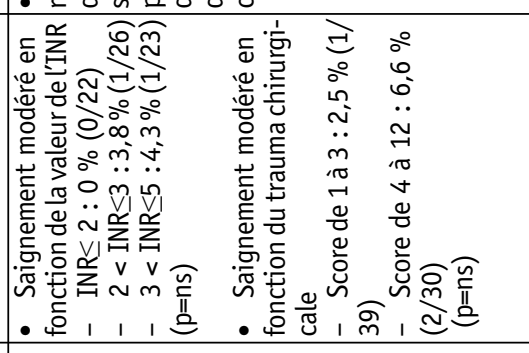 & 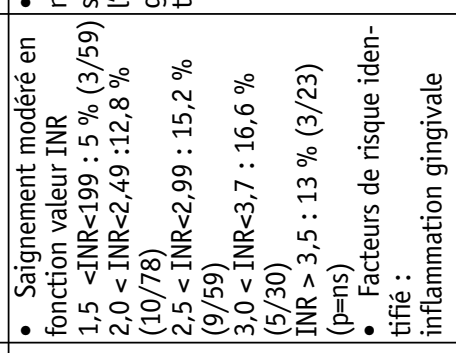 & 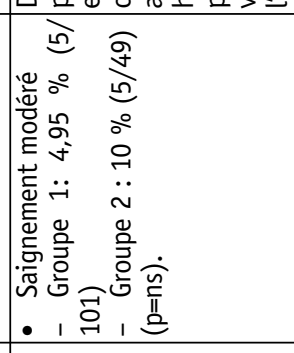 & 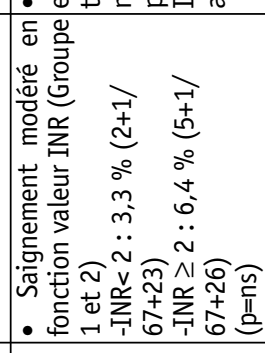 \\
\hline & & 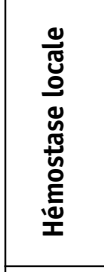 & 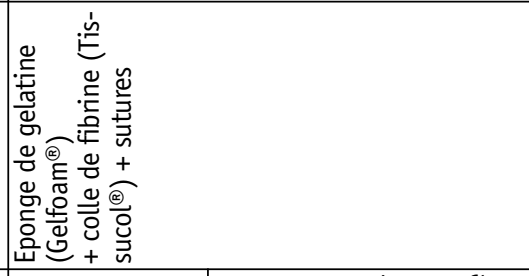 & 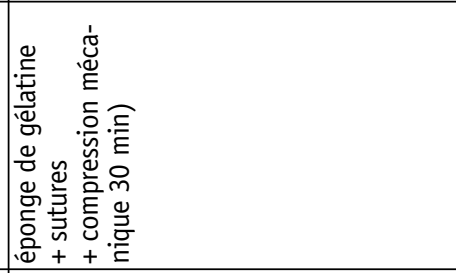 & 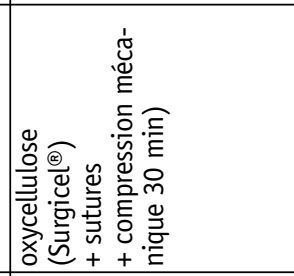 & 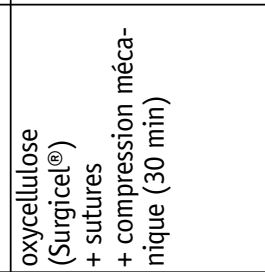 \\
\hline 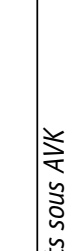 & & 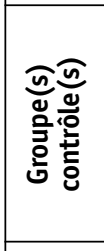 & 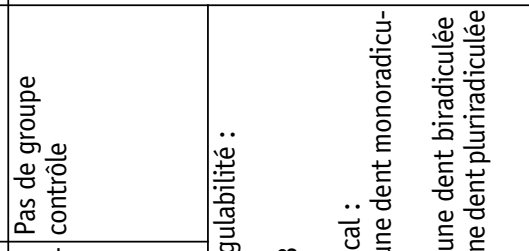 & 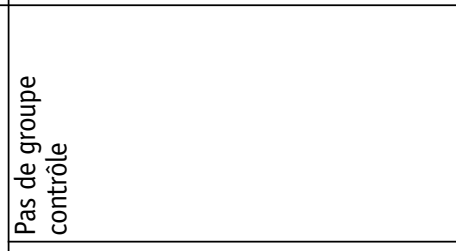 & 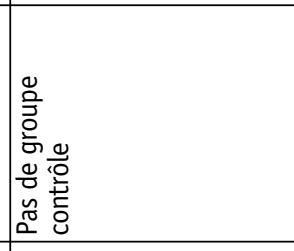 & 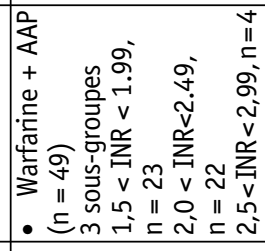 \\
\hline 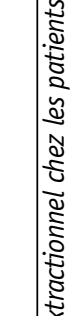 & & 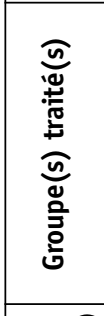 & 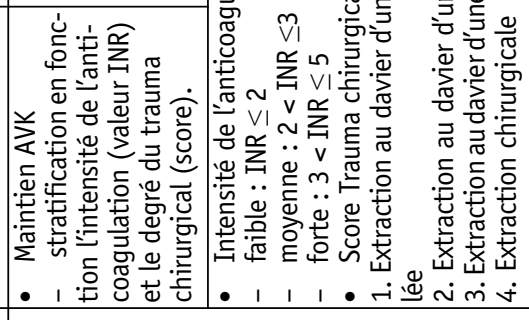 & 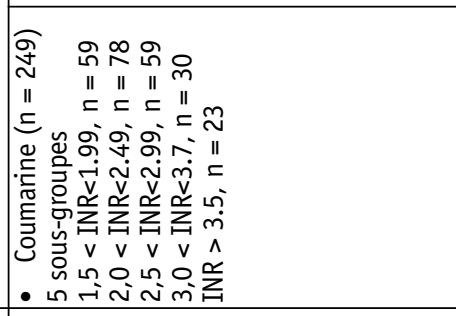 & 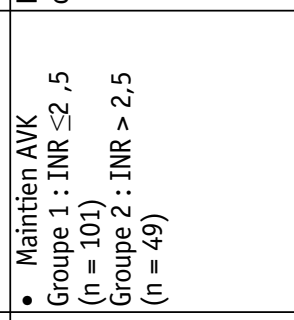 & 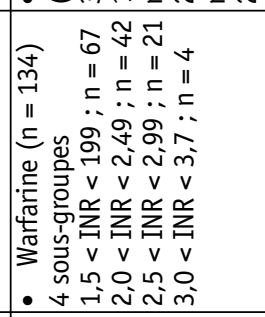 \\
\hline 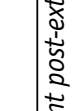 & & 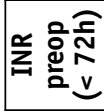 & n & & $\underset{v}{\checkmark}$ & 常 \\
\hline 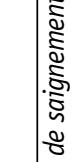 & & 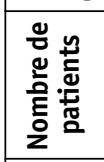 & 8 & $\underset{\sim}{\text { Q }}$ & 号 & $\underset{N}{\stackrel{R}{N}}$ \\
\hline 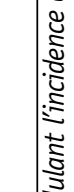 & 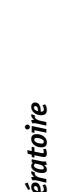 & 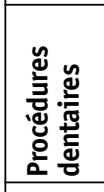 & 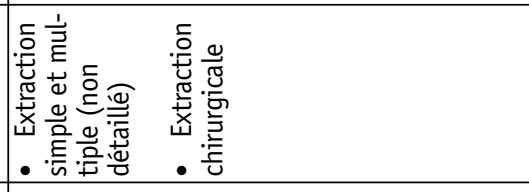 & 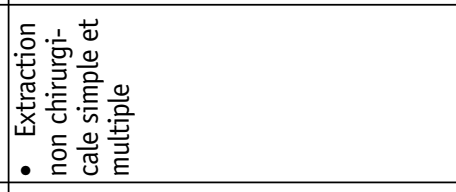 & 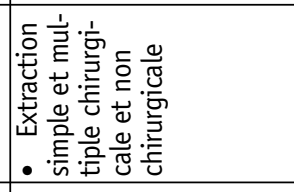 & 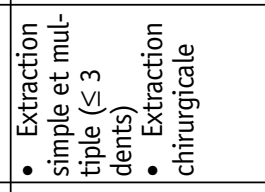 \\
\hline 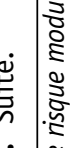 & 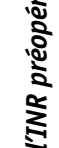 & 总蒂 & 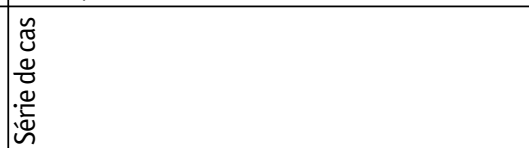 & 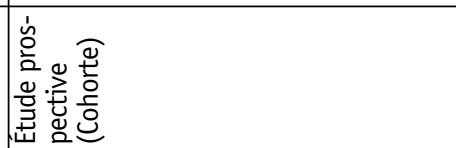 & 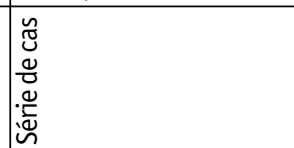 & 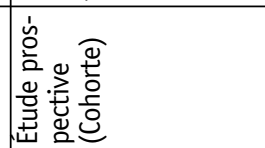 \\
\hline 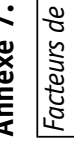 & 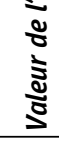 & $\stackrel{\text { D্ }}{\mathrm{Z}}$ & 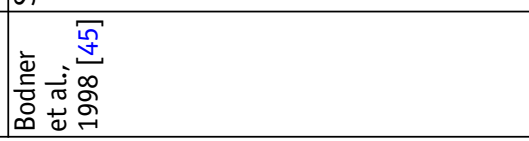 & 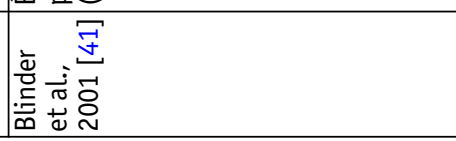 & 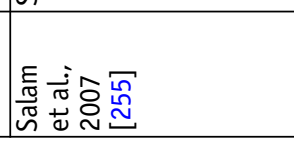 & 尊 \\
\hline
\end{tabular}



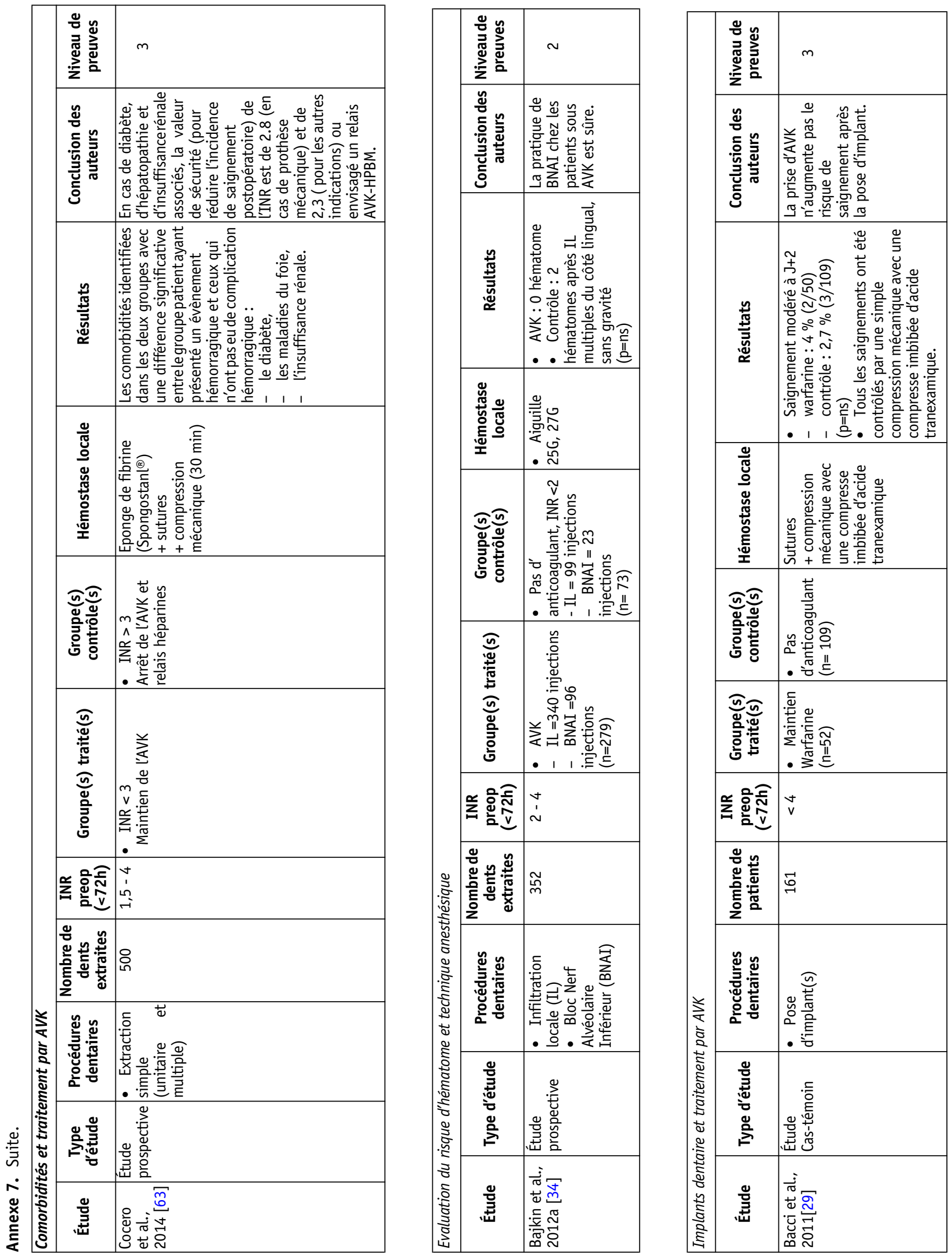


\begin{tabular}{|c|c|c|c|c|}
\hline & 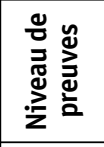 & $\sim$ & $\sim$ & $\sim$ \\
\hline & 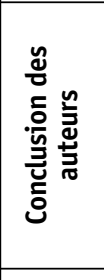 & 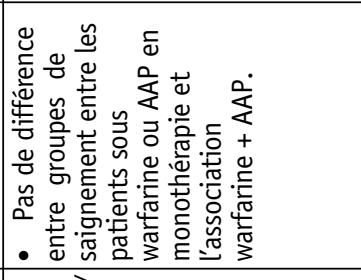 & 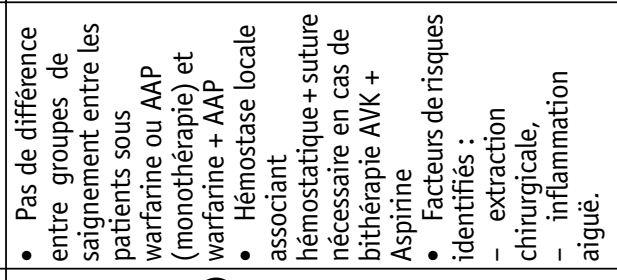 & 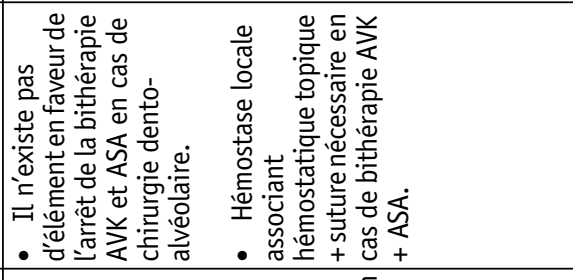 \\
\hline & 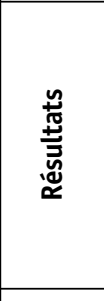 & 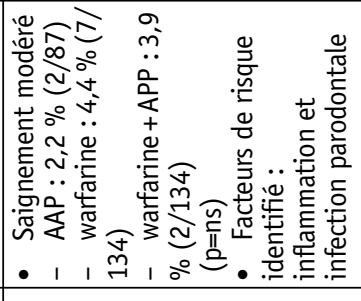 & 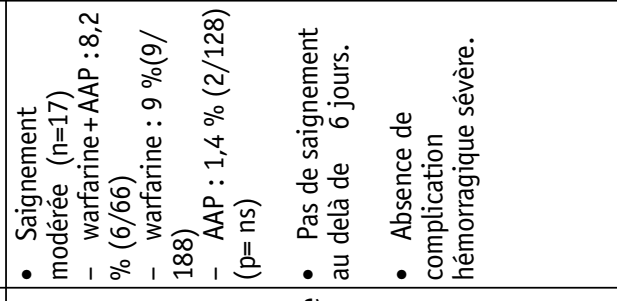 & 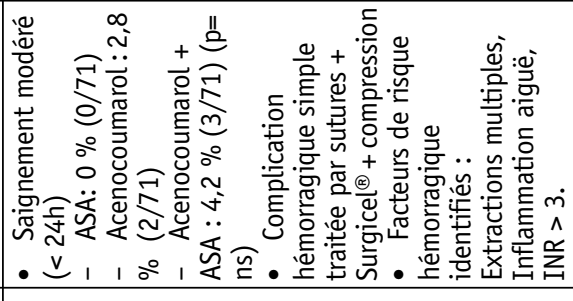 \\
\hline & 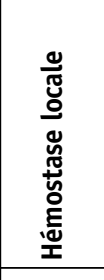 & 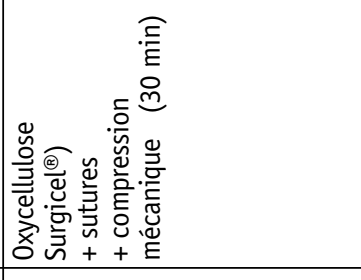 & 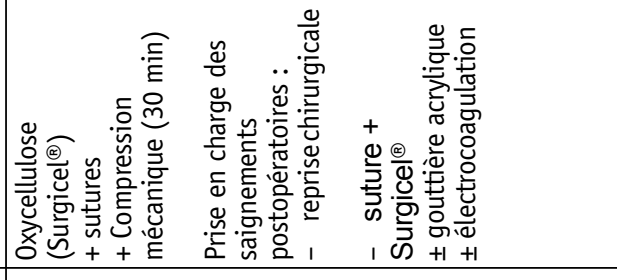 & 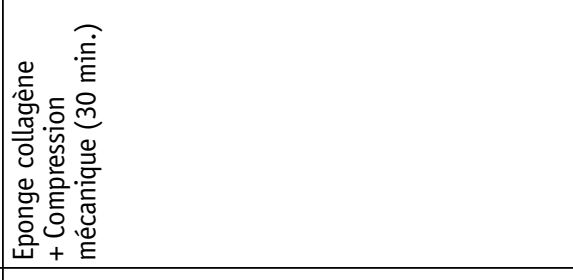 \\
\hline & 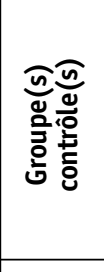 & 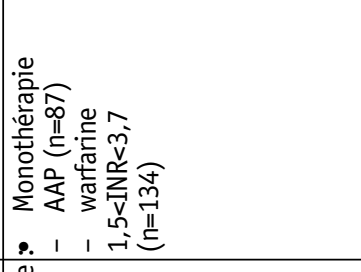 & 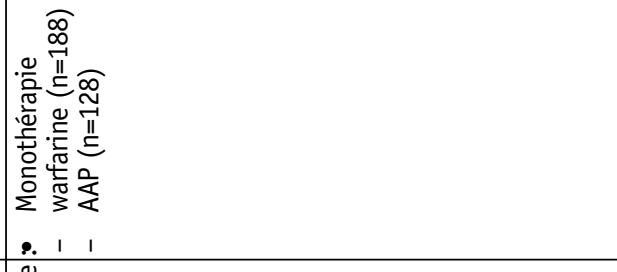 & 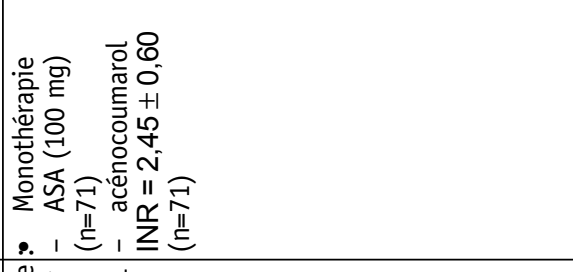 \\
\hline & 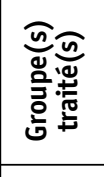 & 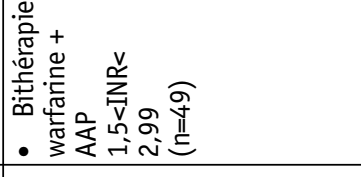 & 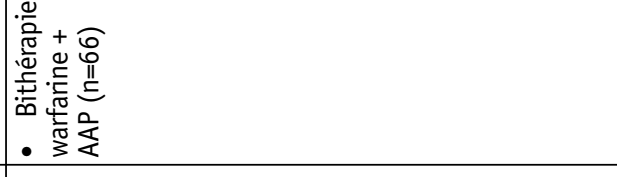 & 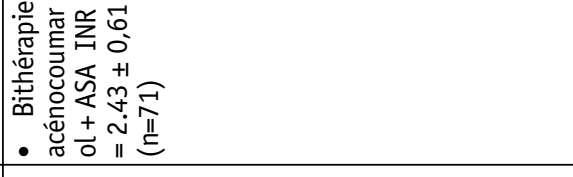 \\
\hline & 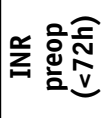 & 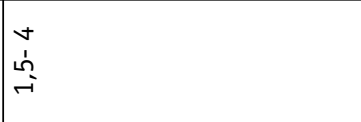 & 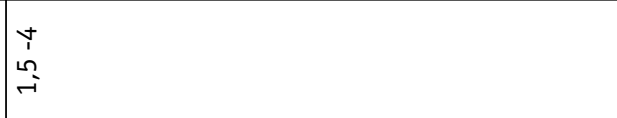 & $\begin{array}{l}+ \\
\stackrel{1}{\prime}\end{array}$ \\
\hline & 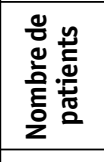 & $\stackrel{?}{\text { న }}$ & 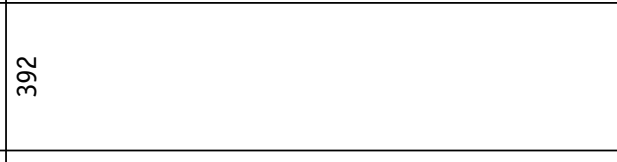 & $\stackrel{m}{\sim}$ \\
\hline & 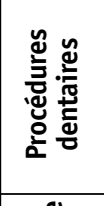 & 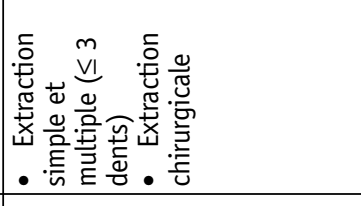 & 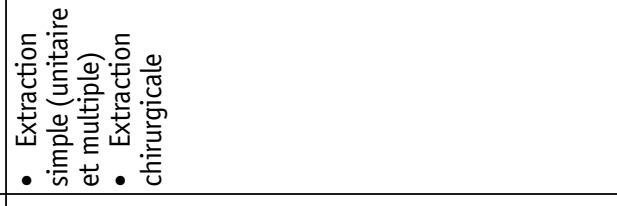 & 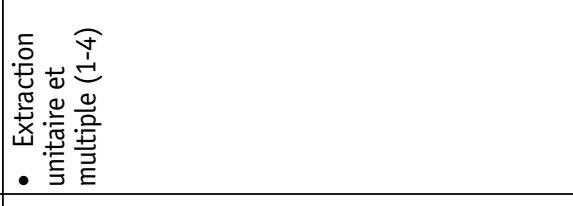 \\
\hline $\begin{array}{l}\vdots \\
\vdots \\
+ \\
\vdots\end{array}$ & 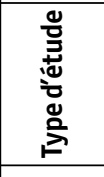 & 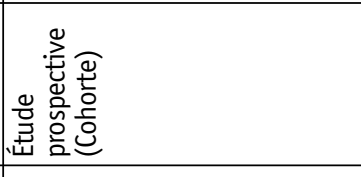 & 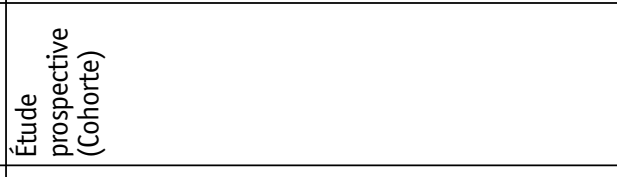 & 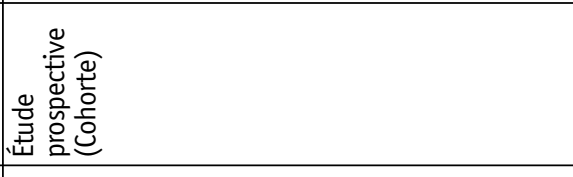 \\
\hline 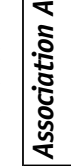 & 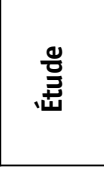 & 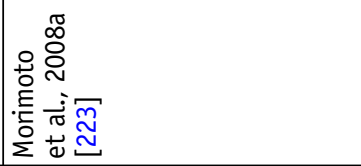 & 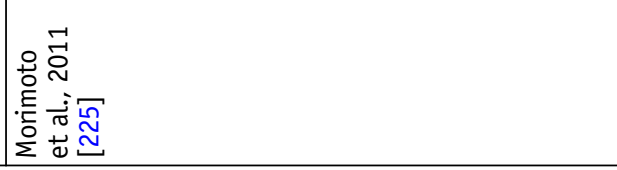 & 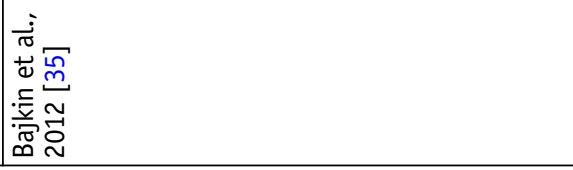 \\
\hline
\end{tabular}


Annexe 9. Protocole d'arrêt et de reprise d'un AVK sans relais héparinique pour une chirurgie à risque hémorragique élevé chez un patient à risque thrombotique faible.

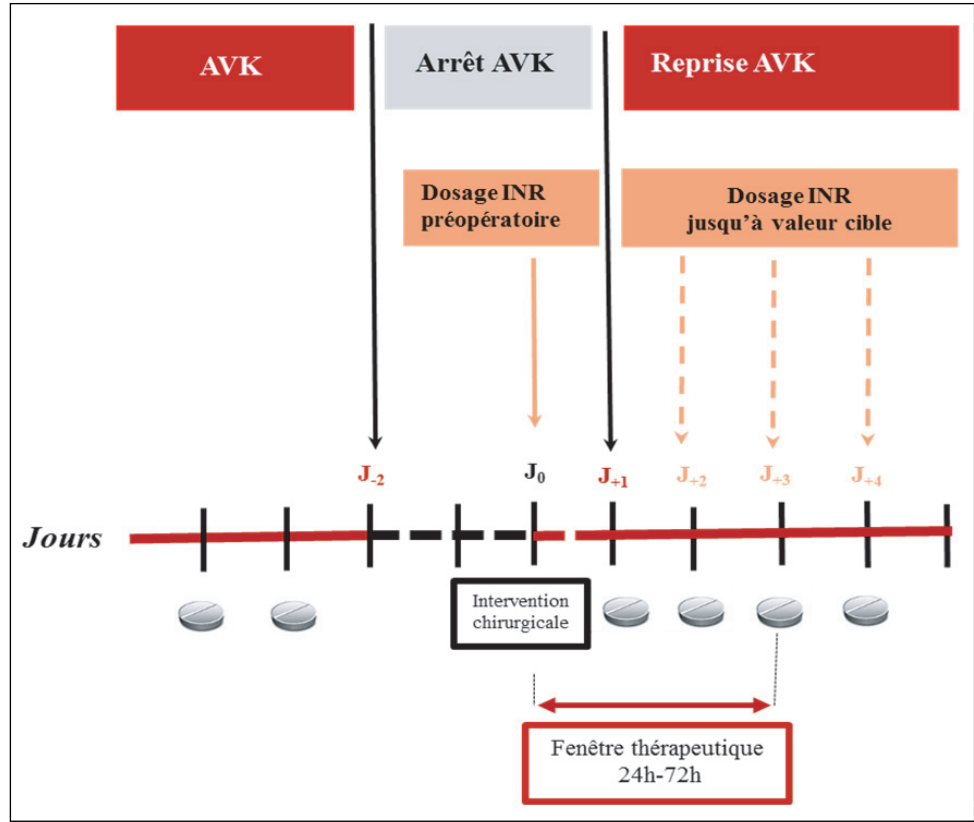

Annexe 10. Protocole d'arrêt et de reprise d'un AVK avec un relais héparinique pour une chirurgie à risque hémorragique élevé chez un patient à risque thrombotique élevé.

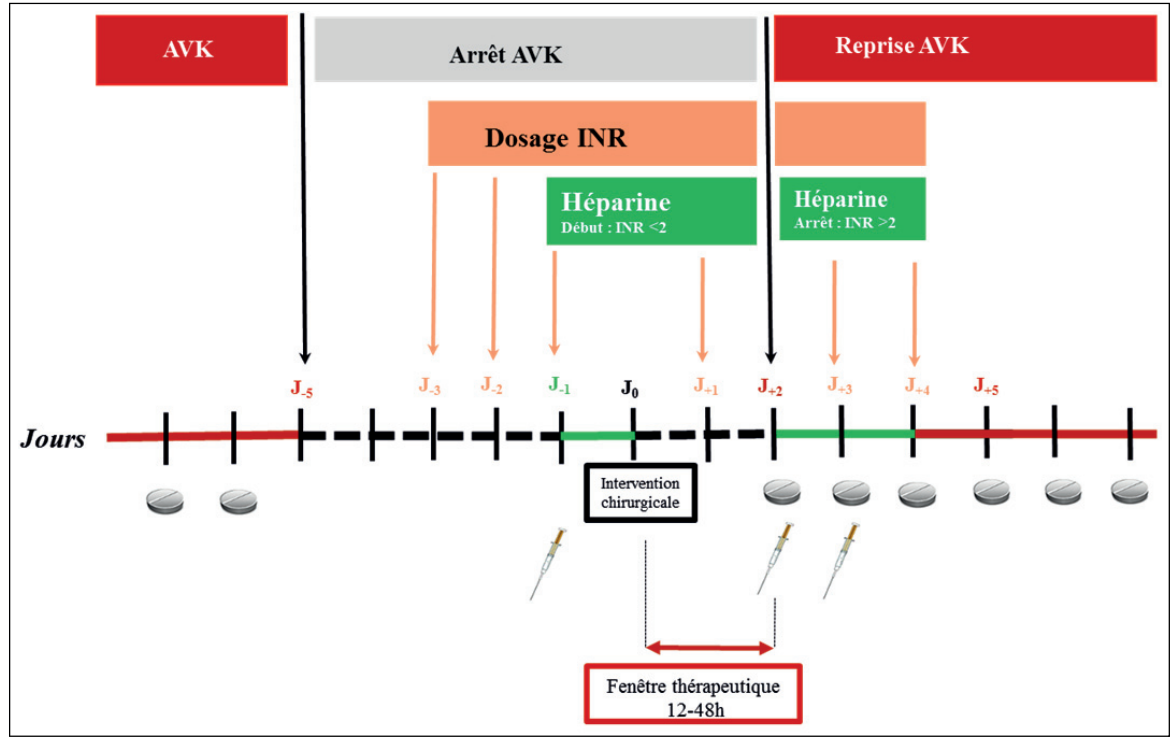

Cette stratégie thérapeutique ne s'applique qu'en cas de chirurgie programmée.

1. Arrêt des AVK 5 jours avant la procédure l'intervention chirurgicale.

2. Contrôle de l'INR, tous les 24-48h.

3. Le traitement par l'héparine est débuté dès que l'INR est inférieur à 2, à raison de 1 à 2 injection(s) par jour en fonction du risque thrombo-embolique.

4. L'intervention chirurgicale est programmée. Arrêt de l'héparine $12 \mathrm{~h}$ avant l'acte chirurgical. En pratique, l'injection du matin est annulée. 5. Reprise dès que possible de l'héparine et de l'AVK en fonction du contrôle hémostatique. En pratique, reprise de l'héparine et de l'AVK à J+1 ou J+2.

6. Contrôle de l'INR tous les deux jours, arrêt de l'héparine dès que l'INR cible est atteint. 
Annexe 11. Principaux paramètres pharmacocinétiques des AOD

\begin{tabular}{|l|l|l|l|}
\hline Paramètres pharmacocinétiques & \multicolumn{1}{|c|}{ Dabigatran } & \multicolumn{1}{c|}{ Rivaroxaban } & \multicolumn{1}{c|}{ Apixaban } \\
\hline $\mathrm{t}_{1 / 2}$ (demi-vie d'élimination) & $\begin{array}{l}12-14 \mathrm{~h} \\
\text { Prolongée si insuffisance rénale }\end{array}$ & $\begin{array}{l}5-9 \mathrm{~h} \text { chez sujets jeunes } \\
11-13 \mathrm{~h} \text { chez sujets âgés }\end{array}$ & $12 \mathrm{~h}$ \\
\hline $\mathrm{T}_{\max }$ & $0,5-2 \mathrm{~h}$ après la prise & $2-4 \mathrm{~h}$ après la prise & $3-4 \mathrm{~h}$ après la prise \\
\hline $\mathrm{T}_{\min }$ & $12-24 \mathrm{~h}$ après la prise & $16-24 \mathrm{~h}$ après la prise & $12-24 \mathrm{~h}$ après la prise \\
\hline $\begin{array}{l}\text { Principaux facteurs d'augmentation de } \\
\text { l'aire sous la courbe }\end{array}$ & $\begin{array}{l}\text { Insuffisance rénale } \\
\hat{A} g e \\
\text { Petit poids }\end{array}$ & $\begin{array}{l}\text { Insuffisance rénale } \\
\hat{A} g e \\
\text { Insuffisance hépatique }\end{array}$ & $\begin{array}{l}\text { Insuffisance rénale } \\
\hat{A} g e \\
\text { Petit poids }\end{array}$ \\
\hline
\end{tabular}

Annexe 12. Protocole d'arrêt et de reprise d'un AOD sans relais héparinique pour une chirurgie à risque hémorragique élevé.

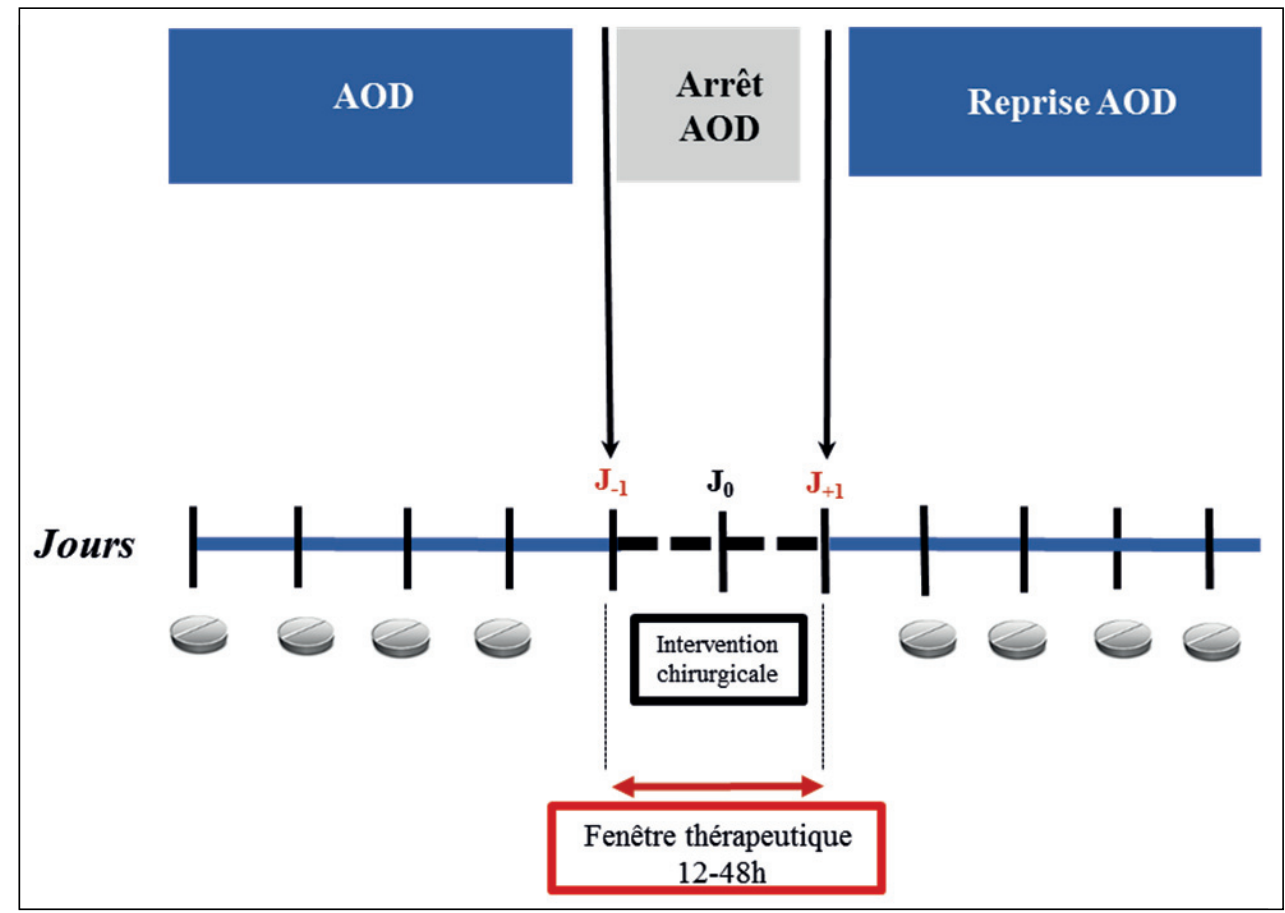




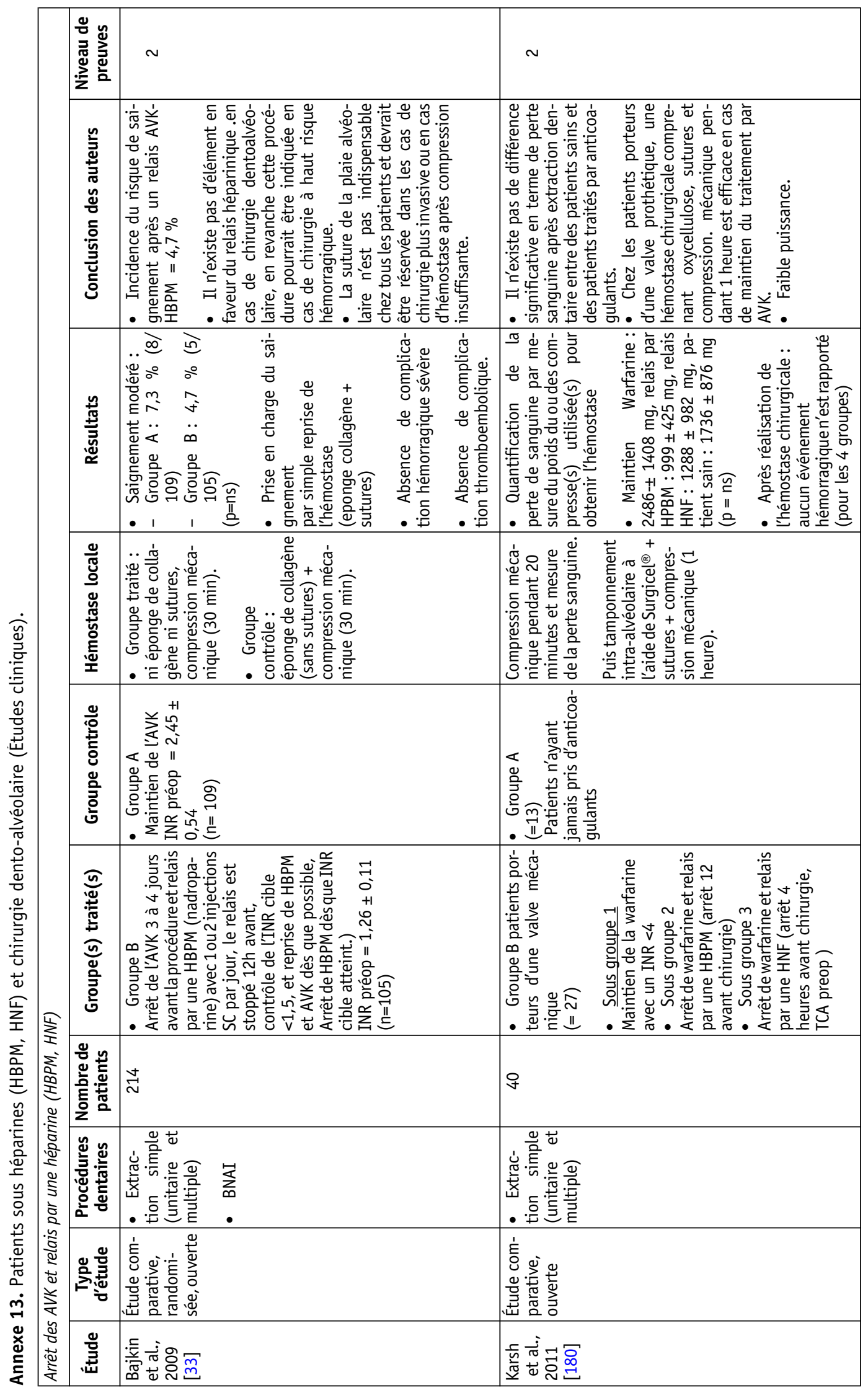




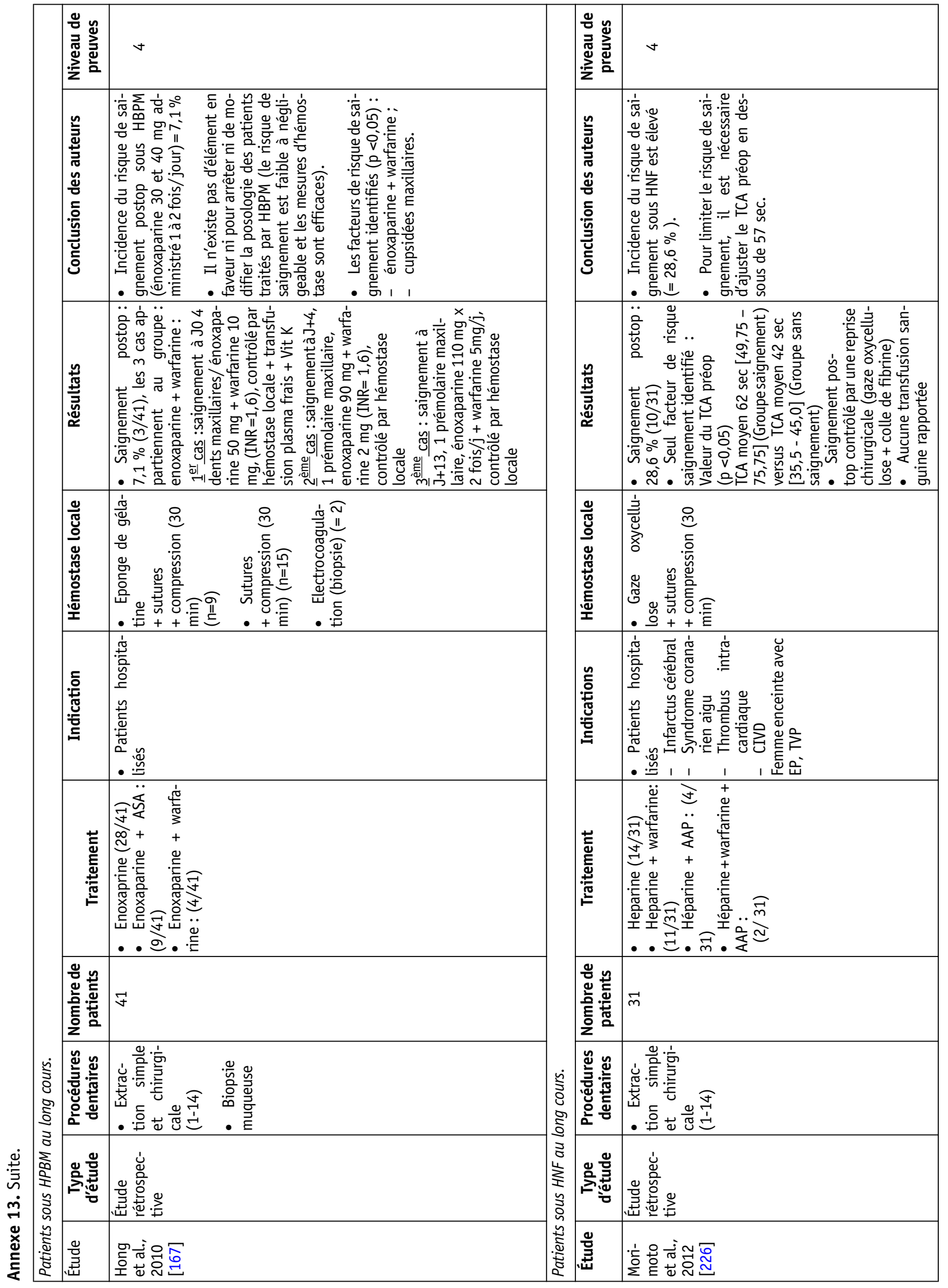




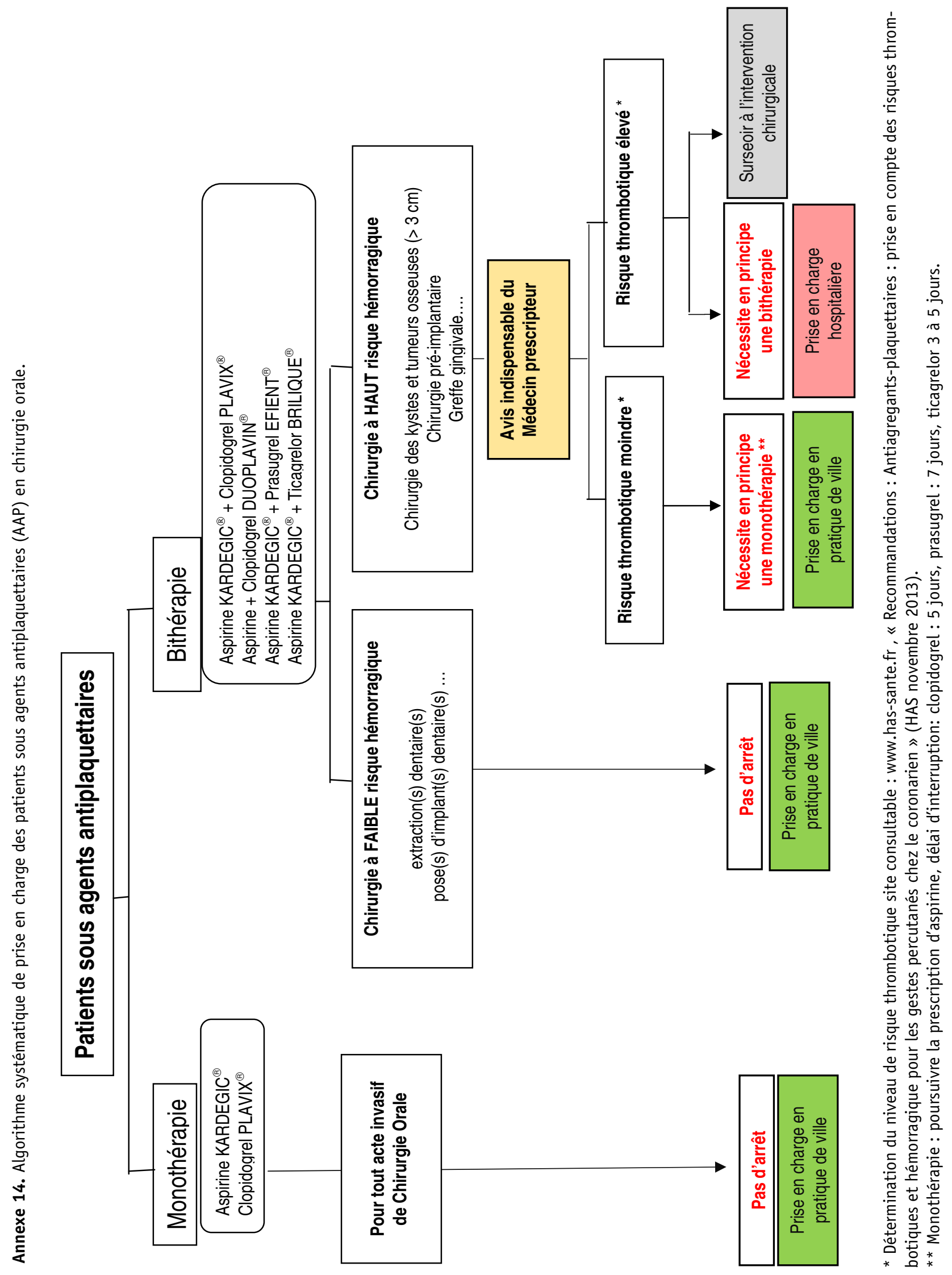




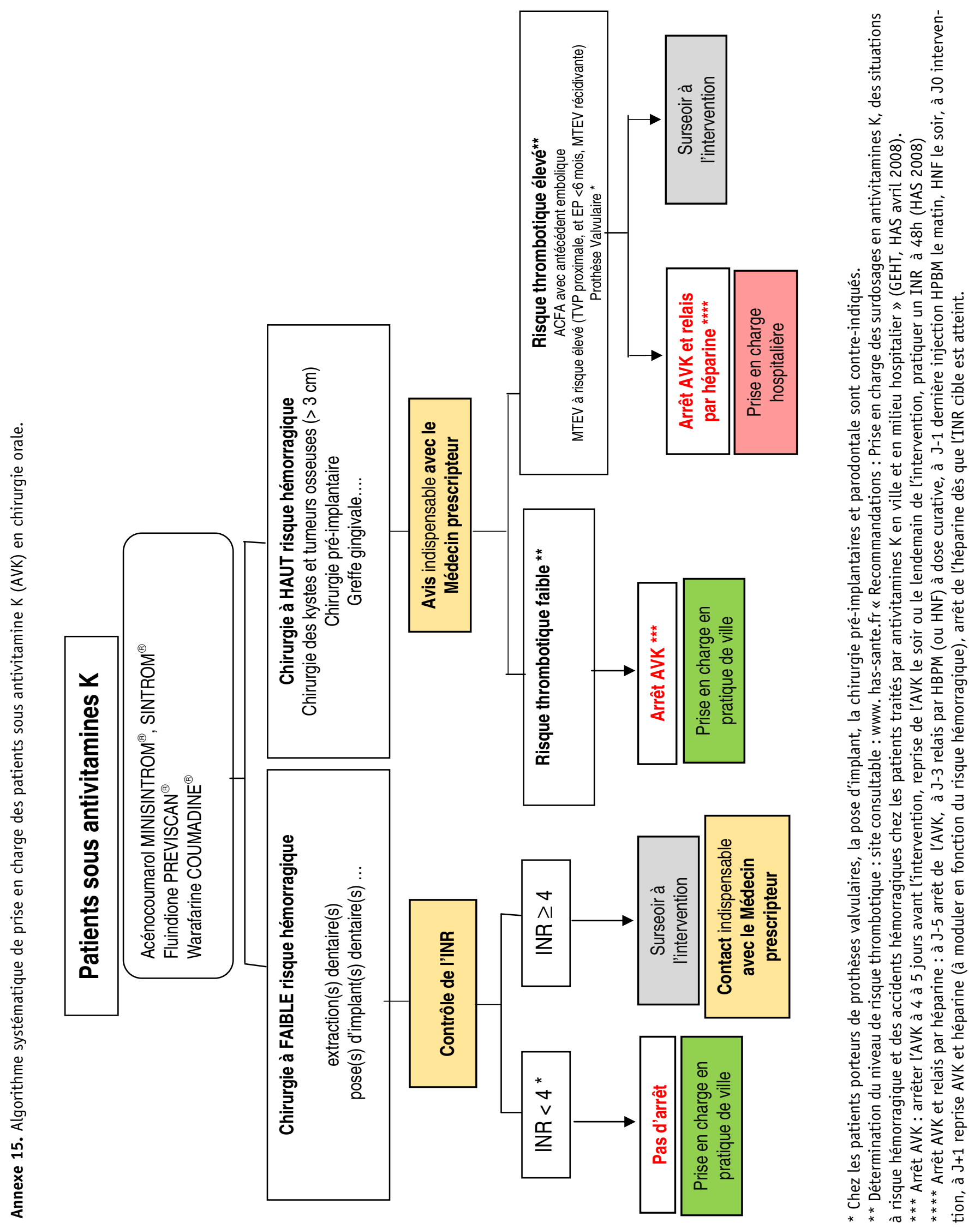




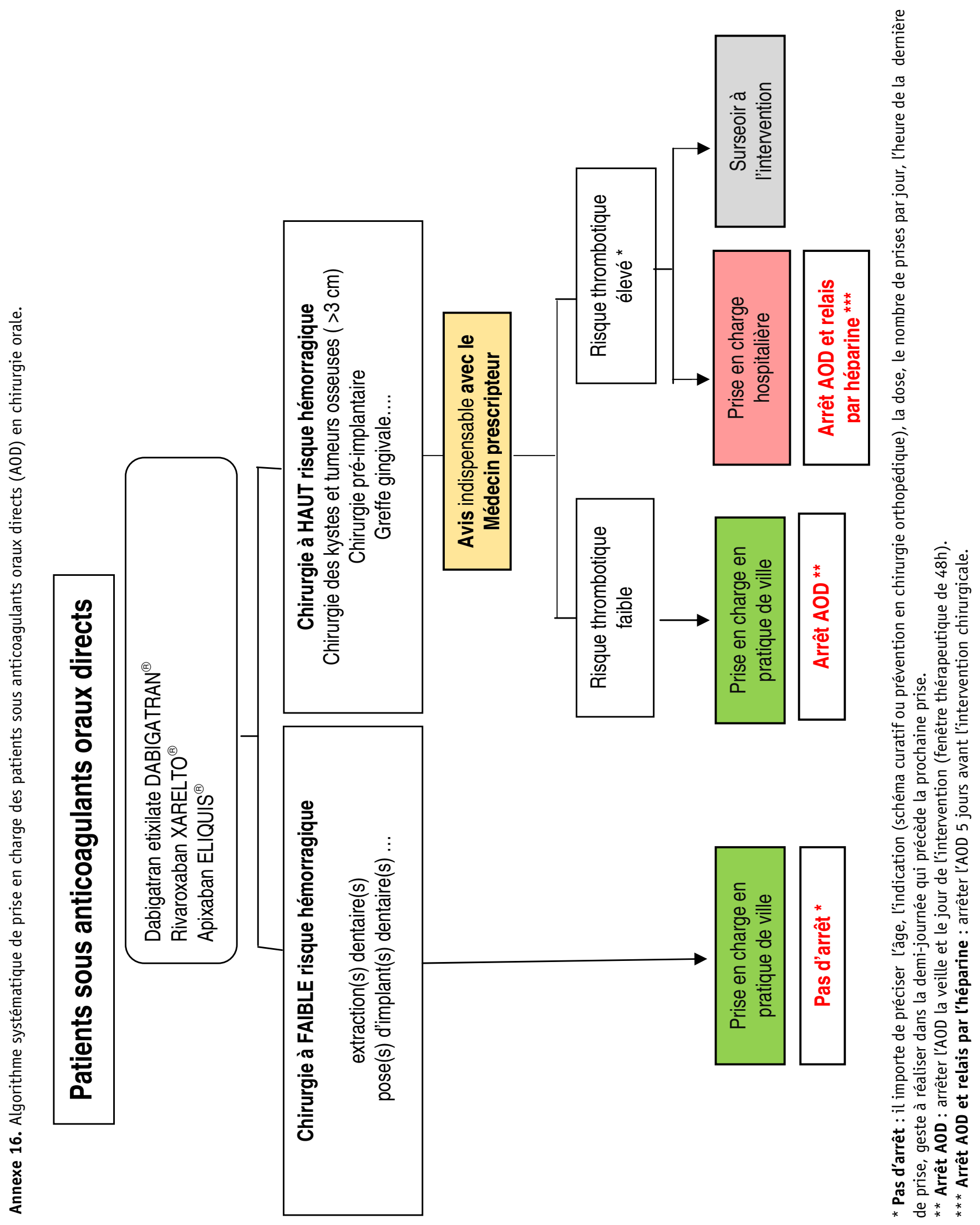




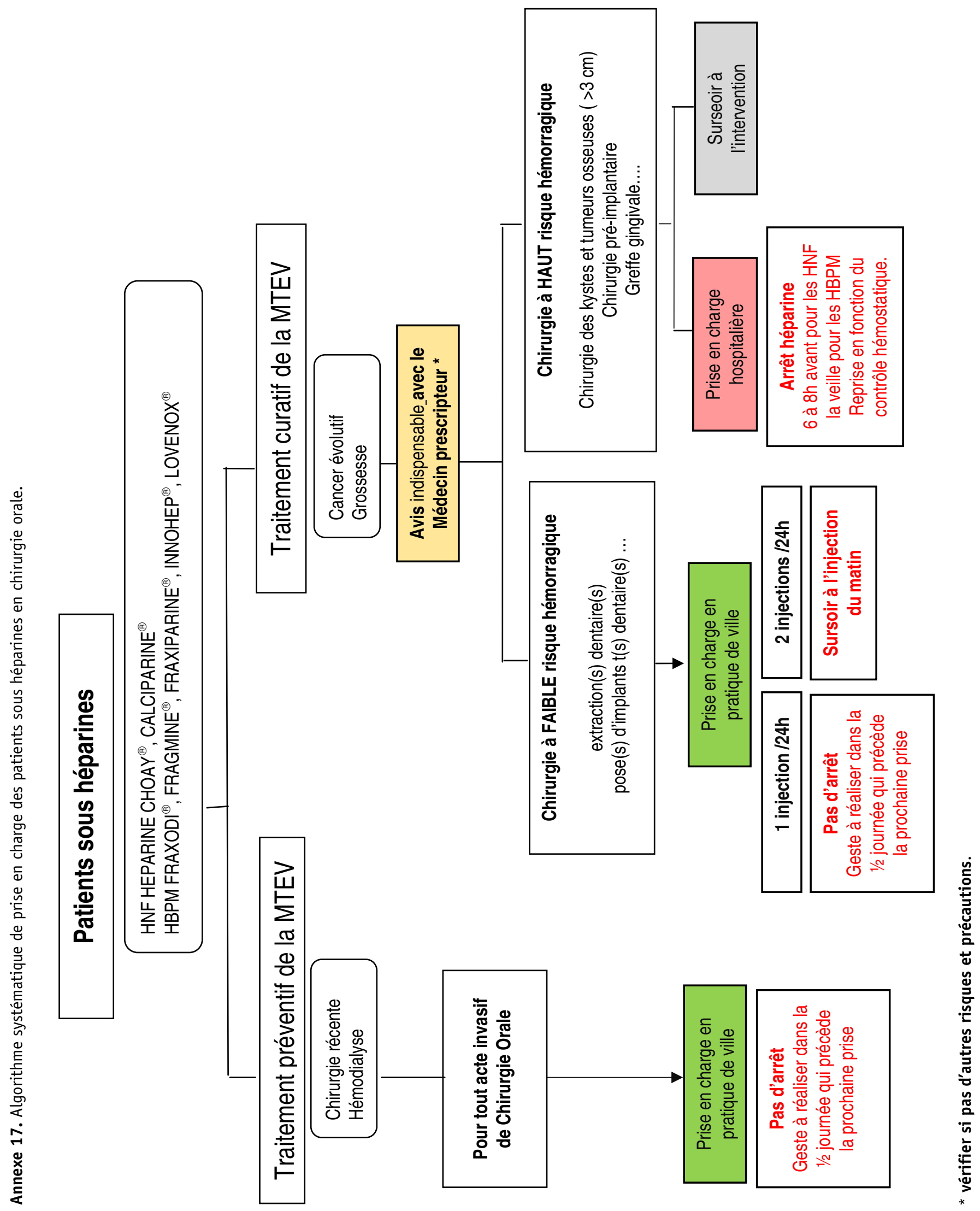




\section{REFERENCES}

1. Aframian DJ, Lalla RV, Peterson DE. Management of dental patients taking common hemostasis-altering medications. Oral Surg Oral Med Oral Pathol Oral Radiol Endod. 2007;103 (suppl 1): S45.e1-11.

2. Agence française de sécurité sanitaire des produits de santé (Afssaps). Commission de Transparence. Tissucol Kit à 500UI de thrombine/ml, poudres et solvants pour colle intralésionnelle 0,5 ml-1 ml-2 ml-5 ml. Avis du 16 juin 1999.

3. Agence française de sécurité sanitaire des produits de santé (Afssaps).Mise au point. Sur le bon usage des médicaments antivitamine K (AVK). Actualisation. Avril 2009.

4. Agence française de sécurité sanitaire des produits de santé (Afssaps). Prévention et traitement de la maladie thromboembolique veineuse. Recommandations de bonne pratique. Décembre 2009.

5. Agence française de sécurité sanitaire des produits de santé (Afssaps). Prescription des antibiotiques en pratique buccodentaire. Recommandations. Juillet 2011.

6. Agence française de sécurité sanitaire des produits de santé (Afssaps). Modification des recommandations sur la surveillance plaquettaire d'un traitement par Héparine de Bas Poids Moléculaire. Octobre 2011.

7. Agence française de sécurité sanitaire des produits de santé (Afssaps). Point d'information: Les nouveaux anticoagulants oraux (dabigatran et rivaroxaban) dans la fibrillation auriculaire : Ce qu'il faut savoir. Avril 2012.

8. Agence nationale de sécurité du médicament (Ansm) et Haute Autorité de Santé (HAS). Bon usage des antiplaquettaires. Juin 2012.

9. Agence nationale de sécurité du médicament et des produits de santé (Ansm). Les anticoagulants en France : état des lieux et surveillance. Juillet 2012.

10. Agence nationale de sécurité du médicament et des produits de santé (Ansm). Les anticoagulants en France en 2014 : état des lieux, synthèse et surveillance. Avril 2014.

11. Agence nationale de sécurité du médicament et des produits de santé (Ansm). Plan d'actions de l'ANSM sur les anticoagulants oraux directs en 2013-2014. Juillet 2014.

12. Ageno W, Gallus AS, Wittkowsky A, Crowther M, Hylek EM, Palareti G; American College of Chest Physicians. Oral anticoagulant therapy: antithrombotic therapy and prevention of thrombosis, 9th Ed: American College of Chest Physicians Evidence-Based Clinical Practice Guidelines. Chest 2012;141(2 Suppl):e44S-88S.

13. Airoldi F, Colombo A, Morici N. Incidence and predictors of drugeluting stent thrombosis during and after discontinuation of thienopyridine treatment. Circulation 2007;116:745-54.

14. Akopov SE, Suzuki S, Fredieu A, Kidwell CS, Saver SL, Cohen SN. Withdrawal of warfarin prior to surgical procedure: time to follow the guidelines? Cerebrovasc Dis 2005;19:337-42.

15. Albaladejo P. Synthèse et perspectives (Rivaroxaban). Ann Fr Anesth Rea 2008;27 Suppl 3:S28-31.

16. Albaladejo P, Godier A, Samama CM. Gestion périopératoire des nouveaux antithrombotiques. Le congrès. Médecins, Les essentiels 2012 Sfar. Page consulté le 29/08/2014 http:// sofia.medicalistes.org/spip/IMG/pdf/

Gestion_perioperatoire_des_nouveaux_antithrombotiques.pdf

17. Al-Belasy FA, Amer MZ.hemostatic effect of n-Butyl-2 cyanoacrylate(Histoacryl) glue in warfarin treated patients undergoing oral surgery. J Oral Maxillofac Surg 2003;61:1405-9.

18. Alcok RF, Reddel CJ, Pennings GJ, Hillis GS, Curnow JL, Brieger $\mathrm{DB}$. The rebound phenomenon after aspirin cessation: the biochemical evidence. Int J Cardiol 2014;174:376-8.

19. Al-Mubarak S, Rass MA, Alsuwyed A, Alabdulaaly A, Ciancio S. Thromboembolic risk and bleeding in patiens maintening or stopping oral anticoagulant therapy during dental extraction. $J$ Thromb Haemost 2006;4:689-91.

20. Al-Mubarak S, Al-Ali N, Abou-Rass M, Al-Sohail A, Robert A, AlZoman K, Al-Suwyed A, Ciancio S. Evaluation of dental extractions, suturing and INR on postoperative bleeding of patients maintained on oral anticoagulant therapy. $\mathrm{Br}$ Dent $\mathrm{J}$. 2007;203:E15; discussion 410-1.

21. Antithrombotic Trialists' Collaboration. Collaborative metaanalysis of randomised trials of antiplatelet therapyfor prevention of death, myocardial infarction, and stroke in high risk patients. Br Med J 2002;324(7329):71-86.

22. Antithrombotic Trialists Collaboration (ATT). Aspirin in the primary and secondary prevention of vascular disease: collaborative meta-analysis of individual participant data from randomised trials. Lancet 2009;373:1849-60.

23. António N, Castro G, Ramos D, Machado A, Gonçalves L, Macedo $T$, Providência LA. The debate concerning oral anticoagulation: whether to suspend oral anticoagulants during dental treatment. Rev Port Cardiol. 2008;27:531-44.

24. Ardekian L, Gaspar R, Peled M, Brener B, Laufer D. Does low-dose aspirin therapy complicate oral surgical procedures ? J Am Dent Assoc 2000;131:331-35.

25. Armstrong MJ, Schneck MJ, Biller J. Discontinuation of perioperative antiplatelet and anticoagulant therapy in stroke patients. Neurol Clin. 2006;24:607-30.

26. Aubertin MA. The patient taking antiplatelets drugs: a review with dental management considerations. General Dentistry 2008:363-69.

27. Autorité de la concurrence. Décision n¹0-D16 du 17 mai 2010 relatives à des pratiques mises en œuvre par la société SanofiAventis France. 120 p. www.autorité de la concurrence.fr/pdf/ avis/13d11.pdf (page consultée le 04.01.2014).

28. Bacci C, Maglione M, Favero L, Perini A, Di Lenarda R, Berengo M, Zanon E. Management of dental extraction in patients undergoing anticoagulant treatment. Results from a large, multicentre, prospective, case-control study. Thromb Haemost. 2010;104:972-5.

29. Bacci C, Berengo M, Favero L, Zanon E. Safety of dental implant surgery in patients undergoing anticoagulation therapy: a prospective case-control study. Clin Oral Implants Res. 2011;22:151-6.

30. Bachman DS. Discontinuing chronic aspirin therapy: another risk factor for stroke? Ann Neurol 2002;51:137-8. 
31. Bacourt F, Foster D, Mignon E. Athérosclérose oblitérante des membres inférieurs. Encycl Med Chir (Elsevier Masson, Paris) Angiologie, 19, 2010.

32. Baglin T, Keeling D, Kitchen S. Effects on routine coagulation screens and assessment of anticoagulant intensity in patients taking oral dabigatran or rivaroxaban: guidance from the British Committee for Standards in Haematology. Br J Haematology 2012;159:427-9.

33. Bajkin BV, Popovic SL, Selakovic SD. Randomized, prospective trial comparing bridging therapy using low-molecular-weight heparin with maintenance of oral anticoagulation during extraction of teeth. J Oral Maxillofac Surg. 2009;67:990-5.

34. Bajkin BV, Todorovic LM. Safety of local anaesthesia in dental patients taking oral anticoagulants: is it still controversial ? $\mathrm{Br}$ J Oral Maxillofac Surg. 2012a;50:65-8.

35. Bajkin BV, Bajkin IA, Petrovic BB. The effects of combined oral anticoagulant-aspirin therapy in patients undergoing tooth extractions: a prospective study. J Am Dent Assoc. 2012b;143:7716.

36. Bajkin BV, Urosevic IM, Stankov KM, Petrovic BB, Bajkin IA. Dental extractions and risk of bleding in patients taking single and dual antiplatelet treatment. Br J Oral Maxillofac Surg 2015, 53:29-43.

37. Balevi B. Should warfarin be discontinued before a dental extraction? A decision-tree analysis. Oral Surg Oral Med Oral Pathol Oral Radiol Endod. 2010;110:691-7.

38. Bandrowsky T, Vorono A, Borris TJ, Marcantoni HW. Amoxicillinrelated postextraction bleeding in an anticoagulated patient with tranexamic acid rinses. Oral Surg Oral Med Oral Pathol Oral Radiol Endod 1996;82:610-2.

39. Beirne OR. Evidence to continue oral anticogulant therapy for ambulatory oral surgery. J Oral Maxillofacial Surg 2005;63:540-5.

40. Biondi-Zoccai GG, Lotrionte M, Agostoni P et al., “A systematic review and meta-analysis on the hazards of discontinuing or not adhering to aspirin among 50279 patients at risk for coronary artery disease," European Heart Journal 2006, 27:2667-74.

41. Blinder D, Manor Y, Martinowitz U, Taicher S, Hashomer T. Dental extractions in patients maintained on continued oral anticoagulant: Comparison of local hemostatic modalities. Oral Surg Oral Med Oral Pathol Oral Radiol Endod 1999;88:137-40.

42. Blinder D, Manor Y, Martinowitz U, Taicher S. Dental extractions in patients maintened on oral anticoagulant therapy: comparison of INR value with occurrence of postoperative bleeding. Int J Oral Maxillofac Surg 2001;30:518-21.

43. Bloomer CR. Excessive hemorrhage after dental extractions using Low-Molecular-Weight Heparin (Lovenox) anticoagulation therapy. J Oral Maxillofac Surg 2004;62:101-3.

44. Boisramé-Gastrin S, Abgrall JF, Guillodo MP, Tanne F, Monguillon $P$. Les examens biologiques en chirurgie orale. In: Editions CdP WKF, editor: Manuel de chirurgie orale technique de réalisation pratique, maîtrise et exercice raisonné au quotidien. collection JPI0;2012:536p.

45. Bodner L, Weinstein JM, Baumgarten AK. Efficacy of fibrin sealant in patients on various levels of oral anticogaulant undergoing oral surgery. Oral Surg Oral Med Oral Pathol Oral Radiol Endod $1998 ; 86: 421-4$.
46. Borea G, Montebugnoli L, Capuzzi P, Magelli C. Tranexamic acid as mouthwash in anticoagulant-treated patients undergoing oral surgery. An alternative method to discontinuing anticoagulant therapy. Oral Surg Oral Med Oral Pathol 1993;75:29-31.

47. Breik 0, Cheng A, Sambrook PJ, Goss AN. Protocol in managing oral surgical patients taking dabigatran. Aust Dent J 2014;59: 296-301.

48. Brennan MT, Wynn RL, Miller CS. Aspirin and bleeding in dentistry: an update and recommandations. Oral Surg Oral Med Oral Pathol Oral Radiol Endod 2007;104:316-23.

49. Brennan MT, Hong C, Furney SL, Fox PC, Lockhart PB. Utility of an international normalized ratio testing device in a hospitalbased dental practice. J Am Dent Assoc. 2008;139:697-703.

50. Brennan MT, Valerin MA, Noll JL, Napenas JJ, Kent ML, Fox PC, Sasser HC, Lockhart PB. Aspirin use and post-operative bleeding from dental extractions. J Dent Res 2008;87:740-4.

51. Broekema F, van Minnen B, Jansma J, Bos RR. Risk of bleeding after dentoalveolar surgey in patients taking anticoagulants. $B r$ J Oral Maxillofac Surg 2014;52:e15-9.

52. Brooks AS. Delayed complications of tooth extraction in patients taking warfarin, antibiotics, and other medications. J Oral Maxillofac Surg 2011;69:977-9.

53. Bruggenkate CM, Krekeler G, Kraaijenhagen HA, Foitzik C, Nat $P$, Oosterbeek HS. Hemmorrhage of the floor of the mouth resulting from lingual peroration during implant placement: a clinical report. Int J Oral Maxillofac Implants 1993;8:329-34.

54. Cañigral A, Silvestre FJ, Cañigral G, Alós M, Garcia-Herraiz A, Plaza A. Evaluation of bleeding risk and measurement methods in dental patients. Med Oral Patol Oral Cir Bucal. 2010;15:e863-8.

55. Cannon PD, Dharmar VT. Minor oral surgical procedures in patients on oral anticoagulants-a controlled study. Aust Dent J 2003;48: 115-8.

56. Cardona-Tortajada F, Saint-Gómez E, Figuerido-Garmendia J, de Robles-Adsuar AL, Morte-Casabó A, Giner-Munoz F, etal. Dental extractions in patients on antiplatelet therapy. A study conducted by the Oral Health Departement of the Navarre Health Service (Spain). Med Oral Patol Oral Cir Bucal 2009a;14:e588-92.

57. Carter G, Goss A, Lloyd J, Tocchetti R. Tranexamic acid mouthwash versus autologous fibrin glue in patients taking warfarin undergoing dental extractions: a randomized prospective clinical study. J Oral Maxillofac Surg 2003a;61:1432-5.

58. Carter G, Goss A. Tranexamic acid mouthwash. A prospective randomized study of a 2-day regimen vs 5 -day regimen to prevent postoperative bleeding in anticoagulated patients requiring dental extractions. In J Oral Maxillofac Surg 2003b;32:504-7.

59. Casais P, Sanchez Luceros A, Meschengieser S, Fondevila C, Santarelli MT, Lazzari MA. Bleeding risk factors in chronic oral anticoagulation with acenocoumarol. Am J Hematol 2000;63: 192-6.

60. Chassot PG, Delabays A, Spahn DR, Perioperative antiplatelet therapy: the case for continuing therapy in patients at risk of myocardial infarction," British Journal of Anaesthesia 2007;99: 316-28.

61. Chee YL, Crawford JC, Watson HG, Greaves M. Guidelines on the assessment of bleeding risk prior to surgery or invasive 
procedures. British committee for standards in haematology. British Journal of Haematology 2008;140:496-504.

62. Chen ZM, Jiang LX, Chen YP, Xie JX, Pan HC, Peto R, Collins R, Liu LS, COMMIT collaborative group. Addition of clopidogrel to aspirin in 45,852 patients with acute myocardial infaction : randomised placebo-controlled trial. Lancet 2005;366(9497): 1607-21.

63. Cocero N, Mozzati M, Ambrogio M, Bisi M, Morello M, Bergamasco L. Bleeding rate during surgery of oral anticoagulant therapy patients with associated systemic pathologic entities: a prospective study of more than 500 extractions. J Oral Maxillofac Surg 2014;72:858-67.

64. Collet JP, Himbert F, Steg PG. Myocardial infaction after aspirin cessation in stable coronary artery disease patients. Int $\mathrm{J}$ Cardiol 2000;76:257-8.

65. Collet JP, Montalescot G, Blanchet B, Tanguy ML, Golmard JL, Choussat R, Beygui F, Payot L, Vignolles N, Metzger JP, Thomas D. Impact of prior use or recent withdrawing of oral antiplatelet agents on acute coronary syndromes. Circulation 2004;110:2361-7.

66. Conan M, Massot M, Clipet F, Alno N, Lejeune S, De Mello G. Étude du rapport coût/sécurite lors de la prise en charge des patients sous antivitamines $\mathrm{K}$ en chirurgie buccale. Med Buccale Chir Buccale 2009;15:17-30.

67. Connolly SJ, Ezekowitz MD, Yusuf S, Eikelboom J et al. Dabigatran versus warfarin in patient with atrial fibrillation. $N$ Engl J Med 2009;361:1139-51.

68. Connolly SJ, Eikelboom J, Joyner C et al. Apixaban in patients with atrial fibrillation. N Engl J Med 2011;364:806-17.

69. Cooke GE, Liu-Sratton Y, Kerketich AK et al. Effect of platelet antigen polymorphism on plaqtelet inhibition by aspirin, clopidogrel, or their combination. J Am Coll Cardiol 2006;47:541-6.

70. Cosmi B, Alatri A, Cattaneo M, Gresele P, Marrieta M and Italian Society for Haesmostasis and Thrombosis. Assessment of the risk of bleeding in patients undergoing surgery or invasive procedures. Guidelines. Thromb Res 2009;124(5):e6-e12.

71. Crowther MA, Warkentin TE. Bleeding risk and the management of bleeding complications in patients undergoing anticoagulant therapy: focus on new anticoagulant agents. Blood 2008; 111:4871-9.

72. Czembireck C, Poeschl WP, Eder-Czembirek C, Fischer MB, Perisanidis C, Jesch Ph, Schicho K, Dong A, Seemann R. Causes and timing of delayed bleeding after oral surgery. Clin Oral Invest 2014;18:1655-61.

73. Daïmellah F, Issad MS, Boukaïs H, Zerrouki W, Berkane M, Lehachi S, Bennoui Z, Khellil S, Hannoun D, Abrouk S. Avulsions dentaires chez les patients cardiaques traités par anticoagulants : résultats d'un essai thérapeutique acénocoumarol versus héparine calcique. Med Buccale Chir Buccale 2009;15:63-74.

74. Daïmellah F, Issad MS, Lehachi S, Bennoui Z, Khelil S, Boukaïs $H$, Zerrouki W, Berkane M, Hannoun D, Abrouk S. Facteurs de risque hémorragique chez les patients sous antivitamine $\mathrm{K}$ en chirurgie buccale. Med Buccale Chir Buccale 2010;16:209-15.

75. Darriba MA, Mendonça-Caridad JJ. Profuse bleeding and lifethreatening airway obstruction after placement of mandibular dental implants. J Oral Maxillofac Surg 1997;55:1328-30.

76. Dasgupta A, Steinhubl SR, Bhatt DL, Berger PB, Shao M, Mak KH, Fox KA, Montalescot G, Weber MA, Haffner SM, Dimas AP, Steg PG,
Topol EJ;CHARISMA Investigators. Clinical outcomes of patients with diabetic nephropathy randomized to clopidogrel plus aspirin versus aspirin alone (a post hoc analysis of the clopidogrel for high atherothrombotic risk and ischemic stabilization, management, and avoidance trial. Am J Cardiol. 2009;103:1359-63.

77. Davis C, Robertson C, Shivakumar S, Lee Min. Implications of dabigatran, a direct thrombin inhibitor, for oral surgery practice. J Can Dent Asooc 2013;7979:d74:1-7.

78. Davi G, Patrono C. Platelet activation and atherothrombosis. $N$ Engl J Med 2007;357:2482-94.

79. Davydov L, Yermolnik M, Cuni LJ. Warfarin and amoxicillin/clavulanate drug interaction. Ann Pharmacother 2003;37:367-70.

80. Degirmenci SE, Steib A. Les anticoagulants dans la prevention de la thrombose veineuse. Rev Prat 2013;63:976-9.

81. Desmard M, Hellmann R, Plantefève G, Mentec H. Surdosage grave en antivitamine $\mathrm{K}$ secondaire à l'absorption de jus de pamplemouse. Ann Fr Anesth Reanima 2009;28:897-9.

82. Devani P, Lavery KM, Howell CJT. Dental extractions in patients on warfarin: is alteration of anticoagulant regime necessary ? $\mathrm{Br}$ J Oral Maxillofac Surg 1998;36:107-11.

83. Doonquah L, Mitchell AD. Oral surgery for patients on anticoagulant therapy: current thoughts on patient management. Dent Clin North Am 2012;56:25-41.

84. Douketis JD, Johnson JA, Turpie AG. Low-molecular - weight heparins bridging anticoagulation during interruption of warfarin assessment of a standardized periprocedural anticoagulation regimen. Arch Intern Med 2004;164:1319-26.

85. Douketis JD, Berger PB, Dunn AS, Jaffer AK, Spyropoulos AC, Becker RC, Ansell J; American College of Chest Physicians. The perioperative management of antithrombotic therapy: American College of Chest Physicians Evidence-Based Clinical Practice Guidelines (8th Edition). Chest. 2008;133:299S-339S.

86. Douketis JD, Berger PB, Dunn AS, Jaffer AK, Spyropoulos AC, Becker RC, Ansell J; American College of Chest Physicians. The perioperative management of antithrombotic therapy: American College of Chest Physicians Evidence-Based Clinical Practice Guidelines (9th Edition). Chest. 2012;141:e326S-e350S.

87. Douxfils J, Mullier F, Robert S, Chatelain C, Chatelain B, Dogné JM. Impact of dabigatran on a large panel of routine or specific coagulation assays. Thromb Haemost 2012;107:985-97.

88. Ducroq G, Steg PG. The role of new antiplatelet agents in the therapeutic stategy. Arch Cardiovascular Dis Suppl. 2012;4:195-9.

89. Dunn CJ, Goa KL. Tranexamic acid: a review of its use in surgery and other indications. Drugs 1999;57:1005-32.

90. Eerenberg ES, Kampuhuisen PW, Sijpkens MK, Meijers JC, Buller $H R$, Levi M. Reversal of rivaroxaban and dabigatran by prothrombin complex concentrate: a randomized, placebocontrolled, crossover study in healthy subjects. Circulation 2011; 124:1573-9.

91. Eikelboom JW, Hirsh J, Spencer FA, Baglin TP, Weitz JI. Antiplatelet drugs: antithrombotic therapy and prevention of thrombosis, $9^{\text {th }}$ ed: American College of Chest Physicians Evidence-Based Clinical Practice Guidelines. Chest 2012;141 (2 Suppl): e89S119 S.

92. EINSTEIN Investigators. Oral rivaroxaban for symptomatic venous thromboembolism. N Engl J Med 2010;363:2499-510. 
93. EINSTEIN-PE Investigators. Oral rivaroxaban for the treatment of symptomatic pulmonary embolism. N Engl J Med. 2012;366: 1287-97.

94. Eriksson BI, Dahl OE, Rosencher N, Kurth AA, van Dijk CN, Frostick SP, Chritiansen AV, Hantel S, Hettiarachchi R, Schnee J, Buller HR. Oral Dabigatran etexilate versus enoxaparin for the prevention of venous thromboembolism after total knee replacement: the RE-MODEL randomized trial. J Thromb Haemost 2007;5 (11):2178-85.

95. Eriksson BI, Dahl OE, Rosencher N, Kurth AA, van Dijk CN, Frostick SP, Prins MH, Hettiarachchi R, Hantel S, Schnee J, Buller HR. Dabigatran etexilate versus enoxaparin for prevention of venous thromboembolism after total hip replacement: a randomised, double-blind, non-inferiority trial (RE-NOVATE I). Lancet 2007;370:949-56.

96. Eriksson BI, Borris LC, Friedman RJ, Haas S, Huisman MV, Kakkar AK, Bandel TJ, Beckmann H, Muehlofer E, Misselwitz F, Geertz W. Rivaroxaban versus enoxaparin for thrombophylaxis after hip arthroplasty (RECORD 1). N Engl J Med. 2008;358:2765-75.

97. Eriksson BI, Smith $H$, Yasothan U, Kirkpatrick P. Dabigatran etexilate. Nat Rev Drug Discov 2008;7:557-8.

98. Eriksson BI, Dahl OE, Huo MH, Kurth AA, Hantel S, Hermansson K, Schnee JM, Friedman RJ.Oral dabigatran versus enoxaparin for thromboprophylaxis after primary total hip arthroplasty (RENOVATE $\left.\mathrm{II}^{*}\right)$. A randomised, double-blind, non-inferiority trial. Thromb Haemost 2011;105(4):721-9.

99. Evans IL, Sayers MS, Gibbons AJ, Price G, Snooks H, Sugar AW. Can warfarin be continued during dental extraction ? Results of a randomized controlled trial. Br J Oral Maxillofac 2002;40: 248-52.

100. Ezekowitz MD, Connolly S, Parekh A, Reilly PA, Varrone J, Wang S, Oldgren J, Themeles E, Wallentin L, Yusuf S. Rationale and design of RE-LY: randomized evaluation of long-term anticoagulant therapy, warfarin, compared with dabigatran. $A m$ Heart J 2009;157:805-10.

101. Fakhri HR, Janket SJ, Jackson EA, Baird AE, Dinnocenzo R, Meurman $\mathrm{JH}$. Tutorial in oral antithrombotic therapy: biology and dental implications. Med Oral Patol Oral Cir Bucal 2013;18: e461-72.

102. Ferrari E, Benhamou M, Cerboni P, Baudouy M. Coronary syndromes following aspirin withdrawal. Chest 2003;124:148S.

103. Ferrari E, Benhamou M, Cerboni P, Baudouy M. Coronary syndromes following aspirin withdrawal. A special risk for late stent thrombosis. J Am Coll Cardiol 2005;45:456-9.

104. Ferrieri GB, Castiglioni S, Carmagnola D, Cargnel M, Strohmenger $\mathrm{L}$, Abati S. Oral surgery in patients on anticoagulant treatment without therapy interruption. J Oral Maxillofac Surg 2007;65: 1149-54.

105. Firriolo FJ, Hupp WS. Beyond warfarin: the new generation of oral anticoagulants and their implications for the management of dental patients. Oral Surg Oral Med Oral Pathol Oral Radiol 2012;113:431-41.

106. Fugh-Berman A. herb-drug interactions. Lancet 2000;355:134-8.

107. Funayama M, Kumagai T, Saito K, Watanabe T. Asphyxial death caused by postextraction hematoma. Am J Forensic Med Pathol 1994;15:87-90.
108. Gagneja M, Gagneja P, Steelman R, Shaughnessy R, Johannes PW. Oral surgery in a child with a prosthetic aortic valve and pulmonary artery stent at risk for thromboembolism. J Clin Pediatr Dent 2008;32:151-3.

109. Gangloff P. Prise en charge des patients traités par agents antiplaquettaires et antivitamines k. In: Editions CdP WKF, editor: Manuel de chirurgie orale: Technique de réalisation pratique, maîtrise et exercice raisonné au quotidien. Collection JPI0 ;2012:536 p.

110. Garcia DA, Regan S, Henault LE, Upadahyay A, Baker J, Othman M, Hylek EM. Risk of thromboembolism with short-term interruption of warfarin therapy. Arch Intern Med 2008;168: 63-69.

111. Garcia DA, Baglin TP, Weitz JI, Samama MM; American College of Chest Physicians. Parenteral anticoagulants: Antithrombotic Therapy and Prevention of Thrombosis, 9th ed: American College of Chest Physicians Evidence-Based Clinical Practice Guidelines. Chest. 2012;141(2 Suppl):e24S-43S.

112. Garcia Rodriguez LA, Cea-Soriano L, Martin-Merino E. Discontinuation of low-dose aspirin and risk of myocardila infarction: cas-control study in UK primary care. $\mathrm{Br}$ Med $\mathrm{J}$ 2011;343:d4094.

113. Garnier J, Truchot F, Quero J, Meziere X, Clipet F, Alno N, Frachon X, Delanoue 0, Bader G, Lejeune S, Limbour P, De Mello G. 218 tooth extraction in patients tacking platelet aggregation inhibitors. Rev Stomatol Chir Maxillofac 2007;108:407-10.

114. Gaspar R, Brenner B, Ardekian L, Peled M, Laufer D. Use of tranexamic acid mouthwash to prevent postoperative bleeding in oral surgery patients on oral antcoagulant medication. Quintessence Int 1997;28:375-9.

115. Gaudy JF, Ankri A, Tager F, El Haddioui A, Bravetti P, Lafont A, Gogly B. Anticoagulants et extractions dentaires. Arch Mal Coeur Vaiss. 2005;98:859-66.

116. Gerstein NS, Schulman PM, Gerstein WH, Petersen TR, Tawil I. Should more patients continue aspirin therapy perioperatively? Clinical impact of aspirin withdrawal syndrome. Ann Surg 2012;255(5):811-9.

117. Givol N, Chaushu G, Halamish-Shani T, Taicher S. Emergency tracheostomy following life-threatening hemorrhage in the floor of the mouth during immediate implant placement in the mandibular canine region. J Periodontol 2000;71:1893-5.

118. Gersel-Pedersen N. Fibrinolytic activity of blood an d saliva before and after oral surgery. Int J de Oral Surg 1981;10 (Suppl1):114-21.

119. Graff J, von Hentig N , Misselwitz F, Kubitza D, Becka M, Breddin HK, Harder S. Effects of the oral, direct factor Xa inhibitor rivaroxaban on platelet-induced thrombin generation and prothrombinase activity. J Clin Pharmacol 2007;47:1398-407.

120. Granger CB, Alexander JH, McMurray JJ, Lopes RD, Hylek EM, Hanna M, Al-Khalidi HR, Ansell J, Atar D, Avezum A, Bahit MC, Diaz R, Easton JD, Ezekowitz JA, Flaker G, Garcia D, Geraldes M, Gersh BJ, Golitsyn S, Goto S, Hermosillo AG, Hohnloser SH, Horowitz J, Mohan P, Jansky P, Lewis BS, Lopez-Sendon JL, Pais P, Parkhomenko A, Verheugt FW, Zhu J, Wallentin L, ARISTOTLE Committees and Investigators. Apixaban versus warfarin in patients with atrial fibrillation. N Engl J Med 2011;365:981-92. 
121. Grines CL, Bonow R0, Casey Jr DE, Gardner TJ, Lockhart PB, Moliterno DJ, O'Gara P, Whitlow P. Prevention of premature discontinuation of dual antiplatelet therapy in patients with coronary artery stents: a science advisory from American Heart Association, American College of Cardiology, Society for Cardiovascular Angiography and Interventions, American College of Surgeons, and American Dental Association, with representation from the American College of Physicians. J Am Dent Assoc 2007;138:652-655.

122. Groupe d'Étude sur l'Hemostase et de la Thrombose (GEHT). Prise en charge des surdosages, des situations à risque hémorragique et des accidents hémorragiques chez les patients traités par antivitamines $\mathrm{K}$ en ville et en milieu hospitalier. Recommandations pour la pratique clinique. Avril 2008.

123. Groupe d'Étude sur l'Hémostase et de la Thrombose (GEHT). Rivaroxaban et test de biologie medicale. Octobre 2012.

124. Groupe d'Étude sur l'Hémostase et de la Thrombose (GEHT). Héparine, dérivés hépariniques et antagonistes de la vitamine K. Maniement, surveillance biologique, gestions des complications. Décembre 2012.

125. Guyat GH, AkL EA, Crowther M, Gutterman DD, Schünemann HJ. Antithrombotic therapy and prevention of thrombosis $\left(9^{\text {th }}\right.$ ed).American College of Chest Physicians. Evidence-Based Clinical Practice Guidelines. Chest 2012;141(Suppl):7S-47S.

126. Halfpenny W, Fraser JS, Adlam DM. Comparison of 2 hemostatic agents for the prevention of postextraction hemorrhage in patients on anticoagulants. Oral Surg Oral Med Oral Pathol Oral Radiol Endod 2001;92:257-9.

127. Hall R, Mazer CD. Antiplatelet drugs: a review of their pharmacology and management in the perioperative period. Anesth Analg 2011;112:292-318.

128. Harder S. Renal profiles of anticoagulants. J Clin Pharmacol 2012;52:964-75.

129. Hart RG, Benavente 0, McBride R et al. Antithrombotic therapy to prevent stroke in patients with atrial fibrillation : a metaanlysis. Ann Intern Med 1999;131:492-501.

130. Haute Autorité de Santé (HAS). Commission de Transparence. Mini-sintrom $1 \mathrm{mg}$, comprimé et Sintrom $4 \mathrm{mg}$, comprimé quadrisécable. Avis du 2 novembre 2005.

131. Haute Autorité de Santé (HAS). Commission de Transparence. Feiba $500 \mathrm{UI} / 20 \mathrm{ml}$, poudre et solvant pour solution injectable. Avis du 30 novembre 2005.

132. Haute Autorité de Santé (HAS). Commission de Transparence. Persantine $75 \mathrm{mg}$, comprimé enrobé. Avis du 10 mai 2006.

133. Haute Autorité de Santé (HAS). Commission de Transparence. Cardiosolupsan, poudre effervescente pour solution buvable. Avis du 19 juillet 2006.

134. Haute Autorité de Santé (HAS). Commission de Transparence. Fraxodi et Fraxiparine, solution injectable SC en seringue préremplie avec système de sécurité. Avis du 18 octobre 2006.

135. Haute Autorité de Santé (HAS). Commission de Transparence. Plavix 75 mg, comprimé pelliculé. Avis du 06 juin 2007.

136. Haute Autorité de Santé (HAS). Commission de Transparence. Calciparine sous-cutanée, solution injectable. Avis du 23 janvier 2008.
137. Haute Autorité de Santé (HAS). Commission de Transparence. Lovenox, solution injectable (sous-cutanée) en seingue préremplie. Avis du 2 avril 2008.

138. Haute Autorité de Santé (HAS). Commission de Transparence. Héparine Choay $25000 \mathrm{UI} / 5 \mathrm{ml}$, solution injectable (IV). Avis du 3 septembre 2008 .

139. Haute Autorité de Santé (HAS). Commission de Transparence.Efient 10mg, comprimé pelliculé. Avis du 22 juillet 2009.

140. Haute Autorité de Santé (HAS). Commission de Transparence.Innohep, solution injectable (sous-cutanée) en seringue pré-remplie. Avis du 16 décembre 2009.

141. Haute Autorité de Santé (HAS). Commission de Transparence. Duoplavin $75 \mathrm{mg} / 75 \mathrm{mg}$, comprimé pelliculé. Avis du 21 juillet 2010.

142. Haute Autorité de Santé (HAS). Commission de Transparence. Asasantine LP $200 \mathrm{mg} / 25 \mathrm{mg}$, gélule à libération prolongée. Avis du 15 décembre 2010.

143. Haute Autorité de Santé (HAS). Commission de Transparence. Pravadual, comprimé. Avis du 2 février 2011.

144. Haute Autorité de Santé (HAS). Commission de Transparence. Previscan $20 \mathrm{mg}$, comprimé quadrisécable. Avis du 20 juillet 2011.

145. Haute Autorité de Santé (HAS). Commission de Transparence. Kardégic $75 \mathrm{mg}, 160 \mathrm{mg}, 300 \mathrm{mg}$, poudre pour solution buvable en sachet dose. Avis du 21 septembre 2011.

146. Haute Autorité de Santé (HAS). Commission de Transparence. Brilique $90 \mathrm{mg}$, comprimé pelliculé. Avis du 7 décembre 2011. septembre 2011.

147. Haute Autorité de Santé (HAS). Commission de Transparence. Coumadine $2 \mathrm{mg}, 5 \mathrm{mg}$, comprimé sécable. Avis du $1^{\mathrm{er}}$ fevrier 2012.

148. Haute Autorité de Santé (HAS). Commission de Transparence. Pradaxa $110 \mathrm{mg}, 150 \mathrm{mg}$, comprimé pelliculé. Avis du 14 mars 2012

149. Haute Autorité de Santé (HAS). Commission de Transparence. Xarelto $15 \mathrm{mg}, 20 \mathrm{mg}$, gélules. Avis du 29 février 2012.

150. Haute Autorité de Santé (HAS). Commission de Transparence.Ticlid 250mg, comprimé pélliculé. Avis du 11 avril 2012.

151. Haute Autorité de Santé (HAS). Commission de Transparence. Exacyl $1 \mathrm{~g} / 10 \mathrm{ml}$, solution buvable. Avis du 12 juin 2013.

152. Haute Autorité de Santé (HAS). Commission de Transparence. Eliquis 2,5mg, $5 \mathrm{mg}$, comprimé pellicullé. Avis du 12 juin 2013.

153. Haute Autorité de Santé (HAS). Commission de Transparence. Fragmine, solution injectable, seringue préremplie. Avis du 22 janvier 2014.

154. Haute Autorité de Santé (HAS). Guide du médecin- Affection de longue durée- Maladie coronarienne. mars 2007.

155. Haute Autorité de Santé (HAS). Guide du médecin- Affection de longue durée- Accident vasculaire cérébral. mars 2007.

156. Haute Autorité de Santé (HAS). Guide du médecin- Affection de longue durée- Artériopathie oblitérante des membres inférieurs. mars 2007.

157. Haute Autorité de Santé (HAS). Guide du médecin- Affection de longue durée- Fibrillation auriculaire. juillet 2007.

158. Haute Autorité de Santé (HAS). Guide du médecin- Affection de longue durée- Cardiopathies valvulaires et congénitales graves chez l'adulte. juin 2008. 
159. Haute Autorité de Santé (HAS). Indicateurs de pratique clinique et indicateurs d'alerte et de maîtrise de la iatrogénie. Contrôle de l'INR si AVK et introduction d'un antibiotique ou d'un antifongique. Octobre 2012.

160. Haute Autorité de Santé (HAS). Rapport d'évaluation technologique. Hémostatiques chirurgicaux. Juin 2011.

161. Haute Autorité de Santé (HAS). Rapport d'évaluation technologique. Biologie des anomalies de l'hémostase. Tome 1 : temps de saignement (Epreuve de Duke et tests d'Ivy). Juillet 2011.

162. Haute Autorités de Santé (HAS). Recommandations de bonne pratique. Antiagrégants plaquettaires: prise en compte des risques thrombotique et hémorragique en cas de geste endoscopique chez le coronarien. Méthode Recommandation par consensus formalisé. Juin 2012.

163. Haute Autorité de Santé (HAS). Recommandation de bonne pratique. Antiagrégants plaquettaires: prise en compte des risques thrombotique et hémorragique pour les gestes percutanés chez le coronarien. Méthode Recommandation par consensus formalisé. Novembre 2013.

164. Healey JS, Eikelboom J, Douketis J, Wallentin L, Oldgren J, Yang S, Themeles E, Heidbuchel H, Avezum A, Reilly P, Connolly SJ, Yusuf S, Ezekowitz M; RE-LY Investigators. Periprocedural bleeding and thromboembolic events with dabigatran compared with warfarin: results from the Randomized Evaluation of LongTerm Anticoagulation Therapy (RELY) randomized trial. Circulation 2012;126:343-8.

165. Ho $M$, Peterson $E$, Wang $L$ et al. Incidence of death and acute muocardial infarctus associated with stopping clopidogrel after acute coronary syndrome. J Am Med Assoc 2008;299:532-9.

166. Ho M, Tsai T, Wang T et al. Adverse events after stopping clopidogrel in post-acute coronary syndrome patients. Insights from a large integrated healthcare delivery system. Cir Cardiovasc Qual Outcomes 2010;3:303-8.

167. Hong CH, Napeñas JJ, Brennan MT, Furney SL, Lockhart PB. Frequency of bleeding following invasive dental procedures in patients on low-molecular-weight heparin therapy. J Oral Maxillofac Surg 2010;68:975-9.

168. Hong C, Napeñas JJ, Brennan M, Furney S, Lockhart P. Risk of postoperative bledding after dental procedures in patients on warfarin: a retrospective study. Oral Surg Oral Med Oral Pathol Oral Radiol Endod 2012;114:464-8.

169. Hong YH, Mun SK. A case of massive maxillary sinus bleeding after dental implant. Int J Oral Maxillofac Surg 2011;40:758-60.

170. Hughes GJ, Patel PN, Saxena N. Acetaminophen on International Normalized Ratio in patients receiving warfarin therapy. Pharmacotherapy 2011;31:591-7.

171. Hylek EM, Heiman H, Skates SJ, Sheehan MA, Singer DE. Acetaminophen and other risk factors for excessive warfarin anticoagulation. JAMA. 1998;279:657-62.

172. Iung B. Extractions dentaires sous anticoagulants: améliorer la prise en charge par une approche multidisciplinaire. Arch Mal Coeur Vaiss. 2005;98:857-8.

173. Iwabuchi H, Imai Y, Asanami S, Shirakawa M, Yamane GY, Ogiuchi H, Kurashina K, Miyata M, Nakao H, Imai H. Evaluation of postextaction bleeding incidence to compare patients receiving and not receiving warfarin therapy: a cross-sectional, multicenter, observational study. BMJ Open 2014;4:e005777. Doi10.1136/ bmjopen-2014-005777.

174. James S, Akerblom A, Cannon CP, Emanuelsson H, Husted S, Katus H, Skene A, Steg PG, Storey RF, Harrington R, Becker R, Wallentin L. Comparison of ticagrelor, the first reversible oral P2Y12 receptor antagonist, with clopidogrel in patients with acute coronary syndromes: rationale, design, and baseline characteristics of the PLATelet inhibition and patient Outcomes (PLATO) trial. Am Heart J 2009;157:599-605.

175. Jensen SS, Eriksen J, Schiodt M. Severe bleeding after sinus floor elevation using the transcrestal technique: a case report. Eur J Oral Implantol 2012;5:287-291.

176. Johnson-Leong C, Rada RE. The use of low-molecular-weight heparins in out patient oral surgery for patients receiving anticoagulation therapy. J Am Dent Assoc 2002;133:1083-7.

177. Journal Officiel de la République Française. Décision du 11 février 2013 de l'Union nationale des caisses d'assurance maladie relative à la liste des actes et prestations pris en charge par l'assurance maladie «sous-chapitre 5-02 _Hémostase et coagulation ».

178. Kakkar AK, Brenner B, Dahl OE, Eriksson BI, Mouret P, Muntz J, Soglian AG, Akos F, Misselwitz F, Hass S. Extensed duration rivaroxaban versus short term enoxaparin for the prevention of venous thromboembolism after total hip arthroplasty: a doublebind randomized controlled trial (RECORD 2). The Lancet 2008;372 (9632):31-39.

179. Kalpidis CD, Konstantinidis AB. Critical hemorrhage in the floor of the mouth during implant placement in the first mandibular premolar position: a case report. Implant Dent 2005;14:177-84.

180. Karsh ED, Erdogan 0̈, Esen E, Acartürk E. Comparison of the effects of warfarin and heparin on bleeding caused by dental extraction: a clinical study. J Oral Maxillofac Surg 2011; 69:2500-7.

181. Keiani Motlagh K, Loeb I, Legrand W, Daelemans P, van Reck J. Prévention des saignements postopératoires chez des patients sous anticoagulants oraux. Effets de l'acide tranexamique. Rev Stomatol Chir Maxillofac 2003;104:77-9.

182. Kinnby B, Lindberg P, Lecander I, Matsson L. Localization of plasminogen activators and plasminogen-activator inhibitors in human gingival tissues demonstrated by immunohistochemistry and in situ hybridization. Arche Oral Biol 1999;44:1027-1034.

183. Kosyfaki P, Att W, Strub JR. The dental patient on oral anticoagulant medication: a literature review. J Oral Rehabil. 2011;38:615-33.

184. Kovacs MJ, Kearon C, Rodger M, Anderson DR, Turpie AG, Bates MD, Desjardins L, Doukertis MD, Kahn SR, Solymoss S, Wells PS. Single -arm of study of briding therapy with low-molecularweight heparin for patients at risk of arterial embolism who require temporary interruption of warfarin. Circulation $2004 ; 110$ : 1658-63.

185. Krishnan B, Sheny NA, Alexander M. Exodontia and antiplatelet therapy. J Oral Maxillofac Surg 2008;66:2063-6.

186. Laboda G. Life-threatening hemorrhage after placement of an endosseous implant: report of case. J Am Dent Assoc 1990;121: 599-600. 
187. Lane MA, Devine ST, Mc Donald JR. High-risk antimicrobial prescriptions among ambulatory patients on warfarin.J Clin Pharmacy Therap 2011: doi:10.1111/j.1365-2710.2011.01270.x

188. Larsen TR, Gelaye A, Durando C. Acute warfarin toxicity: an unanticipated consequence of amoxicillin/clavulanate administration. Am J Case Rep 2014;15:45-8.

189. Lassen MR, Ageno W, Lars C, Borris MD, Jay R, Liberman MB, Rosencher N, Tiemo J, Bandel MD, Missewitz F, Turpie AG. Rivaroxaban versus enoxaparin for thrombophylaxis after total knee arthroplasty (RECORD 3). N Eng J Med 2008;358:2776-86.

190. Lega JC, Bertoletti L, Durupt S, Epinat M, Mismetti P, Da Costa A. Nouveaux anticoagulants oraux dans la fibrillation atriale non valvulaire. La Presse Médicale, 2013 ;42(9):1225-31.

191. Le Heuzey JY, Marijon E, Lepillier A, Fiorina L, Charlemagne A, Lavergne $T$, Pornin M. La fibrillation atriale: données démographique. www.réalitéscardiologiques.com/wp-contenu/ uploads/2010/03/10.pdf. (page consulté le 05.01.2014).

192. Leonardi-Bee J, Bath PM, Bousser MG, Davalos A, Diener HC, Guiraud-Chaumeil B, Sivenius J, Yatsu F, Dewey ME. Dipyridamole for preventing recurrent ischemic stroke and other vascular events: A meta-analysis of individual patient data from randomized controlled trials. Stroke. 2005;36:162-8.

193. Lesca C, Boumendjel S, Boumendjel M, Hefied M, Ben Ismail S, Bonnefous D. Local haemostasis with an adhesive cyano-coated membrane following tooth extraction in patients under anticoagulant or antiplatelet therapy. Rev Stomatol Chir Maxillofac 2012;113:143-7.

194. Levi MM, Eerenberg E, Lowenberg E, Kamphuisen PW. Bleeding in patients using new anticoagulants or antiplatelet agents: risk factors and management. Neth J Med 2010;68:68-76.

195. Lev-Ran 0, Kramer A, Gurevitch J. Low-molecular weight heparin for prosthetic heart valves. Ann Thorac Surg 2000;69:264.

196. Lewis BS, Mehta SR, Fox KA, Halon DA, Zhao F, Peters RJ, Keltai $M$, Budaj A, Yusuf S, CURE trial investigators. Benefit of clopidogrel according to timing of percutaneous coronary intervention in patients with acute coronary syndromes: further results from the Clopidogrel in Unstable angina to prevent Recurrent Events study. Am Heart J 2005;150 (6):1177-84.

197. Lillis T, Ziakas A, Koskinas K, Tsirlis A, Giannoglou G. Safety of dental extractions during uninterrupted single or dual antiplatelet treatment. Am J Cardiol 2011;108:964-7.

198. Lind SE. The bleeding time does not predict surgical bleeding. Blood 1991;77(12):2547-52.

199. Lockhart PB, Gibson J, Pond SH, Leicht J. Dental management considerations for the patient with an acquired coagulopathy. Part 1: coagulopathies from systemic disease. Br Dent $\mathrm{J}$ 2003;195:439-45.

200. Lopes RD, Horowitz JD, Garcia DA, Crowther MA, Hylek EM. Warfarin and acetaminophen interaction: a summary of the evidence and biologic plausibility. Blood 2011;118:62769- 73.

201. Lordkipanidze M, DiodatiJG, Pharand Ch. Possibility of a rebound phenomenon following antiplatelet therapy withdrawal : a look at the clinical and pharmacological evidence. Pharmacology and Therapeutics 2009;123:178-85.
202. Lowry JC. Thromboembolic disease and thromboprophylaxis in oral and maxillofacial surgery. Experience and practice. $\mathrm{Br} \mathrm{J}$ Oral Maxillofac Surg 1995;33:101-6.

203. Mac DonaldsTM, Wei L. Effect of ibuprofen on cardioprotective effect of aspirin. Lancet 2003;361:573-4.

204. Madan GA, Madan SG, Madan G, Madan AD. Minor oral surgery without stopping daily low-dose aspirin therapy: a study of 51 patients. J Oral Maxillofac Surg 2005;65:1262-5.

205. Madrid C, Sanz M. What influence do anticoagulants have on oral implant therapy ? A systematic review. Clin Oral Implants Res 2009;20:96-106.

206. Mahe I, Bertrand N, Drouet L, Bal Di Sollier Cl, Simoneau G, Mazoyer E, Caulin Ch, Bergmann JF. Intercation between paracetamol and warfarin in patients: a double-blind, placebocontrolled, randomized study. Haematologica 2006;91:1621-7.

207. Makris M, Van Veen JJ, Tait CR, Mumford AD, Laffan M, Guideline on the management of bleeding in patients on antithrombotic agents. Br J Haematology 2012;160:35-46.

208. Malden NJ, Santini A, Mather CI, Gardner A. Minor oral surgery and interference with anticoagulation in patients taking warfarin: a retrospective study. Br J Oral Maxillofac Surg 2007;45: 645-7.

209. Martinowitz U, Mazr AL, Taicher S, Varon D, Gitel SN, Ramot B, Rakocz M,. Dental extraction for patients on oral anticoagulant therapy. Oral Surg Oral Med Oral Pathol 1990;70:274-7.

210. Mas JL. Atrial fibrillation: thromboembolic complications. Arch Cardio Dis Suppl 2013;5:125-31.

211. Masson ME, Triplet RG, Alfonso WF. Life-threatening hemorrhage from placement of a dental implant. J Oral Maxillofac Surg 1990;48:201-4.

212. Medeiros FB, de Andrade AC, Angelis GA, Conrado VC, Timerman L, Farsky P, Dib LL. Bleeding evaluation during single tooth extraction in patients with coronary artery disease and acetylsalicylic acid therapy suspension : a prospective, doubleblinded, and randomized study. J Oral Maxillofac Surg 2011;69:2949-55.

213. Mehta SR, Yusuf S; Clopidogrel in Unstable angina to prevent Recurrent Events Study Investigators. The Clopidogrel in Unstable angina to prevent Recurrent Events (CURE) trial programme; rationale, design and baseline characteristics including a metaanalysis of the effects of thienopyridines in vascular disease. Eur Heart J. 2000;21:2033-41.

214. Michel P. L'arrêt de l'aspirine augmente le risqué de récidive d'accident vasculaire cerebral. 30th International Stroke Conference. Stroke 2005;36:416 (abstract).

215. Michelson AD. New P2Y12 antagonists. Curr Opin Hematol 2009;16:371-7.

216. Minassian C, D'Aiuto F, Hingorani AD, Smeeth L. Invasive dental treatment and risk for vascular events: a self-controlled cases series. Ann Intern Med 2010;153:499-506.

217. Mismetti P, Laporte S. Rivaroxaban: clinical pharmacology. Ann Fr Anesth Rea 2008;27:S16-S21.

218. Moghadam HG, Caminiti MF.Life-threatening hemorrhage after extraction third molars: case report and management protocol. J Can Dent Assoc 2002;68:670-4. 
219. Montalescot G, Wiviott SD, Braunwald E, Murphy SA, Gibson CM, McCabe CH, Antman EM; TRITON-TIMI 38 investigators. Prasugrel compared with clopidogrel in patients undergoing percutaneous coronary intervention for ST-elevation myocardial infarction (TRITON-TIMI 38): double-blind, randomised controlled trial. Lancet 2009;373:723-31.

220. Mordenfeld A, Andersson L, Bergström B. Hemorrhage in the floor of the mouth during implant placement in the edentulous mandible: a case report. Int J Oral Maxillofac Implants 1997;12: 558-561.

221. Moreira P, Filho PM, Silva EA, Weksler C, Drable SG, Tura BR, Fonseca Mda G, Cunha AB, Fischer RG. Effect of periodontal treatment on oral anticoagulation in patients with heart disease. Rev Port Cardiol. 2007;26:977-89.

222. Moreno R, Fernandez C, Hernandez R, Alfonso F, Angiolillo DJ, Sabate M, Escaned J, Banuelos C, Fernandez-Ortiz A, Macaya C. Drug-eluting stent thrombosis: results from a pooled analysis including 10 randomized studies. J Am Coll Cardiol. 2005;45:9549.

223. Morimoto $\mathrm{Y}$, Niwa $\mathrm{H}$, Minematsu K. Hemostatic management of tooth extractions in patients on oral antithrombotic therapy. $J$ Oral Maxillofac Surg. 2008a;66:51-7.

224. Morimoto $\mathrm{Y}$, Niwa $\mathrm{H}$, Hanatani A, Nakatani T. Hemostatic management during oral surgery in patients with a leftventricular assist system undergoing high-level anticoagulant therapy: efficacy of low molecular weight heparin. J Oral Maxillofac Surg. 2008b;66:568-71.

225. Morimoto $Y$, Niwa H, Minematsu K. Risk factors affecting postoperative hemorrhage after tooth extraction in patients receiving oral antithrombotic therapy. J Oral Maxillofac Surg 2011;69:1550-6.

226. Morimoto $Y$, Niwa $H$, Minematsu K. Risk factors affecting hemorrhage after tooth extraction in patients undergoing continuous infusion with unfractionated heparin. J Oral Maxillofac Surg. 2012;70:521-6.

227. Napenas JJ, Hong CH, Brennan MT, Furney SL, Fox PC, Lockhart $\mathrm{PB}$. The frequency of bleeding complications after invasive dental treatment in patients receiving single and dual antiplatelet therapy. J Am Dent Ass 2009;140:690-5.

228. Napenas JJ, Oost FC, deGroot A, Loven B, Hong CH, Brennan MT, Lockhart PB, van Dierman DE. Review of postoperative bleeding risk in dental patients on antiplatelet therapy. Oral Surg Oral Med Oral Pathol Oral Radiol 2013;115:491-9.

229. Nematullah A, Alabousi A, Blanas N, Douketis JD, Sutherland SE. Dental surgery for patients on anticoagulant therapy with warfarin: a systematic review and meta-analysis. J Can Dent Assoc. 2009;75:41-41i.

230. Niamtu J. Near-fatal airway obstruction after routine implant placement. Oral Surg Oral Med Oral Pathol Oral Radiol Endod 2001;92:597-600.

231. Nizamaldin Y, Abi Najm S, El Hage M, Samson J. Hémostase locale en chirurgie orale. 1ère partie : physiologie de l'hémostase. Med Buccale Chir Buccale 2012a;18:119-127.

232. Nizamaldin $Y$, Samson J. Hémostase locale en Chirurgie orale. 2ème partie : efficacité de la colle de fibrine. Med Buccale Chir Buccale 2012b;18:193-210.
233. Oger E. Incidence of venous thromboembolism: A communitybased study in western France. Epi-getbp study group. Groupe d'étude de la thrombose de bretagne occidentale. Thromb Haemost. 2000;83:657-60.

234. Olmos-Carrasco 0, Pastor-Ramos V, Espinilla-Blaco R, OrtizZarate A, Garcia-Avilla I, Rodriguez-Alonso E, Herrero-Sanjuan R, Ruiz-Garcia MM, Gallego-Beuter P, Sanchez-Salgado MP, TeranAugustin AI, Fernandez-Behar M, Pena-Sainz I. Hemorrhagic complications of dental extractions in 181 patients undergoing double antiplaqtelet therapy. J Oral Maxillofac Surg $2015 ; 73$ : 203-10.

235. Panula K, Oikarinen K. Severe hemorrhage after implant surgery. Oral Surg Oral Med Oral Pathol Oral Radiol Endod 1999;87 (1):2.

236. Patatanian E, Fugate SE. Hemostatic mouthwashes in anticoagulated patients undergoing dental extraction. Ann Pharmacother. 2006;40:2205-10.

237. Patridge CG, Campbell JH, Alvarado F. The effect of plateletaltering medications on bleeding from minor oral surgery procedures. J Oral Maxillofac Surg 2008;66:93-7.

238. Patel MR, Mahaffey KW, Garg J, Pan G, Singer DE, Hacke W, Breithardt G, Halperin JL, Hankey GJ, Piccini JP, Becker RC, Nessel CC, Paolini JF, Berkowitz SD, Fox KA, Califf RM, (ROCKET AF Investigators). Rivaroxaban versus warfarin in nonvalvular atrial fibrillation. N Engl J Med 2011;365:883-91.

239. Park MW, Her SH, Kwon JB, Lee JB, Choi MS, Cho JS et al. Safety of dental extractions in coronary drug-eluting stenting patients without stopping multiple antiplatelet agents. Clin Cardiol 2012;35:225-30.

240. Payne DA, Hayes PD, Jones CI, Belham P, Naylor AR, Goodall AH. Combined therapy with clopidogrel and aspirin significantly increases the bleeding time through a synergistic antiplatelet action. J Vasc Surg 2002;35:1204-9.

241. Penning-van Best FJ, van Meegen E, Rosendaal FR, Stricker BH. Drug interactions as a cause of overanticoagulation on phenprocoumon or acenocoumarol predominantly concern antibacterial drugs. Clin Pharmacol Ther 2001;69:451-7.

242. Pettinger TK, Owens CT. Use of low-molecular-weight heparin during dental extractions in a medicaid population. J Manag Care Pharm. 2007;13:53-8.

243. Pernod G, Albaladejo P, Godier A, Samama CM, Susen S, Gruel Y, Blais N, Fontana P, Cohen A, Llau JV, Schved JF, de Maiste E, Samama MM, Sié $P$. Prise en charge des complications hémorragiques graves et de la chirurgie en urgence chez des patients recevant un anticoagulat oral anti-IIa ou anti-Xa direct. Propositions du Groupe d'Intérêt en Hémostase Périopératoire (GIHP)-mars 2013. Ann Fr Anesth Reanim 2013; http:// dx.doi.org/10.1016/j.annfar.2013.04.016

244. Perry DJ, Noakes TJ, Helliwell PS; British Dental Society. Guidelines for the management of patients on oral anticoagulants requiring dental surgery. Br Dent J. 2007;203:389-93.

245. Persac S, Boland FX, Lavis JF, Tardif A. Avulsions dentaires et anticoagulants. Rev Stomatol Chir Maxillofac. 2007;108:189-92.

246. Pisters R, Lane DA, Nieuwlaat R, de Vos CB, Crijns HJ, Lip GY. A novel user-friendly score (has-bled) to assess 1-year risk of major bleeding in patients with atrial fibrillation: The euro heart survey. Chest 2010;138:1093-100. 
247. Pototski M, Amenábar JM. Dental management of patients receiving anticoagulation or antiplatelet treatment. J Oral Sci. 2007;49:253-8.

248. Rai R, Mohan B, Pratap Singh V, Namita, Wander GS. The risk of bleeding during dental extractions in patients receiving antiplatelet therapy. Indian J Dental Sciences 2013;4 (5):016018.

249. Ramström G, Sindet-Pedersen S, Hall G, Blombäck M, Alander U. Prevention of Postsurgical bleeding in oral surgery using tranexamic acid without dose modification of oral anticoagulants. J Oral Maxillofac Surg 1993;51:1211-6

250. Rodgers RP, Levin J. A critical reappraisal of bleeding time. Semin Thromb Hemost 1990;16:1-20.

251. Rossi ML, Zavalloni D, Gasparini GL, Presbitero P. Very late multivessel thrombosis of bare stents with concomitant patent drug-eluting stent after withdrawal of aspirin. Int $\mathrm{J}$ Cardiol 2008;131:e7-9.

252. Rossini R, Capodanno D. Prevalence predictors and long-term prognosis premature discontinuation of oral antiplatelet therapy after eluting stent implantation. Am J Cardiol 2011;107:186-94.

253. Sabatine MS, Cannon CP, Gibson CM, Lopez-Sendon JL, Montalescot G, Theroux P. Addition of clopidogrel to aspirin and fibrinolytic therapy for myocardial infarction with ST-segment elevation (CLARITY study). N Engl J Med 2005;352:1179-89.

254. Sacco R, Sacco M, Carpenedo M, Mannucci PM. Oral surgery in patients on oral anticoagulant therapy: a randomized comparison of different intensity targets. Oral Surg Oral Med Oral Pathol Oral Radiol Endod. 2007;104:e18-21.

255. Salam S, Yusuf H, Milosevic A. Bleeding after dental extractions in patients taking warfarin. Br J Oral Maxillofac Surg. 2007;45: 463-6.

256. Samama MM, Conard J, Lillo-Le-Louët A. Accidents hémorragiques des nouveaux anticoagulants oraux et examens de coagulation. J Mal Vasc 2013;38:259-270.

257. Sambu N, Warner T, Curzen N. Clopidogrel withdrawal is there a « rebound » phenomenon ? Thromb Haemost 2011;105:211-20.

258. Schulman S, Kearon C, Kakkar AK, Mismetti P, Schellong S, Eriksson H, Baanstra D, Schnee J, Goldhaber SZ. Dabigatran versus warfarin in the treatment of acute venous thromboembolism (RECOVER study). New Eng J Med 2009;361:2342-52.

259. Sié P, Samama CM, Godier A, Rosencher N, Steib A, Llau JV, van der Linden P, Pernod G, Lecompte T, Gouin-Thibault I, Albaladejo $P$. Chirurgies et actes invasifs chez les patients traités au long cours par un anticoagulant oral anti-Iia ou anti-Xa direct. Propositions du groupe d'intérêt en hémostase périopératoire (GIHP) et du groupe d'études sur l'hémostase et la thrombose (GEHT). Ann Fr Anesth Reanim 2011a;30:645-50.

260. Sié P, Samama CM, Godier A, Rosencher N, Steib A, Llau JV, Van der Linden P, Pernod G, Lecompte T, Gouin-Thibault I, Albaladejo P; Working Group on Perioperative Haemostasis; French Study Group on Thrombosis and Haemostasis. Surgery and invasive procedures in patients on long-term treatment with direct oral anticoagulants: thrombin or factor-Xa inhibitors. Recommendations of the Working Group on Perioperative Haemostasis and the French Study Group on Thrombosis and Haemostasis. Arch Cardiovasc Dis 2011b;104:669-76.
261. Siguret V, Esquirol C, debray M, Gouin I, Andreux JP, Pautas E. Surdosages en antivitamine $\mathrm{K}$ dans une population de patients hospitalisés âgés de plus de 70 ans. Enquête prospective sur un an. Press Med 2003;32:972-7.

262. Simonet V, Cambus JP, Léger P, Boneu B. Antivitamines K : utilisation pratique. Encyclopédie Médico-chirurgicale, 2003 13022-D-50.

263. Sindet-Pedersen S, Ramström G, Bernil S, Blombäck M. Hemostatic effect of tranexamic acid mouthwash in anticoagulant treated patients undergoing oral surgery. N Engl J Med $1989 ; 320$ : 840-3.

264. Société française d'Anesthésie et de Réanimation (Sfar). Prévention de la maladie thromboembolique veineuse périopératoire et obstétricale. Recommandations pour la pratique clinique [en ligne]. 2005: http://www.sfar.org/t/spip.php,article270

265. Société française d'Anesthésie et de Réanimation (Sfar). Examens pré interventionnels systématiques. 13 janvier 2012.

266. Société Francophone de Médecine Buccale et Chirurcie buccale (SFMbCb). Recommandations pour la prise en charge des patients sous agents antiplaquettaires en odontostomatologie. Med Buccale Chir Buccale 2005;11;2:55-76

267. Société francophone de Médecine Buccale et Chirurcie buccale (SFMbCb). Recommandations pour la prise en charge des patients sous traitement anti-vitamines $\mathrm{k}$ en chirurgie bucco-dentaire. Med Buccale Chir Buccale2006;188-212.

268. Soto J, Sacristan JA, Fernandez-Viadero C, Verduga R. Probable acenocoumarol-amoxycillin interaction. Acta Haematol 1993;90: 195-7.

269. Souto JC, Oliver A, Zuazu-Jausoro I, Vives A, Fontcuberta J. oral surgery in anticoagulated patients without reducing the dose of oral anticoagulant: a prospective randomized study. J Oral Maxillofac Surg 1996;54:27-32.

270. Spertus JA, KettelkampR, Vance C, Decker C, Jones PG, Rumsfeld JS, Messenger JC, Khanal S, Peterson ED, Bach RG, Krumholz HM, Cohen DJ. Prevalence, predictors, and outcomes of premature discontinuation of thienopyridine therapy after drug-eluting stent placement. Results from the PREMIER registry. Circulation 2006;113;2803-9.

271. Spolarich AE, Andrews L. An examination of the bleeding complications associated with herbal supplements, antiplatelet and anticoagulant medications. J Dent Hyg. 2007;81:67.

272. Spyropoulos AC, Turpie AGG, Spandorfer J. Clinical outcomes with unfractionated heparin or low-molecular -weight heparin as bridging therapy in patients on long-term oral anticoagulants: The REGIMEN Registry. J Thromb Haemost 2006;4:1246.

273. Spyropoulos AG, Turpie AGG, Dunn AS. Perioperative bridging therapy with unfractionned heparin or low-molecular weight heparin as bridging therapy in patients with mechanical prosthectic heart valves on long term oral anticoagulants (from the REGIMEN registry). Am J Cardiol 2008;102:883

274. Spyropoulos AG., To bridge or not to bridge. That is the question. The argument for bridging therapy in patients on oral anticoagulants requiring temporary interruption for elective procedure. J Thromb Thrombolysis 2010;29:192.

275. Sugidachi A, Ogawa T, Kurihara A, Hagihara K, Jakubowski JA, Hashimoto M, Niitsu Y, Asai F. The greater in vivo antiplatelet 
effects of prasugrel as compared to clopidogrel reflect more efficient generation of its active metabolite with similar antiplatelet activity to that of clopidogrel's active metabolite. $J$ Thromb Haemost 2007;5:1545-51.

276. Todd W, Roman A. Outpatient use of Low-Molecular Weight Heparin in an anticoagulated patient requiring oral surgery: case report. J Oral Maxillofac Surg 2011;59:1090-2.

277. Václavík J, Táborský M. Antiplatelet therapy in the perioperative period. Eur J Intern Med 2011;22:26-31.

278. Van Diermen D, Aartman IHA, Baart JA, Hoogstraten J, van der Waal I. Dental management of patients using antithrombotic drugs critical appraisal of existing guidelines. Oral Surg Oral Med Oral Pathol Oral Radiol Endod 2009;107:616-24.

279. Van Ryn J, Strangler J, Haertter S, Liesenfeld KH, Wienen W, Feuring $M$, Clemens A. Dabigatran etexilate--a novel, reversible, oral direct thrombin inhibitor: interpretation of coagulation assays and reversal of anticoagulant activity. Thromb Haemost 2010;103:1116-27.

280. Verma G, Tiwari AK, Chopra S. Aspirin and exodontias: a comparative study of the bleeding complications with aspirin therapy. International Journal of Dental Science and Research 2013;1:50-3.

281. Verma G. Dental extraction can be performed safely in patients on aspirin therapy: a timely reminder. ISRN Dentistry 2014, Article ID463684,http//:dx.dor.0rg/10.1155/2014/463684.

282. Vidal. Le dictionnaire. 2014;90e edition, Issy-les-Moulineau, France. www.vidal.fr

283. Wahl MJ. Dental surgery and anticoagulated patients. Arch Intern Med 1998;158:1610-6.

284. Wallentin L, Becker RC, Budaj A, Cannon CP, Emanuelsson H, Held $C$, et al. Ticagrelor versus clopidogrel in patients with acute coronary syndromes. N Engl J Med 2009;361:1045-57.

285. Wittkowsky AK. Dietary supplements, herbs and oral anticoagulants: the nature of the evidence. J Thromb Thrombolysis 2008;25:72-77.

286. Wiviott SD, Braunwald E, McCabe CH, Montalescot G, Ruzyllo W, Gottlieb S, Neumann FJ, Ardissino D, De Servi S, Murphy SA,
Riesmeyer J, Weerakkody G, Gibson CM, Antman EM; TRITON-TIMI 38 Investigators. Prasugrel versus clopidogrel in patients with acute coronary syndromes. N Engl J Med 2007;357:2001-15.

287. Wong PC, Crain EJ, Xin B, Wexler RR, Lam PY, Pinto DJ, Luettgen JM, Knabb RM. Apixaban, an oral, direct and highly selective factor Xa inhibitor: in vitro, antithrombotic and antihemostatic studies. J Thromb Haemost 2008;6:820-9.

288. Wong PC, Pinto DJ, Zhang D. Preclinical discovery of apixaban, a direct and orally bioavailable factor Xa inhibitor. J Thromb Thrombolysis 2011;31:478-92.

289. Wynn RL. Bleeding risks for older patients taking warfarin and commonly prescribed antibiotics and antifungals simultaneously. Gen Dent. 2012;60:454-6.

290. Zanon E, Martinelli F, Bacci C, Cordioli G, Girolami A. Safety of dental extraction among consecutive patients on oral anticoagulant treatment managed using a specific dental management protocol. Blood coagulation \& fibrinolysis 2003;14: 27-30.

291. Zhang Q, Bal dit Sollier C, Simoneau G, Alvarez JC, Pruvot S, Aubourg R, Berge N, Bergmann JF, Mouly S, Mahé I. Ineraction between acetominophen and warfarin in adults receiving longterm oral anticoagulants: a randomized controlled trial. Eur J Clin Pharmacol 2011;67:309-14.

292. Zhang Q, Simoneau G, Verstuyft C, Drouet L, Bal dit Sollier C, Alvarez JC, Rizzo-Padoin N, Bergmann JF, Becquemont L, Mouly S. Amoxicillin/clavulanic acid- warfarin drug interaction : a randomized controlled trial. Br J Clin Pharmacol 2011;71:232-36.

293. Romond KK, Miller CS, Henry RG. Dental management considerations for a patient taking dabigatran etexilate: a case report. Oral Surg Oral Med Oral Pathol Oral Radiol 2013;116:e191e195.

294. Meyer G, Belmont L. Cancer and venous thromboembolism. Rev Mal Resp 2011;25:443-52.

295. Pollack CV, Reilly PA, Eikelboom J, Glund S, Verhamme P, Bernstein RA, Dubiel R, Huisman MV, Hylek EM, Kamphuisen PW, Kreuzer J, Levy JH, Sellke FW, Strangier J, Steiner T, Wang B, Kam CW, Weitz JI. Idarucizumab for dabigatran reversal. New Engl J Med 2015, Jun 22. 